\title{
Electronic Structure and Bonding in Iron(II) and Iron(I) Complexes Bearing Bisphosphine Ligands of Relevance to Iron-Catalyzed C-C Cross-Coupling
}

Jared L. Kneebone, Valerie E. Fleischauer, Stephanie L. Daifuku, Ari A. Shaps, Joseph M. Bailey, Theresa E. Iannuzzi and Michael L. Neidig*

Department of Chemistry, University of Rochester, Rochester, New York 14627, USA

E-mail: neidig@chem.rochester.edu 


\section{Table of Contents}

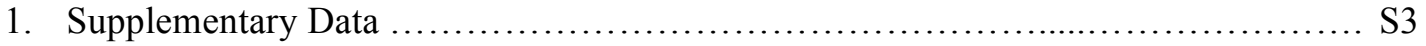

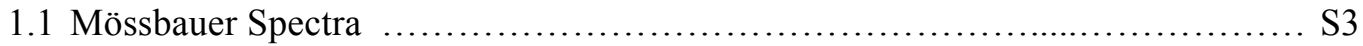

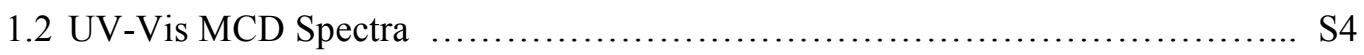

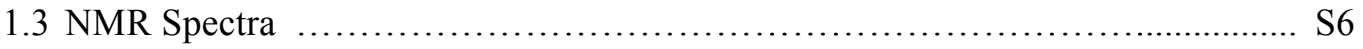

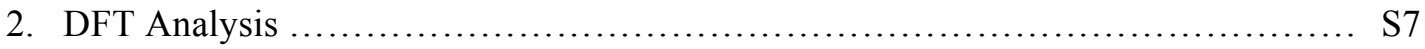

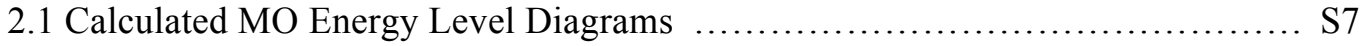

2.2 TD-DFT Assignments of CT Transitions for $\mathrm{FeCl}_{2}$ (bisphosphine) Complexes .... S12

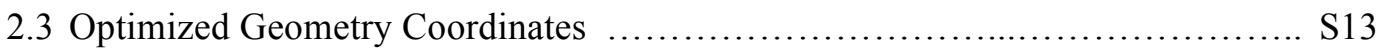

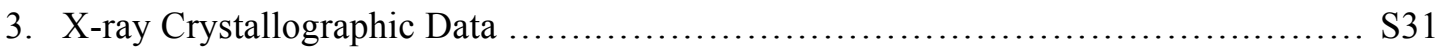

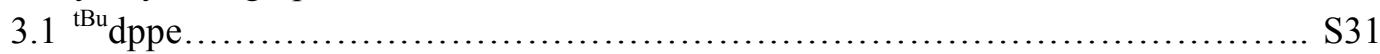

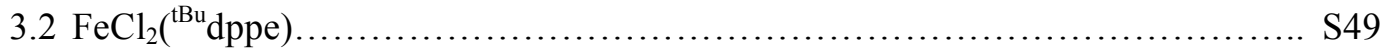

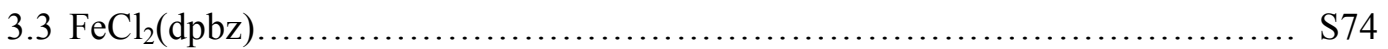

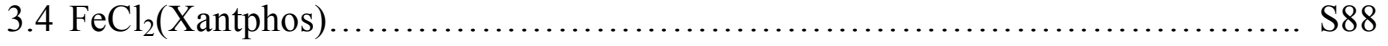

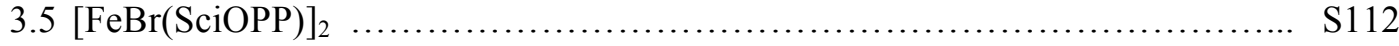




\section{Supplementary Data}

\subsection{Mössbauer Spectra}

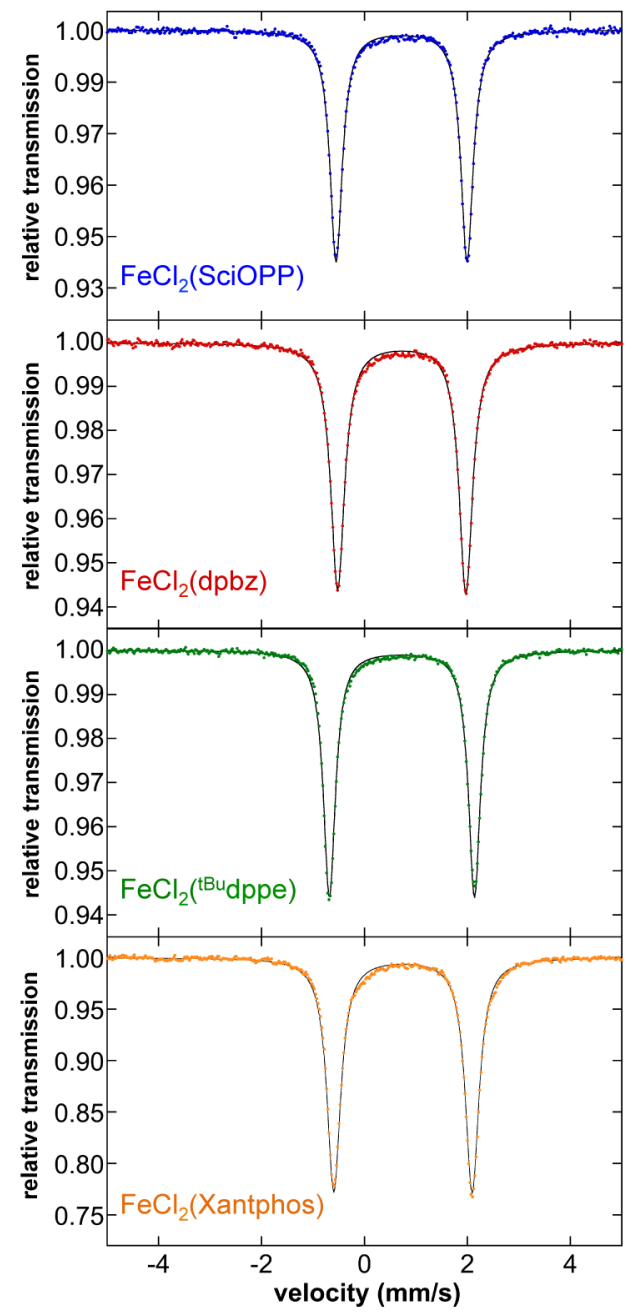

Figure S1. $80 \mathrm{~K}$, Solid-state ${ }^{57} \mathrm{Fe}$ Mössbauer spectra of $\mathrm{FeCl}_{2}$ (bisphosphine) complexes. The best-fit parameters for each complex are given in the manuscript in Table 1. 


\subsection{UV-Vis MCD Spectra}

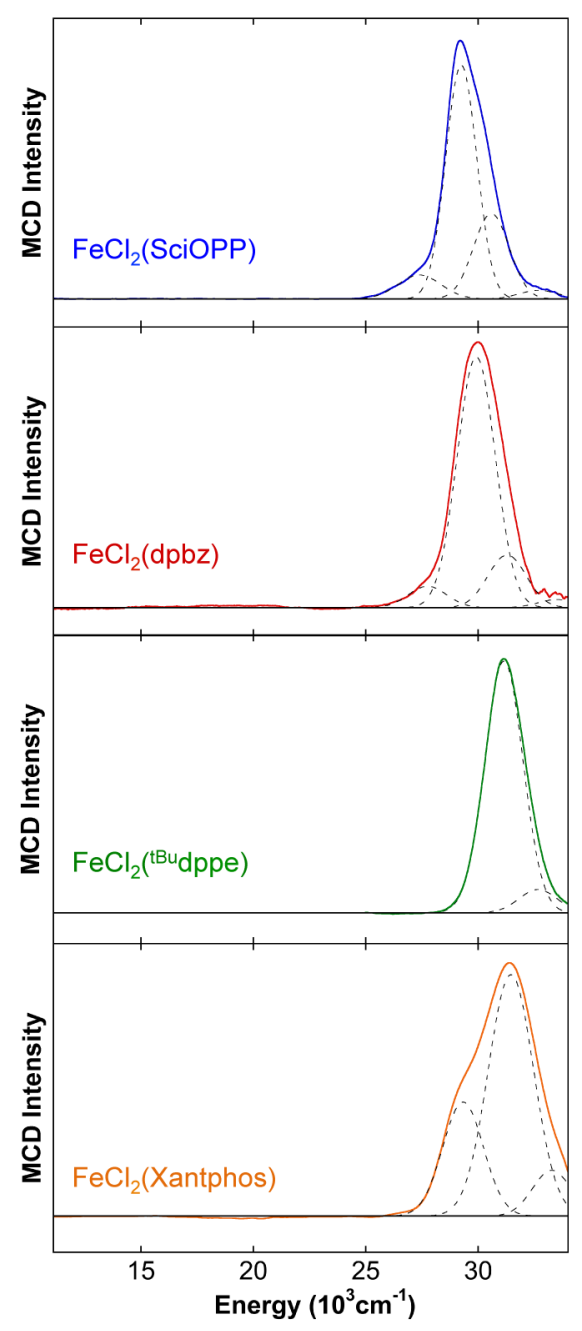

Figure S2. UV-Vis MCD spectra of $\mathrm{FeCl}_{2}$ (bisphosphine) complexes. Data (solid lines) and individual transition Gaussian fits (dashed lines) are given for each complex. The $\mathrm{FeCl}_{2}(\mathrm{SciOPP})$ spectrum was collected at $15 \mathrm{~K}$ and $7 \mathrm{~T}$ whereas all other spectra were collected at $5 \mathrm{~K}$ and $7 \mathrm{~T}$. All spectra were collected in $6: 1$, toluene- $\mathrm{d}_{8}$ : benzene- $\mathrm{d}_{6}$ except for $\mathrm{FeCl}_{2}$ (Xantphos) which was collected on a solid mull sample. 


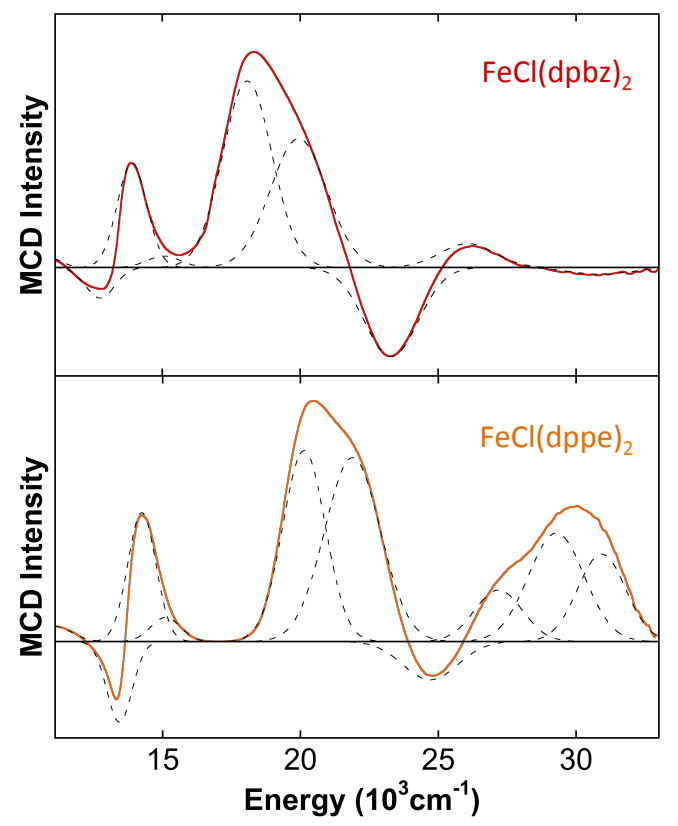

Figure S3. $5 \mathrm{~K}, 7 \mathrm{~T} \mathrm{UV}-\mathrm{V}$ is $\mathrm{MCD}$ spectra of $\mathrm{FeCl}(\mathrm{dpbz})_{2}$ and $\mathrm{FeCl}(\mathrm{dppe})_{2}$. Data (solid lines) and individual transition Gaussian fits (dashed lines) are given for each complex. Spectra were collected in 6:1, toluene- $\mathrm{d}_{8}:$ benzene- $\mathrm{d}_{6}$ 


\subsection{NMR Spectra}
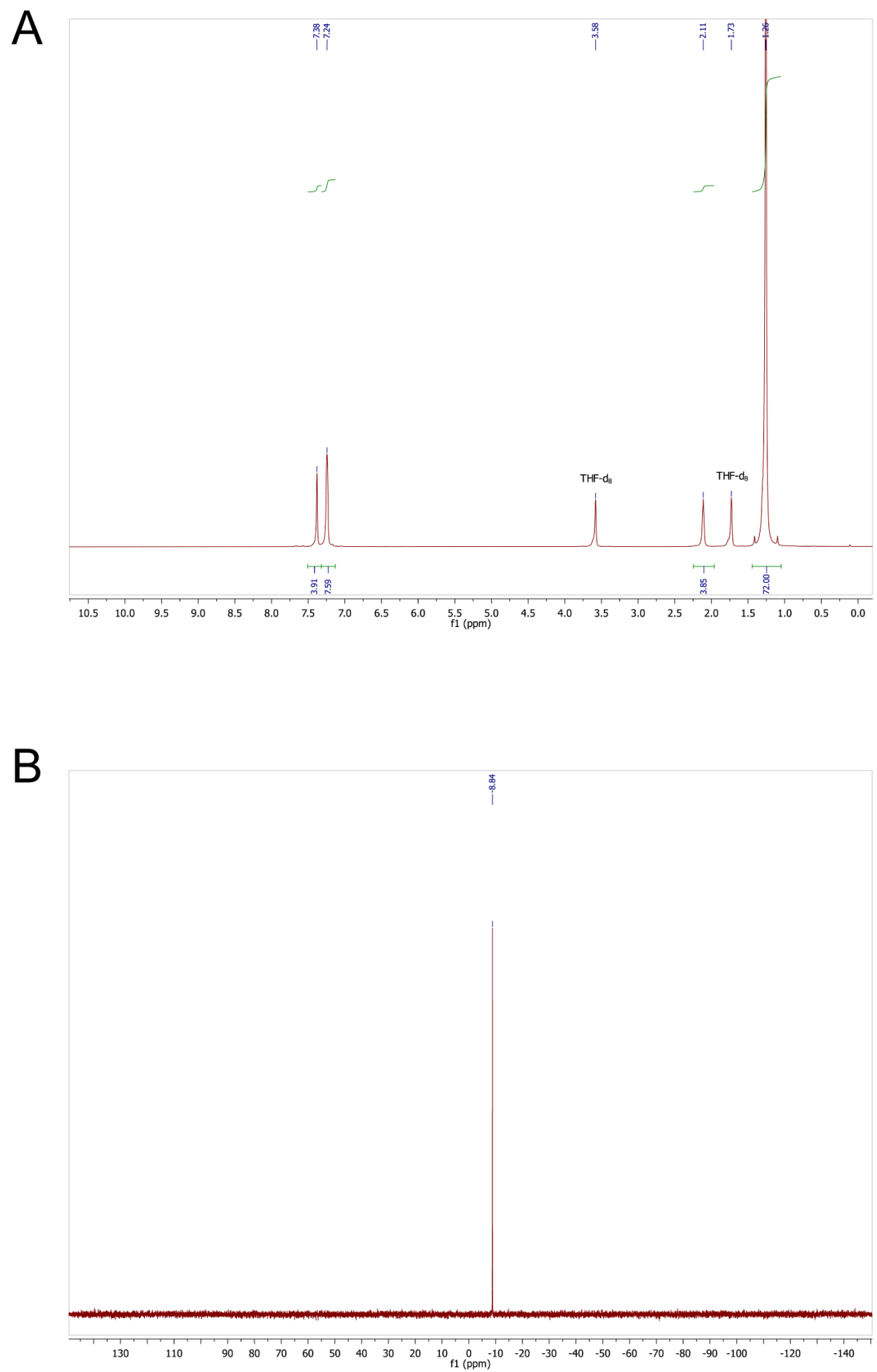

Figure S4. (A) ${ }^{1} \mathrm{H}$ NMR (400 MHz) and (B) ${ }^{31} \mathrm{P}$ NMR (400 MHz) of ${ }^{\mathrm{tBu}} \mathrm{dppe}$. 


\section{DFT Analysis}

\subsection{Calculated MO Energy Level Diagrams}
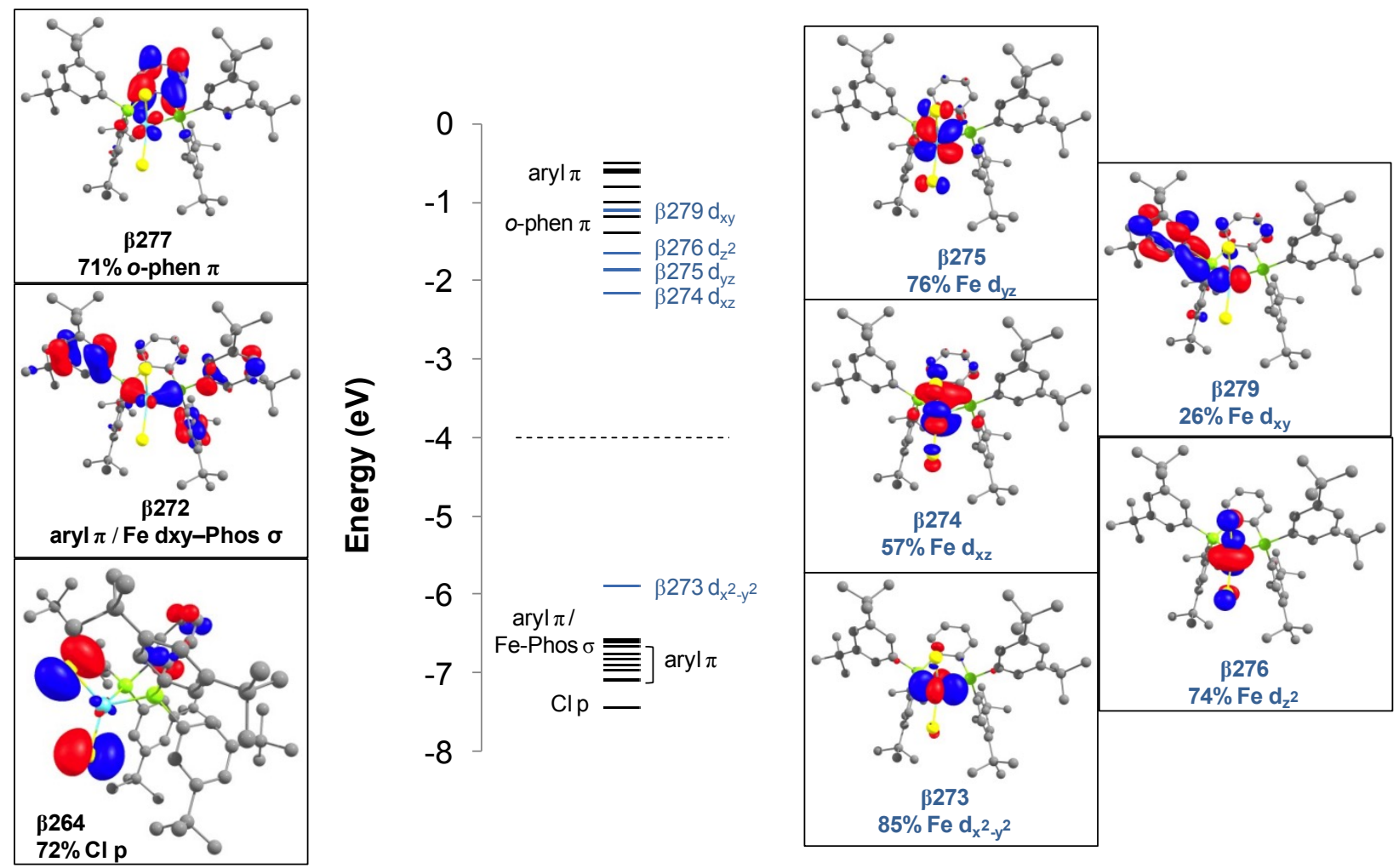

Figure S5. Calculated FMO energy level diagram for $\mathrm{FeCl}_{2}(\mathrm{SciOPP})$ and selected orbital depictions. 

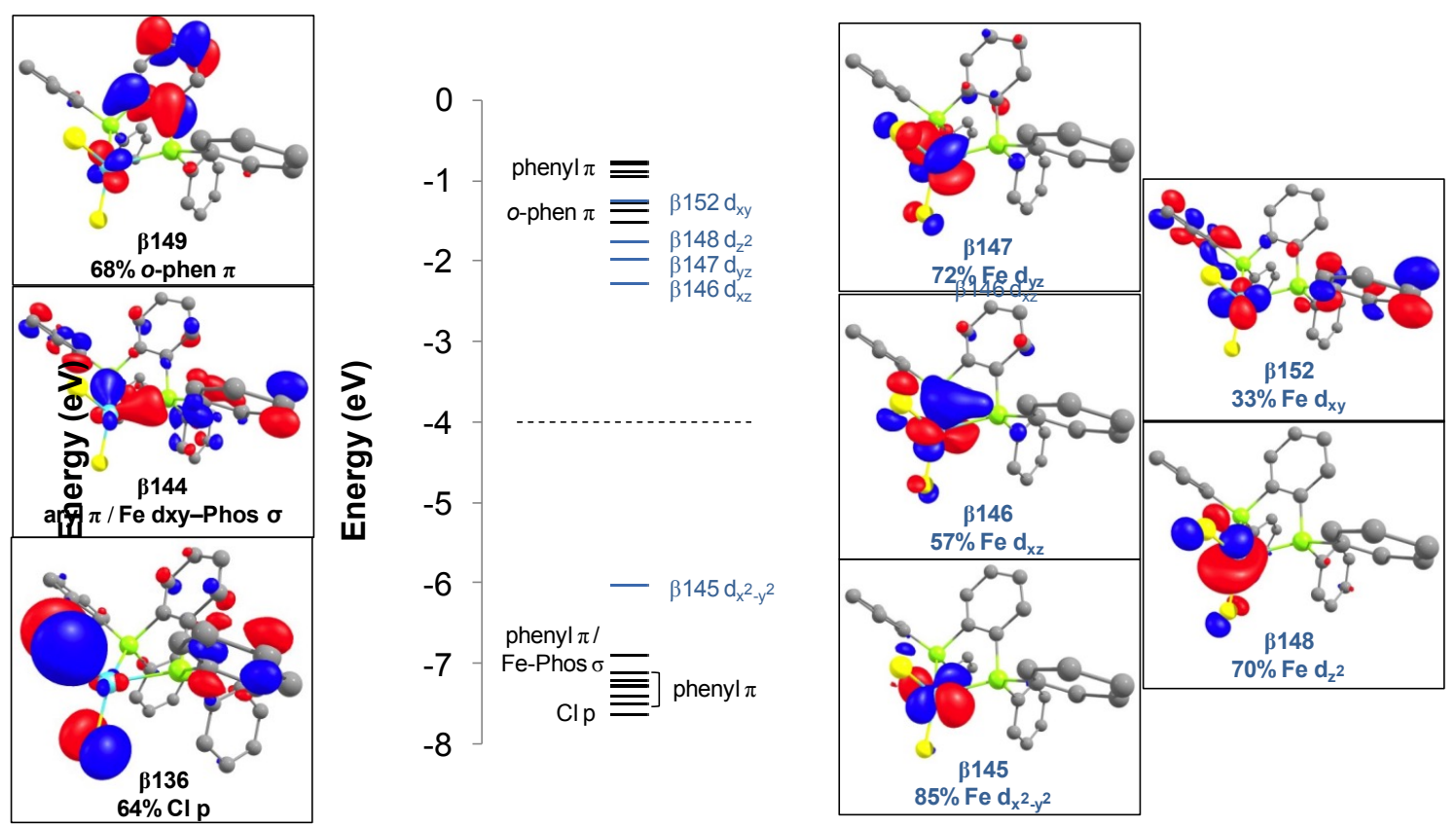

Figure S6. Calculated $\mathrm{MO}$ energy level diagram for $\mathrm{FeCl}_{2}(\mathrm{dpbz})$ and selected orbital depictions.
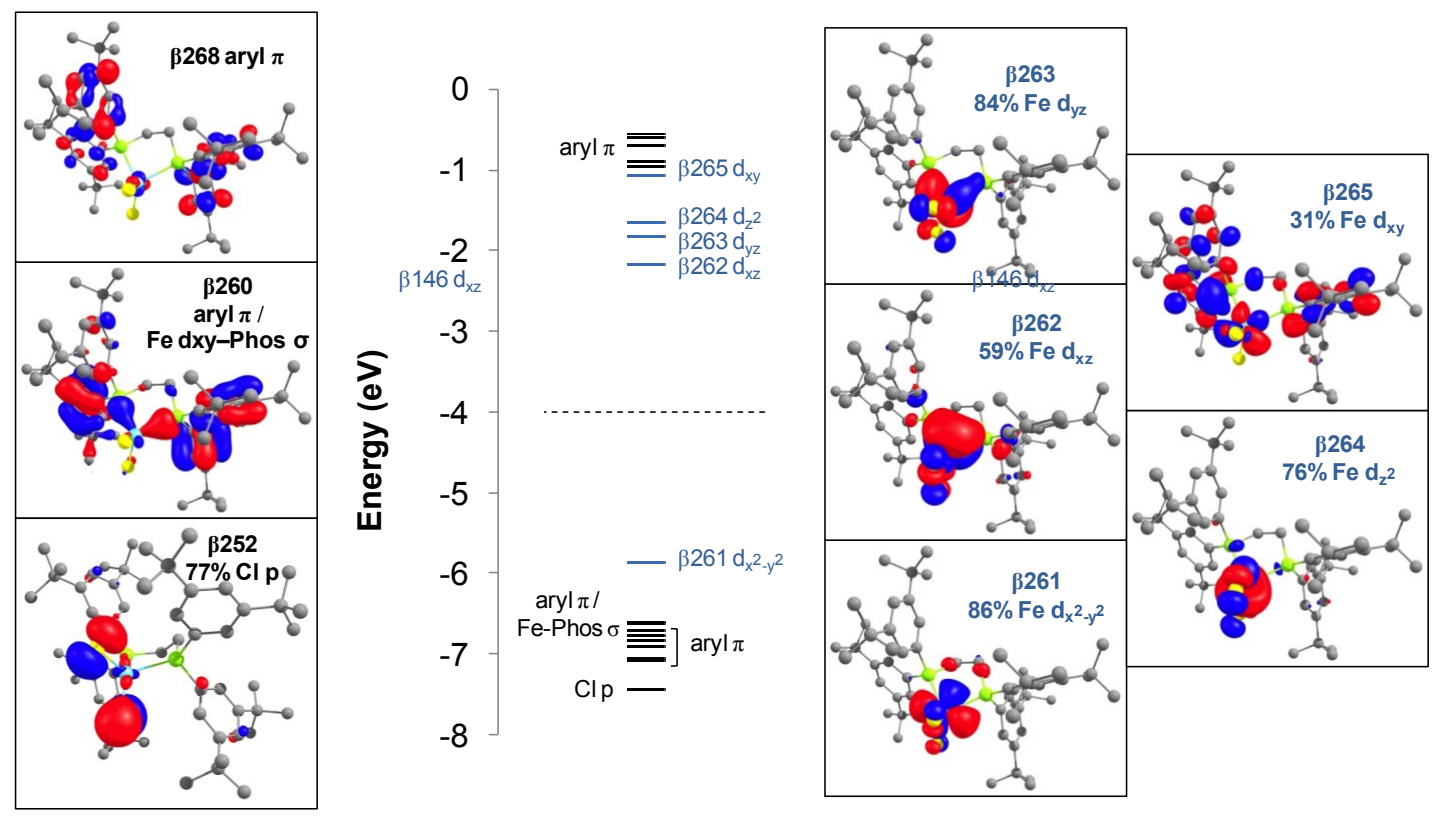

Figure S7. Calculated FMO energy level diagram for $\mathrm{FeCl}_{2}\left({ }^{\mathrm{CBu}} \mathrm{dppe}\right)$ and selected orbital depictions. 

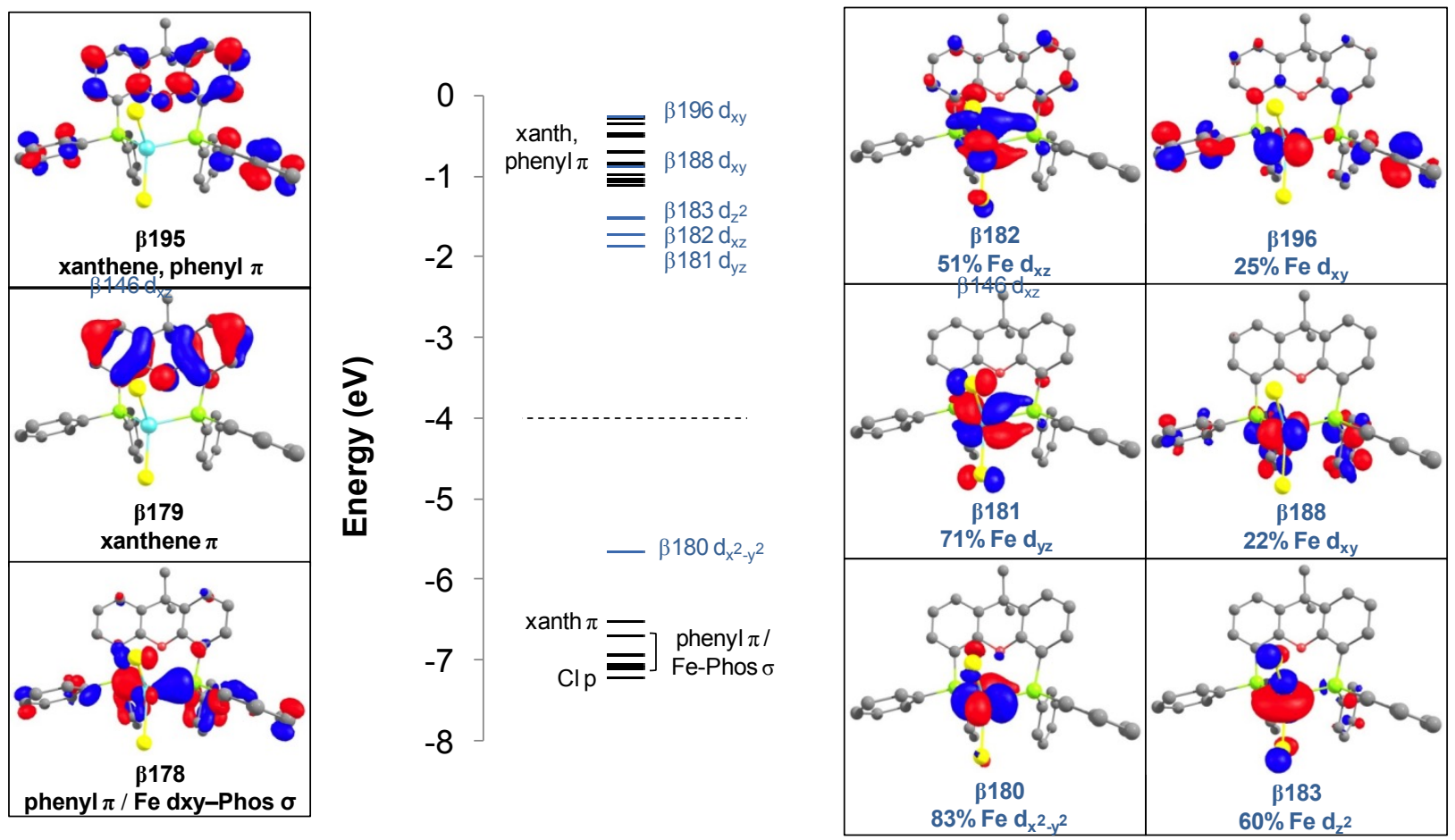

Figure S8. Calculated FMO energy level diagram for $\mathrm{FeCl}_{2}$ (Xantphos) and selected orbital depictions. 

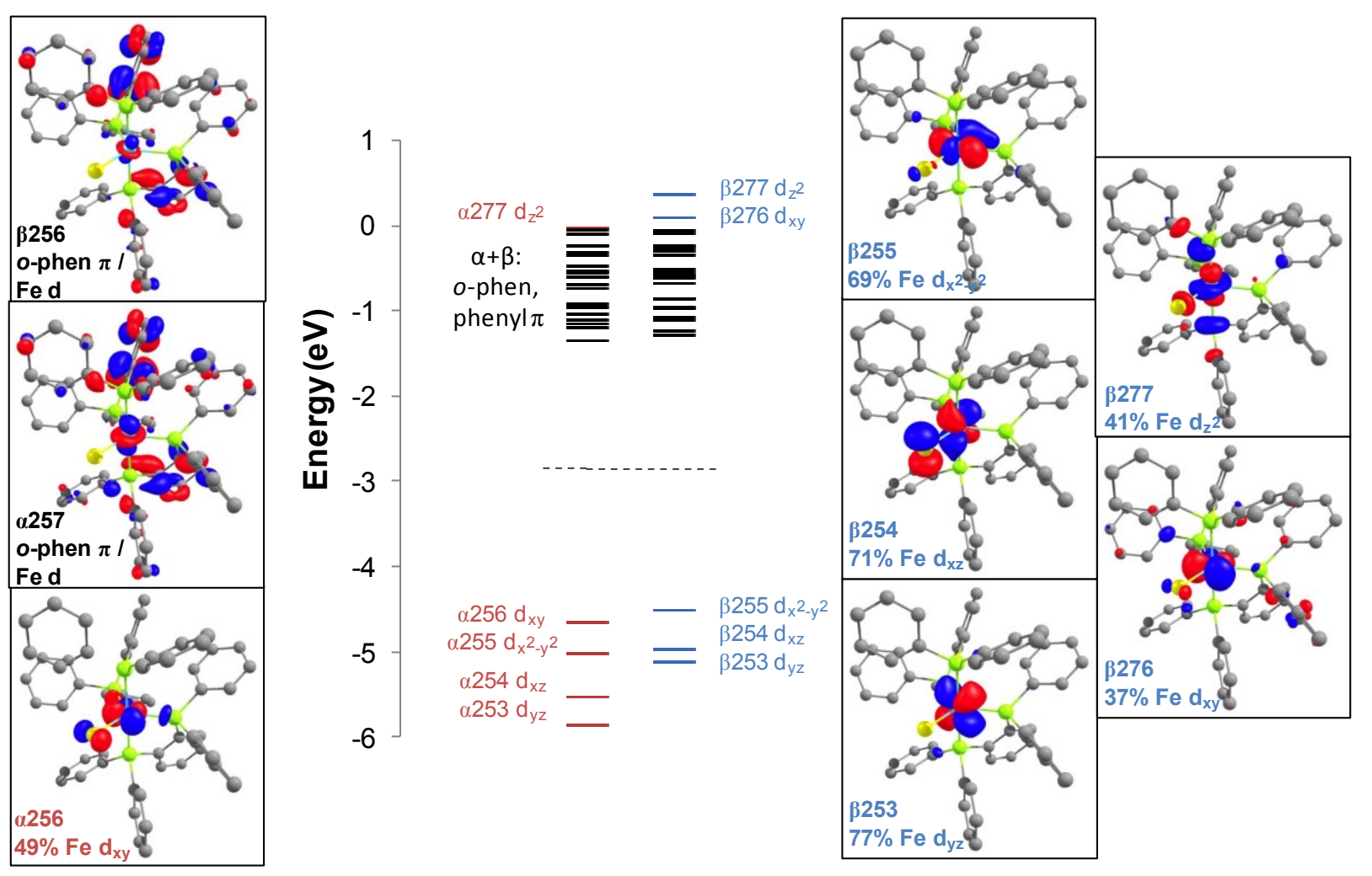

Figure S9. Calculated FMO energy level diagram for $\mathrm{FeCl}(\mathrm{dpbz})_{2}$ and selected orbital depictions. 


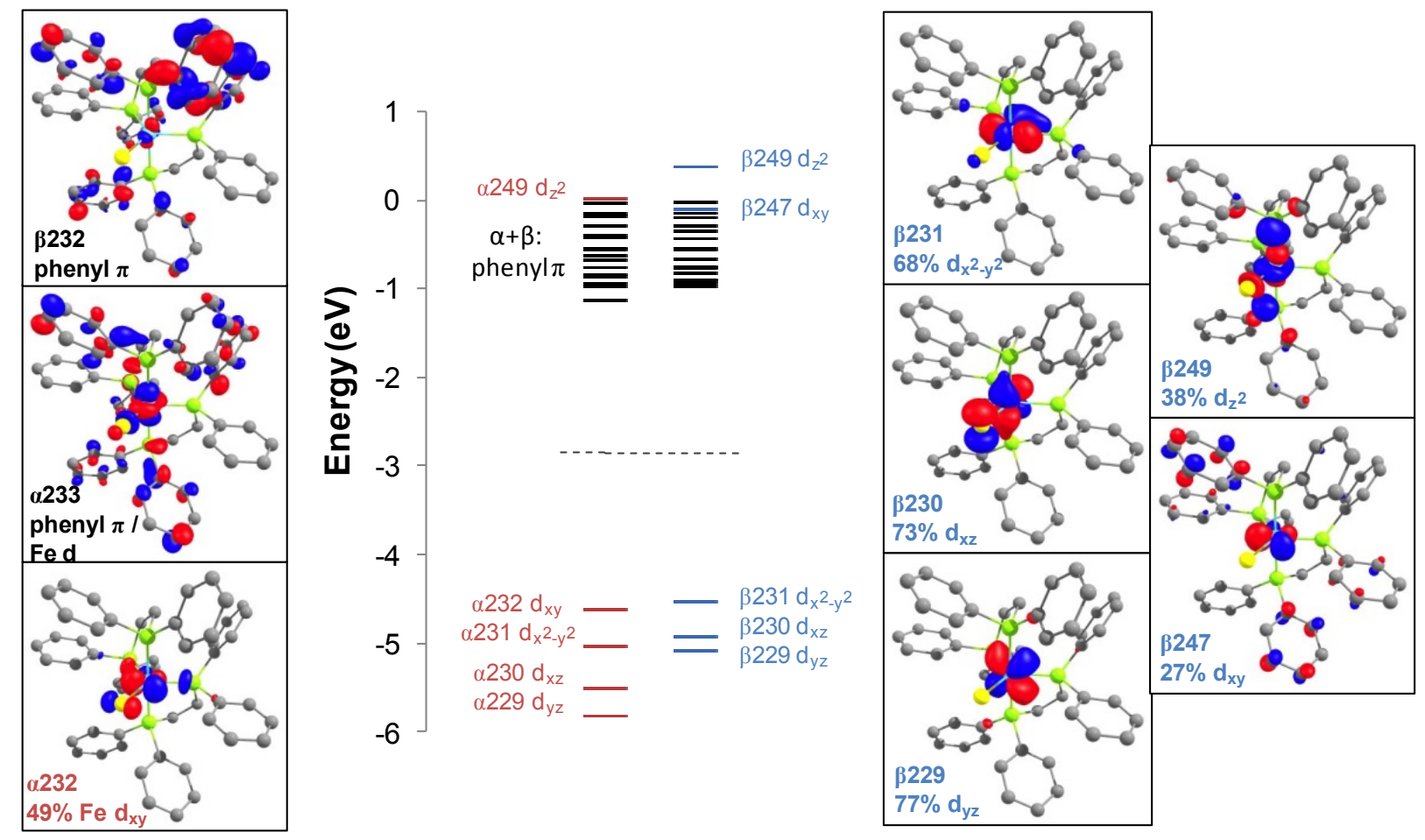

Figure S10. Calculated FMO energy level diagram for $\mathrm{FeCl}(\mathrm{dppe})_{2}$ and selected orbital depictions. 


\subsection{TD-DFT Assignments of CT Transitions for $\mathrm{FeCl}_{2}$ (bisphosphine) Complexes}

$\mathrm{FeCl}_{2}$ (SciOPP). Observed transitions in the UV-Vis MCD spectrum of $\mathrm{FeCl}_{2}(\mathrm{SciOPP})$ have been assigned using TD-DFT calculations. The high energy CT transitions observed by UV-Vis MCD are quite mixed, with dominant transition character in the bands at $27390 \mathrm{~cm}^{-1}$ and $29250 \mathrm{~cm}^{-1}$ deriving from MLCT within the $\alpha$ manifold from Fe $\mathrm{d}_{\mathrm{xy}}$ to low lying acceptor MOs of $o$-phen $\pi$ character. The band at $30590 \mathrm{~cm}^{-1}$ is composed of mixed $\alpha$ (MLCT) and $\beta$ (LMCT) character, with Fe $\mathrm{d}_{\mathrm{xy}} \rightarrow o$-phen $\pi$ character in the $\alpha$ manifold and aryl $\pi / \mathrm{Fe} \mathrm{d}_{\mathrm{xy}}$-Phos $\sigma \rightarrow \mathrm{Fe} \mathrm{d}_{\mathrm{xz}} / \mathrm{d}_{\mathrm{yz}}$ in the $\beta$ manifold. The last observed CT transition at $32685 \mathrm{~cm}^{-1}$ is again mixed but the dominant transition character is LMCT from aryl $\pi$ based MOs to $F e \mathrm{~d}_{\mathrm{xz} / \mathrm{yz}}$.

$\mathbf{F e C l}_{2}(\mathbf{d p b z})$. Observed transitions in the UV-Vis MCD spectrum of $\mathrm{FeCl}_{2}(\mathrm{dpbz})$ have been assigned using TD-DFT calculations. Assignments of the high energy CT transitions of $\mathrm{FeCl}_{2}(\mathrm{dpbz})$ are similar to those of $\mathrm{FeCl}_{2}$ (SciOPP) reflecting the consistent $o$-phen backbone structure in both complexes. The transitions observed at $27750 \mathrm{~cm}^{-1}$ and $29920 \mathrm{~cm}^{-1}$ are dominated by transitions in the $\alpha$ manifold bearing $\mathrm{Fe} \mathrm{d}_{\mathrm{xy}} \rightarrow o$-phen $\pi$ transition character. The band at $31290 \mathrm{~cm}^{-1}$ is composed of mixed $\alpha$ (MLCT) and $\beta$ (LMCT) character, with Fe $\mathrm{d}_{\mathrm{xy}} \rightarrow o$-phen $\pi$ character in the $\alpha$ manifold and phenyl $\pi / \mathrm{Fe} \mathrm{d}_{\mathrm{xy}}$-Phos $\sigma \rightarrow \mathrm{Fe}$ $\mathrm{d}_{\mathrm{xz}} / \mathrm{d}_{\mathrm{yz}}$ character in the $\beta$ manifold. The highest energy transition at $33530 \mathrm{~cm}^{-1}$ is dominantly composed of $\beta$ LMCT character in the form of phenyl $\pi / \mathrm{Fe} \mathrm{d}_{\mathrm{xy}}-\mathrm{Phos} \sigma \rightarrow \mathrm{Fe} \mathrm{d}_{\mathrm{xz}} / \mathrm{d}_{\mathrm{yz}}$.

$\mathrm{FeCl}_{2}\left({ }^{\mathrm{tBu}} \mathrm{dppe}\right)$. Observed transitions in the UV-Vis MCD spectrum of $\mathrm{FeCl}_{2}\left({ }^{\mathrm{tBu}} \mathrm{dppe}\right)$ have been assigned using TD-DFT calculations. Unlike both $\mathrm{FeCl}_{2}(\mathrm{SciOPP})$ and $\mathrm{FeCl}_{2}(\mathrm{dpbz})$, the $\mathrm{CT}$ region for $\left.\mathrm{FeCl}_{2}{ }^{\mathrm{t}}{ }^{\mathrm{Bu}} \mathrm{dppe}\right)$ is void of transitions below $30000 \mathrm{~cm}^{-1}$, reflecting the lack of backbone $\pi$-based MOs in the case of $\mathrm{FeCl}_{2}\left({ }^{\mathrm{tBu}} \mathrm{dppe}\right)$ and the fact that LLCT transitions bearing minimal Fe d character are predicted by TD-DFT to be dominant in this region. The two experimental CT transitions at $31150 \mathrm{~cm}^{-1}$ and 32670 $\mathrm{cm}^{-1}$ are both dominated by $\beta$ LMCT (aryl $\pi / \mathrm{Fe} \mathrm{d}_{\mathrm{xy}}-\mathrm{Phos} \sigma \rightarrow \mathrm{Fe} \mathrm{d}_{\mathrm{xz}} / \mathrm{d}_{\mathrm{yz}}$ ) transition character.

$\mathrm{FeCl}_{2}$ (Xantphos). Observed transitions in the UV-Vis MCD spectrum of $\mathrm{FeCl}_{2}$ (Xantphos) have been assigned using TD-DFT calculations. The high energy CT transitions observed by UV-Vis MCD are quite mixed, with dominant transition character in the band at $29330 \mathrm{~cm}^{-1}$ deriving from LMCT in the beta manifold with phenyl $\pi / \mathrm{Fe} \mathrm{d}_{\mathrm{xy}}$-Phos $\sigma \rightarrow \mathrm{Fe} \mathrm{d}_{\mathrm{xz}}$ transition character. The two higher energy transitions at $31460 \mathrm{~cm}^{-1}$ and $33280 \mathrm{~cm}^{-1}$ derive intensity from $\alpha$ MLCT to low lying ligand-based acceptor orbitals ( $\mathrm{Fe} \mathrm{d}_{\mathrm{x} 2-\mathrm{y} 2} \rightarrow$ xanth, phenyl $\pi$ ). 


\subsection{Optimized Geometry Coordinates}

\subsection{1 $\mathrm{FeCl}_{2}(\mathrm{SciOPP})$ optimized with uPBEPBE/TZVP (toluene PCM)}

$\begin{array}{llll}26 & 4.154518000 & 18.770086000 & 5.727238000 \\ 17 & 2.057797000 & 18.878341000 & 4.924956000 \\ 17 & 5.622381000 & 20.412095000 & 5.391102000 \\ 15 & 3.986997000 & 17.906150000 & 7.978061000 \\ 15 & 4.966765000 & 16.552091000 & 5.251707000 \\ 6 & 3.689280000 & 16.086356000 & 7.728498000 \\ 6 & 3.001567000 & 15.308363000 & 8.672655000 \\ 6 & 2.795523000 & 13.944309000 & 8.456212000 \\ 6 & 3.265745000 & 13.344607000 & 7.284009000 \\ 6 & 3.933564000 & 14.112248000 & 6.327703000 \\ 6 & 4.156914000 & 15.481746000 & 6.538877000 \\ 6 & 2.599075000 & 18.424342000 & 9.090831000 \\ 6 & 2.811733000 & 18.847189000 & 10.412751000 \\ 6 & 1.737011000 & 19.264249000 & 11.207662000 \\ 6 & 0.447349000 & 19.238557000 & 10.643639000 \\ 6 & 0.205748000 & 18.832115000 & 9.325095000 \\ 6 & 1.308699000 & 18.434419000 & 8.550713000 \\ 6 & 5.492435000 & 17.990361000 & 9.051227000 \\ 6 & 6.335197000 & 19.102163000 & 8.919866000 \\ 6 & 7.441241000 & 19.260789000 & 9.769896000 \\ 6 & 7.666678000 & 18.272804000 & 10.740621000 \\ 6 & 6.843296000 & 17.145071000 & 10.893457000 \\ 6 & 5.751604000 & 17.014609000 & 10.024357000 \\ 6 & 4.586666000 & 15.673345000 & 3.664898000 \\ 6 & 3.316076000 & 15.865359000 & 3.105831000 \\ 6 & 2.954369000 & 15.224208000 & 1.912298000 \\ 6 & 3.909163000 & 14.400538000 & 1.291655000 \\ 6 & 5.194789000 & 14.202015000 & 1.820295000 \\ 6 & 5.519715000 & 14.851749000 & 3.021803000 \\ 6 & 6.788079000 & 16.323172000 & 5.454259000 \\ 6 & 7.615145000 & 17.238126000 & 4.796255000 \\ 6 & 9.012183000 & 17.080223000 & 4.803574000 \\ 6 & 9.539190000 & 15.991465000 & 5.508880000 \\ 6 & 8.733514000 & 15.070940000 & 6.207216000 \\ 6 & 7.345770000 & 15.250068000 & 6.167341000 \\ 6 & 1.922191000 & 19.745820000 & 12.657597000 \\ 6 & 3.393540000 & 19.709815000 & 13.103020000 \\ 6 & 1.416636000 & 21.201279000 & 12.779793000 \\ 6 & 1.106757000 & 18.840775000 & 13.608352000 \\ 6 & -1.198371000 & 18.828167000 & 8.696684000 \\ 6 & -2.290357000 & 19.240790000 & 9.697843000 \\ 6 & -1.527149000 & 17.408801000 & 8.181721000 \\ 6 & -1.222337000 & 19.819247000 & 7.510691000 \\ 6 & 8.395515000 & 20.462924000 & 9.662307000\end{array}$




$\begin{array}{cccc}6 & 8.427704000 & 21.216796000 & 11.011030000 \\ 6 & 9.816187000 & 19.954918000 & 9.328055000 \\ 6 & 7.964255000 & 21.453207000 & 8.568008000 \\ 6 & 7.146678000 & 16.122740000 & 12.003593000 \\ 6 & 7.091849000 & 16.833676000 & 13.375031000 \\ 6 & 8.557863000 & 15.531447000 & 11.794236000 \\ 6 & 6.136601000 & 14.963214000 & 12.022363000 \\ 6 & 1.551291000 & 15.458119000 & 1.325800000 \\ 6 & 1.320216000 & 14.673142000 & 0.023595000 \\ 6 & 0.488120000 & 15.013635000 & 2.355710000 \\ 6 & 1.371137000 & 16.963820000 & 1.026302000 \\ 6 & 6.244964000 & 13.309796000 & 1.134551000 \\ 6 & 5.728578000 & 12.697671000 & -0.178276000 \\ 6 & 6.635330000 & 12.155591000 & 2.085290000 \\ 6 & 7.500580000 & 14.151979000 & 0.814168000 \\ 6 & 9.394068000 & 13.905017000 & 6.962764000 \\ 6 & 8.367794000 & 13.041478000 & 7.715533000 \\ 6 & 10.138974000 & 13.002494000 & 5.952619000 \\ 6 & 10.405650000 & 14.460493000 & 7.989817000 \\ 6 & 9.893226000 & 18.093369000 & 4.052974000 \\ 6 & 11.387511000 & 17.738726000 & 4.128210000 \\ 6 & 9.478890000 & 18.127131000 & 2.564151000 \\ 6 & 9.690630000 & 19.496192000 & 4.668365000 \\ 1 & 2.605566000 & 15.781892000 & 9.573387000 \\ 1 & 2.256516000 & 13.352302000 & 9.198588000 \\ 1 & 3.097676000 & 12.280715000 & 7.105213000 \\ 1 & 4.267134000 & 13.649851000 & 5.396315000 \\ 1 & 3.824324000 & 18.848298000 & 10.813357000 \\ 1 & -0.394728000 & 19.557438000 & 11.258385000 \\ 1 & 1.164445000 & 18.143969000 & 7.507901000 \\ 1 & 6.128117000 & 19.835263000 & 8.139043000 \\ 1 & 8.524141000 & 18.385020000 & 11.408319000 \\ 1 & 5.090278000 & 16.152755000 & 10.105116000 \\ 1 & 2.613426000 & 16.536431000 & 3.605370000 \\ 1 & 3.644228000 & 13.904379000 & 0.359820000 \\ 1 & 6.510351000 & 14.725276000 & 3.461398000 \\ 1 & 7.162917000 & 18.086684000 & 4.278390000 \\ 1 & 10.620164000 & 15.850791000 & 5.525401000 \\ 1 & 6.686127000 & 14.556138000 & 6.687296000 \\ 1 & 3.808358000 & 18.690977000 & 13.067054000 \\ 1 & 3.470942000 & 20.062834000 & 14.142171000 \\ 1 & 4.027171000 & 20.360904000 & 12.482336000 \\ 1 & 1.981378000 & 21.869956000 & 12.113260000 \\ 1 & 1.541174000 & 21.560414000 & 13.813196000 \\ 1 & 0.351675000 & 21.286984000 & 12.520221000 \\ 1 & 0.035009000 & 18.851800000 & 13.363488000 \\ 1 & 1.217831000 & 19.185123000 & 14.648367000 \\ 1 & 1.455553000 & 17.798367000 & 13.554017000\end{array}$




\begin{tabular}{lccc}
1 & -2.337121000 & 18.559129000 & 10.561082000 \\
1 & -3.270905000 & 19.213116000 & 9.199717000 \\
1 & -2.140053000 & 20.264792000 & 10.071827000 \\
1 & -0.812557000 & 17.076369000 & 7.415639000 \\
1 & -2.531795000 & 17.394579000 & 7.731353000 \\
1 & -1.510341000 & 16.677411000 & 9.004158000 \\
1 & -1.005891000 & 20.842884000 & 7.851501000 \\
1 & -2.217225000 & 19.818229000 & 7.038387000 \\
1 & -0.481295000 & 19.556298000 & 6.742257000 \\
1 & 7.427200000 & 21.590529000 & 11.276272000 \\
1 & 9.108125000 & 22.079642000 & 10.943187000 \\
1 & 8.779626000 & 20.576801000 & 11.833038000 \\
1 & 10.200488000 & 19.276182000 & 10.103325000 \\
1 & 10.511897000 & 20.804713000 & 9.248228000 \\
1 & 9.822802000 & 19.414070000 & 8.369997000 \\
1 & 7.944814000 & 20.989156000 & 7.571414000 \\
1 & 8.676370000 & 22.291296000 & 8.532658000 \\
1 & 6.966705000 & 21.873207000 & 8.765642000 \\
1 & 7.830484000 & 17.645079000 & 13.444362000 \\
1 & 7.303664000 & 16.116050000 & 14.182820000 \\
1 & 6.096489000 & 17.267793000 & 13.553786000 \\
1 & 8.622851000 & 15.001731000 & 10.832394000 \\
1 & 8.791177000 & 14.814026000 & 12.596335000 \\
1 & 9.333640000 & 16.310325000 & 11.804076000 \\
1 & 5.111324000 & 15.315509000 & 12.211605000 \\
1 & 6.400155000 & 14.261639000 & 12.827639000 \\
1 & 6.139731000 & 14.400626000 & 11.076402000 \\
1 & 2.026410000 & 14.970915000 & -0.766189000 \\
1 & 0.304603000 & 14.874802000 & -0.348060000 \\
1 & 1.408043000 & 13.586532000 & 0.176167000 \\
1 & 0.591298000 & 13.943382000 & 2.591078000 \\
1 & -0.522438000 & 15.179927000 & 1.951171000 \\
1 & 0.571071000 & 15.578880000 & 3.294656000 \\
1 & 1.483821000 & 17.577038000 & 1.931521000 \\
1 & 0.366391000 & 17.149136000 & 0.615367000 \\
1 & 2.111969000 & 17.308313000 & 0.289147000 \\
1 & 4.849089000 & 12.056583000 & -0.015187000 \\
1 & 6.516202000 & 12.073213000 & -0.625727000 \\
1 & 5.460808000 & 13.471378000 & -0.913471000 \\
1 & 7.058234000 & 12.531228000 & 3.028164000 \\
1 & 7.391550000 & 11.511547000 & 1.609942000 \\
1 & 5.760418000 & 11.533760000 & 2.328481000 \\
1 & 7.254541000 & 14.981040000 & 0.133983000 \\
1 & 8.263676000 & 13.524069000 & 0.328269000 \\
& 7.946301000 & 14.582200000 & 1.722390000 \\
\hline & 8.806188000 & 13.627856000 & 8.458661000 \\
\hline
\end{tabular}




$\begin{array}{cccc}1 & 9.442610000 & 12.583863000 & 5.210116000 \\ 1 & 10.624283000 & 12.164600000 & 6.477005000 \\ 1 & 10.918338000 & 13.556484000 & 5.409588000 \\ 1 & 11.202973000 & 15.043334000 & 7.506888000 \\ 1 & 10.881165000 & 13.632338000 & 8.537730000 \\ 1 & 9.906132000 & 15.114919000 & 8.719453000 \\ 1 & 11.757041000 & 17.732861000 & 5.164857000 \\ 1 & 11.969651000 & 18.489853000 & 3.574078000 \\ 1 & 11.599718000 & 16.756510000 & 3.678659000 \\ 1 & 9.604390000 & 17.139094000 & 2.095408000 \\ 1 & 10.104531000 & 18.848487000 & 2.016013000 \\ 1 & 8.430259000 & 18.431109000 & 2.438517000 \\ 1 & 8.640830000 & 19.818370000 & 4.620119000 \\ 1 & 10.298758000 & 20.237065000 & 4.126223000 \\ 1 & 9.998663000 & 19.508192000 & 5.724937000\end{array}$

Energy $=-5278.051650$ A.U. 


\subsection{2 $\mathrm{FeCl}_{2}(\mathrm{dpbz})$ optimized with uPBEPBE/TZVP (toluene PCM)}

$\begin{array}{llll}26 & 2.479975000 & 7.459875000 & 3.755326000 \\ 17 & 1.656390000 & 9.162622000 & 2.549976000 \\ 17 & 1.238534000 & 6.184538000 & 5.089910000 \\ 15 & 3.994621000 & 6.275499000 & 2.299654000 \\ 15 & 4.549407000 & 8.230564000 & 4.721576000 \\ 6 & 5.466720000 & 7.403814000 & 2.193664000 \\ 6 & 6.300528000 & 7.443741000 & 1.067295000 \\ 1 & 6.093814000 & 6.786394000 & 0.220400000 \\ 6 & 7.375952000 & 8.333805000 & 1.013242000 \\ 1 & 8.015028000 & 8.359223000 & 0.128494000 \\ 6 & 7.622472000 & 9.198132000 & 2.083732000 \\ 1 & 8.455474000 & 9.902461000 & 2.040245000 \\ 6 & 6.793595000 & 9.173044000 & 3.208125000 \\ 1 & 6.973636000 & 9.866162000 & 4.032388000 \\ 6 & 5.715614000 & 8.278755000 & 3.276974000 \\ 6 & 3.596833000 & 5.890935000 & 0.533359000 \\ 6 & 4.005336000 & 4.703579000 & -0.093738000 \\ 1 & 4.580981000 & 3.960312000 & 0.460743000 \\ 6 & 3.670708000 & 4.467340000 & -1.430785000 \\ 1 & 3.990117000 & 3.540243000 & -1.911221000 \\ 6 & 2.929286000 & 5.410633000 & -2.147665000 \\ 1 & 2.668046000 & 5.222870000 & -3.190991000 \\ 6 & 2.513479000 & 6.590998000 & -1.522300000 \\ 1 & 1.923010000 & 7.325424000 & -2.073596000 \\ 6 & 2.835877000 & 6.829857000 & -0.184783000 \\ 1 & 2.488943000 & 7.741613000 & 0.308037000 \\ 6 & 4.652747000 & 4.688512000 & 2.979207000 \\ 6 & 6.016354000 & 4.358077000 & 2.952986000 \\ 1 & 6.743701000 & 5.059728000 & 2.540394000 \\ 6 & 6.449205000 & 3.130217000 & 3.461398000 \\ 1 & 7.512308000 & 2.881694000 & 3.440851000 \\ 6 & 5.526822000 & 2.225511000 & 3.995003000 \\ 1 & 5.867822000 & 1.267385000 & 4.392168000 \\ 6 & 4.167959000 & 2.553987000 & 4.027681000 \\ 1 & 3.444207000 & 1.856802000 & 4.454109000 \\ 6 & 3.730084000 & 3.782758000 & 3.528851000 \\ 1 & 2.671921000 & 4.047377000 & 3.584382000 \\ 6 & 5.328904000 & 7.068593000 & 5.928630000 \\ 6 & 6.693901000 & 6.744355000 & 5.894378000 \\ 1 & 7.337800000 & 7.163561000 & 5.119038000 \\ 6 & 7.232359000 & 5.878811000 & 6.850673000 \\ 1 & 8.294786000 & 5.628996000 & 6.816215000 \\ 6 & 6.414381000 & 5.333326000 & 7.844415000 \\ 1 & 6.836911000 & 4.656059000 & 8.589305000 \\ 6 & 5.052681000 & 5.649282000 & 7.877368000 \\ 1 & 4.406756000 & 5.216403000 & 8.643603000\end{array}$




$\begin{array}{lccc}6 & 4.507192000 & 6.508456000 & 6.920833000 \\ 1 & 3.437420000 & 6.727977000 & 6.932081000 \\ 6 & 4.727968000 & 9.898281000 & 5.502904000 \\ 6 & 4.015956000 & 10.967363000 & 4.931295000 \\ 1 & 3.377619000 & 10.793772000 & 4.061300000 \\ 6 & 4.114348000 & 12.246278000 & 5.482969000 \\ 1 & 3.558712000 & 13.071396000 & 5.033069000 \\ 6 & 4.905504000 & 12.466402000 & 6.615678000 \\ 1 & 4.972914000 & 13.465695000 & 7.050260000 \\ 6 & 5.601276000 & 11.402124000 & 7.195779000 \\ 1 & 6.213799000 & 11.566384000 & 8.084698000 \\ 6 & 5.515357000 & 10.120342000 & 6.643296000 \\ 1 & 6.058954000 & 9.293659000 & 7.104123000\end{array}$

Energy $=-4022.254510$ A.U. 


\subsection{3 $\mathrm{FeCl}_{2}\left({ }^{\mathrm{tBu}} \mathrm{dppe}\right)$ optimized with uPBEPBE/TZVP (toluene PCM)}

$\begin{array}{lccc}26 & 8.613986000 & 6.905554000 & 8.682715000 \\ 17 & 10.832528000 & 7.137466000 & 8.774611000 \\ 17 & 7.642200000 & 6.513431000 & 6.702745000 \\ 15 & 7.597772000 & 8.664264000 & 10.045668000 \\ 15 & 7.824612000 & 5.405310000 & 10.434155000 \\ 6 & 6.731389000 & 6.456864000 & 11.539482000 \\ 1 & 5.760572000 & 6.510970000 & 11.022121000 \\ 1 & 6.548663000 & 5.961525000 & 12.504815000 \\ 6 & 7.326410000 & 7.855516000 & 11.720461000 \\ 1 & 8.317507000 & 7.808861000 & 12.198189000 \\ 1 & 6.690163000 & 8.485426000 & 12.359520000 \\ 6 & 8.523119000 & 10.206460000 & 10.483329000 \\ 6 & 7.925802000 & 11.471864000 & 10.466500000 \\ 1 & 6.877808000 & 11.563438000 & 10.178138000 \\ 6 & 8.660452000 & 12.617423000 & 10.811297000 \\ 6 & 10.006332000 & 12.453108000 & 11.174203000 \\ 1 & 10.587053000 & 13.332404000 & 11.445509000 \\ 6 & 10.636931000 & 11.197420000 & 11.188951000 \\ 6 & 9.875744000 & 10.076300000 & 10.829117000 \\ 1 & 10.345341000 & 9.091008000 & 10.785544000 \\ 6 & 5.913778000 & 9.232101000 & 9.533292000 \\ 6 & 5.621747000 & 9.255246000 & 8.167215000 \\ 1 & 6.366731000 & 8.882604000 & 7.461475000 \\ 6 & 4.379667000 & 9.725646000 & 7.699854000 \\ 6 & 3.447733000 & 10.154486000 & 8.650966000 \\ 1 & 2.477829000 & 10.517023000 & 8.309660000 \\ 6 & 3.704901000 & 10.134726000 & 10.036520000 \\ 6 & 4.953586000 & 9.671531000 & 10.462901000 \\ 1 & 5.188378000 & 9.660617000 & 11.526672000 \\ 6 & 9.158475000 & 4.757891000 & 11.541030000 \\ 6 & 10.269236000 & 4.181193000 & 10.917861000 \\ 1 & 10.331925000 & 4.199160000 & 9.827921000 \\ 6 & 11.304611000 & 3.602702000 & 11.672086000 \\ 6 & 11.193337000 & 3.643472000 & 13.067327000 \\ 1 & 11.988486000 & 3.205707000 & 13.671550000 \\ 6 & 10.100433000 & 4.235754000 & 13.728239000 \\ 6 & 9.082067000 & 4.789308000 & 12.943439000 \\ 1 & 8.222360000 & 5.248420000 & 13.429016000 \\ 6 & 6.755272000 & 3.934471000 & 10.090053000 \\ 6 & 6.591338000 & 2.910518000 & 11.038457000 \\ 1 & 7.145669000 & 2.961718000 & 11.975577000 \\ 6 & 5.742861000 & 1.830804000 & 10.775476000 \\ 6 & 5.065748000 & 1.808664000 & 9.538946000 \\ 1 & 4.405403000 & 0.967042000 & 9.326524000 \\ 6 & 5.213751000 & 2.810594000 & 8.573696000 \\ 6 & 6.079967000 & 3.880475000 & 8.868923000\end{array}$




\begin{tabular}{lccc}
1 & 6.247668000 & 4.672444000 & 8.134938000 \\
6 & 12.121427000 & 11.019285000 & 11.551640000 \\
6 & 12.780616000 & 12.336836000 & 11.993449000 \\
1 & 12.288927000 & 12.766573000 & 12.879750000 \\
1 & 12.771247000 & 13.091335000 & 11.192417000 \\
1 & 13.832492000 & 12.148227000 & 12.254935000 \\
6 & 12.253131000 & 10.003601000 & 12.709056000 \\
1 & 11.717645000 & 10.354385000 & 13.604491000 \\
1 & 13.313442000 & 9.870208000 & 12.973999000 \\
1 & 11.852104000 & 9.017504000 & 12.435292000 \\
6 & 12.879335000 & 10.483881000 & 10.315404000 \\
1 & 13.943737000 & 10.338398000 & 10.558421000 \\
1 & 12.813036000 & 11.194977000 & 9.478269000 \\
1 & 12.473730000 & 9.521517000 & 9.971690000 \\
6 & 7.971052000 & 13.993344000 & 10.781122000 \\
6 & 8.922662000 & 15.136415000 & 11.173359000 \\
1 & 9.777553000 & 15.213150000 & 10.484772000 \\
1 & 9.311278000 & 15.016041000 & 12.195887000 \\
1 & 8.380118000 & 16.092865000 & 11.135288000 \\
6 & 7.445208000 & 14.271135000 & 9.354911000 \\
1 & 8.270054000 & 14.277857000 & 8.626895000 \\
1 & 6.947392000 & 15.252770000 & 9.319100000 \\
1 & 6.717517000 & 13.512463000 & 9.033079000 \\
6 & 6.783749000 & 13.992047000 & 11.770546000 \\
1 & 7.128026000 & 13.797676000 & 12.797611000 \\
1 & 6.040151000 & 13.224342000 & 11.511010000 \\
1 & 6.277572000 & 14.969973000 & 11.757456000 \\
6 & 4.091330000 & 9.732982000 & 6.189663000 \\
6 & 2.722709000 & 10.350570000 & 5.858214000 \\
1 & 1.894360000 & 9.780059000 & 6.305132000 \\
1 & 2.574690000 & 10.346089000 & 4.768209000 \\
1 & 2.649145000 & 11.394922000 & 6.198577000 \\
6 & 5.181408000 & 10.551516000 & 5.461684000 \\
1 & 6.182605000 & 10.124123000 & 5.611533000 \\
1 & 5.197582000 & 11.592749000 & 5.817893000 \\
1 & 4.981938000 & 10.562398000 & 4.379049000 \\
6 & 4.111905000 & 8.278711000 & 5.666094000 \\
1 & 3.339728000 & 7.672053000 & 6.163627000 \\
1 & 5.085154000 & 7.796098000 & 5.835549000 \\
1 & 3.911480000 & 8.266309000 & 4.583333000 \\
6 & 2.622733000 & 10.620018000 & 11.016850000 \\
6 & 3.075144000 & 10.524200000 & 12.483808000 \\
1 & 3.959150000 & 11.148959000 & 12.682756000 \\
1 & 3.308134000 & 9.488534000 & 12.774478000 \\
& 2.266251000 & 10.877837000 & 13.140101000 \\
& 2.279400000 & 12.096009000 & 10.712964000 \\
\hline & 1.497953000 & 12.452556000 & 11.401858000
\end{tabular}




$\begin{array}{lccc}1 & 1.906958000 & 12.226191000 & 9.686866000 \\ 6 & 1.353450000 & 9.755419000 & 10.844680000 \\ 1 & 1.567014000 & 8.696689000 & 11.054679000 \\ 1 & 0.949456000 & 9.822029000 & 9.824435000 \\ 1 & 0.568705000 & 10.092061000 & 11.539794000 \\ 6 & 4.487293000 & 2.778340000 & 7.219038000 \\ 6 & 3.605102000 & 4.040131000 & 7.084671000 \\ 1 & 4.202724000 & 4.960926000 & 7.140890000 \\ 1 & 2.845495000 & 4.075567000 & 7.880475000 \\ 1 & 3.085510000 & 4.035835000 & 6.113811000 \\ 6 & 3.589480000 & 1.539678000 & 7.065102000 \\ 1 & 4.167470000 & 0.604436000 & 7.116860000 \\ 1 & 3.093748000 & 1.567958000 & 6.083436000 \\ 1 & 2.802374000 & 1.504245000 & 7.833807000 \\ 6 & 5.534012000 & 2.767439000 & 6.081455000 \\ 1 & 6.182433000 & 1.881163000 & 6.152902000 \\ 1 & 6.174073000 & 3.660743000 & 6.106304000 \\ 1 & 5.027320000 & 2.743202000 & 5.103941000 \\ 6 & 5.533355000 & 0.679685000 & 11.774058000 \\ 6 & 5.974360000 & -0.649635000 & 11.120783000 \\ 1 & 5.397197000 & -0.869150000 & 10.211077000 \\ 1 & 5.826577000 & -1.485147000 & 11.822725000 \\ 1 & 7.039098000 & -0.616873000 & 10.845411000 \\ 6 & 4.037170000 & 0.592751000 & 12.150645000 \\ 1 & 3.403042000 & 0.417220000 & 11.269831000 \\ 1 & 3.698555000 & 1.523324000 & 12.630638000 \\ 1 & 3.870821000 & -0.236954000 & 12.855280000 \\ 6 & 6.344510000 & 0.871330000 & 13.066626000 \\ 1 & 7.426588000 & 0.909364000 & 12.870381000 \\ 1 & 6.158779000 & 0.024855000 & 13.744408000 \\ 1 & 6.056637000 & 1.791168000 & 13.598199000 \\ 6 & 10.061407000 & 4.252942000 & 15.266339000 \\ 6 & 11.289669000 & 5.026059000 & 15.797554000 \\ 1 & 11.278408000 & 5.048483000 & 16.898404000 \\ 1 & 11.286591000 & 6.064340000 & 15.432916000 \\ 1 & 12.233260000 & 4.560202000 & 15.479913000 \\ 6 & 8.794225000 & 4.931438000 & 15.814046000 \\ 1 & 7.878770000 & 4.407332000 & 15.499561000 \\ 1 & 8.721093000 & 5.982242000 & 15.495243000 \\ 1 & 8.819462000 & 4.920495000 & 16.913778000 \\ 6 & 10.098740000 & 2.801820000 & 15.796741000 \\ 1 & 9.230960000 & 2.229246000 & 15.435315000 \\ 1 & 10.077325000 & 2.799494000 & 16.897615000 \\ 1 & 11.008147000 & 2.273132000 & 15.477238000 \\ 6 & 12.515802000 & 2.987524000 & 10.951022000 \\ 6 & 13.275876000 & 4.110028000 & 10.208978000 \\ 1 & 12.631416000 & 4.632905000 & 9.488494000 \\ 1 & 14.132841000 & 3.687754000 & 9.661097000\end{array}$




$\begin{array}{llll}1 & 13.657948000 & 4.858246000 & 10.919737000 \\ 6 & 12.031155000 & 1.934655000 & 9.929479000 \\ 1 & 11.380702000 & 2.375923000 & 9.161360000 \\ 1 & 11.470325000 & 1.129460000 & 10.427888000 \\ 1 & 12.894104000 & 1.484155000 & 9.415599000 \\ 6 & 13.489453000 & 2.298580000 & 11.922248000 \\ 1 & 13.921981000 & 3.005985000 & 12.645951000 \\ 1 & 14.323522000 & 1.862889000 & 11.352507000 \\ 1 & 13.004550000 & 1.482749000 & 12.480140000\end{array}$

Energy $=-5125.787596$ A.U. 


\subsection{4 $\mathrm{FeCl}_{2}$ (Xantphos) optimized with uPBEPBE/TZVP (gas phase calculation)}

$\begin{array}{lccc}26 & 5.559365000 & 3.569867000 & 4.082921000 \\ 17 & 6.572323000 & 3.556117000 & 2.097988000 \\ 17 & 5.535374000 & 1.976636000 & 5.644631000 \\ 15 & 6.464057000 & 5.525131000 & 5.239168000 \\ 15 & 3.199254000 & 3.965957000 & 3.592537000 \\ 8 & 3.635057000 & 5.561615000 & 6.009126000 \\ 6 & 5.797532000 & 5.808081000 & 6.945597000 \\ 6 & 6.608188000 & 5.985406000 & 8.075109000 \\ 1 & 7.691823000 & 6.028165000 & 7.958485000 \\ 6 & 6.036012000 & 6.077533000 & 9.345141000 \\ 1 & 6.677027000 & 6.205918000 & 10.219005000 \\ 6 & 4.650384000 & 5.994774000 & 9.506182000 \\ 1 & 4.226034000 & 6.062301000 & 10.508607000 \\ 6 & 0.638376000 & 4.046578000 & 7.484065000 \\ 1 & 0.064028000 & 4.037629000 & 8.411125000 \\ 6 & 0.216352000 & 3.256235000 & 6.411633000 \\ 1 & -0.678086000 & 2.638980000 & 6.511621000 \\ 6 & 0.936389000 & 3.242882000 & 5.215558000 \\ 1 & 0.613833000 & 2.608266000 & 4.389104000 \\ 6 & 2.094067000 & 4.020537000 & 5.079437000 \\ 6 & 2.274222000 & 5.810976000 & 8.469039000 \\ 6 & 1.752918000 & 7.236020000 & 8.129924000 \\ 1 & 2.095141000 & 7.562959000 & 7.138197000 \\ 1 & 2.115868000 & 7.959229000 & 8.875649000 \\ 1 & 0.652636000 & 7.247615000 & 8.135403000 \\ 6 & 1.763536000 & 5.417352000 & 9.861622000 \\ 1 & 2.106058000 & 4.414505000 & 10.153922000 \\ 1 & 0.665347000 & 5.433444000 & 9.890430000 \\ 1 & 2.105184000 & 6.137731000 & 10.617675000 \\ 6 & 4.409686000 & 5.738885000 & 7.141847000 \\ 6 & 3.804647000 & 5.832349000 & 8.402273000 \\ 6 & 1.780235000 & 4.850484000 & 7.382264000 \\ 6 & 2.485319000 & 4.808886000 & 6.172442000 \\ 6 & 8.287358000 & 5.362850000 & 5.536549000 \\ 6 & 9.158291000 & 6.462912000 & 5.519675000 \\ 1 & 8.769718000 & 7.462784000 & 5.317870000 \\ 6 & 10.524343000 & 6.279452000 & 5.751388000 \\ 1 & 11.196483000 & 7.139944000 & 5.731681000 \\ 6 & 11.028733000 & 5.000087000 & 6.002025000 \\ 1 & 12.096931000 & 4.858404000 & 6.178789000 \\ 6 & 10.164259000 & 3.901462000 & 6.017655000 \\ 1 & 10.553743000 & 2.898729000 & 6.204216000 \\ 6 & 8.799364000 & 4.077397000 & 5.779068000 \\ 1 & 8.127851000 & 3.214673000 & 5.781461000 \\ 6 & 6.318552000 & 7.171552000 & 4.405795000 \\ 6 & 5.871483000 & 8.322317000 & 5.070770000\end{array}$




$\begin{array}{lccc}1 & 5.592127000 & 8.270078000 & 6.124733000 \\ 6 & 5.789741000 & 9.540439000 & 4.389324000 \\ 1 & 5.439170000 & 10.430916000 & 4.915490000 \\ 6 & 6.160568000 & 9.620006000 & 3.044781000 \\ 1 & 6.100163000 & 10.573076000 & 2.515419000 \\ 6 & 6.607223000 & 8.474036000 & 2.378945000 \\ 1 & 6.894218000 & 8.527968000 & 1.326725000 \\ 6 & 6.678436000 & 7.252033000 & 3.049531000 \\ 1 & 7.009756000 & 6.355392000 & 2.519221000 \\ 6 & 2.749839000 & 5.483834000 & 2.633948000 \\ 6 & 1.668486000 & 6.304251000 & 2.986165000 \\ 1 & 1.058722000 & 6.057547000 & 3.857198000 \\ 6 & 1.365711000 & 7.434718000 & 2.221651000 \\ 1 & 0.522977000 & 8.068909000 & 2.505102000 \\ 6 & 2.134226000 & 7.748932000 & 1.098036000 \\ 1 & 1.894898000 & 8.630637000 & 0.500040000 \\ 6 & 3.213283000 & 6.932716000 & 0.744208000 \\ 1 & 3.822018000 & 7.176588000 & -0.128885000 \\ 6 & 3.528981000 & 5.809532000 & 1.510919000 \\ 1 & 4.385275000 & 5.184997000 & 1.242727000 \\ 6 & 2.465504000 & 2.588961000 & 2.592151000 \\ 6 & 1.509807000 & 2.803497000 & 1.587559000 \\ 1 & 1.168837000 & 3.816196000 & 1.364825000 \\ 6 & 0.998444000 & 1.722197000 & 0.864056000 \\ 1 & 0.258760000 & 1.898199000 & 0.080135000 \\ 6 & 1.433850000 & 0.422997000 & 1.139460000 \\ 1 & 1.035061000 & -0.419589000 & 0.570841000 \\ 6 & 2.387308000 & 0.205703000 & 2.138913000 \\ 1 & 2.738313000 & -0.805778000 & 2.352643000 \\ 6 & 2.909189000 & 1.282616000 & 2.858500000 \\ 1 & 3.663808000 & 1.110288000 & 3.630615000\end{array}$

Energy $=-4444.728015$ A.U. 


\subsubsection{FeCl(dppe) ${ }_{2}$ optimized with uPBEPBE/TZVP (toluene PCM)}

$\begin{array}{lrrr}26 & 2.737266000 & 17.791530000 & 5.350174000 \\ 17 & 2.876994000 & 17.807051000 & 3.011086000 \\ 15 & 1.293058000 & 16.641329000 & 6.609276000 \\ 15 & 3.902314000 & 15.859706000 & 5.439797000 \\ 15 & 1.577824000 & 19.723811000 & 5.305749000 \\ 15 & 4.012482000 & 18.940111000 & 6.789346000 \\ 6 & 2.189522000 & 15.263597000 & 7.586594000 \\ 1 & 2.128480000 & 15.482502000 & 8.660097000 \\ 1 & 1.624955000 & 14.336639000 & 7.415625000 \\ 6 & 3.651270000 & 15.126291000 & 7.146102000 \\ 1 & 3.989763000 & 14.081243000 & 7.188162000 \\ 1 & 4.306481000 & 15.717128000 & 7.800019000 \\ 6 & 0.048789000 & 15.583426000 & 5.674981000 \\ 6 & 0.154646000 & 15.490560000 & 4.281871000 \\ 1 & 0.934302000 & 16.060259000 & 3.767825000 \\ 6 & -0.708761000 & 14.662118000 & 3.556412000 \\ 1 & -0.609042000 & 14.597590000 & 2.470700000 \\ 6 & -1.685170000 & 13.914848000 & 4.218908000 \\ 1 & -2.359850000 & 13.267632000 & 3.654251000 \\ 6 & -1.792776000 & 13.994226000 & 5.612673000 \\ 1 & -2.552525000 & 13.410675000 & 6.137816000 \\ 6 & -0.930870000 & 14.822076000 & 6.336024000 \\ 1 & -1.030534000 & 14.881204000 & 7.422579000 \\ 6 & 0.159771000 & 17.364999000 & 7.912420000 \\ 6 & 0.582932000 & 17.557687000 & 9.238811000 \\ 1 & 1.567634000 & 17.212421000 & 9.556927000 \\ 6 & -0.243456000 & 18.181855000 & 10.178204000 \\ 1 & 0.106338000 & 18.309323000 & 11.205148000 \\ 6 & -1.513259000 & 18.633806000 & 9.807113000 \\ 1 & -2.161163000 & 19.119460000 & 10.539389000 \\ 6 & -1.945345000 & 18.454363000 & 8.489593000 \\ 1 & -2.934352000 & 18.802614000 & 8.184186000 \\ 6 & -1.119373000 & 17.828555000 & 7.552182000 \\ 1 & -1.478324000 & 17.697359000 & 6.530286000 \\ 6 & 3.469878000 & 14.441606000 & 4.296074000 \\ 6 & 2.841143000 & 13.273503000 & 4.754299000 \\ 1 & 2.570137000 & 13.161453000 & 5.804638000 \\ 6 & 2.543392000 & 12.228429000 & 3.873877000 \\ 1 & 2.054553000 & 11.328185000 & 4.252506000 \\ 6 & 2.866867000 & 12.336767000 & 2.519705000 \\ 1 & 2.635935000 & 11.520699000 & 1.831844000 \\ 6 & 3.489792000 & 13.498434000 & 2.052133000 \\ 1 & 3.746733000 & 13.595237000 & 0.995009000 \\ 6 & 3.789244000 & 14.542016000 & 2.930298000 \\ 1 & 4.263418000 & 15.446655000 & 2.550037000 \\ 6 & 5.750374000 & 15.830569000 & 5.200219000\end{array}$




\begin{tabular}{llll}
6 & 6.581064000 & 14.934667000 & 5.892231000 \\
1 & 6.161567000 & 14.239969000 & 6.621611000 \\
6 & 7.958519000 & 14.923280000 & 5.656501000 \\
1 & 8.595105000 & 14.226371000 & 6.206200000 \\
6 & 8.519808000 & 15.796714000 & 4.719195000 \\
1 & 9.596197000 & 15.785343000 & 4.535587000 \\
6 & 7.696012000 & 16.679875000 & 4.015367000 \\
1 & 8.125232000 & 17.362884000 & 3.279269000 \\
6 & 6.318920000 & 16.698255000 & 4.255699000 \\
1 & 5.674790000 & 17.388528000 & 3.708234000 \\
6 & 1.602688000 & 20.461017000 & 7.027348000 \\
1 & 1.316089000 & 21.521855000 & 7.007275000 \\
1 & 0.822088000 & 19.917182000 & 7.575766000 \\
6 & 2.969494000 & 20.259624000 & 7.696373000 \\
1 & 2.836761000 & 19.945017000 & 8.738809000 \\
1 & 3.557185000 & 21.187827000 & 7.711691000 \\
6 & -0.224597000 & 19.765377000 & 4.831132000 \\
6 & -1.122201000 & 20.686933000 & 5.394275000 \\
1 & -0.785801000 & 21.397329000 & 6.151295000 \\
6 & -2.461025000 & 20.704504000 & 4.994361000 \\
1 & -3.150310000 & 21.421512000 & 5.445980000 \\
6 & -2.916596000 & 19.811359000 & 4.018797000 \\
1 & -3.963057000 & 19.826999000 & 3.707182000 \\
6 & -2.024433000 & 18.903080000 & 3.442345000 \\
1 & -2.369576000 & 18.203473000 & 2.678147000 \\
6 & -0.686194000 & 18.878854000 & 3.847056000 \\
1 & 0.008364000 & 18.166692000 & 3.398377000 \\
6 & 2.162097000 & 21.143797000 & 4.233107000 \\
6 & 2.789396000 & 22.277150000 & 4.773174000 \\
1 & 2.965158000 & 22.358960000 & 5.846468000 \\
6 & 3.212412000 & 23.323189000 & 3.947044000 \\
1 & 3.698111000 & 24.195797000 & 4.389076000 \\
6 & 3.018291000 & 23.249733000 & 2.566035000 \\
1 & 3.347177000 & 24.066446000 & 1.920032000 \\
6 & 2.398835000 & 22.122212000 & 2.017192000 \\
1 & 2.242786000 & 22.053253000 & 0.938406000 \\
6 & 1.973935000 & 21.078000000 & 2.841371000 \\
1 & 1.501681000 & 20.200857000 & 2.399211000 \\
6 & 5.330241000 & 20.041973000 & 6.022570000 \\
6 & 5.449102000 & 20.089491000 & 4.628504000 \\
1 & 4.783922000 & 19.476202000 & 4.014324000 \\
6 & 6.388632000 & 20.930989000 & 4.022868000 \\
1 & 6.464538000 & 20.959468000 & 2.933741000 \\
6 & 7.215902000 & 21.738269000 & 4.806549000 \\
6 & 7.949145000 & 22.396323000 & 4.335183000 \\
\hline & 7.749895000 & 21.704703000 & 6.201521000 \\
\hline & 6.162213000 & 20.862099000 & 6.805118000
\end{tabular}




$\begin{array}{llll}1 & 6.089702000 & 20.836048000 & 7.895313000 \\ 6 & 5.015064000 & 18.224437000 & 8.200218000 \\ 6 & 6.346969000 & 17.829135000 & 7.979819000 \\ 1 & 6.810008000 & 18.009609000 & 7.008917000 \\ 6 & 7.090124000 & 17.214913000 & 8.991143000 \\ 1 & 8.123022000 & 16.920026000 & 8.794162000 \\ 6 & 6.518860000 & 16.978663000 & 10.245289000 \\ 1 & 7.101442000 & 16.502119000 & 11.036162000 \\ 6 & 5.194472000 & 17.360934000 & 10.477318000 \\ 1 & 4.735933000 & 17.187122000 & 11.453411000 \\ 6 & 4.451205000 & 17.972655000 & 9.463256000 \\ 1 & 3.419727000 & 18.258898000 & 9.673140000\end{array}$

Energy $=-5096.171316$ A.U. 


\subsubsection{FeCl(dpbz) $)_{2}$ optimized with uPBEPBE/TZVP (toluene PCM)}

$\begin{array}{lccc}26 & 5.064192000 & 2.433568000 & 6.643351000 \\ 15 & 3.478121000 & 1.226898000 & 5.593272000 \\ 17 & 5.177854000 & 2.684415000 & 8.955273000 \\ 6 & 7.363803000 & 1.786741000 & 4.301397000 \\ 6 & 7.931249000 & 1.777911000 & 3.019813000 \\ 1 & 7.848562000 & 2.660550000 & 2.382210000 \\ 6 & 8.591224000 & 0.636639000 & 2.552675000 \\ 1 & 9.018638000 & 0.629053000 & 1.547654000 \\ 6 & 8.709416000 & -0.488285000 & 3.374167000 \\ 1 & 9.227846000 & -1.379060000 & 3.013379000 \\ 6 & 8.158172000 & -0.478959000 & 4.659337000 \\ 1 & 8.253112000 & -1.363910000 & 5.290268000 \\ 6 & 7.466220000 & 0.648063000 & 5.120100000 \\ 6 & 7.895476000 & 4.255955000 & 5.699961000 \\ 6 & 7.622631000 & 5.300764000 & 6.597630000 \\ 1 & 6.597110000 & 5.488381000 & 6.918380000 \\ 6 & 8.654603000 & 6.100625000 & 7.094703000 \\ 1 & 8.419563000 & 6.912714000 & 7.786227000 \\ 6 & 9.979221000 & 5.855786000 & 6.718378000 \\ 1 & 10.787376000 & 6.474778000 & 7.113726000 \\ 6 & 10.262259000 & 4.806695000 & 5.839488000 \\ 1 & 11.294121000 & 4.598610000 & 5.547911000 \\ 6 & 9.228357000 & 4.014406000 & 5.330492000 \\ 1 & 9.468253000 & 3.200317000 & 4.645208000 \\ 15 & 6.453436000 & 3.236343000 & 5.059664000 \\ 15 & 6.673881000 & 0.852844000 & 6.796679000 \\ 6 & 6.049440000 & 4.218673000 & 3.507096000 \\ 6 & 4.966586000 & 3.820038000 & 2.706372000 \\ 1 & 4.352648000 & 2.970625000 & 3.005361000 \\ 6 & 4.644178000 & 4.514224000 & 1.537416000 \\ 1 & 3.796917000 & 4.184707000 & 0.932262000 \\ 6 & 5.394713000 & 5.629917000 & 1.152375000 \\ 1 & 5.140066000 & 6.177377000 & 0.242500000 \\ 6 & 6.471951000 & 6.038310000 & 1.942987000 \\ 1 & 7.066424000 & 6.907815000 & 1.653410000 \\ 6 & 6.798770000 & 5.337962000 & 3.109012000 \\ 1 & 7.642134000 & 5.673396000 & 3.713064000 \\ 15 & 3.473480000 & 4.040623000 & 6.559594000 \\ 6 & 8.209155000 & 1.129496000 & 7.845033000 \\ 6 & 8.439728000 & 2.354387000 & 8.482412000 \\ 1 & 7.686840000 & 3.137864000 & 8.425487000 \\ 6 & 9.615405000 & 2.561995000 & 9.211910000 \\ 1 & 9.776173000 & 3.522298000 & 9.706401000 \\ 6 & 10.574504000 & 1.552023000 & 9.309087000 \\ 1 & 11.491141000 & 1.715994000 & 9.879817000 \\ 6 & 10.351358000 & 0.324361000 & 8.675823000\end{array}$




$\begin{array}{lccc}1 & 11.091480000 & -0.475502000 & 8.749437000 \\ 6 & 9.176492000 & 0.114680000 & 7.951133000 \\ 1 & 9.011038000 & -0.855908000 & 7.480150000 \\ 6 & 6.230243000 & -0.861607000 & 7.364431000 \\ 6 & 6.208273000 & -1.114693000 & 8.748786000 \\ 1 & 6.511532000 & -0.332093000 & 9.446881000 \\ 6 & 5.800121000 & -2.358827000 & 9.233921000 \\ 1 & 5.790685000 & -2.540492000 & 10.310716000 \\ 6 & 5.407339000 & -3.366987000 & 8.346632000 \\ 1 & 5.092115000 & -4.340884000 & 8.726627000 \\ 6 & 5.416734000 & -3.118642000 & 6.971726000 \\ 1 & 5.105982000 & -3.896212000 & 6.270684000 \\ 6 & 5.818198000 & -1.871732000 & 6.481424000 \\ 1 & 5.802043000 & -1.689447000 & 5.406711000 \\ 6 & 2.405942000 & 3.740701000 & 5.058749000 \\ 6 & 1.604588000 & 4.691007000 & 4.413274000 \\ 1 & 1.589455000 & 5.725834000 & 4.758793000 \\ 6 & 0.831687000 & 4.324578000 & 3.307109000 \\ 1 & 0.226149000 & 5.076002000 & 2.796108000 \\ 6 & 0.838948000 & 3.001803000 & 2.854847000 \\ 1 & 0.237839000 & 2.714680000 & 1.989359000 \\ 6 & 1.610465000 & 2.041023000 & 3.516271000 \\ 1 & 1.608672000 & 1.005925000 & 3.168790000 \\ 6 & 2.397469000 & 2.404974000 & 4.617657000 \\ 6 & 3.999459000 & 5.824007000 & 6.489450000 \\ 6 & 4.300713000 & 6.457703000 & 7.710535000 \\ 1 & 4.155013000 & 5.920551000 & 8.650034000 \\ 6 & 4.785499000 & 7.766485000 & 7.728786000 \\ 1 & 5.011259000 & 8.244363000 & 8.684447000 \\ 6 & 4.981027000 & 8.461971000 & 6.529983000 \\ 1 & 5.357602000 & 9.486720000 & 6.544419000 \\ 6 & 4.694823000 & 7.835473000 & 5.314666000 \\ 1 & 4.850318000 & 8.366399000 & 4.373123000 \\ 6 & 2.119941000 & 4.189558000 & 7.858259000 \\ 6 & 1.909989000 & 3.180910000 & 8.806322000 \\ 1 & 2.591881000 & 2.334227000 & 8.849115000 \\ 6 & 0.849388000 & 3.273264000 & 9.714614000 \\ 1 & 0.705427000 & 2.479937000 & 10.451105000 \\ 6 & -0.014919000 & 4.369303000 & 9.682997000 \\ 1 & -0.841368000 & 4.439965000 & 10.393527000 \\ 6 & 0.187857000 & 5.381309000 & 8.738377000 \\ 1 & -0.478235000 & 6.246420000 & 8.707162000 \\ 6 & 1.247969000 & 5.292117000 & 7.834142000 \\ 1 & 1.402951000 & 6.099731000 & 7.116720000 \\ 6 & 4.212410000 & 6.522180000 & 5.291180000 \\ 6 & 4.011085000 & 6.044660000 & 4.332092000 \\ 6 & 2.212018000 & 0.444071000 & 6.738344000 \\ & 2.684073000 & -0.230861000 & 7.875463000\end{array}$




$\begin{array}{lrrr}1 & 3.755154000 & -0.267211000 & 8.078084000 \\ 6 & 1.793179000 & -0.839846000 & 8.763067000 \\ 1 & 2.181313000 & -1.364316000 & 9.638903000 \\ 6 & 0.414873000 & -0.765770000 & 8.538420000 \\ 1 & -0.282654000 & -1.232425000 & 9.237253000 \\ 6 & -0.064786000 & -0.081836000 & 7.417801000 \\ 1 & -1.139778000 & -0.008077000 & 7.238790000 \\ 6 & 0.826722000 & 0.515722000 & 6.521153000 \\ 1 & 0.433997000 & 1.046287000 & 5.652700000 \\ 6 & 3.665268000 & -0.161887000 & 4.340178000 \\ 6 & 4.550025000 & 0.010702000 & 3.263192000 \\ 1 & 5.132490000 & 0.927671000 & 3.180744000 \\ 6 & 4.712363000 & -0.987510000 & 2.299858000 \\ 1 & 5.406643000 & -0.829207000 & 1.471861000 \\ 6 & 3.998931000 & -2.186284000 & 2.403074000 \\ 1 & 4.128833000 & -2.970460000 & 1.654333000 \\ 6 & 3.119895000 & -2.372229000 & 3.473273000 \\ 1 & 2.556718000 & -3.303735000 & 3.564902000 \\ 6 & 2.952109000 & -1.368495000 & 4.433659000 \\ 1 & 2.262734000 & -1.530670000 & 5.262916000\end{array}$

Energy $=-5400.697242$ A.U. 


\section{X-Ray Crystallographic Data}

$3.1{ }^{\text {tBu }}$ dppe

REFERENCE NUMBER: neijk01

CRYSTAL STRUCTURE REPORT

$$
\mathrm{C}_{58} \mathrm{H}_{88} \mathrm{P}_{2}
$$

Report prepared for:

J. Kneebone, Prof. M. Neidig

November 30, 2012

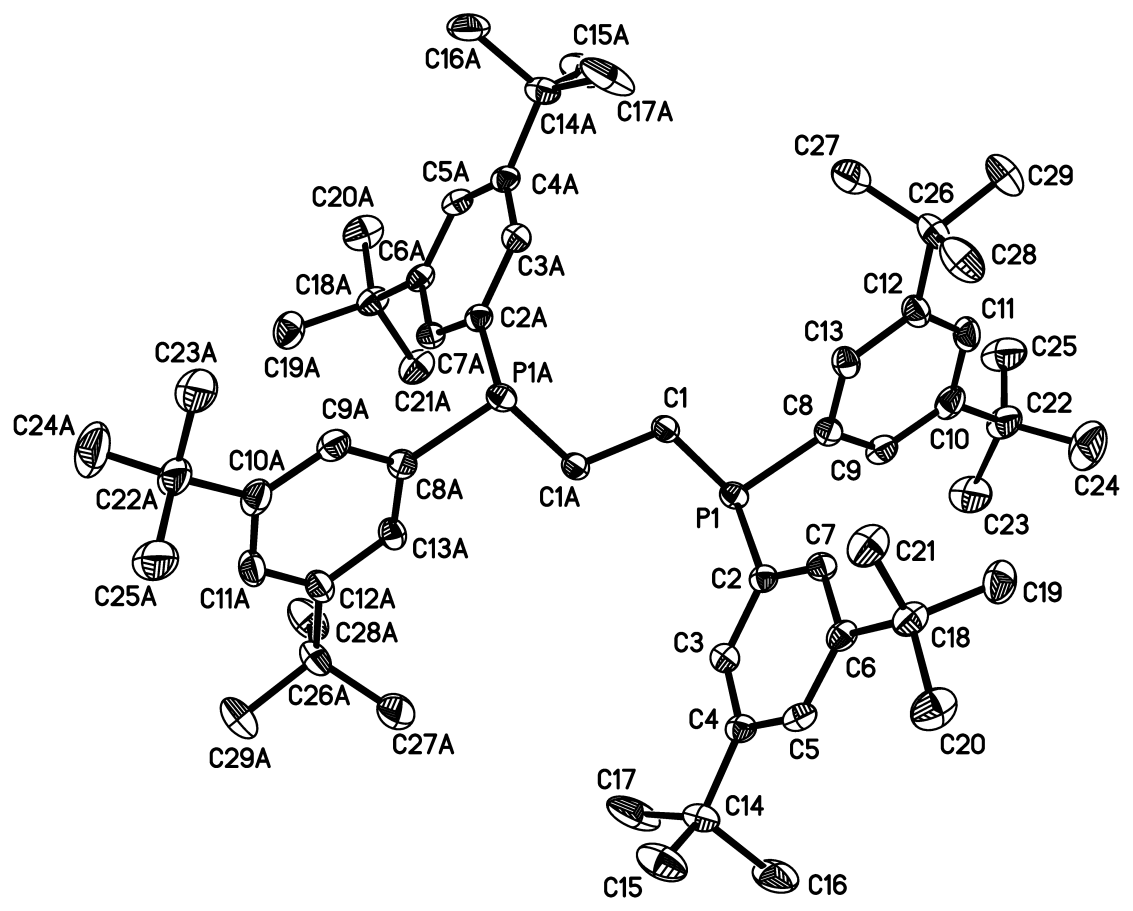

William W. Brennessel

X-ray Crystallographic Facility

Department of Chemistry, University of Rochester

120 Trustee Road

Rochester, NY 14627 


\section{Data collection}

A crystal $\left(0.38 \times 0.30 \times 0.12 \mathrm{~mm}^{3}\right)$ was placed onto the tip of a $0.1 \mathrm{~mm}$ diameter glass capillary tube or fiber and mounted on a Bruker SMART APEX II CCD Platform diffractometer for a data collection at 100.0(5) K. ${ }^{1}$ A preliminary set of cell constants and an orientation matrix were calculated from reflections harvested from three orthogonal wedges of reciprocal space. The full data collection was carried out using MoK $\alpha$ radiation (graphite monochromator) with a frame time of 45 seconds and a detector distance of $4.03 \mathrm{~cm}$. A randomly oriented region of reciprocal space was surveyed: five major sections of frames were collected with $0.50^{\circ}$ steps in $\omega$ at five different $\phi$

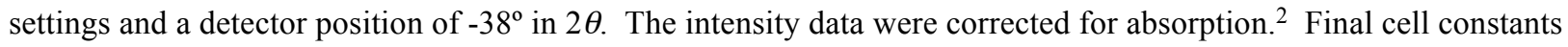
were calculated from the xyz centroids of 3989 strong reflections from the actual data collection after integration. ${ }^{3}$ See Table 1 for additional crystal and refinement information.

\section{Structure solution and refinement}

The structure was solved using SIR9 $7^{4}$ and refined using SHELXL-2012. ${ }^{5}$ The space group $P 2{ }_{1} / c$ was determined based on systematic absences. A direct-methods solution was calculated which provided most non-hydrogen atoms from the E-map. Full-matrix least squares / difference Fourier cycles were performed which located the remaining non-hydrogen atoms. All non-hydrogen atoms were refined with anisotropic displacement parameters. All hydrogen atoms were placed in ideal positions and refined as riding atoms with relative isotropic displacement parameters. The final full matrix least squares refinement converged to $R 1=0.0512\left(F^{2}, I>2 \sigma(I)\right)$ and $w R 2=$ $0.1270\left(F^{2}\right.$, all data).

\section{Structure description}

The structure is the one suggested. The molecule lies in a crystallographic inversion center; thus, one-half is unique. Four (two unique) tert-butyl groups are modeled as rotationally disordered over two positions each (70:30 and 59:41, for groups originating at atoms C14 and C22, respectively).

Unless noted otherwise all structural diagrams containing thermal displacement ellipsoids are drawn at the $50 \%$ probability level.

Data collection, structure solution, and structure refinement were conducted at the X-ray Crystallographic Facility, B51 Hutchison Hall, Department of Chemistry, University of Rochester. All publications arising from this report MUST either 1) include William W. Brennessel as a coauthor or 2) acknowledge William W. Brennessel and the Xray Crystallographic Facility of the Department of Chemistry at the University of Rochester. 
1 APEX2, version 2012.4-3; Bruker AXS: Madison, WI, 2012.

2 Sheldrick, G. M. SADABS, version 2008/1; University of Göttingen: Göttingen, Germany, 2008.

3 SAINT, version 7.68A; Bruker AXS: Madison, WI, 2009.

4 Altomare, A.; Burla, M. C.; Camalli, M.; Cascarano, G. L.; Giacovazzo, C.; Guagliardi, A.; Moliterni, A. G. G.;

Polidori, G.; Spagna, R. SIR97: A new program for solving and refining crystal structures; Istituto di Cristallografia, CNR: Bari, Italy, 1999.

5 Sheldrick, G. M. SHELXL-2012 University of Göttingen: Göttingen, Germany, 2012.

Some equations of interest:

$$
\begin{gathered}
R_{\mathrm{int}}=\Sigma\left|F_{\mathrm{o}}{ }^{2}-<F_{\mathrm{o}}{ }^{2}>\right| / \Sigma\left|F_{\mathrm{o}}{ }^{2}\right| \\
R 1=\Sigma|| F_{\mathrm{o}}|-| F_{\mathrm{c}} \| / \Sigma\left|F_{\mathrm{o}}\right| \\
w R 2=\left[\Sigma\left[w\left(F_{\mathrm{o}}{ }^{2}-F_{\mathrm{c}}{ }^{2}\right)^{2}\right] / \Sigma\left[w\left(F_{\mathrm{o}}{ }^{2}\right)^{2}\right]\right]^{1 / 2} \\
\text { where } w=1 /\left[\sigma^{2}\left(F_{\mathrm{o}}{ }^{2}\right)+(a P)^{2}+b P\right] \text { and } \\
P=1 / 3 \max \left(0, F_{\mathrm{o}}{ }^{2}\right)+2 / 3 F_{\mathrm{c}}{ }^{2} \\
\text { GOF }=S=\left[\Sigma\left[w\left(F_{\mathrm{o}}{ }^{2}-F_{\mathrm{c}}{ }^{2}\right)^{2}\right] /(m-n)\right]^{1 / 2}
\end{gathered}
$$

where $m=$ number of reflections and $n=$ number of parameters

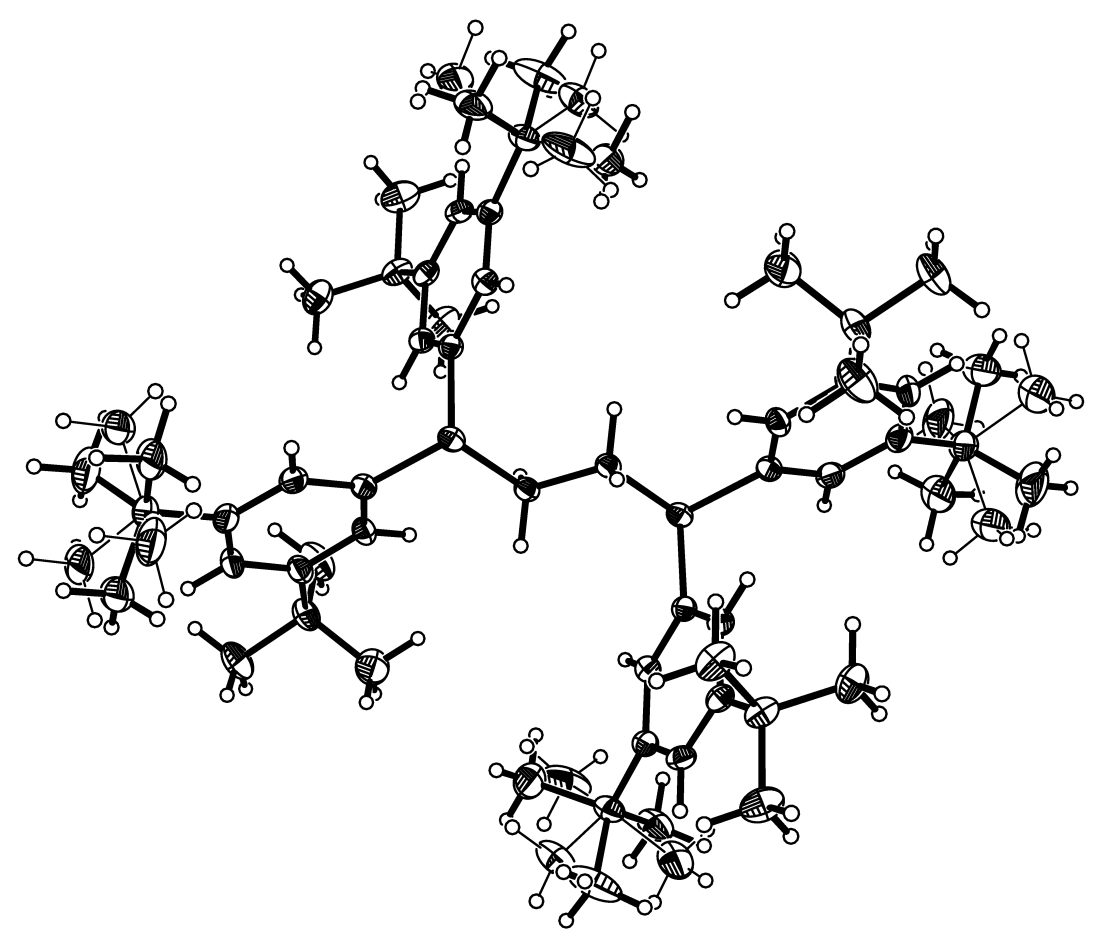




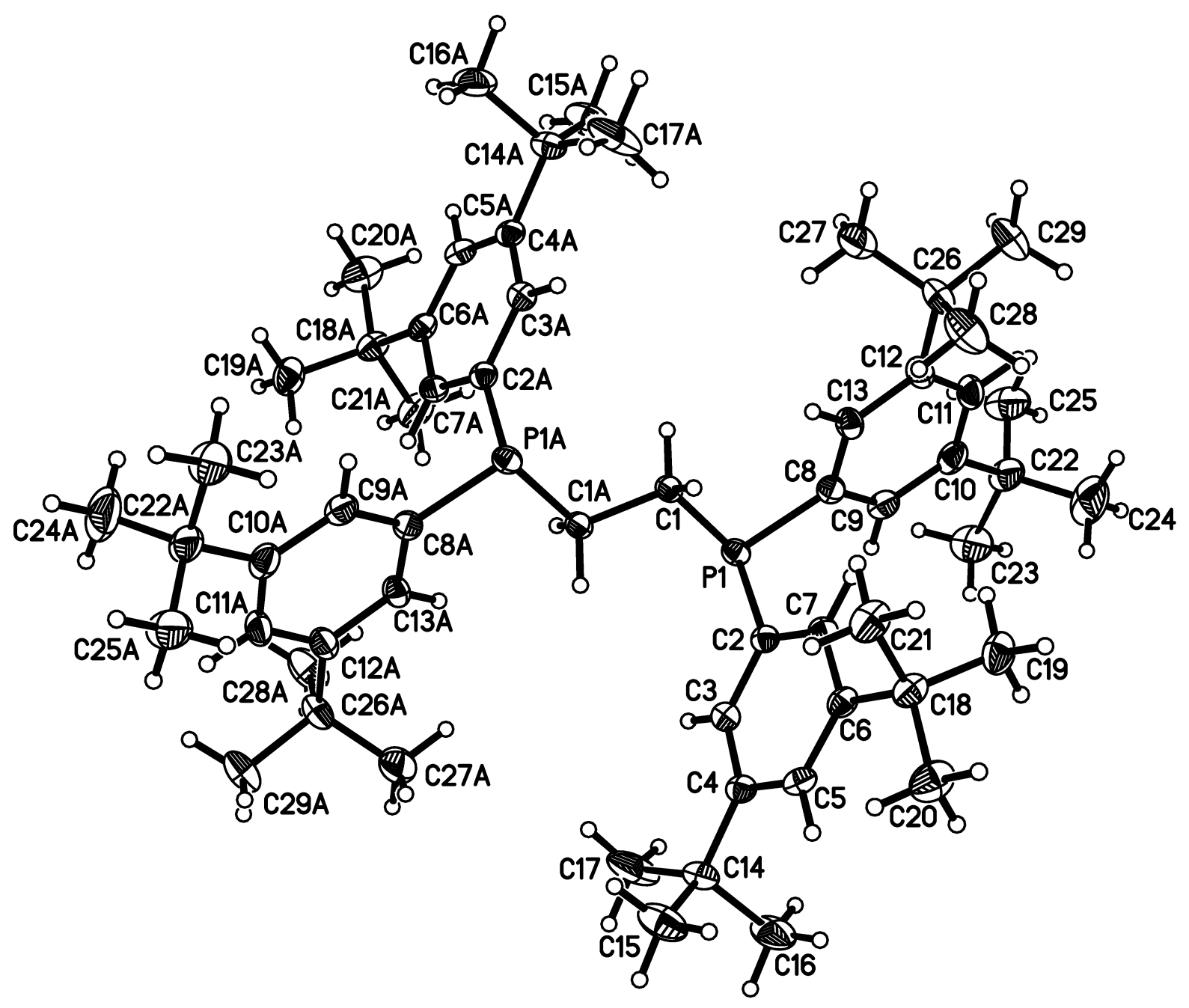


Table S1. Crystal data and structure refinement for neijk01.

\begin{tabular}{|c|c|c|}
\hline Identification code & \multicolumn{2}{|l|}{ neijk01 } \\
\hline Empirical formula & \multicolumn{2}{|l|}{ C58 H88 P2 } \\
\hline Formula weight & \multicolumn{2}{|l|}{847.22} \\
\hline Temperature & \multicolumn{2}{|l|}{$100.0(5) \mathrm{K}$} \\
\hline Wavelength & \multicolumn{2}{|l|}{$0.71073 \AA$} \\
\hline Crystal system & \multicolumn{2}{|l|}{ monoclinic } \\
\hline Space group & \multicolumn{2}{|l|}{$P 2_{1} / c$} \\
\hline \multirow[t]{3}{*}{ Unit cell dimensions } & $a=14.6204(15) \AA$ & $\alpha=90^{\circ}$ \\
\hline & $b=18.6371(19) \AA$ & $\beta=97.648(2)^{\circ}$ \\
\hline & $c=10.1420(10) \AA$ & $\gamma=90^{\circ}$ \\
\hline Volume & \multicolumn{2}{|l|}{$2738.9(5) \AA^{3}$} \\
\hline$Z$ & \multicolumn{2}{|l|}{2} \\
\hline Density (calculated) & \multicolumn{2}{|l|}{$1.027 \mathrm{Mg} / \mathrm{m}^{3}$} \\
\hline Absorption coefficient & \multicolumn{2}{|l|}{$0.113 \mathrm{~mm}^{-1}$} \\
\hline$F(000)$ & \multicolumn{2}{|l|}{932} \\
\hline Crystal color, morphology & \multicolumn{2}{|l|}{ colorless, plate } \\
\hline Crystal size & \multicolumn{2}{|c|}{$0.38 \times 0.30 \times 0.12 \mathrm{~mm}^{3}$} \\
\hline Theta range for data collection & \multicolumn{2}{|l|}{1.780 to $30.508^{\circ}$} \\
\hline Index ranges & \multicolumn{2}{|c|}{$-20 \leq h \leq 20,-26 \leq k \leq 26,-14 \leq l \leq 14$} \\
\hline Reflections collected & \multicolumn{2}{|l|}{50173} \\
\hline Independent reflections & \multicolumn{2}{|c|}{$8357[R($ int $)=0.0953]$} \\
\hline Observed reflections & \multicolumn{2}{|l|}{5438} \\
\hline Completeness to theta $=30.510^{\circ}$ & \multicolumn{2}{|l|}{$99.9 \%$} \\
\hline Absorption correction & \multicolumn{2}{|l|}{ Multi-scan } \\
\hline Max. and min. transmission & \multicolumn{2}{|l|}{0.7461 and 0.6420} \\
\hline Refinement method & \multicolumn{2}{|c|}{ Full-matrix least-squares on $F^{2}$} \\
\hline Data / restraints / parameters & \multicolumn{2}{|l|}{8357 / 18 / 345} \\
\hline Goodness-of-fit on $F^{2}$ & \multicolumn{2}{|l|}{1.023} \\
\hline Final $R$ indices $[I>2 \operatorname{sigma}(I)]$ & \multicolumn{2}{|c|}{$R 1=0.0512, w R 2=0.1080$} \\
\hline$R$ indices (all data) & \multicolumn{2}{|c|}{$R 1=0.0943, w R 2=0.1270$} \\
\hline Extinction coefficient & \multicolumn{2}{|l|}{$\mathrm{n} / \mathrm{a}$} \\
\hline Largest diff. peak and hole & \multicolumn{2}{|c|}{0.344 and -0.301 e. $\AA^{-3}$} \\
\hline
\end{tabular}


Table S2. Atomic coordinates $\left(\mathrm{x} 10^{4}\right)$ and equivalent isotropic displacement parameters $\left(\AA^{2} \times 10^{3}\right)$ for neijk01. $U_{e q}$ is defined as one third of the trace of the orthogonalized $U_{i j}$ tensor.

\begin{tabular}{|c|c|c|c|c|}
\hline & $\mathrm{x}$ & $\mathrm{y}$ & $\mathrm{z}$ & $\mathrm{U}_{\mathrm{eq}}$ \\
\hline $\mathrm{P} 1$ & $9308(1)$ & $289(1)$ & 2971(1) & $20(1)$ \\
\hline $\mathrm{C} 1$ & $9527(1)$ & $-115(1)$ & 4649(1) & 19(1) \\
\hline $\mathrm{C} 2$ & $8916(1)$ & $1178(1)$ & $3424(1)$ & $19(1)$ \\
\hline $\mathrm{C} 3$ & $9524(1)$ & 1751(1) & $3371(1)$ & $20(1)$ \\
\hline $\mathrm{C} 4$ & $9299(1)$ & $2438(1)$ & $3749(1)$ & $20(1)$ \\
\hline $\mathrm{C} 5$ & $8442(1)$ & $2537(1)$ & 4179(1) & $22(1)$ \\
\hline $\mathrm{C} 6$ & $7815(1)$ & 1983(1) & 4242(1) & $21(1)$ \\
\hline $\mathrm{C} 7$ & $8066(1)$ & 1301(1) & $3851(1)$ & $20(1)$ \\
\hline $\mathrm{C} 8$ & $8228(1)$ & $-155(1)$ & 2303(1) & $21(1)$ \\
\hline C9 & 7978(1) & $-141(1)$ & $924(2)$ & $25(1)$ \\
\hline $\mathrm{C} 10$ & 7201(1) & $-502(1)$ & $325(2)$ & $28(1)$ \\
\hline $\mathrm{C} 11$ & $6680(1)$ & $-889(1)$ & 1134(2) & $28(1)$ \\
\hline $\mathrm{C} 12$ & $6909(1)$ & $-918(1)$ & $2510(2)$ & $24(1)$ \\
\hline $\mathrm{C} 13$ & $7686(1)$ & $-541(1)$ & $3072(2)$ & $22(1)$ \\
\hline $\mathrm{C} 14$ & $9945(1)$ & $3075(1)$ & $3638(2)$ & $26(1)$ \\
\hline $\mathrm{C} 15$ & $10042(3)$ & $3527(2)$ & 4887(4) & $42(1)$ \\
\hline $\mathrm{C} 16$ & 9497(3) & $3547(2)$ & 2463(3) & $50(1)$ \\
\hline $\mathrm{C} 17$ & $10902(2)$ & $2860(2)$ & $3345(5)$ & $53(1)$ \\
\hline $\mathrm{C} 15^{\prime}$ & $9679(9)$ & $3745(5)$ & $4398(12)$ & $56(3)$ \\
\hline $\mathrm{C} 16^{\prime}$ & 9989(7) & $3245(4)$ & $2242(6)$ & $39(2)$ \\
\hline $\mathrm{C} 17^{\prime}$ & $10925(6)$ & $2850(4)$ & $4328(13)$ & $58(3)$ \\
\hline $\mathrm{C} 18$ & $6880(1)$ & 2084(1) & $4745(2)$ & $26(1)$ \\
\hline C19 & $6106(1)$ & $1806(1)$ & 3701(2) & $33(1)$ \\
\hline $\mathrm{C} 20$ & $6682(1)$ & $2870(1)$ & $5022(2)$ & $37(1)$ \\
\hline $\mathrm{C} 21$ & 6881(1) & $1655(1)$ & $6035(2)$ & $33(1)$ \\
\hline $\mathrm{C} 22$ & $6925(1)$ & $-488(1)$ & $-1191(2)$ & $36(1)$ \\
\hline $\mathrm{C} 23$ & $7662(3)$ & $-59(2)$ & $-1932(3)$ & $49(1)$ \\
\hline $\mathrm{C} 24$ & 6023(3) & $-111(2)$ & $-1557(4)$ & $57(1)$ \\
\hline $\mathrm{C} 25$ & 6924(3) & $-1242(2)$ & $-1735(3)$ & $51(1)$ \\
\hline C23' & $6007(4)$ & $-972(3)$ & $-1607(4)$ & $46(2)$ \\
\hline C24' & $7663(4)$ & $-779(4)$ & $-1861(4)$ & $54(2)$ \\
\hline
\end{tabular}




\begin{tabular}{lrrrr}
$\mathrm{C} 25^{\prime}$ & $6638(4)$ & $264(3)$ & $-1551(5)$ & $44(1)$ \\
$\mathrm{C} 26$ & $6345(1)$ & $-1336(1)$ & $3421(2)$ & $30(1)$ \\
$\mathrm{C} 27$ & $6982(1)$ & $-1824(1)$ & $4348(2)$ & $44(1)$ \\
$\mathrm{C} 28$ & $5869(1)$ & $-797(1)$ & $4248(2)$ & $42(1)$ \\
$\mathrm{C} 29$ & $5607(1)$ & $-1809(1)$ & $2643(2)$ & $40(1)$ \\
\hline
\end{tabular}


Table S3. Bond lengths $[\AA]$ and angles $\left[{ }^{\circ}\right]$ for neijk01.

\begin{tabular}{|c|c|c|c|}
\hline $\mathrm{P}(1)-\mathrm{C}(8)$ & $1.8308(15)$ & $\mathrm{C}(15)-\mathrm{H}(15 \mathrm{~A})$ & 0.9800 \\
\hline$P(1)-C(2)$ & $1.8327(15)$ & $\mathrm{C}(15)-\mathrm{H}(15 \mathrm{~B})$ & 0.9800 \\
\hline $\mathrm{P}(1)-\mathrm{C}(1)$ & $1.8491(15)$ & $\mathrm{C}(15)-\mathrm{H}(15 \mathrm{C})$ & 0.9800 \\
\hline $\mathrm{C}(1)-\mathrm{C}(1) \# 1$ & $1.529(3)$ & $\mathrm{C}(16)-\mathrm{H}(16 \mathrm{~A})$ & 0.9800 \\
\hline $\mathrm{C}(1)-\mathrm{H}(1 \mathrm{~A})$ & 0.9900 & $\mathrm{C}(16)-\mathrm{H}(16 \mathrm{~B})$ & 0.9800 \\
\hline $\mathrm{C}(1)-\mathrm{H}(1 \mathrm{~B})$ & 0.9900 & $\mathrm{C}(16)-\mathrm{H}(16 \mathrm{C})$ & 0.9800 \\
\hline$C(2)-C(7)$ & $1.3878(19)$ & $\mathrm{C}(17)-\mathrm{H}(17 \mathrm{~A})$ & 0.9800 \\
\hline $\mathrm{C}(2)-\mathrm{C}(3)$ & $1.395(2)$ & $\mathrm{C}(17)-\mathrm{H}(17 \mathrm{~B})$ & 0.9800 \\
\hline$C(3)-C(4)$ & $1.390(2)$ & $\mathrm{C}(17)-\mathrm{H}(17 \mathrm{C})$ & 0.9800 \\
\hline $\mathrm{C}(3)-\mathrm{H}(3)$ & 0.9500 & $\mathrm{C}\left(15^{\prime}\right)-\mathrm{H}(15 \mathrm{D})$ & 0.9800 \\
\hline$C(4)-C(5)$ & $1.392(2)$ & $\mathrm{C}\left(15^{\prime}\right)-\mathrm{H}(15 \mathrm{E})$ & 0.9800 \\
\hline $\mathrm{C}(4)-\mathrm{C}(14)$ & $1.529(2)$ & $\mathrm{C}\left(15^{\prime}\right)-\mathrm{H}(15 \mathrm{~F})$ & 0.9800 \\
\hline$C(5)-C(6)$ & $1.389(2)$ & $C\left(16^{\prime}\right)-H(16 D)$ & 0.9800 \\
\hline $\mathrm{C}(5)-\mathrm{H}(5)$ & 0.9500 & $\mathrm{C}\left(16^{\prime}\right)-\mathrm{H}(16 \mathrm{E})$ & 0.9800 \\
\hline$C(6)-C(7)$ & $1.395(2)$ & $\mathrm{C}\left(16^{\prime}\right)-\mathrm{H}(16 \mathrm{~F})$ & 0.9800 \\
\hline$C(6)-C(18)$ & $1.533(2)$ & $\mathrm{C}\left(17^{\prime}\right)-\mathrm{H}(17 \mathrm{D})$ & 0.9800 \\
\hline $\mathrm{C}(7)-\mathrm{H}(7)$ & 0.9500 & $\mathrm{C}\left(17^{\prime}\right)-\mathrm{H}(17 \mathrm{E})$ & 0.9800 \\
\hline $\mathrm{C}(8)-\mathrm{C}(13)$ & $1.385(2)$ & $\mathrm{C}\left(17^{\prime}\right)-\mathrm{H}(17 \mathrm{~F})$ & 0.9800 \\
\hline $\mathrm{C}(8)-\mathrm{C}(9)$ & $1.398(2)$ & $\mathrm{C}(18)-\mathrm{C}(20)$ & $1.526(2)$ \\
\hline$C(9)-C(10)$ & $1.388(2)$ & $\mathrm{C}(18)-\mathrm{C}(19)$ & $1.533(2)$ \\
\hline $\mathrm{C}(9)-\mathrm{H}(9)$ & 0.9500 & $\mathrm{C}(18)-\mathrm{C}(21)$ & $1.533(2)$ \\
\hline $\mathrm{C}(10)-\mathrm{C}(11)$ & $1.393(2)$ & $\mathrm{C}(19)-\mathrm{H}(19 \mathrm{~A})$ & 0.9800 \\
\hline $\mathrm{C}(10)-\mathrm{C}(22)$ & $1.537(2)$ & $\mathrm{C}(19)-\mathrm{H}(19 \mathrm{~B})$ & 0.9800 \\
\hline $\mathrm{C}(11)-\mathrm{C}(12)$ & $1.392(2)$ & $\mathrm{C}(19)-\mathrm{H}(19 \mathrm{C})$ & 0.9800 \\
\hline $\mathrm{C}(11)-\mathrm{H}(11)$ & 0.9500 & $\mathrm{C}(20)-\mathrm{H}(20 \mathrm{~A})$ & 0.9800 \\
\hline $\mathrm{C}(12)-\mathrm{C}(13)$ & $1.392(2)$ & $\mathrm{C}(20)-\mathrm{H}(20 \mathrm{~B})$ & 0.9800 \\
\hline$C(12)-C(26)$ & $1.531(2)$ & $\mathrm{C}(20)-\mathrm{H}(20 \mathrm{C})$ & 0.9800 \\
\hline $\mathrm{C}(13)-\mathrm{H}(13)$ & 0.9500 & $\mathrm{C}(21)-\mathrm{H}(21 \mathrm{~A})$ & 0.9800 \\
\hline $\mathrm{C}(14)-\mathrm{C}\left(16^{\prime}\right)$ & $1.461(6)$ & $\mathrm{C}(21)-\mathrm{H}(21 \mathrm{~B})$ & 0.9800 \\
\hline$C(14)-C(15)$ & $1.513(4)$ & $\mathrm{C}(21)-\mathrm{H}(21 \mathrm{C})$ & 0.9800 \\
\hline$C(14)-C(17)$ & $1.522(3)$ & $\mathrm{C}(22)-\mathrm{C}\left(24^{\prime}\right)$ & $1.455(5)$ \\
\hline$C(14)-C\left(15^{\prime}\right)$ & $1.544(9)$ & $\mathrm{C}(22)-\mathrm{C}\left(25^{\prime}\right)$ & $1.494(5)$ \\
\hline$C(14)-C(16)$ & $1.554(4)$ & $C(22)-C(24)$ & $1.496(4)$ \\
\hline $\mathrm{C}(14)-\mathrm{C}\left(17^{\prime}\right)$ & $1.566(7)$ & $C(22)-C(25)$ & $1.511(4)$ \\
\hline
\end{tabular}




\begin{tabular}{|c|c|c|c|}
\hline$C(22)-C(23)$ & $1.607(4)$ & $\mathrm{C}(1) \# 1-\mathrm{C}(1)-\mathrm{H}(1 \mathrm{~A})$ & 109.6 \\
\hline $\mathrm{C}(22)-\mathrm{C}\left(23^{\prime}\right)$ & $1.625(5)$ & $\mathrm{P}(1)-\mathrm{C}(1)-\mathrm{H}(1 \mathrm{~A})$ & 109.6 \\
\hline $\mathrm{C}(23)-\mathrm{H}(23 \mathrm{~A})$ & 0.9800 & $\mathrm{C}(1) \# 1-\mathrm{C}(1)-\mathrm{H}(1 \mathrm{~B})$ & 109.6 \\
\hline $\mathrm{C}(23)-\mathrm{H}(23 \mathrm{~B})$ & 0.9800 & $\mathrm{P}(1)-\mathrm{C}(1)-\mathrm{H}(1 \mathrm{~B})$ & 109.6 \\
\hline $\mathrm{C}(23)-\mathrm{H}(23 \mathrm{C})$ & 0.9800 & $\mathrm{H}(1 \mathrm{~A})-\mathrm{C}(1)-\mathrm{H}(1 \mathrm{~B})$ & 108.1 \\
\hline $\mathrm{C}(24)-\mathrm{H}(24 \mathrm{~A})$ & 0.9800 & $C(7)-C(2)-C(3)$ & $119.25(13)$ \\
\hline $\mathrm{C}(24)-\mathrm{H}(24 \mathrm{~B})$ & 0.9800 & $\mathrm{C}(7)-\mathrm{C}(2)-\mathrm{P}(1)$ & $123.39(11)$ \\
\hline $\mathrm{C}(24)-\mathrm{H}(24 \mathrm{C})$ & 0.9800 & $\mathrm{C}(3)-\mathrm{C}(2)-\mathrm{P}(1)$ & $117.33(11)$ \\
\hline $\mathrm{C}(25)-\mathrm{H}(25 \mathrm{~A})$ & 0.9800 & $\mathrm{C}(4)-\mathrm{C}(3)-\mathrm{C}(2)$ & $121.26(13)$ \\
\hline $\mathrm{C}(25)-\mathrm{H}(25 \mathrm{~B})$ & 0.9800 & $\mathrm{C}(4)-\mathrm{C}(3)-\mathrm{H}(3)$ & 119.4 \\
\hline $\mathrm{C}(25)-\mathrm{H}(25 \mathrm{C})$ & 0.9800 & $\mathrm{C}(2)-\mathrm{C}(3)-\mathrm{H}(3)$ & 119.4 \\
\hline $\mathrm{C}\left(23^{\prime}\right)-\mathrm{H}(23 \mathrm{D})$ & 0.9800 & $\mathrm{C}(3)-\mathrm{C}(4)-\mathrm{C}(5)$ & $117.65(13)$ \\
\hline $\mathrm{C}\left(23^{\prime}\right)-\mathrm{H}(23 \mathrm{E})$ & 0.9800 & $C(3)-C(4)-C(14)$ & $121.60(13)$ \\
\hline $\mathrm{C}\left(23^{\prime}\right)-\mathrm{H}(23 \mathrm{~F})$ & 0.9800 & $C(5)-C(4)-C(14)$ & $120.70(13)$ \\
\hline $\mathrm{C}\left(24^{\prime}\right)-\mathrm{H}(24 \mathrm{D})$ & 0.9800 & $C(6)-C(5)-C(4)$ & $122.93(14)$ \\
\hline $\mathrm{C}\left(24^{\prime}\right)-\mathrm{H}(24 \mathrm{E})$ & 0.9800 & $\mathrm{C}(6)-\mathrm{C}(5)-\mathrm{H}(5)$ & 118.5 \\
\hline $\mathrm{C}\left(24^{\prime}\right)-\mathrm{H}(24 \mathrm{~F})$ & 0.9800 & $\mathrm{C}(4)-\mathrm{C}(5)-\mathrm{H}(5)$ & 118.5 \\
\hline $\mathrm{C}\left(25^{\prime}\right)-\mathrm{H}(25 \mathrm{D})$ & 0.9800 & $\mathrm{C}(5)-\mathrm{C}(6)-\mathrm{C}(7)$ & $117.67(13)$ \\
\hline $\mathrm{C}\left(25^{\prime}\right)-\mathrm{H}(25 \mathrm{E})$ & 0.9800 & $C(5)-C(6)-C(18)$ & $123.17(13)$ \\
\hline $\mathrm{C}\left(25^{\prime}\right)-\mathrm{H}(25 \mathrm{~F})$ & 0.9800 & $C(7)-C(6)-C(18)$ & $119.15(13)$ \\
\hline$C(26)-C(29)$ & $1.529(2)$ & $C(2)-C(7)-C(6)$ & $121.25(13)$ \\
\hline$C(26)-C(27)$ & $1.532(3)$ & $\mathrm{C}(2)-\mathrm{C}(7)-\mathrm{H}(7)$ & 119.4 \\
\hline$C(26)-C(28)$ & $1.534(2)$ & $\mathrm{C}(6)-\mathrm{C}(7)-\mathrm{H}(7)$ & 119.4 \\
\hline $\mathrm{C}(27)-\mathrm{H}(27 \mathrm{~A})$ & 0.9800 & $C(13)-C(8)-C(9)$ & $118.63(14)$ \\
\hline $\mathrm{C}(27)-\mathrm{H}(27 \mathrm{~B})$ & 0.9800 & $\mathrm{C}(13)-\mathrm{C}(8)-\mathrm{P}(1)$ & $123.81(11)$ \\
\hline $\mathrm{C}(27)-\mathrm{H}(27 \mathrm{C})$ & 0.9800 & $\mathrm{C}(9)-\mathrm{C}(8)-\mathrm{P}(1)$ & $117.41(12)$ \\
\hline $\mathrm{C}(28)-\mathrm{H}(28 \mathrm{~A})$ & 0.9800 & $\mathrm{C}(10)-\mathrm{C}(9)-\mathrm{C}(8)$ & $121.28(15)$ \\
\hline $\mathrm{C}(28)-\mathrm{H}(28 \mathrm{~B})$ & 0.9800 & $\mathrm{C}(10)-\mathrm{C}(9)-\mathrm{H}(9)$ & 119.4 \\
\hline $\mathrm{C}(28)-\mathrm{H}(28 \mathrm{C})$ & 0.9800 & $\mathrm{C}(8)-\mathrm{C}(9)-\mathrm{H}(9)$ & 119.4 \\
\hline $\mathrm{C}(29)-\mathrm{H}(29 \mathrm{~A})$ & 0.9800 & $C(9)-C(10)-C(11)$ & $118.22(14)$ \\
\hline $\mathrm{C}(29)-\mathrm{H}(29 \mathrm{~B})$ & 0.9800 & $\mathrm{C}(9)-\mathrm{C}(10)-\mathrm{C}(22)$ & $121.32(16)$ \\
\hline $\mathrm{C}(29)-\mathrm{H}(29 \mathrm{C})$ & 0.9800 & $C(11)-C(10)-C(22)$ & $120.46(15)$ \\
\hline $\mathrm{C}(8)-\mathrm{P}(1)-\mathrm{C}(2)$ & $102.59(6)$ & $C(12)-C(11)-C(10)$ & $122.20(14)$ \\
\hline $\mathrm{C}(8)-\mathrm{P}(1)-\mathrm{C}(1)$ & $101.06(7)$ & $\mathrm{C}(12)-\mathrm{C}(11)-\mathrm{H}(11)$ & 118.9 \\
\hline $\mathrm{C}(2)-\mathrm{P}(1)-\mathrm{C}(1)$ & $99.14(6)$ & $\mathrm{C}(10)-\mathrm{C}(11)-\mathrm{H}(11)$ & 118.9 \\
\hline $\mathrm{C}(1) \# 1-\mathrm{C}(1)-\mathrm{P}(1)$ & $110.48(13)$ & $\mathrm{C}(11)-\mathrm{C}(12)-\mathrm{C}(13)$ & $117.74(15)$ \\
\hline
\end{tabular}




\begin{tabular}{|c|c|}
\hline $\mathrm{C}(11)-\mathrm{C}(12)-\mathrm{C}(26)$ & $123.22(14)$ \\
\hline $\mathrm{C}(13)-\mathrm{C}(12)-\mathrm{C}(26)$ & $119.03(13)$ \\
\hline$C(8)-C(13)-C(12)$ & $121.93(14)$ \\
\hline $\mathrm{C}(8)-\mathrm{C}(13)-\mathrm{H}(13)$ & 119.0 \\
\hline $\mathrm{C}(12)-\mathrm{C}(13)-\mathrm{H}(13)$ & 119.0 \\
\hline $\mathrm{C}(15)-\mathrm{C}(14)-\mathrm{C}(17)$ & $108.9(2)$ \\
\hline$C\left(16^{\prime}\right)-C(14)-C(4)$ & $110.3(3)$ \\
\hline$C(15)-C(14)-C(4)$ & $111.15(19)$ \\
\hline$C(17)-C(14)-C(4)$ & $113.75(16)$ \\
\hline $\mathrm{C}\left(16^{\prime}\right)-\mathrm{C}(14)-\mathrm{C}\left(15^{\prime}\right)$ & $110.8(5)$ \\
\hline $\mathrm{C}(4)-\mathrm{C}(14)-\mathrm{C}\left(15^{\prime}\right)$ & $113.1(4)$ \\
\hline $\mathrm{C}(15)-\mathrm{C}(14)-\mathrm{C}(16)$ & $108.0(2)$ \\
\hline $\mathrm{C}(17)-\mathrm{C}(14)-\mathrm{C}(16)$ & $107.7(2)$ \\
\hline $\mathrm{C}(4)-\mathrm{C}(14)-\mathrm{C}(16)$ & $107.15(16)$ \\
\hline $\mathrm{C}\left(16^{\prime}\right)-\mathrm{C}(14)-\mathrm{C}\left(17^{\prime}\right)$ & $109.6(5)$ \\
\hline$C(4)-C(14)-C\left(17^{\prime}\right)$ & $107.2(3)$ \\
\hline $\mathrm{C}\left(15^{\prime}\right)-\mathrm{C}(14)-\mathrm{C}\left(17^{\prime}\right)$ & $105.7(6)$ \\
\hline $\mathrm{C}(14)-\mathrm{C}(15)-\mathrm{H}(15 \mathrm{~A})$ & 109.5 \\
\hline $\mathrm{C}(14)-\mathrm{C}(15)-\mathrm{H}(15 \mathrm{~B})$ & 109.5 \\
\hline $\mathrm{H}(15 \mathrm{~A})-\mathrm{C}(15)-\mathrm{H}(15 \mathrm{~B})$ & 109.5 \\
\hline $\mathrm{C}(14)-\mathrm{C}(15)-\mathrm{H}(15 \mathrm{C})$ & 109.5 \\
\hline $\mathrm{H}(15 \mathrm{~A})-\mathrm{C}(15)-\mathrm{H}(15 \mathrm{C})$ & 109.5 \\
\hline $\mathrm{H}(15 \mathrm{~B})-\mathrm{C}(15)-\mathrm{H}(15 \mathrm{C})$ & 109.5 \\
\hline $\mathrm{C}(14)-\mathrm{C}(16)-\mathrm{H}(16 \mathrm{~A})$ & 109.5 \\
\hline $\mathrm{C}(14)-\mathrm{C}(16)-\mathrm{H}(16 \mathrm{~B})$ & 109.5 \\
\hline $\mathrm{H}(16 \mathrm{~A})-\mathrm{C}(16)-\mathrm{H}(16 \mathrm{~B})$ & 109.5 \\
\hline $\mathrm{C}(14)-\mathrm{C}(16)-\mathrm{H}(16 \mathrm{C})$ & 109.5 \\
\hline$H(16 A)-C(16)-H(16 C)$ & 109.5 \\
\hline $\mathrm{H}(16 \mathrm{~B})-\mathrm{C}(16)-\mathrm{H}(16 \mathrm{C})$ & 109.5 \\
\hline $\mathrm{C}(14)-\mathrm{C}(17)-\mathrm{H}(17 \mathrm{~A})$ & 109.5 \\
\hline $\mathrm{C}(14)-\mathrm{C}(17)-\mathrm{H}(17 \mathrm{~B})$ & 109.5 \\
\hline $\mathrm{H}(17 \mathrm{~A})-\mathrm{C}(17)-\mathrm{H}(17 \mathrm{~B})$ & 109.5 \\
\hline $\mathrm{C}(14)-\mathrm{C}(17)-\mathrm{H}(17 \mathrm{C})$ & 109.5 \\
\hline $\mathrm{H}(17 \mathrm{~A})-\mathrm{C}(17)-\mathrm{H}(17 \mathrm{C})$ & 109.5 \\
\hline $\mathrm{H}(17 \mathrm{~B})-\mathrm{C}(17)-\mathrm{H}(17 \mathrm{C})$ & 109.5 \\
\hline $\mathrm{C}(14)-\mathrm{C}\left(15^{\prime}\right)-\mathrm{H}(15 \mathrm{D})$ & 109.5 \\
\hline
\end{tabular}

\begin{tabular}{|c|c|}
\hline$C(14)-C\left(15^{\prime}\right)-H(15 E)$ & 109.5 \\
\hline $\mathrm{H}(15 \mathrm{D})-\mathrm{C}\left(15^{\prime}\right)-\mathrm{H}(15 \mathrm{E})$ & 109.5 \\
\hline $\mathrm{C}(14)-\mathrm{C}\left(15^{\prime}\right)-\mathrm{H}(15 \mathrm{~F})$ & 109.5 \\
\hline $\mathrm{H}(15 \mathrm{D})-\mathrm{C}\left(15^{\prime}\right)-\mathrm{H}(15 \mathrm{~F})$ & 109.5 \\
\hline $\mathrm{H}(15 \mathrm{E})-\mathrm{C}\left(15^{\prime}\right)-\mathrm{H}(15 \mathrm{~F})$ & 109.5 \\
\hline $\mathrm{C}(14)-\mathrm{C}\left(16^{\prime}\right)-\mathrm{H}(16 \mathrm{D})$ & 109.5 \\
\hline$C(14)-C\left(16^{\prime}\right)-H(16 E)$ & 109.5 \\
\hline$H(16 D)-C\left(16^{\prime}\right)-H(16 E)$ & 109.5 \\
\hline $\mathrm{C}(14)-\mathrm{C}\left(16^{\prime}\right)-\mathrm{H}(16 \mathrm{~F})$ & 109.5 \\
\hline $\mathrm{H}(16 \mathrm{D})-\mathrm{C}\left(16^{\prime}\right)-\mathrm{H}(16 \mathrm{~F})$ & 109.5 \\
\hline $\mathrm{H}(16 \mathrm{E})-\mathrm{C}\left(16^{\prime}\right)-\mathrm{H}(16 \mathrm{~F})$ & 109.5 \\
\hline C(14)-C(17')-H(17D) & 109.5 \\
\hline$C(14)-C\left(17^{\prime}\right)-H(17 E)$ & 109.5 \\
\hline $\mathrm{H}(17 \mathrm{D})-\mathrm{C}\left(17^{\prime}\right)-\mathrm{H}(17 \mathrm{E})$ & 109.5 \\
\hline $\mathrm{C}(14)-\mathrm{C}\left(17^{\prime}\right)-\mathrm{H}(17 \mathrm{~F})$ & 109.5 \\
\hline $\mathrm{H}(17 \mathrm{D})-\mathrm{C}\left(17^{\prime}\right)-\mathrm{H}(17 \mathrm{~F})$ & 109.5 \\
\hline $\mathrm{H}(17 \mathrm{E})-\mathrm{C}\left(17^{\prime}\right)-\mathrm{H}(17 \mathrm{~F})$ & 109.5 \\
\hline $\mathrm{C}(20)-\mathrm{C}(18)-\mathrm{C}(19)$ & $108.12(14)$ \\
\hline$C(20)-C(18)-C(6)$ & $112.26(13)$ \\
\hline$C(19)-C(18)-C(6)$ & $109.71(13)$ \\
\hline $\mathrm{C}(20)-\mathrm{C}(18)-\mathrm{C}(21)$ & $108.82(14)$ \\
\hline C(19)-C(18)-C(21) & $109.26(14)$ \\
\hline$C(6)-C(18)-C(21)$ & $108.63(12)$ \\
\hline $\mathrm{C}(18)-\mathrm{C}(19)-\mathrm{H}(19 \mathrm{~A})$ & 109.5 \\
\hline C(18)-C(19)-H(19B) & 109.5 \\
\hline H(19A)-C(19)-H(19B) & 109.5 \\
\hline $\mathrm{C}(18)-\mathrm{C}(19)-\mathrm{H}(19 \mathrm{C})$ & 109.5 \\
\hline H(19A)-C(19)-H(19C) & 109.5 \\
\hline H(19B)-C(19)-H(19C) & 109.5 \\
\hline $\mathrm{C}(18)-\mathrm{C}(20)-\mathrm{H}(20 \mathrm{~A})$ & 109.5 \\
\hline $\mathrm{C}(18)-\mathrm{C}(20)-\mathrm{H}(20 \mathrm{~B})$ & 109.5 \\
\hline $\mathrm{H}(20 \mathrm{~A})-\mathrm{C}(20)-\mathrm{H}(20 \mathrm{~B})$ & 109.5 \\
\hline $\mathrm{C}(18)-\mathrm{C}(20)-\mathrm{H}(20 \mathrm{C})$ & 109.5 \\
\hline $\mathrm{H}(20 \mathrm{~A})-\mathrm{C}(20)-\mathrm{H}(20 \mathrm{C})$ & 109.5 \\
\hline $\mathrm{H}(20 \mathrm{~B})-\mathrm{C}(20)-\mathrm{H}(20 \mathrm{C})$ & 109.5 \\
\hline $\mathrm{C}(18)-\mathrm{C}(21)-\mathrm{H}(21 \mathrm{~A})$ & 109.5 \\
\hline
\end{tabular}




\begin{tabular}{|c|c|c|c|}
\hline $\mathrm{C}(18)-\mathrm{C}(21)-\mathrm{H}(21 \mathrm{~B})$ & 109.5 & $\mathrm{C}(22)-\mathrm{C}\left(23^{\prime}\right)-\mathrm{H}(23 \mathrm{E})$ & 109.5 \\
\hline $\mathrm{H}(21 \mathrm{~A})-\mathrm{C}(21)-\mathrm{H}(21 \mathrm{~B})$ & 109.5 & $\mathrm{H}(23 \mathrm{D})-\mathrm{C}\left(23^{\prime}\right)-\mathrm{H}(23 \mathrm{E})$ & 109.5 \\
\hline $\mathrm{C}(18)-\mathrm{C}(21)-\mathrm{H}(21 \mathrm{C})$ & 109.5 & $\mathrm{C}(22)-\mathrm{C}\left(23^{\prime}\right)-\mathrm{H}(23 \mathrm{~F})$ & 109.5 \\
\hline $\mathrm{H}(21 \mathrm{~A})-\mathrm{C}(21)-\mathrm{H}(21 \mathrm{C})$ & 109.5 & $\mathrm{H}(23 \mathrm{D})-\mathrm{C}\left(23^{\prime}\right)-\mathrm{H}(23 \mathrm{~F})$ & 109.5 \\
\hline $\mathrm{H}(21 \mathrm{~B})-\mathrm{C}(21)-\mathrm{H}(21 \mathrm{C})$ & 109.5 & $\mathrm{H}(23 \mathrm{E})-\mathrm{C}\left(23^{\prime}\right)-\mathrm{H}(23 \mathrm{~F})$ & 109.5 \\
\hline$C\left(24^{\prime}\right)-C(22)-C\left(25^{\prime}\right)$ & $116.0(4)$ & $\mathrm{C}(22)-\mathrm{C}\left(24^{\prime}\right)-\mathrm{H}(24 \mathrm{D})$ & 109.5 \\
\hline$C(24)-C(22)-C(25)$ & $112.8(3)$ & $\mathrm{C}(22)-\mathrm{C}\left(24^{\prime}\right)-\mathrm{H}(24 \mathrm{E})$ & 109.5 \\
\hline $\mathrm{C}\left(24^{\prime}\right)-\mathrm{C}(22)-\mathrm{C}(10)$ & $110.4(2)$ & $\mathrm{H}(24 \mathrm{D})-\mathrm{C}\left(24^{\prime}\right)-\mathrm{H}(24 \mathrm{E})$ & 109.5 \\
\hline $\mathrm{C}\left(25^{\prime}\right)-\mathrm{C}(22)-\mathrm{C}(10)$ & $106.8(2)$ & $\mathrm{C}(22)-\mathrm{C}\left(24^{\prime}\right)-\mathrm{H}(24 \mathrm{~F})$ & 109.5 \\
\hline$C(24)-C(22)-C(10)$ & $111.2(2)$ & $\mathrm{H}(24 \mathrm{D})-\mathrm{C}\left(24^{\prime}\right)-\mathrm{H}(24 \mathrm{~F})$ & 109.5 \\
\hline$C(25)-C(22)-C(10)$ & $109.62(17)$ & $\mathrm{H}(24 \mathrm{E})-\mathrm{C}\left(24^{\prime}\right)-\mathrm{H}(24 \mathrm{~F})$ & 109.5 \\
\hline$C(24)-C(22)-C(23)$ & $106.0(2)$ & $\mathrm{C}(22)-\mathrm{C}\left(25^{\prime}\right)-\mathrm{H}(25 \mathrm{D})$ & 109.5 \\
\hline$C(25)-C(22)-C(23)$ & $105.1(2)$ & $\mathrm{C}(22)-\mathrm{C}\left(25^{\prime}\right)-\mathrm{H}(25 \mathrm{E})$ & 109.5 \\
\hline$C(10)-C(22)-C(23)$ & $111.93(17)$ & $\mathrm{H}(25 \mathrm{D})-\mathrm{C}\left(25^{\prime}\right)-\mathrm{H}(25 \mathrm{E})$ & 109.5 \\
\hline $\mathrm{C}\left(24^{\prime}\right)-\mathrm{C}(22)-\mathrm{C}\left(23^{\prime}\right)$ & $108.0(3)$ & $\mathrm{C}(22)-\mathrm{C}\left(25^{\prime}\right)-\mathrm{H}(25 \mathrm{~F})$ & 109.5 \\
\hline $\mathrm{C}\left(25^{\prime}\right)-\mathrm{C}(22)-\mathrm{C}\left(23^{\prime}\right)$ & $105.2(3)$ & $\mathrm{H}(25 \mathrm{D})-\mathrm{C}\left(25^{\prime}\right)-\mathrm{H}(25 \mathrm{~F})$ & 109.5 \\
\hline $\mathrm{C}(10)-\mathrm{C}(22)-\mathrm{C}\left(23^{\prime}\right)$ & $110.3(2)$ & $\mathrm{H}(25 \mathrm{E})-\mathrm{C}\left(25^{\prime}\right)-\mathrm{H}(25 \mathrm{~F})$ & 109.5 \\
\hline $\mathrm{C}(22)-\mathrm{C}(23)-\mathrm{H}(23 \mathrm{~A})$ & 109.5 & $C(29)-C(26)-C(12)$ & $112.43(14)$ \\
\hline $\mathrm{C}(22)-\mathrm{C}(23)-\mathrm{H}(23 \mathrm{~B})$ & 109.5 & $C(29)-C(26)-C(27)$ & $107.70(15)$ \\
\hline $\mathrm{H}(23 \mathrm{~A})-\mathrm{C}(23)-\mathrm{H}(23 \mathrm{~B})$ & 109.5 & $C(12)-C(26)-C(27)$ & $109.91(13)$ \\
\hline $\mathrm{C}(22)-\mathrm{C}(23)-\mathrm{H}(23 \mathrm{C})$ & 109.5 & $C(29)-C(26)-C(28)$ & $108.60(14)$ \\
\hline $\mathrm{H}(23 \mathrm{~A})-\mathrm{C}(23)-\mathrm{H}(23 \mathrm{C})$ & 109.5 & $\mathrm{C}(12)-\mathrm{C}(26)-\mathrm{C}(28)$ & $108.49(14)$ \\
\hline $\mathrm{H}(23 \mathrm{~B})-\mathrm{C}(23)-\mathrm{H}(23 \mathrm{C})$ & 109.5 & $\mathrm{C}(27)-\mathrm{C}(26)-\mathrm{C}(28)$ & $109.69(15)$ \\
\hline $\mathrm{C}(22)-\mathrm{C}(24)-\mathrm{H}(24 \mathrm{~A})$ & 109.5 & $\mathrm{C}(26)-\mathrm{C}(27)-\mathrm{H}(27 \mathrm{~A})$ & 109.5 \\
\hline $\mathrm{C}(22)-\mathrm{C}(24)-\mathrm{H}(24 \mathrm{~B})$ & 109.5 & $\mathrm{C}(26)-\mathrm{C}(27)-\mathrm{H}(27 \mathrm{~B})$ & 109.5 \\
\hline $\mathrm{H}(24 \mathrm{~A})-\mathrm{C}(24)-\mathrm{H}(24 \mathrm{~B})$ & 109.5 & $\mathrm{H}(27 \mathrm{~A})-\mathrm{C}(27)-\mathrm{H}(27 \mathrm{~B})$ & 109.5 \\
\hline $\mathrm{C}(22)-\mathrm{C}(24)-\mathrm{H}(24 \mathrm{C})$ & 109.5 & $\mathrm{C}(26)-\mathrm{C}(27)-\mathrm{H}(27 \mathrm{C})$ & 109.5 \\
\hline $\mathrm{H}(24 \mathrm{~A})-\mathrm{C}(24)-\mathrm{H}(24 \mathrm{C})$ & 109.5 & $\mathrm{H}(27 \mathrm{~A})-\mathrm{C}(27)-\mathrm{H}(27 \mathrm{C})$ & 109.5 \\
\hline $\mathrm{H}(24 \mathrm{~B})-\mathrm{C}(24)-\mathrm{H}(24 \mathrm{C})$ & 109.5 & $\mathrm{H}(27 \mathrm{~B})-\mathrm{C}(27)-\mathrm{H}(27 \mathrm{C})$ & 109.5 \\
\hline $\mathrm{C}(22)-\mathrm{C}(25)-\mathrm{H}(25 \mathrm{~A})$ & 109.5 & $\mathrm{C}(26)-\mathrm{C}(28)-\mathrm{H}(28 \mathrm{~A})$ & 109.5 \\
\hline $\mathrm{C}(22)-\mathrm{C}(25)-\mathrm{H}(25 \mathrm{~B})$ & 109.5 & $\mathrm{C}(26)-\mathrm{C}(28)-\mathrm{H}(28 \mathrm{~B})$ & 109.5 \\
\hline $\mathrm{H}(25 \mathrm{~A})-\mathrm{C}(25)-\mathrm{H}(25 \mathrm{~B})$ & 109.5 & $\mathrm{H}(28 \mathrm{~A})-\mathrm{C}(28)-\mathrm{H}(28 \mathrm{~B})$ & 109.5 \\
\hline $\mathrm{C}(22)-\mathrm{C}(25)-\mathrm{H}(25 \mathrm{C})$ & 109.5 & $\mathrm{C}(26)-\mathrm{C}(28)-\mathrm{H}(28 \mathrm{C})$ & 109.5 \\
\hline $\mathrm{H}(25 \mathrm{~A})-\mathrm{C}(25)-\mathrm{H}(25 \mathrm{C})$ & 109.5 & $\mathrm{H}(28 \mathrm{~A})-\mathrm{C}(28)-\mathrm{H}(28 \mathrm{C})$ & 109.5 \\
\hline $\mathrm{H}(25 \mathrm{~B})-\mathrm{C}(25)-\mathrm{H}(25 \mathrm{C})$ & 109.5 & $\mathrm{H}(28 \mathrm{~B})-\mathrm{C}(28)-\mathrm{H}(28 \mathrm{C})$ & 109.5 \\
\hline $\mathrm{C}(22)-\mathrm{C}\left(23^{\prime}\right)-\mathrm{H}(23 \mathrm{D})$ & 109.5 & $\mathrm{C}(26)-\mathrm{C}(29)-\mathrm{H}(29 \mathrm{~A})$ & 109.5 \\
\hline
\end{tabular}




$\begin{array}{llll}\mathrm{C}(26)-\mathrm{C}(29)-\mathrm{H}(29 \mathrm{~B}) & 109.5 & \mathrm{H}(29 \mathrm{~A})-\mathrm{C}(29)-\mathrm{H}(29 \mathrm{C}) & 109.5 \\ \mathrm{H}(29 \mathrm{~A})-\mathrm{C}(29)-\mathrm{H}(29 \mathrm{~B}) & 109.5 & \mathrm{H}(29 \mathrm{~B})-\mathrm{C}(29)-\mathrm{H}(29 \mathrm{C}) & 109.5 \\ \mathrm{C}(26)-\mathrm{C}(29)-\mathrm{H}(29 \mathrm{C}) & 109.5 & & \end{array}$

Symmetry transformations used to generate equivalent atoms:

$\# 1-\mathrm{x}+2,-\mathrm{y},-\mathrm{z}+1$ 
Table S4. Anisotropic displacement parameters $\left(\AA^{2} \times 10^{3}\right)$ for neijk01. The anisotropic displacement factor exponent takes the form: $-2 \pi^{2}\left[h^{2} a^{* 2} U_{11}+\ldots+2 h \mathrm{k} \mathrm{a}^{*} \mathrm{~b}^{*} \mathrm{U}_{12}\right]$

\begin{tabular}{|c|c|c|c|c|c|c|}
\hline & $\mathrm{U}_{11}$ & $\mathrm{U}_{22}$ & $\mathrm{U}_{33}$ & $\mathrm{U}_{23}$ & $\mathrm{U}_{13}$ & $\mathrm{U}_{12}$ \\
\hline P1 & $20(1)$ & $19(1)$ & $21(1)$ & $-2(1)$ & $5(1)$ & $-1(1)$ \\
\hline $\mathrm{C} 1$ & $18(1)$ & $17(1)$ & $23(1)$ & $-2(1)$ & $3(1)$ & $-1(1)$ \\
\hline $\mathrm{C} 2$ & $22(1)$ & $19(1)$ & $16(1)$ & $1(1)$ & $2(1)$ & $0(1)$ \\
\hline $\mathrm{C} 3$ & $21(1)$ & $22(1)$ & $17(1)$ & $0(1)$ & $4(1)$ & $-1(1)$ \\
\hline $\mathrm{C} 4$ & $26(1)$ & $20(1)$ & $15(1)$ & $2(1)$ & $2(1)$ & $-2(1)$ \\
\hline $\mathrm{C} 5$ & $30(1)$ & $17(1)$ & $19(1)$ & $2(1)$ & $4(1)$ & $3(1)$ \\
\hline C6 & $23(1)$ & $22(1)$ & $17(1)$ & $4(1)$ & $4(1)$ & $3(1)$ \\
\hline C7 & $21(1)$ & $20(1)$ & $19(1)$ & $2(1)$ & $4(1)$ & $-1(1)$ \\
\hline $\mathrm{C} 8$ & $22(1)$ & $19(1)$ & $20(1)$ & $-3(1)$ & $2(1)$ & $1(1)$ \\
\hline C9 & $32(1)$ & $20(1)$ & $22(1)$ & $0(1)$ & $1(1)$ & $3(1)$ \\
\hline $\mathrm{C} 10$ & $35(1)$ & $25(1)$ & $22(1)$ & $-3(1)$ & $-6(1)$ & $6(1)$ \\
\hline $\mathrm{C} 11$ & $25(1)$ & $28(1)$ & $29(1)$ & $-6(1)$ & $-8(1)$ & $0(1)$ \\
\hline $\mathrm{C} 12$ & $21(1)$ & $25(1)$ & $27(1)$ & $-4(1)$ & $0(1)$ & $-2(1)$ \\
\hline $\mathrm{C} 13$ & $21(1)$ & $23(1)$ & $20(1)$ & $-5(1)$ & $0(1)$ & $-1(1)$ \\
\hline $\mathrm{C} 14$ & $33(1)$ & $21(1)$ & $24(1)$ & $-2(1)$ & $6(1)$ & $-7(1)$ \\
\hline $\mathrm{C} 15$ & $58(2)$ & $39(2)$ & $31(2)$ & $-10(1)$ & $12(2)$ & $-22(2)$ \\
\hline $\mathrm{C} 16$ & $67(2)$ & $41(2)$ & $39(2)$ & $18(2)$ & $-7(2)$ & $-28(2)$ \\
\hline $\mathrm{C} 17$ & $39(2)$ & $33(2)$ & $93(3)$ & $-17(2)$ & $30(2)$ & $-18(1)$ \\
\hline $\mathrm{C} 15^{\prime}$ & $83(8)$ & $36(5)$ & $57(6)$ & $-21(4)$ & $37(5)$ & $-28(5)$ \\
\hline C16' & $57(5)$ & $31(4)$ & $28(3)$ & $6(3)$ & $5(3)$ & $-19(4)$ \\
\hline $\mathrm{C} 17^{\prime}$ & $38(4)$ & $38(4)$ & $88(7)$ & $23(5)$ & $-24(5)$ & $-20(3)$ \\
\hline $\mathrm{C} 18$ & $26(1)$ & $26(1)$ & $27(1)$ & $6(1)$ & $9(1)$ & $5(1)$ \\
\hline C19 & $23(1)$ & $43(1)$ & $35(1)$ & $6(1)$ & $5(1)$ & $7(1)$ \\
\hline $\mathrm{C} 20$ & $38(1)$ & $34(1)$ & $43(1)$ & $3(1)$ & $18(1)$ & $12(1)$ \\
\hline $\mathrm{C} 21$ & $33(1)$ & $40(1)$ & $29(1)$ & $9(1)$ & $13(1)$ & $7(1)$ \\
\hline $\mathrm{C} 22$ & $52(1)$ & $29(1)$ & $22(1)$ & $2(1)$ & $-12(1)$ & $-1(1)$ \\
\hline $\mathrm{C} 23$ & $78(3)$ & $47(2)$ & $17(1)$ & $8(1)$ & $-7(2)$ & $-10(2)$ \\
\hline $\mathrm{C} 24$ & $62(3)$ & $60(3)$ & $40(2)$ & $7(2)$ & $-24(2)$ & $11(2)$ \\
\hline $\mathrm{C} 25$ & $94(3)$ & $35(2)$ & $22(1)$ & $-3(1)$ & $-6(2)$ & $-12(2)$ \\
\hline C23' & $61(3)$ & $49(3)$ & $23(2)$ & $6(2)$ & $-17(2)$ & $-20(3)$ \\
\hline C24' & $60(3)$ & $80(5)$ & $20(2)$ & $-9(2)$ & $-3(2)$ & $10(3)$ \\
\hline
\end{tabular}




\begin{tabular}{lllllll}
$\mathrm{C} 25^{\prime}$ & $59(3)$ & $37(3)$ & $32(2)$ & $14(2)$ & $-14(2)$ & $-9(2)$ \\
$\mathrm{C} 26$ & $23(1)$ & $34(1)$ & $32(1)$ & $-4(1)$ & $1(1)$ & $-10(1)$ \\
$\mathrm{C} 27$ & $38(1)$ & $44(1)$ & $49(1)$ & $13(1)$ & $-1(1)$ & $-15(1)$ \\
$\mathrm{C} 28$ & $36(1)$ & $51(1)$ & $40(1)$ & $-13(1)$ & $14(1)$ & $-14(1)$ \\
$\mathrm{C} 29$ & $29(1)$ & $45(1)$ & $45(1)$ & $-11(1)$ & $3(1)$ & $-15(1)$ \\
\hline
\end{tabular}


Table S5. Hydrogen coordinates $\left(\times 10^{4}\right)$ and isotropic displacement parameters $\left(\AA^{2} \times 10^{3}\right)$ for neijk01.

\begin{tabular}{|c|c|c|c|c|}
\hline & $\mathrm{x}$ & $\mathrm{y}$ & z & $\mathrm{U}(\mathrm{eq})$ \\
\hline H1A & 9041 & 39 & 5181 & 23 \\
\hline H1B & 9504 & -644 & 4574 & 23 \\
\hline H3 & 10104 & 1669 & 3071 & 24 \\
\hline H5 & 8280 & 3004 & 4442 & 26 \\
\hline H7 & 7647 & 913 & 3878 & 24 \\
\hline H9 & 8346 & 120 & 387 & 30 \\
\hline H11 & 6150 & -1142 & 734 & 34 \\
\hline H13 & 7850 & -549 & 4010 & 26 \\
\hline $\mathrm{H} 15 \mathrm{~A}$ & 9431 & 3691 & 5056 & 63 \\
\hline H15B & 10433 & 3944 & 4772 & 63 \\
\hline $\mathrm{H} 15 \mathrm{C}$ & 10325 & 3240 & 5643 & 63 \\
\hline H16A & 8880 & 3696 & 2627 & 75 \\
\hline H16B & 9450 & 3270 & 1635 & 75 \\
\hline $\mathrm{H} 16 \mathrm{C}$ & 9881 & 3973 & 2385 & 75 \\
\hline H17A & 11288 & 3289 & 3332 & 80 \\
\hline H17B & 10851 & 2621 & 2478 & 80 \\
\hline $\mathrm{H} 17 \mathrm{C}$ & 11183 & 2531 & 4037 & 80 \\
\hline H15D & 10153 & 4115 & 4378 & 84 \\
\hline $\mathrm{H} 15 \mathrm{E}$ & 9631 & 3615 & 5323 & 84 \\
\hline $\mathrm{H} 15 \mathrm{~F}$ & 9083 & 3929 & 3975 & 84 \\
\hline H16D & 10161 & 2813 & 1779 & 58 \\
\hline H16E & 10452 & 3619 & 2183 & 58 \\
\hline $\mathrm{H} 16 \mathrm{~F}$ & 9385 & 3415 & 1826 & 58 \\
\hline H17D & 11131 & 2422 & 3889 & 87 \\
\hline H17E & 10896 & 2743 & 5268 & 87 \\
\hline $\mathrm{H} 17 \mathrm{~F}$ & 11361 & 3243 & 4259 & 87 \\
\hline H19A & 6120 & 2066 & 2863 & 50 \\
\hline H19B & 5508 & 1883 & 4016 & 50 \\
\hline H19C & 6195 & 1293 & 3555 & 50 \\
\hline $\mathrm{H} 20 \mathrm{~A}$ & 6682 & 3150 & 4203 & 56 \\
\hline
\end{tabular}




\begin{tabular}{|c|c|c|c|c|}
\hline H20B & 7160 & 3055 & 5706 & 56 \\
\hline $\mathrm{H} 20 \mathrm{C}$ & 6078 & 2910 & 5333 & 56 \\
\hline $\mathrm{H} 21 \mathrm{~A}$ & 6280 & 1704 & 6354 & 50 \\
\hline $\mathrm{H} 21 \mathrm{~B}$ & 7366 & 1838 & 6711 & 50 \\
\hline $\mathrm{H} 21 \mathrm{C}$ & 6998 & 1148 & 5864 & 50 \\
\hline $\mathrm{H} 23 \mathrm{~A}$ & 7683 & 444 & -1646 & 73 \\
\hline $\mathrm{H} 23 \mathrm{~B}$ & 7480 & -83 & -2897 & 73 \\
\hline $\mathrm{H} 23 \mathrm{C}$ & 8274 & -275 & -1706 & 73 \\
\hline $\mathrm{H} 24 \mathrm{~A}$ & 5543 & -354 & -1134 & 86 \\
\hline H24B & 5857 & -121 & -2525 & 86 \\
\hline $\mathrm{H} 24 \mathrm{C}$ & 6078 & 387 & -1250 & 86 \\
\hline $\mathrm{H} 25 \mathrm{~A}$ & 6466 & -1531 & -1351 & 77 \\
\hline $\mathrm{H} 25 \mathrm{~B}$ & 7538 & -1455 & -1503 & 77 \\
\hline $\mathrm{H} 25 \mathrm{C}$ & 6769 & -1230 & -2705 & 77 \\
\hline $\mathrm{H} 23 \mathrm{D}$ & 5833 & -951 & -2573 & 70 \\
\hline $\mathrm{H} 23 \mathrm{E}$ & 5500 & -786 & -1163 & 70 \\
\hline $\mathrm{H} 23 \mathrm{~F}$ & 6135 & -1470 & -1335 & 70 \\
\hline $\mathrm{H} 24 \mathrm{D}$ & 7485 & -753 & -2826 & 81 \\
\hline $\mathrm{H} 24 \mathrm{E}$ & 7772 & -1281 & -1597 & 81 \\
\hline $\mathrm{H} 24 \mathrm{~F}$ & 8228 & -501 & -1612 & 81 \\
\hline $\mathrm{H} 25 \mathrm{D}$ & 6408 & 289 & -2503 & 66 \\
\hline $\mathrm{H} 25 \mathrm{E}$ & 7169 & 586 & -1351 & 66 \\
\hline $\mathrm{H} 25 \mathrm{~F}$ & 6148 & 412 & -1036 & 66 \\
\hline $\mathrm{H} 27 \mathrm{~A}$ & 6617 & -2090 & 4927 & 66 \\
\hline $\mathrm{H} 27 \mathrm{~B}$ & 7445 & -1532 & 4892 & 66 \\
\hline $\mathrm{H} 27 \mathrm{C}$ & 7293 & -2163 & 3816 & 66 \\
\hline $\mathrm{H} 28 \mathrm{~A}$ & 5522 & -1057 & 4860 & 62 \\
\hline $\mathrm{H} 28 \mathrm{~B}$ & 5445 & -497 & 3654 & 62 \\
\hline $\mathrm{H} 28 \mathrm{C}$ & 6335 & -493 & 4759 & 62 \\
\hline $\mathrm{H} 29 \mathrm{~A}$ & 5287 & -2085 & 3267 & 60 \\
\hline H29B & 5899 & -2140 & 2074 & 60 \\
\hline $\mathrm{H} 29 \mathrm{C}$ & 5161 & -1506 & 2090 & 60 \\
\hline
\end{tabular}


Table S6. Torsion angles $\left[{ }^{\circ}\right]$ for neijk01.

\begin{tabular}{|c|c|c|c|}
\hline C8-P1-C1-C1\#1 & $173.33(13)$ & C3-C4-C14-C16' & $68.6(5)$ \\
\hline C2-P1-C1-C1\#1 & $-81.81(14)$ & C5-C4-C14-C16' & $-108.5(5)$ \\
\hline $\mathrm{C} 8-\mathrm{P} 1-\mathrm{C} 2-\mathrm{C} 7$ & $31.62(13)$ & C3-C4-C14-C15 & $-134.5(2)$ \\
\hline $\mathrm{C} 1-\mathrm{P} 1-\mathrm{C} 2-\mathrm{C} 7$ & $-71.97(13)$ & C5-C4-C14-C15 & $48.4(3)$ \\
\hline $\mathrm{C} 8-\mathrm{P} 1-\mathrm{C} 2-\mathrm{C} 3$ & $-150.49(11)$ & C3-C4-C14-C17 & $-11.1(3)$ \\
\hline $\mathrm{C} 1-\mathrm{P} 1-\mathrm{C} 2-\mathrm{C} 3$ & 105.91(11) & C5-C4-C14-C17 & $171.7(3)$ \\
\hline $\mathrm{C} 7-\mathrm{C} 2-\mathrm{C} 3-\mathrm{C} 4$ & $0.6(2)$ & C3-C4-C14-C15' & $-166.7(6)$ \\
\hline $\mathrm{P} 1-\mathrm{C} 2-\mathrm{C} 3-\mathrm{C} 4$ & $-177.39(11)$ & C5-C4-C14-C15' & $16.2(6)$ \\
\hline $\mathrm{C} 2-\mathrm{C} 3-\mathrm{C} 4-\mathrm{C} 5$ & $-0.2(2)$ & $\mathrm{C} 3-\mathrm{C} 4-\mathrm{C} 14-\mathrm{C} 16$ & $107.7(3)$ \\
\hline $\mathrm{C} 2-\mathrm{C} 3-\mathrm{C} 4-\mathrm{C} 14$ & $-177.36(13)$ & C5-C4-C14-C16 & $-69.4(3)$ \\
\hline C3-C4-C5-C6 & $-0.1(2)$ & C3-C4-C14-C17' & $-50.7(6)$ \\
\hline C14-C4-C5-C6 & $177.09(13)$ & C5-C4-C14-C17' & $132.2(6)$ \\
\hline $\mathrm{C} 4-\mathrm{C} 5-\mathrm{C} 6-\mathrm{C} 7$ & $0.0(2)$ & C5-C6-C18-C20 & $6.1(2)$ \\
\hline C4-C5-C6-C18 & $178.56(13)$ & C7-C6-C18-C20 & $-175.37(14)$ \\
\hline $\mathrm{C} 3-\mathrm{C} 2-\mathrm{C} 7-\mathrm{C} 6$ & $-0.7(2)$ & C5-C6-C18-C19 & $126.32(15)$ \\
\hline P1-C2-C7-C6 & $177.12(11)$ & C7-C6-C18-C19 & $-55.13(18)$ \\
\hline $\mathrm{C} 5-\mathrm{C} 6-\mathrm{C} 7-\mathrm{C} 2$ & $0.4(2)$ & $\mathrm{C} 5-\mathrm{C} 6-\mathrm{C} 18-\mathrm{C} 21$ & $-114.30(16)$ \\
\hline C18-C6-C7-C2 & $-178.18(13)$ & C7-C6-C18-C21 & $64.25(18)$ \\
\hline C2-P1-C8-C13 & $-88.98(13)$ & C9-C10-C22-C24' & $59.3(3)$ \\
\hline C1-P1-C8-C13 & $13.12(14)$ & C11-C10-C22-C24' & $-119.9(3)$ \\
\hline C2-P1-C8-C9 & $95.61(12)$ & C9-C10-C22-C25' & $-67.6(3)$ \\
\hline C1-P1-C8-C9 & $-162.29(12)$ & C11-C10-C22-C25' & $113.2(3)$ \\
\hline $\mathrm{C} 13-\mathrm{C} 8-\mathrm{C} 9-\mathrm{C} 10$ & $0.3(2)$ & C9-C10-C22-C24 & $-114.9(2)$ \\
\hline P1-C8-C9-C10 & $175.92(12)$ & C11-C10-C22-C24 & $65.9(3)$ \\
\hline C8-C9-C10-C11 & $-0.8(2)$ & C9-C10-C22-C25 & $119.6(2)$ \\
\hline C8-C9-C10-C22 & 179.99(15) & $\mathrm{C} 11-\mathrm{C} 10-\mathrm{C} 22-\mathrm{C} 25$ & $-59.6(3)$ \\
\hline C9-C10-C11-C12 & $0.5(2)$ & $\mathrm{C} 9-\mathrm{C} 10-\mathrm{C} 22-\mathrm{C} 23$ & $3.4(3)$ \\
\hline $\mathrm{C} 22-\mathrm{C} 10-\mathrm{C} 11-\mathrm{C} 12$ & $179.77(15)$ & $\mathrm{C} 11-\mathrm{C} 10-\mathrm{C} 22-\mathrm{C} 23$ & $-175.8(2)$ \\
\hline $\mathrm{C} 10-\mathrm{C} 11-\mathrm{C} 12-\mathrm{C} 13$ & $0.2(2)$ & C9-C10-C22-C23' & $178.5(3)$ \\
\hline $\mathrm{C} 10-\mathrm{C} 11-\mathrm{C} 12-\mathrm{C} 26$ & $179.72(15)$ & C11-C10-C22-C23' & $-0.7(3)$ \\
\hline C9-C8-C13-C12 & $0.6(2)$ & $\mathrm{C} 11-\mathrm{C} 12-\mathrm{C} 26-\mathrm{C} 29$ & $9.2(2)$ \\
\hline $\mathrm{P} 1-\mathrm{C} 8-\mathrm{C} 13-\mathrm{C} 12$ & $-174.81(12)$ & $\mathrm{C} 13-\mathrm{C} 12-\mathrm{C} 26-\mathrm{C} 29$ & $-171.28(15)$ \\
\hline $\mathrm{C} 11-\mathrm{C} 12-\mathrm{C} 13-\mathrm{C} 8$ & $-0.8(2)$ & $\mathrm{C} 11-\mathrm{C} 12-\mathrm{C} 26-\mathrm{C} 27$ & $129.19(17)$ \\
\hline $\mathrm{C} 26-\mathrm{C} 12-\mathrm{C} 13-\mathrm{C} 8$ & 179.70(14) & $\mathrm{C} 13-\mathrm{C} 12-\mathrm{C} 26-\mathrm{C} 27$ & $-51.3(2)$ \\
\hline
\end{tabular}


Symmetry transformations used to generate equivalent atoms:

\#1 - $\mathrm{x}+2,-\mathrm{y},-\mathrm{z}+1$ 


\section{2 $\mathrm{FeCl}_{2}\left({ }^{\mathrm{tBu}} \mathrm{dppe}\right)$}

REFERENCE NUMBER: neijk04

CRYSTAL STRUCTURE REPORT

$\mathrm{C}_{66} \mathrm{H}_{104} \mathrm{Cl}_{2} \mathrm{Fe} \mathrm{O}_{4} \mathrm{P}_{2}$

or

$\mathrm{FeCl}_{2}(\mathrm{PP}) \cdot 2$ dioxane

Report prepared for:

J. Kneebone, Prof. M. Neidig

February 11, 2013

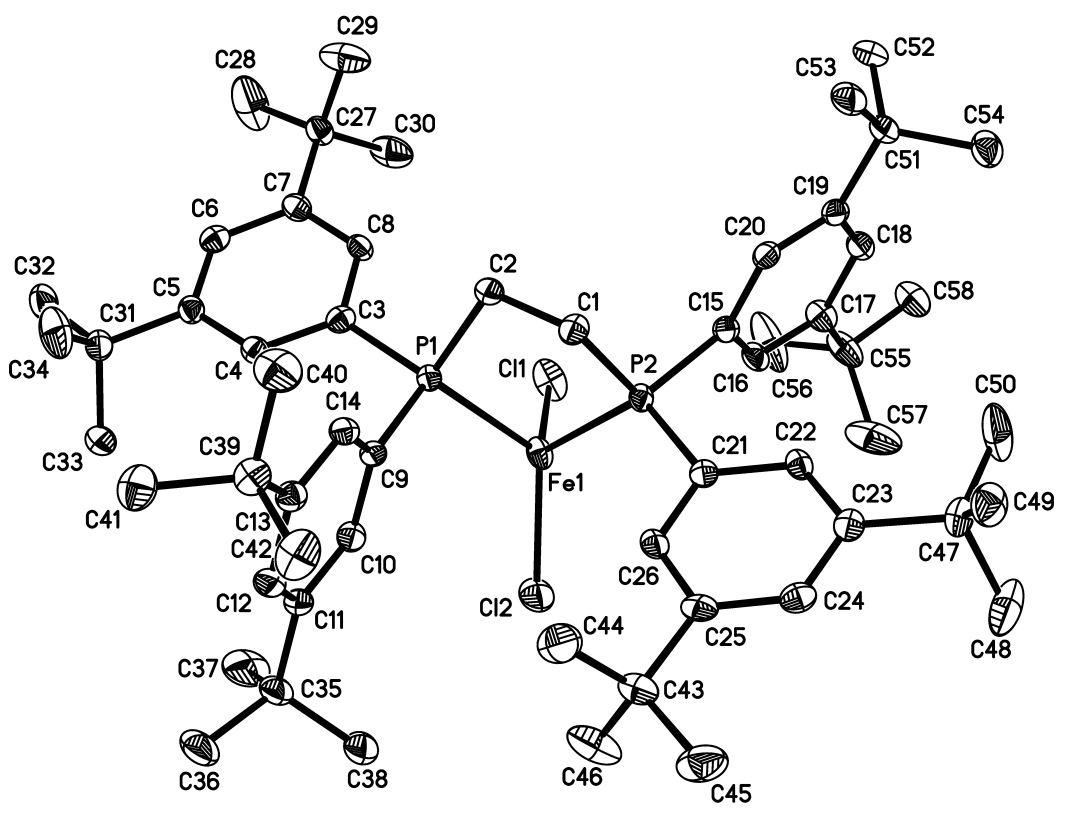

William W. Brennessel

X-ray Crystallographic Facility

Department of Chemistry, University of Rochester

120 Trustee Road

Rochester, NY 14627 


\section{Data collection}

A crystal $\left(0.48 \times 0.16 \times 0.12 \mathrm{~mm}^{3}\right)$ was placed onto the tip of a $0.1 \mathrm{~mm}$ diameter glass capillary tube or fiber and mounted on a Bruker SMART APEX II CCD Platform diffractometer for a data collection at 100.0(5) K. ${ }^{1}$ A preliminary set of cell constants and an orientation matrix were calculated from reflections harvested from three orthogonal wedges of reciprocal space. The full data collection was carried out using MoK $\alpha$ radiation (graphite monochromator) with a frame time of 90 seconds and a detector distance of $4.01 \mathrm{~cm}$. A randomly oriented region of reciprocal space was surveyed: three major sections of frames were collected with $0.50^{\circ}$ steps in $\omega$ at three different $\phi$ settings and a detector position of $-38^{\circ}$ in $2 \theta$. The intensity data were corrected for absorption. ${ }^{2}$ Final cell constants were calculated from the xyz centroids of 4038 strong reflections from the actual data collection after integration. ${ }^{3}$ See Table 1 for additional crystal and refinement information.

\section{Structure solution and refinement}

The structure was solved using SIR9 $7^{4}$ and refined using SHELXL-2012. ${ }^{5}$ The space group $P 2{ }_{1} / c$ was determined based on systematic absences. A direct-methods solution was calculated which provided most non-hydrogen atoms from the E-map. Full-matrix least squares / difference Fourier cycles were performed which located the remaining non-hydrogen atoms. All non-hydrogen atoms were refined with anisotropic displacement parameters. All hydrogen atoms were placed in ideal positions and refined as riding atoms with relative isotropic displacement parameters. The final full matrix least squares refinement converged to $R 1=0.0618\left(F^{2}, I>2 \sigma(I)\right)$ and $w R 2=$ $0.1649\left(F^{2}\right.$, all data).

\section{Structure description}

The structure is the one suggested, with all atoms in general positions. There are two cocrystallized dioxane solvent molecules per iron molecule. One tert-butyl group is modeled as disordered over two positions (89:11).

Unless noted otherwise all structural diagrams containing thermal displacement ellipsoids are drawn at the $50 \%$ probability level.

Data collection, structure solution, and structure refinement were conducted at the X-ray Crystallographic Facility, B51 Hutchison Hall, Department of Chemistry, University of Rochester. All publications arising from this report MUST either 1) include William W. Brennessel as a coauthor or 2) acknowledge William W. Brennessel and the Xray Crystallographic Facility of the Department of Chemistry at the University of Rochester. 
1 APEX2, version 2012.4-3; Bruker AXS: Madison, WI, 2012.

2 Sheldrick, G. M. SADABS, version 2008/1; University of Göttingen: Göttingen, Germany, 2008.

3 SAINT, version 7.68A; Bruker AXS: Madison, WI, 2009.

4 Altomare, A.; Burla, M. C.; Camalli, M.; Cascarano, G. L.; Giacovazzo, C.; Guagliardi, A.; Moliterni, A. G. G.;

Polidori, G.; Spagna, R. SIR97: A new program for solving and refining crystal structures; Istituto di Cristallografia, CNR: Bari, Italy, 1999.

5 Sheldrick, G. M. SHELXL-2012 University of Göttingen: Göttingen, Germany, 2012.

Some equations of interest:

$$
\begin{gathered}
R_{\mathrm{int}}=\Sigma\left|F_{\mathrm{o}}{ }^{2}-<F_{\mathrm{o}}{ }^{2}>\right| / \Sigma\left|F_{\mathrm{o}}{ }^{2}\right| \\
R 1=\Sigma|| F_{\mathrm{o}}|-| F_{\mathrm{c}} \| / \Sigma\left|F_{\mathrm{o}}\right| \\
w R 2=\left[\Sigma\left[w\left(F_{\mathrm{o}}{ }^{2}-F_{\mathrm{c}}{ }^{2}\right)^{2}\right] / \Sigma\left[w\left(F_{\mathrm{o}}{ }^{2}\right)^{2}\right]\right]^{1 / 2} \\
\text { where } w=1 /\left[\sigma^{2}\left(F_{\mathrm{o}}{ }^{2}\right)+(a P)^{2}+b P\right] \text { and } \\
P=1 / 3 \max \left(0, F_{\mathrm{o}}{ }^{2}\right)+2 / 3 F_{\mathrm{c}}{ }^{2} \\
\text { GOF }=S=\left[\Sigma\left[w\left(F_{\mathrm{o}}{ }^{2}-F_{\mathrm{c}}{ }^{2}\right)^{2}\right] /(m-n)\right]^{1 / 2}
\end{gathered}
$$

where $m=$ number of reflections and $n=$ number of parameters

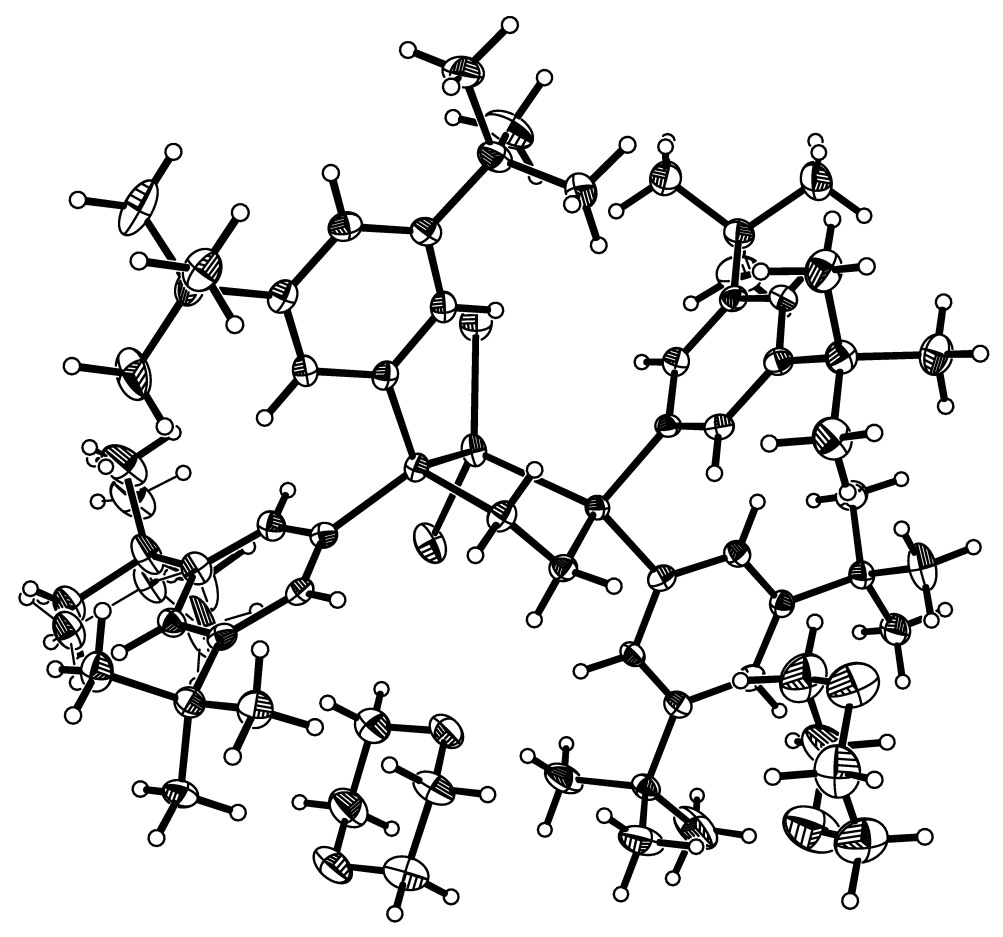


Table S7. Crystal data and structure refinement for neijk04.

\begin{tabular}{|c|c|c|}
\hline Identification code & \multicolumn{2}{|l|}{ neijk04 } \\
\hline Empirical formula & \multicolumn{2}{|c|}{$\mathrm{C} 66 \mathrm{H} 104 \mathrm{Cl} 2 \mathrm{Fe} \mathrm{O} 4 \mathrm{P} 2$} \\
\hline Formula weight & \multicolumn{2}{|l|}{1150.18} \\
\hline Temperature & \multicolumn{2}{|l|}{$100.0(5) \mathrm{K}$} \\
\hline Wavelength & \multicolumn{2}{|l|}{$0.71073 \AA$} \\
\hline Crystal system & \multicolumn{2}{|l|}{ monoclinic } \\
\hline Space group & \multicolumn{2}{|l|}{$P 2_{1} / c$} \\
\hline \multirow[t]{3}{*}{ Unit cell dimensions } & $a=14.135(4) \AA$ & $\alpha=90^{\circ}$ \\
\hline & $b=28.716(8) \AA$ & $\beta=102.479(6)^{\circ}$ \\
\hline & $c=16.870(5) \AA$ & $\gamma=90^{\circ}$ \\
\hline Volume & \multicolumn{2}{|l|}{$6686(3) \AA^{3}$} \\
\hline$Z$ & \multicolumn{2}{|l|}{4} \\
\hline Density (calculated) & \multicolumn{2}{|l|}{$1.143 \mathrm{Mg} / \mathrm{m}^{3}$} \\
\hline Absorption coefficient & \multicolumn{2}{|l|}{$0.396 \mathrm{~mm}^{-1}$} \\
\hline$F(000)$ & \multicolumn{2}{|l|}{2488} \\
\hline Crystal color, morphology & \multicolumn{2}{|l|}{ colorless, needle } \\
\hline Crystal size & \multicolumn{2}{|c|}{$0.48 \times 0.16 \times 0.12 \mathrm{~mm}^{3}$} \\
\hline Theta range for data collection & \multicolumn{2}{|l|}{1.425 to $29.574^{\circ}$} \\
\hline Index ranges & \multicolumn{2}{|c|}{$-19 \leq h \leq 19,-38 \leq k \leq 39,-23 \leq l \leq 23$} \\
\hline Reflections collected & \multicolumn{2}{|l|}{78114} \\
\hline Independent reflections & \multicolumn{2}{|c|}{$18751[R($ int $)=0.0998]$} \\
\hline Observed reflections & \multicolumn{2}{|l|}{10927} \\
\hline Completeness to theta $=29.575^{\circ}$ & \multicolumn{2}{|l|}{$99.9 \%$} \\
\hline Absorption correction & \multicolumn{2}{|l|}{ Multi-scan } \\
\hline Max. and min. transmission & \multicolumn{2}{|l|}{0.7461 and 0.6845} \\
\hline Refinement method & \multicolumn{2}{|c|}{ Full-matrix least-squares on $F^{2}$} \\
\hline Data / restraints / parameters & \multicolumn{2}{|l|}{$18751 / 7 / 713$} \\
\hline Goodness-of-fit on $F^{2}$ & \multicolumn{2}{|l|}{1.017} \\
\hline Final $R$ indices $[I>2 \operatorname{sigma}(I)]$ & \multicolumn{2}{|c|}{$R 1=0.0618, w R 2=0.1419$} \\
\hline$R$ indices (all data) & \multicolumn{2}{|c|}{$R 1=0.1193, w R 2=0.1649$} \\
\hline Largest diff. peak and hole & \multicolumn{2}{|c|}{1.687 and -0.786 e..$\AA^{-3}$} \\
\hline
\end{tabular}


Table S8. Atomic coordinates $\left(\mathrm{x} 10^{4}\right)$ and equivalent isotropic displacement parameters $\left(\AA^{2} \times 10^{3}\right)$ for neijk04. $U_{e q}$ is defined as one third of the trace of the orthogonalized $U_{i j}$ tensor.

\begin{tabular}{|c|c|c|c|c|}
\hline & $\mathrm{x}$ & $\mathrm{y}$ & $\mathrm{z}$ & $\mathrm{U}_{\mathrm{eq}}$ \\
\hline $\mathrm{Fe} 1$ & $7787(1)$ & $2417(1)$ & $5510(1)$ & $20(1)$ \\
\hline $\mathrm{Cl1}$ & 9363(1) & $2543(1)$ & $5688(1)$ & $33(1)$ \\
\hline $\mathrm{Cl} 2$ & $6910(1)$ & $2231(1)$ & $4293(1)$ & $33(1)$ \\
\hline $\mathrm{P} 1$ & $7192(1)$ & $3029(1)$ & $6269(1)$ & $17(1)$ \\
\hline $\mathrm{P} 2$ & $7415(1)$ & 1921(1) & $6587(1)$ & $16(1)$ \\
\hline $\mathrm{C} 1$ & $6956(2)$ & $2306(1)$ & $7294(2)$ & $20(1)$ \\
\hline $\mathrm{C} 2$ & $7347(2)$ & 2801(1) & $7294(2)$ & $19(1)$ \\
\hline $\mathrm{C} 3$ & $7724(2)$ & $3607(1)$ & $6414(2)$ & $19(1)$ \\
\hline $\mathrm{C} 4$ & $7223(2)$ & 3993(1) & $6031(2)$ & $19(1)$ \\
\hline $\mathrm{C} 5$ & $7646(2)$ & $4433(1)$ & $6099(2)$ & $19(1)$ \\
\hline C6 & $8591(2)$ & $4477(1)$ & $6565(2)$ & $20(1)$ \\
\hline $\mathrm{C} 7$ & $9098(2)$ & 4098(1) & $6966(2)$ & $20(1)$ \\
\hline $\mathrm{C} 8$ & $8657(2)$ & $3660(1)$ & $6881(2)$ & $18(1)$ \\
\hline $\mathrm{C} 9$ & $5911(2)$ & $3136(1)$ & $5867(2)$ & $17(1)$ \\
\hline $\mathrm{C} 10$ & $5634(2)$ & $3160(1)$ & $5026(2)$ & $20(1)$ \\
\hline $\mathrm{C} 11$ & $4692(2)$ & $3282(1)$ & $4636(2)$ & $20(1)$ \\
\hline $\mathrm{C} 12$ & $4040(2)$ & $3362(1)$ & $5125(2)$ & $21(1)$ \\
\hline $\mathrm{C} 13$ & $4288(2)$ & $3336(1)$ & $5975(2)$ & $19(1)$ \\
\hline $\mathrm{C} 14$ & $5236(2)$ & $3225(1)$ & $6340(2)$ & $17(1)$ \\
\hline $\mathrm{C} 15$ & $8459(2)$ & $1605(1)$ & $7141(2)$ & $17(1)$ \\
\hline $\mathrm{C} 16$ & $9095(2)$ & $1436(1)$ & $6689(2)$ & $20(1)$ \\
\hline $\mathrm{C} 17$ & $9877(2)$ & $1155(1)$ & $7034(2)$ & $23(1)$ \\
\hline $\mathrm{C} 18$ & $9998(2)$ & $1048(1)$ & $7854(2)$ & $21(1)$ \\
\hline C19 & $9375(2)$ & $1214(1)$ & $8332(2)$ & $19(1)$ \\
\hline $\mathrm{C} 20$ & $8609(2)$ & 1493(1) & $7966(2)$ & $18(1)$ \\
\hline $\mathrm{C} 21$ & $6468(2)$ & $1484(1)$ & $6379(2)$ & $17(1)$ \\
\hline $\mathrm{C} 22$ & $6596(2)$ & 1033(1) & $6691(2)$ & $17(1)$ \\
\hline $\mathrm{C} 23$ & $5838(2)$ & $715(1)$ & $6546(2)$ & $19(1)$ \\
\hline $\mathrm{C} 24$ & $4942(2)$ & $860(1)$ & $6082(2)$ & $21(1)$ \\
\hline $\mathrm{C} 25$ & $4796(2)$ & 1304(1) & $5763(2)$ & $20(1)$ \\
\hline $\mathrm{C} 26$ & $5573(2)$ & $1615(1)$ & $5912(2)$ & $19(1)$ \\
\hline
\end{tabular}




\begin{tabular}{|c|c|c|c|c|}
\hline $\mathrm{C} 27$ & $10110(2)$ & $4146(1)$ & $7516(2)$ & $22(1)$ \\
\hline $\mathrm{C} 28$ & $10538(3)$ & $4627(1)$ & $7474(3)$ & $67(1)$ \\
\hline $\mathrm{C} 29$ & $10055(2)$ & $4044(1)$ & $8390(2)$ & $43(1)$ \\
\hline $\mathrm{C} 30$ & $10794(2)$ & $3795(1)$ & $7259(2)$ & $37(1)$ \\
\hline $\mathrm{C} 31$ & $7062(2)$ & $4846(1)$ & $5671(2)$ & $23(1)$ \\
\hline $\mathrm{C} 32$ & $7659(2)$ & $5286(1)$ & $5715(2)$ & $34(1)$ \\
\hline $\mathrm{C} 33$ & 6671(2) & $4735(1)$ & $4771(2)$ & $32(1)$ \\
\hline $\mathrm{C} 34$ & $6210(2)$ & $4938(1)$ & $6073(2)$ & $41(1)$ \\
\hline $\mathrm{C} 35$ & $4422(2)$ & $3310(1)$ & $3708(2)$ & $26(1)$ \\
\hline $\mathrm{C} 36$ & $3442(3)$ & $3550(1)$ & $3401(2)$ & $43(1)$ \\
\hline $\mathrm{C} 37$ & 5194(3) & $3584(1)$ & $3392(2)$ & $45(1)$ \\
\hline $\mathrm{C} 38$ & $4366(3)$ & $2818(1)$ & $3360(2)$ & $36(1)$ \\
\hline C39 & $3514(2)$ & $3437(1)$ & $6457(2)$ & $23(1)$ \\
\hline $\mathrm{C} 40$ & $3920(2)$ & $3423(1)$ & $7370(2)$ & $35(1)$ \\
\hline $\mathrm{C} 41$ & $3096(2)$ & $3924(1)$ & $6235(2)$ & $32(1)$ \\
\hline $\mathrm{C} 42$ & 2694(2) & $3075(1)$ & $6242(2)$ & $35(1)$ \\
\hline $\mathrm{C} 43$ & $3821(2)$ & $1472(1)$ & $5253(2)$ & $25(1)$ \\
\hline $\mathrm{C} 44$ & $3447(2)$ & $1865(1)$ & $5698(2)$ & $43(1)$ \\
\hline $\mathrm{C} 45$ & $3061(2)$ & $1080(1)$ & $5106(2)$ & $39(1)$ \\
\hline $\mathrm{C} 46$ & $3965(3)$ & $1625(1)$ & $4427(2)$ & $47(1)$ \\
\hline $\mathrm{C} 47$ & $5956(2)$ & $216(1)$ & $6881(2)$ & $24(1)$ \\
\hline $\mathrm{C} 48$ & 5793(3) & $-127(1)$ & $6171(2)$ & $45(1)$ \\
\hline C49 & $5213(2)$ & $122(1)$ & $7400(2)$ & $31(1)$ \\
\hline $\mathrm{C} 50$ & 6963(2) & $135(1)$ & $7414(2)$ & $46(1)$ \\
\hline C51 & 9554(2) & $1078(1)$ & $9232(2)$ & $23(1)$ \\
\hline C52 & $10550(2)$ & $1260(1)$ & $9670(2)$ & $31(1)$ \\
\hline C53 & $8787(2)$ & $1280(1)$ & $9646(2)$ & $29(1)$ \\
\hline C54 & $9526(2)$ & $545(1)$ & $9304(2)$ & $28(1)$ \\
\hline C55 & $10550(4)$ & $989(2)$ & $6490(3)$ & $31(1)$ \\
\hline $\mathrm{C} 56$ & 10989(5) & $1426(2)$ & $6172(5)$ & $55(2)$ \\
\hline C57 & $9988(6)$ & $700(3)$ & $5784(3)$ & $56(2)$ \\
\hline $\mathrm{C} 58$ & $11385(4)$ & $693(2)$ & $6958(4)$ & $32(1)$ \\
\hline C55' & $10785(19)$ & $1062(13)$ & $6700(20)$ & $31(1)$ \\
\hline C56' & $11340(40)$ & $1515(13)$ & $6570(40)$ & $55(2)$ \\
\hline C57' & $10370(40)$ & $841(19)$ & $5880(20)$ & $56(2)$ \\
\hline C58' & $11540(30)$ & $735(15)$ & $7190(30)$ & $32(1)$ \\
\hline
\end{tabular}




\begin{tabular}{lllll} 
O1 & $6022(2)$ & $4123(1)$ & $8666(2)$ & $51(1)$ \\
C59 & $6532(3)$ & $4111(1)$ & $9495(2)$ & $49(1)$ \\
C60 & $7258(3)$ & $4488(2)$ & $9657(2)$ & $54(1)$ \\
O2 & $7931(2)$ & $4449(1)$ & $9153(2)$ & $67(1)$ \\
C61 & $7428(3)$ & $4455(2)$ & $8337(2)$ & $52(1)$ \\
C62 & $6698(3)$ & $4079(2)$ & $8158(2)$ & $56(1)$ \\
O3 & $9884(2)$ & $2731(1)$ & $8017(1)$ & $34(1)$ \\
C63 & $9928(2)$ & $2641(1)$ & $8855(2)$ & $34(1)$ \\
C64 & $10938(2)$ & $2722(1)$ & $9351(2)$ & $40(1)$ \\
O4 & $11630(2)$ & $2441(1)$ & $9066(2)$ & $42(1)$ \\
C65 & $11573(2)$ & $2533(1)$ & $8236(2)$ & $45(1)$ \\
C66 & $10573(2)$ & $2441(1)$ & $7748(2)$ & $42(1)$ \\
& & & & \\
\hline
\end{tabular}


Table S9. Bond lengths $[\AA]$ and angles $\left[{ }^{\circ}\right]$ for neijk04.

\begin{tabular}{|c|c|c|c|}
\hline $\mathrm{Fe}(1)-\mathrm{Cl}(1)$ & $2.2120(10)$ & $C(13)-C(14)$ & $1.386(4)$ \\
\hline $\mathrm{Fe}(1)-\mathrm{Cl}(2)$ & $2.2225(10)$ & $C(13)-C(39)$ & $1.526(4)$ \\
\hline $\mathrm{Fe}(1)-\mathrm{P}(1)$ & $2.4291(9)$ & $\mathrm{C}(14)-\mathrm{H}(14)$ & 0.9500 \\
\hline $\mathrm{Fe}(1)-\mathrm{P}(2)$ & $2.4549(9)$ & $C(15)-C(16)$ & $1.386(4)$ \\
\hline $\mathrm{P}(1)-\mathrm{C}(9)$ & $1.816(3)$ & $C(15)-C(20)$ & $1.400(4)$ \\
\hline $\mathrm{P}(1)-\mathrm{C}(3)$ & $1.818(3)$ & $C(16)-C(17)$ & $1.390(4)$ \\
\hline $\mathrm{P}(1)-\mathrm{C}(2)$ & $1.818(3)$ & $\mathrm{C}(16)-\mathrm{H}(16)$ & 0.9500 \\
\hline $\mathrm{P}(2)-\mathrm{C}(15)$ & $1.810(3)$ & $C(17)-C(18)$ & $1.390(4)$ \\
\hline $\mathrm{P}(2)-\mathrm{C}(21)$ & $1.811(3)$ & $C(17)-C(55)$ & $1.533(4)$ \\
\hline $\mathrm{P}(2)-\mathrm{C}(1)$ & $1.845(3)$ & $\mathrm{C}(17)-\mathrm{C}\left(55^{\prime}\right)$ & $1.537(10)$ \\
\hline $\mathrm{C}(1)-\mathrm{C}(2)$ & $1.526(4)$ & $C(18)-C(19)$ & $1.399(4)$ \\
\hline $\mathrm{C}(1)-\mathrm{H}(1 \mathrm{~A})$ & 0.9900 & $\mathrm{C}(18)-\mathrm{H}(18)$ & 0.9500 \\
\hline $\mathrm{C}(1)-\mathrm{H}(1 \mathrm{~B})$ & 0.9900 & $C(19)-C(20)$ & $1.382(4)$ \\
\hline $\mathrm{C}(2)-\mathrm{H}(2 \mathrm{~A})$ & 0.9900 & $C(19)-C(51)$ & $1.534(4)$ \\
\hline $\mathrm{C}(2)-\mathrm{H}(2 \mathrm{~B})$ & 0.9900 & $\mathrm{C}(20)-\mathrm{H}(20)$ & 0.9500 \\
\hline $\mathrm{C}(3)-\mathrm{C}(8)$ & $1.390(3)$ & $C(21)-C(26)$ & $1.390(4)$ \\
\hline$C(3)-C(4)$ & $1.396(4)$ & $C(21)-C(22)$ & $1.396(4)$ \\
\hline$C(4)-C(5)$ & $1.392(4)$ & $C(22)-C(23)$ & $1.388(4)$ \\
\hline $\mathrm{C}(4)-\mathrm{H}(4)$ & 0.9500 & $\mathrm{C}(22)-\mathrm{H}(22)$ & 0.9500 \\
\hline $\mathrm{C}(5)-\mathrm{C}(6)$ & $1.402(4)$ & $C(23)-C(24)$ & $1.400(4)$ \\
\hline$C(5)-C(31)$ & $1.533(4)$ & $C(23)-C(47)$ & $1.537(4)$ \\
\hline $\mathrm{C}(6)-\mathrm{C}(7)$ & $1.395(4)$ & $C(24)-C(25)$ & $1.383(4)$ \\
\hline $\mathrm{C}(6)-\mathrm{H}(6)$ & 0.9500 & $\mathrm{C}(24)-\mathrm{H}(24)$ & 0.9500 \\
\hline$C(7)-C(8)$ & $1.396(4)$ & $C(25)-C(26)$ & $1.395(4)$ \\
\hline$C(7)-C(27)$ & $1.534(4)$ & $C(25)-C(43)$ & $1.536(4)$ \\
\hline $\mathrm{C}(8)-\mathrm{H}(8)$ & 0.9500 & $\mathrm{C}(26)-\mathrm{H}(26)$ & 0.9500 \\
\hline$C(9)-C(10)$ & $1.390(4)$ & $\mathrm{C}(27)-\mathrm{C}(28)$ & $1.515(4)$ \\
\hline $\mathrm{C}(9)-\mathrm{C}(14)$ & $1.394(4)$ & $\mathrm{C}(27)-\mathrm{C}(29)$ & $1.521(4)$ \\
\hline$C(10)-C(11)$ & $1.397(4)$ & $\mathrm{C}(27)-\mathrm{C}(30)$ & $1.525(4)$ \\
\hline $\mathrm{C}(10)-\mathrm{H}(10)$ & 0.9500 & $\mathrm{C}(28)-\mathrm{H}(28 \mathrm{~A})$ & 0.9800 \\
\hline $\mathrm{C}(11)-\mathrm{C}(12)$ & $1.382(4)$ & $\mathrm{C}(28)-\mathrm{H}(28 \mathrm{~B})$ & 0.9800 \\
\hline$C(11)-C(35)$ & $1.531(4)$ & $\mathrm{C}(28)-\mathrm{H}(28 \mathrm{C})$ & 0.9800 \\
\hline$C(12)-C(13)$ & $1.402(4)$ & $\mathrm{C}(29)-\mathrm{H}(29 \mathrm{~A})$ & 0.9800 \\
\hline $\mathrm{C}(12)-\mathrm{H}(12)$ & 0.9500 & $\mathrm{C}(29)-\mathrm{H}(29 \mathrm{~B})$ & 0.9800 \\
\hline
\end{tabular}




\begin{tabular}{|c|c|c|c|}
\hline C(29)-H(29C) & 0.9800 & $\mathrm{C}(41)-\mathrm{H}(41 \mathrm{C})$ & 0.9800 \\
\hline $\mathrm{C}(30)-\mathrm{H}(30 \mathrm{~A})$ & 0.9800 & $\mathrm{C}(42)-\mathrm{H}(42 \mathrm{~A})$ & 0.9800 \\
\hline $\mathrm{C}(30)-\mathrm{H}(30 \mathrm{~B})$ & 0.9800 & $\mathrm{C}(42)-\mathrm{H}(42 \mathrm{~B})$ & 0.9800 \\
\hline $\mathrm{C}(30)-\mathrm{H}(30 \mathrm{C})$ & 0.9800 & $\mathrm{C}(42)-\mathrm{H}(42 \mathrm{C})$ & 0.9800 \\
\hline$C(31)-C(32)$ & $1.513(4)$ & $C(43)-C(44)$ & $1.513(4)$ \\
\hline$C(31)-C(34)$ & $1.527(4)$ & $C(43)-C(46)$ & $1.517(4)$ \\
\hline $\mathrm{C}(31)-\mathrm{C}(33)$ & $1.534(4)$ & $C(43)-C(45)$ & $1.540(4)$ \\
\hline $\mathrm{C}(32)-\mathrm{H}(32 \mathrm{~A})$ & 0.9800 & $\mathrm{C}(44)-\mathrm{H}(44 \mathrm{~A})$ & 0.9800 \\
\hline $\mathrm{C}(32)-\mathrm{H}(32 \mathrm{~B})$ & 0.9800 & $\mathrm{C}(44)-\mathrm{H}(44 \mathrm{~B})$ & 0.9800 \\
\hline $\mathrm{C}(32)-\mathrm{H}(32 \mathrm{C})$ & 0.9800 & $\mathrm{C}(44)-\mathrm{H}(44 \mathrm{C})$ & 0.9800 \\
\hline $\mathrm{C}(33)-\mathrm{H}(33 \mathrm{~A})$ & 0.9800 & $\mathrm{C}(45)-\mathrm{H}(45 \mathrm{~A})$ & 0.9800 \\
\hline $\mathrm{C}(33)-\mathrm{H}(33 \mathrm{~B})$ & 0.9800 & $\mathrm{C}(45)-\mathrm{H}(45 \mathrm{~B})$ & 0.9800 \\
\hline $\mathrm{C}(33)-\mathrm{H}(33 \mathrm{C})$ & 0.9800 & $\mathrm{C}(45)-\mathrm{H}(45 \mathrm{C})$ & 0.9800 \\
\hline $\mathrm{C}(34)-\mathrm{H}(34 \mathrm{~A})$ & 0.9800 & $\mathrm{C}(46)-\mathrm{H}(46 \mathrm{~A})$ & 0.9800 \\
\hline $\mathrm{C}(34)-\mathrm{H}(34 \mathrm{~B})$ & 0.9800 & $\mathrm{C}(46)-\mathrm{H}(46 \mathrm{~B})$ & 0.9800 \\
\hline $\mathrm{C}(34)-\mathrm{H}(34 \mathrm{C})$ & 0.9800 & $\mathrm{C}(46)-\mathrm{H}(46 \mathrm{C})$ & 0.9800 \\
\hline$C(35)-C(38)$ & $1.525(4)$ & $\mathrm{C}(47)-\mathrm{C}(50)$ & $1.528(4)$ \\
\hline$C(35)-C(37)$ & $1.533(4)$ & $\mathrm{C}(47)-\mathrm{C}(48)$ & $1.530(4)$ \\
\hline$C(35)-C(36)$ & $1.534(4)$ & $C(47)-C(49)$ & $1.531(4)$ \\
\hline $\mathrm{C}(36)-\mathrm{H}(36 \mathrm{~A})$ & 0.9800 & $\mathrm{C}(48)-\mathrm{H}(48 \mathrm{~A})$ & 0.9800 \\
\hline $\mathrm{C}(36)-\mathrm{H}(36 \mathrm{~B})$ & 0.9800 & $\mathrm{C}(48)-\mathrm{H}(48 \mathrm{~B})$ & 0.9800 \\
\hline $\mathrm{C}(36)-\mathrm{H}(36 \mathrm{C})$ & 0.9800 & $\mathrm{C}(48)-\mathrm{H}(48 \mathrm{C})$ & 0.9800 \\
\hline $\mathrm{C}(37)-\mathrm{H}(37 \mathrm{~A})$ & 0.9800 & $\mathrm{C}(49)-\mathrm{H}(49 \mathrm{~A})$ & 0.9800 \\
\hline $\mathrm{C}(37)-\mathrm{H}(37 \mathrm{~B})$ & 0.9800 & $\mathrm{C}(49)-\mathrm{H}(49 \mathrm{~B})$ & 0.9800 \\
\hline $\mathrm{C}(37)-\mathrm{H}(37 \mathrm{C})$ & 0.9800 & $\mathrm{C}(49)-\mathrm{H}(49 \mathrm{C})$ & 0.9800 \\
\hline $\mathrm{C}(38)-\mathrm{H}(38 \mathrm{~A})$ & 0.9800 & $\mathrm{C}(50)-\mathrm{H}(50 \mathrm{~A})$ & 0.9800 \\
\hline $\mathrm{C}(38)-\mathrm{H}(38 \mathrm{~B})$ & 0.9800 & $\mathrm{C}(50)-\mathrm{H}(50 \mathrm{~B})$ & 0.9800 \\
\hline $\mathrm{C}(38)-\mathrm{H}(38 \mathrm{C})$ & 0.9800 & $\mathrm{C}(50)-\mathrm{H}(50 \mathrm{C})$ & 0.9800 \\
\hline$C(39)-C(40)$ & $1.523(4)$ & $\mathrm{C}(51)-\mathrm{C}(53)$ & $1.525(4)$ \\
\hline$C(39)-C(41)$ & $1.534(4)$ & $\mathrm{C}(51)-\mathrm{C}(52)$ & $1.533(4)$ \\
\hline$C(39)-C(42)$ & $1.541(4)$ & $\mathrm{C}(51)-\mathrm{C}(54)$ & $1.537(4)$ \\
\hline $\mathrm{C}(40)-\mathrm{H}(40 \mathrm{~A})$ & 0.9800 & $\mathrm{C}(52)-\mathrm{H}(52 \mathrm{~A})$ & 0.9800 \\
\hline $\mathrm{C}(40)-\mathrm{H}(40 \mathrm{~B})$ & 0.9800 & $\mathrm{C}(52)-\mathrm{H}(52 \mathrm{~B})$ & 0.9800 \\
\hline $\mathrm{C}(40)-\mathrm{H}(40 \mathrm{C})$ & 0.9800 & $\mathrm{C}(52)-\mathrm{H}(52 \mathrm{C})$ & 0.9800 \\
\hline $\mathrm{C}(41)-\mathrm{H}(41 \mathrm{~A})$ & 0.9800 & $\mathrm{C}(53)-\mathrm{H}(53 \mathrm{~A})$ & 0.9800 \\
\hline $\mathrm{C}(41)-\mathrm{H}(41 \mathrm{~B})$ & 0.9800 & $\mathrm{C}(53)-\mathrm{H}(53 \mathrm{~B})$ & 0.9800 \\
\hline
\end{tabular}




\begin{tabular}{|c|c|c|c|}
\hline $\mathrm{C}(53)-\mathrm{H}(53 \mathrm{C})$ & 0.9800 & $\mathrm{O}(2)-\mathrm{C}(61)$ & $1.407(4)$ \\
\hline $\mathrm{C}(54)-\mathrm{H}(54 \mathrm{~A})$ & 0.9800 & $C(61)-C(62)$ & $1.479(6)$ \\
\hline $\mathrm{C}(54)-\mathrm{H}(54 \mathrm{~B})$ & 0.9800 & $\mathrm{C}(61)-\mathrm{H}(61 \mathrm{~A})$ & 0.9900 \\
\hline $\mathrm{C}(54)-\mathrm{H}(54 \mathrm{C})$ & 0.9800 & $\mathrm{C}(61)-\mathrm{H}(61 \mathrm{~B})$ & 0.9900 \\
\hline$C(55)-C(57)$ & $1.527(6)$ & $\mathrm{C}(62)-\mathrm{H}(62 \mathrm{~A})$ & 0.9900 \\
\hline$C(55)-C(58)$ & $1.529(5)$ & $\mathrm{C}(62)-\mathrm{H}(62 \mathrm{~B})$ & 0.9900 \\
\hline$C(55)-C(56)$ & $1.546(5)$ & $\mathrm{O}(3)-\mathrm{C}(63)$ & $1.425(4)$ \\
\hline $\mathrm{C}(56)-\mathrm{H}(56 \mathrm{~A})$ & 0.9800 & $\mathrm{O}(3)-\mathrm{C}(66)$ & $1.428(4)$ \\
\hline $\mathrm{C}(56)-\mathrm{H}(56 \mathrm{~B})$ & 0.9800 & $C(63)-C(64)$ & $1.508(4)$ \\
\hline $\mathrm{C}(56)-\mathrm{H}(56 \mathrm{C})$ & 0.9800 & $\mathrm{C}(63)-\mathrm{H}(63 \mathrm{~A})$ & 0.9900 \\
\hline $\mathrm{C}(57)-\mathrm{H}(57 \mathrm{~A})$ & 0.9800 & $\mathrm{C}(63)-\mathrm{H}(63 \mathrm{~B})$ & 0.9900 \\
\hline $\mathrm{C}(57)-\mathrm{H}(57 \mathrm{~B})$ & 0.9800 & $\mathrm{C}(64)-\mathrm{O}(4)$ & $1.430(4)$ \\
\hline $\mathrm{C}(57)-\mathrm{H}(57 \mathrm{C})$ & 0.9800 & $\mathrm{C}(64)-\mathrm{H}(64 \mathrm{~A})$ & 0.9900 \\
\hline $\mathrm{C}(58)-\mathrm{H}(58 \mathrm{~A})$ & 0.9800 & $\mathrm{C}(64)-\mathrm{H}(64 \mathrm{~B})$ & 0.9900 \\
\hline $\mathrm{C}(58)-\mathrm{H}(58 \mathrm{~B})$ & 0.9800 & $\mathrm{O}(4)-\mathrm{C}(65)$ & $1.410(4)$ \\
\hline $\mathrm{C}(58)-\mathrm{H}(58 \mathrm{C})$ & 0.9800 & $C(65)-C(66)$ & $1.498(5)$ \\
\hline $\mathrm{C}\left(55^{\prime}\right)-\mathrm{C}\left(57^{\prime}\right)$ & $1.509(19)$ & $\mathrm{C}(65)-\mathrm{H}(65 \mathrm{~A})$ & 0.9900 \\
\hline $\mathrm{C}\left(55^{\prime}\right)-\mathrm{C}\left(58^{\prime}\right)$ & $1.532(18)$ & $\mathrm{C}(65)-\mathrm{H}(65 \mathrm{~B})$ & 0.9900 \\
\hline $\mathrm{C}\left(55^{\prime}\right)-\mathrm{C}\left(56^{\prime}\right)$ & $1.555(19)$ & $\mathrm{C}(66)-\mathrm{H}(66 \mathrm{~A})$ & 0.9900 \\
\hline $\mathrm{C}\left(56^{\prime}\right)-\mathrm{H}(56 \mathrm{D})$ & 0.9800 & $\mathrm{C}(66)-\mathrm{H}(66 \mathrm{~B})$ & 0.9900 \\
\hline $\mathrm{C}\left(56^{\prime}\right)-\mathrm{H}(56 \mathrm{E})$ & 0.9800 & $\mathrm{Cl}(1)-\mathrm{Fe}(1)-\mathrm{Cl}(2)$ & $120.65(4)$ \\
\hline $\mathrm{C}\left(56^{\prime}\right)-\mathrm{H}(56 \mathrm{~F})$ & 0.9800 & $\mathrm{Cl}(1)-\mathrm{Fe}(1)-\mathrm{P}(1)$ & $105.28(3)$ \\
\hline $\mathrm{C}\left(57^{\prime}\right)-\mathrm{H}(57 \mathrm{D})$ & 0.9800 & $\mathrm{Cl}(2)-\mathrm{Fe}(1)-\mathrm{P}(1)$ & $118.25(3)$ \\
\hline $\mathrm{C}\left(57^{\prime}\right)-\mathrm{H}(57 \mathrm{E})$ & 0.9800 & $\mathrm{Cl}(1)-\mathrm{Fe}(1)-\mathrm{P}(2)$ & $111.39(3)$ \\
\hline $\mathrm{C}\left(57^{\prime}\right)-\mathrm{H}(57 \mathrm{~F})$ & 0.9800 & $\mathrm{Cl}(2)-\mathrm{Fe}(1)-\mathrm{P}(2)$ & $112.20(4)$ \\
\hline $\mathrm{C}\left(58^{\prime}\right)-\mathrm{H}(58 \mathrm{D})$ & 0.9800 & $\mathrm{P}(1)-\mathrm{Fe}(1)-\mathrm{P}(2)$ & $82.85(3)$ \\
\hline $\mathrm{C}\left(58^{\prime}\right)-\mathrm{H}(58 \mathrm{E})$ & 0.9800 & $\mathrm{C}(9)-\mathrm{P}(1)-\mathrm{C}(3)$ & $104.28(12)$ \\
\hline $\mathrm{C}\left(58^{\prime}\right)-\mathrm{H}(58 \mathrm{~F})$ & 0.9800 & $\mathrm{C}(9)-\mathrm{P}(1)-\mathrm{C}(2)$ & $108.75(12)$ \\
\hline $\mathrm{O}(1)-\mathrm{C}(62)$ & $1.419(4)$ & $\mathrm{C}(3)-\mathrm{P}(1)-\mathrm{C}(2)$ & $103.83(12)$ \\
\hline $\mathrm{O}(1)-\mathrm{C}(59)$ & $1.429(4)$ & $\mathrm{C}(9)-\mathrm{P}(1)-\mathrm{Fe}(1)$ & $110.86(9)$ \\
\hline$C(59)-C(60)$ & $1.475(5)$ & $\mathrm{C}(3)-\mathrm{P}(1)-\mathrm{Fe}(1)$ & $123.44(9)$ \\
\hline C(59)-H(59A) & 0.9900 & $\mathrm{C}(2)-\mathrm{P}(1)-\mathrm{Fe}(1)$ & $104.90(9)$ \\
\hline C(59)-H(59B) & 0.9900 & $\mathrm{C}(15)-\mathrm{P}(2)-\mathrm{C}(21)$ & $103.85(12)$ \\
\hline $\mathrm{C}(60)-\mathrm{O}(2)$ & $1.410(4)$ & $\mathrm{C}(15)-\mathrm{P}(2)-\mathrm{C}(1)$ & $109.18(13)$ \\
\hline $\mathrm{C}(60)-\mathrm{H}(60 \mathrm{~A})$ & 0.9900 & $\mathrm{C}(21)-\mathrm{P}(2)-\mathrm{C}(1)$ & $101.05(12)$ \\
\hline $\mathrm{C}(60)-\mathrm{H}(60 \mathrm{~B})$ & 0.9900 & $\mathrm{C}(15)-\mathrm{P}(2)-\mathrm{Fe}(1)$ & $113.05(9)$ \\
\hline
\end{tabular}




\begin{tabular}{|c|c|c|c|}
\hline $\mathrm{C}(21)-\mathrm{P}(2)-\mathrm{Fe}(1)$ & $121.71(9)$ & $\mathrm{C}(9)-\mathrm{C}(10)-\mathrm{H}(10)$ & 119.3 \\
\hline $\mathrm{C}(1)-\mathrm{P}(2)-\mathrm{Fe}(1)$ & $107.00(9)$ & $\mathrm{C}(11)-\mathrm{C}(10)-\mathrm{H}(10)$ & 119.3 \\
\hline $\mathrm{C}(2)-\mathrm{C}(1)-\mathrm{P}(2)$ & $112.36(18)$ & $\mathrm{C}(12)-\mathrm{C}(11)-\mathrm{C}(10)$ & $116.9(2)$ \\
\hline $\mathrm{C}(2)-\mathrm{C}(1)-\mathrm{H}(1 \mathrm{~A})$ & 109.1 & $\mathrm{C}(12)-\mathrm{C}(11)-\mathrm{C}(35)$ & $123.4(2)$ \\
\hline $\mathrm{P}(2)-\mathrm{C}(1)-\mathrm{H}(1 \mathrm{~A})$ & 109.1 & $\mathrm{C}(10)-\mathrm{C}(11)-\mathrm{C}(35)$ & 119.7(2) \\
\hline $\mathrm{C}(2)-\mathrm{C}(1)-\mathrm{H}(1 \mathrm{~B})$ & 109.1 & $\mathrm{C}(11)-\mathrm{C}(12)-\mathrm{C}(13)$ & $123.3(2)$ \\
\hline $\mathrm{P}(2)-\mathrm{C}(1)-\mathrm{H}(1 \mathrm{~B})$ & 109.1 & $\mathrm{C}(11)-\mathrm{C}(12)-\mathrm{H}(12)$ & 118.4 \\
\hline $\mathrm{H}(1 \mathrm{~A})-\mathrm{C}(1)-\mathrm{H}(1 \mathrm{~B})$ & 107.9 & $\mathrm{C}(13)-\mathrm{C}(12)-\mathrm{H}(12)$ & 118.4 \\
\hline $\mathrm{C}(1)-\mathrm{C}(2)-\mathrm{P}(1)$ & $111.48(18)$ & $\mathrm{C}(14)-\mathrm{C}(13)-\mathrm{C}(12)$ & $118.1(2)$ \\
\hline $\mathrm{C}(1)-\mathrm{C}(2)-\mathrm{H}(2 \mathrm{~A})$ & 109.3 & $C(14)-C(13)-C(39)$ & $122.8(2)$ \\
\hline $\mathrm{P}(1)-\mathrm{C}(2)-\mathrm{H}(2 \mathrm{~A})$ & 109.3 & $\mathrm{C}(12)-\mathrm{C}(13)-\mathrm{C}(39)$ & $119.0(2)$ \\
\hline $\mathrm{C}(1)-\mathrm{C}(2)-\mathrm{H}(2 \mathrm{~B})$ & 109.3 & $C(13)-C(14)-C(9)$ & $120.2(2)$ \\
\hline $\mathrm{P}(1)-\mathrm{C}(2)-\mathrm{H}(2 \mathrm{~B})$ & 109.3 & $\mathrm{C}(13)-\mathrm{C}(14)-\mathrm{H}(14)$ & 119.9 \\
\hline $\mathrm{H}(2 \mathrm{~A})-\mathrm{C}(2)-\mathrm{H}(2 \mathrm{~B})$ & 108.0 & $\mathrm{C}(9)-\mathrm{C}(14)-\mathrm{H}(14)$ & 119.9 \\
\hline $\mathrm{C}(8)-\mathrm{C}(3)-\mathrm{C}(4)$ & $120.0(2)$ & $C(16)-C(15)-C(20)$ & 119.3(2) \\
\hline $\mathrm{C}(8)-\mathrm{C}(3)-\mathrm{P}(1)$ & $119.4(2)$ & $\mathrm{C}(16)-\mathrm{C}(15)-\mathrm{P}(2)$ & $116.5(2)$ \\
\hline $\mathrm{C}(4)-\mathrm{C}(3)-\mathrm{P}(1)$ & $120.6(2)$ & $\mathrm{C}(20)-\mathrm{C}(15)-\mathrm{P}(2)$ & $124.0(2)$ \\
\hline$C(5)-C(4)-C(3)$ & 121.1(2) & $C(15)-C(16)-C(17)$ & 121.6(3) \\
\hline $\mathrm{C}(5)-\mathrm{C}(4)-\mathrm{H}(4)$ & 119.5 & $\mathrm{C}(15)-\mathrm{C}(16)-\mathrm{H}(16)$ & 119.2 \\
\hline $\mathrm{C}(3)-\mathrm{C}(4)-\mathrm{H}(4)$ & 119.5 & $\mathrm{C}(17)-\mathrm{C}(16)-\mathrm{H}(16)$ & 119.2 \\
\hline$C(4)-C(5)-C(6)$ & $117.9(2)$ & $C(16)-C(17)-C(18)$ & $117.4(2)$ \\
\hline $\mathrm{C}(4)-\mathrm{C}(5)-\mathrm{C}(31)$ & $119.0(2)$ & $\mathrm{C}(16)-\mathrm{C}(17)-\mathrm{C}(55)$ & $118.0(3)$ \\
\hline $\mathrm{C}(6)-\mathrm{C}(5)-\mathrm{C}(31)$ & $123.1(2)$ & $\mathrm{C}(18)-\mathrm{C}(17)-\mathrm{C}(55)$ & $124.6(3)$ \\
\hline$C(7)-C(6)-C(5)$ & $122.0(2)$ & $\mathrm{C}(16)-\mathrm{C}(17)-\mathrm{C}\left(55^{\prime}\right)$ & $126.7(13)$ \\
\hline $\mathrm{C}(7)-\mathrm{C}(6)-\mathrm{H}(6)$ & 119.0 & $\mathrm{C}(18)-\mathrm{C}(17)-\mathrm{C}\left(55^{\prime}\right)$ & $113.5(15)$ \\
\hline $\mathrm{C}(5)-\mathrm{C}(6)-\mathrm{H}(6)$ & 119.0 & $\mathrm{C}(17)-\mathrm{C}(18)-\mathrm{C}(19)$ & $122.8(2)$ \\
\hline$C(6)-C(7)-C(8)$ & $118.8(2)$ & $\mathrm{C}(17)-\mathrm{C}(18)-\mathrm{H}(18)$ & 118.6 \\
\hline $\mathrm{C}(6)-\mathrm{C}(7)-\mathrm{C}(27)$ & $122.4(2)$ & $\mathrm{C}(19)-\mathrm{C}(18)-\mathrm{H}(18)$ & 118.6 \\
\hline $\mathrm{C}(8)-\mathrm{C}(7)-\mathrm{C}(27)$ & $118.8(2)$ & $\mathrm{C}(20)-\mathrm{C}(19)-\mathrm{C}(18)$ & $118.0(2)$ \\
\hline $\mathrm{C}(3)-\mathrm{C}(8)-\mathrm{C}(7)$ & $120.3(2)$ & $\mathrm{C}(20)-\mathrm{C}(19)-\mathrm{C}(51)$ & $122.3(2)$ \\
\hline $\mathrm{C}(3)-\mathrm{C}(8)-\mathrm{H}(8)$ & 119.9 & $\mathrm{C}(18)-\mathrm{C}(19)-\mathrm{C}(51)$ & $119.7(2)$ \\
\hline $\mathrm{C}(7)-\mathrm{C}(8)-\mathrm{H}(8)$ & 119.9 & $\mathrm{C}(19)-\mathrm{C}(20)-\mathrm{C}(15)$ & $120.9(2)$ \\
\hline $\mathrm{C}(10)-\mathrm{C}(9)-\mathrm{C}(14)$ & $119.9(2)$ & $\mathrm{C}(19)-\mathrm{C}(20)-\mathrm{H}(20)$ & 119.5 \\
\hline $\mathrm{C}(10)-\mathrm{C}(9)-\mathrm{P}(1)$ & $115.3(2)$ & $\mathrm{C}(15)-\mathrm{C}(20)-\mathrm{H}(20)$ & 119.5 \\
\hline $\mathrm{C}(14)-\mathrm{C}(9)-\mathrm{P}(1)$ & $124.6(2)$ & $\mathrm{C}(26)-\mathrm{C}(21)-\mathrm{C}(22)$ & $119.4(2)$ \\
\hline$C(9)-C(10)-C(11)$ & $121.5(3)$ & $\mathrm{C}(26)-\mathrm{C}(21)-\mathrm{P}(2)$ & $117.9(2)$ \\
\hline
\end{tabular}




\begin{tabular}{|c|c|}
\hline$C(22)-C(21)-P(2)$ & $122.7(2)$ \\
\hline$C(23)-C(22)-C(21)$ & $120.8(2)$ \\
\hline $\mathrm{C}(23)-\mathrm{C}(22)-\mathrm{H}(22)$ & 119.6 \\
\hline $\mathrm{C}(21)-\mathrm{C}(22)-\mathrm{H}(22)$ & 119.6 \\
\hline$C(22)-C(23)-C(24)$ & $118.3(2)$ \\
\hline$C(22)-C(23)-C(47)$ & $122.0(2)$ \\
\hline $\mathrm{C}(24)-\mathrm{C}(23)-\mathrm{C}(47)$ & $119.7(2)$ \\
\hline$C(25)-C(24)-C(23)$ & $122.1(3)$ \\
\hline $\mathrm{C}(25)-\mathrm{C}(24)-\mathrm{H}(24)$ & 118.9 \\
\hline $\mathrm{C}(23)-\mathrm{C}(24)-\mathrm{H}(24)$ & 118.9 \\
\hline$C(24)-C(25)-C(26)$ & $118.3(2)$ \\
\hline$C(24)-C(25)-C(43)$ & $123.3(2)$ \\
\hline$C(26)-C(25)-C(43)$ & $118.4(2)$ \\
\hline$C(21)-C(26)-C(25)$ & $121.0(2)$ \\
\hline $\mathrm{C}(21)-\mathrm{C}(26)-\mathrm{H}(26)$ & 119.5 \\
\hline $\mathrm{C}(25)-\mathrm{C}(26)-\mathrm{H}(26)$ & 119.5 \\
\hline $\mathrm{C}(28)-\mathrm{C}(27)-\mathrm{C}(29)$ & $109.0(3)$ \\
\hline $\mathrm{C}(28)-\mathrm{C}(27)-\mathrm{C}(30)$ & $107.8(3)$ \\
\hline $\mathrm{C}(29)-\mathrm{C}(27)-\mathrm{C}(30)$ & $108.5(3)$ \\
\hline $\mathrm{C}(28)-\mathrm{C}(27)-\mathrm{C}(7)$ & $112.6(2)$ \\
\hline $\mathrm{C}(29)-\mathrm{C}(27)-\mathrm{C}(7)$ & $109.5(2)$ \\
\hline$C(30)-C(27)-C(7)$ & $109.4(2)$ \\
\hline $\mathrm{C}(27)-\mathrm{C}(28)-\mathrm{H}(28 \mathrm{~A})$ & 109.5 \\
\hline $\mathrm{C}(27)-\mathrm{C}(28)-\mathrm{H}(28 \mathrm{~B})$ & 109.5 \\
\hline $\mathrm{H}(28 \mathrm{~A})-\mathrm{C}(28)-\mathrm{H}(28 \mathrm{~B})$ & 109.5 \\
\hline $\mathrm{C}(27)-\mathrm{C}(28)-\mathrm{H}(28 \mathrm{C})$ & 109.5 \\
\hline $\mathrm{H}(28 \mathrm{~A})-\mathrm{C}(28)-\mathrm{H}(28 \mathrm{C})$ & 109.5 \\
\hline $\mathrm{H}(28 \mathrm{~B})-\mathrm{C}(28)-\mathrm{H}(28 \mathrm{C})$ & 109.5 \\
\hline $\mathrm{C}(27)-\mathrm{C}(29)-\mathrm{H}(29 \mathrm{~A})$ & 109.5 \\
\hline $\mathrm{C}(27)-\mathrm{C}(29)-\mathrm{H}(29 \mathrm{~B})$ & 109.5 \\
\hline H(29A)-C(29)-H(29B) & 109.5 \\
\hline $\mathrm{C}(27)-\mathrm{C}(29)-\mathrm{H}(29 \mathrm{C})$ & 109.5 \\
\hline $\mathrm{H}(29 \mathrm{~A})-\mathrm{C}(29)-\mathrm{H}(29 \mathrm{C})$ & 109.5 \\
\hline H(29B)-C(29)-H(29C) & 109.5 \\
\hline $\mathrm{C}(27)-\mathrm{C}(30)-\mathrm{H}(30 \mathrm{~A})$ & 109.5 \\
\hline $\mathrm{C}(27)-\mathrm{C}(30)-\mathrm{H}(30 \mathrm{~B})$ & 109.5 \\
\hline
\end{tabular}

\begin{tabular}{|c|c|}
\hline $\mathrm{H}(30 \mathrm{~A})-\mathrm{C}(30)-\mathrm{H}(30 \mathrm{~B})$ & 109.5 \\
\hline $\mathrm{C}(27)-\mathrm{C}(30)-\mathrm{H}(30 \mathrm{C})$ & 109.5 \\
\hline $\mathrm{H}(30 \mathrm{~A})-\mathrm{C}(30)-\mathrm{H}(30 \mathrm{C})$ & 109.5 \\
\hline $\mathrm{H}(30 \mathrm{~B})-\mathrm{C}(30)-\mathrm{H}(30 \mathrm{C})$ & 109.5 \\
\hline C(32)-C(31)-C(34) & $108.5(3)$ \\
\hline$C(32)-C(31)-C(5)$ & $112.6(2)$ \\
\hline$C(34)-C(31)-C(5)$ & $108.8(2)$ \\
\hline$C(32)-C(31)-C(33)$ & $107.4(2)$ \\
\hline$C(34)-C(31)-C(33)$ & $109.0(3)$ \\
\hline $\mathrm{C}(5)-\mathrm{C}(31)-\mathrm{C}(33)$ & $110.3(2)$ \\
\hline $\mathrm{C}(31)-\mathrm{C}(32)-\mathrm{H}(32 \mathrm{~A})$ & 109.5 \\
\hline $\mathrm{C}(31)-\mathrm{C}(32)-\mathrm{H}(32 \mathrm{~B})$ & 109.5 \\
\hline $\mathrm{H}(32 \mathrm{~A})-\mathrm{C}(32)-\mathrm{H}(32 \mathrm{~B})$ & 109.5 \\
\hline $\mathrm{C}(31)-\mathrm{C}(32)-\mathrm{H}(32 \mathrm{C})$ & 109.5 \\
\hline $\mathrm{H}(32 \mathrm{~A})-\mathrm{C}(32)-\mathrm{H}(32 \mathrm{C})$ & 109.5 \\
\hline $\mathrm{H}(32 \mathrm{~B})-\mathrm{C}(32)-\mathrm{H}(32 \mathrm{C})$ & 109.5 \\
\hline $\mathrm{C}(31)-\mathrm{C}(33)-\mathrm{H}(33 \mathrm{~A})$ & 109.5 \\
\hline $\mathrm{C}(31)-\mathrm{C}(33)-\mathrm{H}(33 \mathrm{~B})$ & 109.5 \\
\hline $\mathrm{H}(33 \mathrm{~A})-\mathrm{C}(33)-\mathrm{H}(33 \mathrm{~B})$ & 109.5 \\
\hline $\mathrm{C}(31)-\mathrm{C}(33)-\mathrm{H}(33 \mathrm{C})$ & 109.5 \\
\hline $\mathrm{H}(33 \mathrm{~A})-\mathrm{C}(33)-\mathrm{H}(33 \mathrm{C})$ & 109.5 \\
\hline H(33B)-C(33)-H(33C) & 109.5 \\
\hline $\mathrm{C}(31)-\mathrm{C}(34)-\mathrm{H}(34 \mathrm{~A})$ & 109.5 \\
\hline $\mathrm{C}(31)-\mathrm{C}(34)-\mathrm{H}(34 \mathrm{~B})$ & 109.5 \\
\hline $\mathrm{H}(34 \mathrm{~A})-\mathrm{C}(34)-\mathrm{H}(34 \mathrm{~B})$ & 109.5 \\
\hline $\mathrm{C}(31)-\mathrm{C}(34)-\mathrm{H}(34 \mathrm{C})$ & 109.5 \\
\hline $\mathrm{H}(34 \mathrm{~A})-\mathrm{C}(34)-\mathrm{H}(34 \mathrm{C})$ & 109.5 \\
\hline $\mathrm{H}(34 \mathrm{~B})-\mathrm{C}(34)-\mathrm{H}(34 \mathrm{C})$ & 109.5 \\
\hline $\mathrm{C}(38)-\mathrm{C}(35)-\mathrm{C}(11)$ & $109.1(2)$ \\
\hline $\mathrm{C}(38)-\mathrm{C}(35)-\mathrm{C}(37)$ & $109.0(3)$ \\
\hline $\mathrm{C}(11)-\mathrm{C}(35)-\mathrm{C}(37)$ & $110.1(2)$ \\
\hline $\mathrm{C}(38)-\mathrm{C}(35)-\mathrm{C}(36)$ & $108.6(3)$ \\
\hline $\mathrm{C}(11)-\mathrm{C}(35)-\mathrm{C}(36)$ & $112.1(2)$ \\
\hline $\mathrm{C}(37)-\mathrm{C}(35)-\mathrm{C}(36)$ & $108.0(3)$ \\
\hline $\mathrm{C}(35)-\mathrm{C}(36)-\mathrm{H}(36 \mathrm{~A})$ & 109.5 \\
\hline $\mathrm{C}(35)-\mathrm{C}(36)-\mathrm{H}(36 \mathrm{~B})$ & 109.5 \\
\hline
\end{tabular}




\begin{tabular}{|c|c|c|c|}
\hline $\mathrm{H}(36 \mathrm{~A})-\mathrm{C}(36)-\mathrm{H}(36 \mathrm{~B})$ & 109.5 & $\mathrm{H}(42 \mathrm{~A})-\mathrm{C}(42)-\mathrm{H}(42 \mathrm{~B})$ & 109.5 \\
\hline $\mathrm{C}(35)-\mathrm{C}(36)-\mathrm{H}(36 \mathrm{C})$ & 109.5 & $\mathrm{C}(39)-\mathrm{C}(42)-\mathrm{H}(42 \mathrm{C})$ & 109.5 \\
\hline $\mathrm{H}(36 \mathrm{~A})-\mathrm{C}(36)-\mathrm{H}(36 \mathrm{C})$ & 109.5 & $\mathrm{H}(42 \mathrm{~A})-\mathrm{C}(42)-\mathrm{H}(42 \mathrm{C})$ & 109.5 \\
\hline $\mathrm{H}(36 \mathrm{~B})-\mathrm{C}(36)-\mathrm{H}(36 \mathrm{C})$ & 109.5 & $\mathrm{H}(42 \mathrm{~B})-\mathrm{C}(42)-\mathrm{H}(42 \mathrm{C})$ & 109.5 \\
\hline $\mathrm{C}(35)-\mathrm{C}(37)-\mathrm{H}(37 \mathrm{~A})$ & 109.5 & $\mathrm{C}(44)-\mathrm{C}(43)-\mathrm{C}(46)$ & $111.7(3)$ \\
\hline $\mathrm{C}(35)-\mathrm{C}(37)-\mathrm{H}(37 \mathrm{~B})$ & 109.5 & $\mathrm{C}(44)-\mathrm{C}(43)-\mathrm{C}(25)$ & $108.9(2)$ \\
\hline H(37A)-C(37)-H(37B) & 109.5 & $\mathrm{C}(46)-\mathrm{C}(43)-\mathrm{C}(25)$ & $108.8(2)$ \\
\hline $\mathrm{C}(35)-\mathrm{C}(37)-\mathrm{H}(37 \mathrm{C})$ & 109.5 & $\mathrm{C}(44)-\mathrm{C}(43)-\mathrm{C}(45)$ & $108.7(3)$ \\
\hline $\mathrm{H}(37 \mathrm{~A})-\mathrm{C}(37)-\mathrm{H}(37 \mathrm{C})$ & 109.5 & $C(46)-C(43)-C(45)$ & $107.0(3)$ \\
\hline H(37B)-C(37)-H(37C) & 109.5 & $C(25)-C(43)-C(45)$ & $111.7(2)$ \\
\hline $\mathrm{C}(35)-\mathrm{C}(38)-\mathrm{H}(38 \mathrm{~A})$ & 109.5 & $\mathrm{C}(43)-\mathrm{C}(44)-\mathrm{H}(44 \mathrm{~A})$ & 109.5 \\
\hline $\mathrm{C}(35)-\mathrm{C}(38)-\mathrm{H}(38 \mathrm{~B})$ & 109.5 & $\mathrm{C}(43)-\mathrm{C}(44)-\mathrm{H}(44 \mathrm{~B})$ & 109.5 \\
\hline $\mathrm{H}(38 \mathrm{~A})-\mathrm{C}(38)-\mathrm{H}(38 \mathrm{~B})$ & 109.5 & $\mathrm{H}(44 \mathrm{~A})-\mathrm{C}(44)-\mathrm{H}(44 \mathrm{~B})$ & 109.5 \\
\hline $\mathrm{C}(35)-\mathrm{C}(38)-\mathrm{H}(38 \mathrm{C})$ & 109.5 & $\mathrm{C}(43)-\mathrm{C}(44)-\mathrm{H}(44 \mathrm{C})$ & 109.5 \\
\hline $\mathrm{H}(38 \mathrm{~A})-\mathrm{C}(38)-\mathrm{H}(38 \mathrm{C})$ & 109.5 & $\mathrm{H}(44 \mathrm{~A})-\mathrm{C}(44)-\mathrm{H}(44 \mathrm{C})$ & 109.5 \\
\hline $\mathrm{H}(38 \mathrm{~B})-\mathrm{C}(38)-\mathrm{H}(38 \mathrm{C})$ & 109.5 & $\mathrm{H}(44 \mathrm{~B})-\mathrm{C}(44)-\mathrm{H}(44 \mathrm{C})$ & 109.5 \\
\hline $\mathrm{C}(40)-\mathrm{C}(39)-\mathrm{C}(13)$ & $112.2(2)$ & $\mathrm{C}(43)-\mathrm{C}(45)-\mathrm{H}(45 \mathrm{~A})$ & 109.5 \\
\hline $\mathrm{C}(40)-\mathrm{C}(39)-\mathrm{C}(41)$ & $108.1(2)$ & $\mathrm{C}(43)-\mathrm{C}(45)-\mathrm{H}(45 \mathrm{~B})$ & 109.5 \\
\hline $\mathrm{C}(13)-\mathrm{C}(39)-\mathrm{C}(41)$ & $109.1(2)$ & $\mathrm{H}(45 \mathrm{~A})-\mathrm{C}(45)-\mathrm{H}(45 \mathrm{~B})$ & 109.5 \\
\hline $\mathrm{C}(40)-\mathrm{C}(39)-\mathrm{C}(42)$ & $108.7(3)$ & $\mathrm{C}(43)-\mathrm{C}(45)-\mathrm{H}(45 \mathrm{C})$ & 109.5 \\
\hline $\mathrm{C}(13)-\mathrm{C}(39)-\mathrm{C}(42)$ & $109.6(2)$ & $\mathrm{H}(45 \mathrm{~A})-\mathrm{C}(45)-\mathrm{H}(45 \mathrm{C})$ & 109.5 \\
\hline C(41)-C(39)-C(42) & $109.1(2)$ & $\mathrm{H}(45 \mathrm{~B})-\mathrm{C}(45)-\mathrm{H}(45 \mathrm{C})$ & 109.5 \\
\hline $\mathrm{C}(39)-\mathrm{C}(40)-\mathrm{H}(40 \mathrm{~A})$ & 109.5 & $\mathrm{C}(43)-\mathrm{C}(46)-\mathrm{H}(46 \mathrm{~A})$ & 109.5 \\
\hline $\mathrm{C}(39)-\mathrm{C}(40)-\mathrm{H}(40 \mathrm{~B})$ & 109.5 & $\mathrm{C}(43)-\mathrm{C}(46)-\mathrm{H}(46 \mathrm{~B})$ & 109.5 \\
\hline $\mathrm{H}(40 \mathrm{~A})-\mathrm{C}(40)-\mathrm{H}(40 \mathrm{~B})$ & 109.5 & $\mathrm{H}(46 \mathrm{~A})-\mathrm{C}(46)-\mathrm{H}(46 \mathrm{~B})$ & 109.5 \\
\hline $\mathrm{C}(39)-\mathrm{C}(40)-\mathrm{H}(40 \mathrm{C})$ & 109.5 & $\mathrm{C}(43)-\mathrm{C}(46)-\mathrm{H}(46 \mathrm{C})$ & 109.5 \\
\hline $\mathrm{H}(40 \mathrm{~A})-\mathrm{C}(40)-\mathrm{H}(40 \mathrm{C})$ & 109.5 & $\mathrm{H}(46 \mathrm{~A})-\mathrm{C}(46)-\mathrm{H}(46 \mathrm{C})$ & 109.5 \\
\hline $\mathrm{H}(40 \mathrm{~B})-\mathrm{C}(40)-\mathrm{H}(40 \mathrm{C})$ & 109.5 & $\mathrm{H}(46 \mathrm{~B})-\mathrm{C}(46)-\mathrm{H}(46 \mathrm{C})$ & 109.5 \\
\hline $\mathrm{C}(39)-\mathrm{C}(41)-\mathrm{H}(41 \mathrm{~A})$ & 109.5 & $C(50)-C(47)-C(48)$ & $109.0(3)$ \\
\hline $\mathrm{C}(39)-\mathrm{C}(41)-\mathrm{H}(41 \mathrm{~B})$ & 109.5 & $C(50)-C(47)-C(49)$ & $107.7(3)$ \\
\hline $\mathrm{H}(41 \mathrm{~A})-\mathrm{C}(41)-\mathrm{H}(41 \mathrm{~B})$ & 109.5 & $\mathrm{C}(48)-\mathrm{C}(47)-\mathrm{C}(49)$ & $109.1(3)$ \\
\hline $\mathrm{C}(39)-\mathrm{C}(41)-\mathrm{H}(41 \mathrm{C})$ & 109.5 & $\mathrm{C}(50)-\mathrm{C}(47)-\mathrm{C}(23)$ & $111.8(2)$ \\
\hline $\mathrm{H}(41 \mathrm{~A})-\mathrm{C}(41)-\mathrm{H}(41 \mathrm{C})$ & 109.5 & $\mathrm{C}(48)-\mathrm{C}(47)-\mathrm{C}(23)$ & $109.1(2)$ \\
\hline $\mathrm{H}(41 \mathrm{~B})-\mathrm{C}(41)-\mathrm{H}(41 \mathrm{C})$ & 109.5 & $\mathrm{C}(49)-\mathrm{C}(47)-\mathrm{C}(23)$ & $110.0(2)$ \\
\hline $\mathrm{C}(39)-\mathrm{C}(42)-\mathrm{H}(42 \mathrm{~A})$ & 109.5 & $\mathrm{C}(47)-\mathrm{C}(48)-\mathrm{H}(48 \mathrm{~A})$ & 109.5 \\
\hline C(39)-C(42)-H(42B) & 109.5 & $\mathrm{C}(47)-\mathrm{C}(48)-\mathrm{H}(48 \mathrm{~B})$ & 109.5 \\
\hline
\end{tabular}




\begin{tabular}{|c|c|c|c|}
\hline $\mathrm{H}(48 \mathrm{~A})-\mathrm{C}(48)-\mathrm{H}(48 \mathrm{~B})$ & 109.5 & $\mathrm{H}(54 \mathrm{~A})-\mathrm{C}(54)-\mathrm{H}(54 \mathrm{~B})$ & 109.5 \\
\hline $\mathrm{C}(47)-\mathrm{C}(48)-\mathrm{H}(48 \mathrm{C})$ & 109.5 & $\mathrm{C}(51)-\mathrm{C}(54)-\mathrm{H}(54 \mathrm{C})$ & 109.5 \\
\hline $\mathrm{H}(48 \mathrm{~A})-\mathrm{C}(48)-\mathrm{H}(48 \mathrm{C})$ & 109.5 & $\mathrm{H}(54 \mathrm{~A})-\mathrm{C}(54)-\mathrm{H}(54 \mathrm{C})$ & 109.5 \\
\hline $\mathrm{H}(48 \mathrm{~B})-\mathrm{C}(48)-\mathrm{H}(48 \mathrm{C})$ & 109.5 & $\mathrm{H}(54 \mathrm{~B})-\mathrm{C}(54)-\mathrm{H}(54 \mathrm{C})$ & 109.5 \\
\hline $\mathrm{C}(47)-\mathrm{C}(49)-\mathrm{H}(49 \mathrm{~A})$ & 109.5 & $\mathrm{C}(57)-\mathrm{C}(55)-\mathrm{C}(58)$ & $108.2(3)$ \\
\hline $\mathrm{C}(47)-\mathrm{C}(49)-\mathrm{H}(49 \mathrm{~B})$ & 109.5 & $\mathrm{C}(57)-\mathrm{C}(55)-\mathrm{C}(17)$ & $110.6(3)$ \\
\hline $\mathrm{H}(49 \mathrm{~A})-\mathrm{C}(49)-\mathrm{H}(49 \mathrm{~B})$ & 109.5 & $\mathrm{C}(58)-\mathrm{C}(55)-\mathrm{C}(17)$ & $111.9(3)$ \\
\hline $\mathrm{C}(47)-\mathrm{C}(49)-\mathrm{H}(49 \mathrm{C})$ & 109.5 & $\mathrm{C}(57)-\mathrm{C}(55)-\mathrm{C}(56)$ & $110.5(4)$ \\
\hline $\mathrm{H}(49 \mathrm{~A})-\mathrm{C}(49)-\mathrm{H}(49 \mathrm{C})$ & 109.5 & $\mathrm{C}(58)-\mathrm{C}(55)-\mathrm{C}(56)$ & $108.0(3)$ \\
\hline H(49B)-C(49)-H(49C) & 109.5 & $C(17)-C(55)-C(56)$ & $107.6(3)$ \\
\hline $\mathrm{C}(47)-\mathrm{C}(50)-\mathrm{H}(50 \mathrm{~A})$ & 109.5 & $\mathrm{C}(55)-\mathrm{C}(56)-\mathrm{H}(56 \mathrm{~A})$ & 109.5 \\
\hline $\mathrm{C}(47)-\mathrm{C}(50)-\mathrm{H}(50 \mathrm{~B})$ & 109.5 & $\mathrm{C}(55)-\mathrm{C}(56)-\mathrm{H}(56 \mathrm{~B})$ & 109.5 \\
\hline $\mathrm{H}(50 \mathrm{~A})-\mathrm{C}(50)-\mathrm{H}(50 \mathrm{~B})$ & 109.5 & $\mathrm{H}(56 \mathrm{~A})-\mathrm{C}(56)-\mathrm{H}(56 \mathrm{~B})$ & 109.5 \\
\hline $\mathrm{C}(47)-\mathrm{C}(50)-\mathrm{H}(50 \mathrm{C})$ & 109.5 & $\mathrm{C}(55)-\mathrm{C}(56)-\mathrm{H}(56 \mathrm{C})$ & 109.5 \\
\hline $\mathrm{H}(50 \mathrm{~A})-\mathrm{C}(50)-\mathrm{H}(50 \mathrm{C})$ & 109.5 & $\mathrm{H}(56 \mathrm{~A})-\mathrm{C}(56)-\mathrm{H}(56 \mathrm{C})$ & 109.5 \\
\hline $\mathrm{H}(50 \mathrm{~B})-\mathrm{C}(50)-\mathrm{H}(50 \mathrm{C})$ & 109.5 & $\mathrm{H}(56 \mathrm{~B})-\mathrm{C}(56)-\mathrm{H}(56 \mathrm{C})$ & 109.5 \\
\hline $\mathrm{C}(53)-\mathrm{C}(51)-\mathrm{C}(52)$ & $108.6(2)$ & $\mathrm{C}(55)-\mathrm{C}(57)-\mathrm{H}(57 \mathrm{~A})$ & 109.5 \\
\hline $\mathrm{C}(53)-\mathrm{C}(51)-\mathrm{C}(19)$ & $112.1(2)$ & $\mathrm{C}(55)-\mathrm{C}(57)-\mathrm{H}(57 \mathrm{~B})$ & 109.5 \\
\hline $\mathrm{C}(52)-\mathrm{C}(51)-\mathrm{C}(19)$ & $109.2(2)$ & $\mathrm{H}(57 \mathrm{~A})-\mathrm{C}(57)-\mathrm{H}(57 \mathrm{~B})$ & 109.5 \\
\hline$C(53)-C(51)-C(54)$ & $107.9(2)$ & $\mathrm{C}(55)-\mathrm{C}(57)-\mathrm{H}(57 \mathrm{C})$ & 109.5 \\
\hline$C(52)-C(51)-C(54)$ & $109.7(2)$ & $\mathrm{H}(57 \mathrm{~A})-\mathrm{C}(57)-\mathrm{H}(57 \mathrm{C})$ & 109.5 \\
\hline$C(19)-C(51)-C(54)$ & $109.3(2)$ & $\mathrm{H}(57 \mathrm{~B})-\mathrm{C}(57)-\mathrm{H}(57 \mathrm{C})$ & 109.5 \\
\hline $\mathrm{C}(51)-\mathrm{C}(52)-\mathrm{H}(52 \mathrm{~A})$ & 109.5 & $\mathrm{C}(55)-\mathrm{C}(58)-\mathrm{H}(58 \mathrm{~A})$ & 109.5 \\
\hline $\mathrm{C}(51)-\mathrm{C}(52)-\mathrm{H}(52 \mathrm{~B})$ & 109.5 & $\mathrm{C}(55)-\mathrm{C}(58)-\mathrm{H}(58 \mathrm{~B})$ & 109.5 \\
\hline $\mathrm{H}(52 \mathrm{~A})-\mathrm{C}(52)-\mathrm{H}(52 \mathrm{~B})$ & 109.5 & $\mathrm{H}(58 \mathrm{~A})-\mathrm{C}(58)-\mathrm{H}(58 \mathrm{~B})$ & 109.5 \\
\hline $\mathrm{C}(51)-\mathrm{C}(52)-\mathrm{H}(52 \mathrm{C})$ & 109.5 & $\mathrm{C}(55)-\mathrm{C}(58)-\mathrm{H}(58 \mathrm{C})$ & 109.5 \\
\hline $\mathrm{H}(52 \mathrm{~A})-\mathrm{C}(52)-\mathrm{H}(52 \mathrm{C})$ & 109.5 & $\mathrm{H}(58 \mathrm{~A})-\mathrm{C}(58)-\mathrm{H}(58 \mathrm{C})$ & 109.5 \\
\hline $\mathrm{H}(52 \mathrm{~B})-\mathrm{C}(52)-\mathrm{H}(52 \mathrm{C})$ & 109.5 & $\mathrm{H}(58 \mathrm{~B})-\mathrm{C}(58)-\mathrm{H}(58 \mathrm{C})$ & 109.5 \\
\hline $\mathrm{C}(51)-\mathrm{C}(53)-\mathrm{H}(53 \mathrm{~A})$ & 109.5 & $\mathrm{C}\left(57^{\prime}\right)-\mathrm{C}\left(55^{\prime}\right)-\mathrm{C}\left(58^{\prime}\right)$ & $109(2)$ \\
\hline $\mathrm{C}(51)-\mathrm{C}(53)-\mathrm{H}(53 \mathrm{~B})$ & 109.5 & $C\left(57^{\prime}\right)-C\left(55^{\prime}\right)-C(17)$ & $103(3)$ \\
\hline $\mathrm{H}(53 \mathrm{~A})-\mathrm{C}(53)-\mathrm{H}(53 \mathrm{~B})$ & 109.5 & $\mathrm{C}\left(58^{\prime}\right)-\mathrm{C}\left(55^{\prime}\right)-\mathrm{C}(17)$ & $116(3)$ \\
\hline $\mathrm{C}(51)-\mathrm{C}(53)-\mathrm{H}(53 \mathrm{C})$ & 109.5 & $\mathrm{C}\left(57^{\prime}\right)-\mathrm{C}\left(55^{\prime}\right)-\mathrm{C}\left(56^{\prime}\right)$ & $110(2)$ \\
\hline $\mathrm{H}(53 \mathrm{~A})-\mathrm{C}(53)-\mathrm{H}(53 \mathrm{C})$ & 109.5 & $\mathrm{C}\left(58^{\prime}\right)-\mathrm{C}\left(55^{\prime}\right)-\mathrm{C}\left(56^{\prime}\right)$ & $106(2)$ \\
\hline $\mathrm{H}(53 \mathrm{~B})-\mathrm{C}(53)-\mathrm{H}(53 \mathrm{C})$ & 109.5 & $\mathrm{C}(17)-\mathrm{C}\left(55^{\prime}\right)-\mathrm{C}\left(56^{\prime}\right)$ & $113(2)$ \\
\hline $\mathrm{C}(51)-\mathrm{C}(54)-\mathrm{H}(54 \mathrm{~A})$ & 109.5 & $\mathrm{C}\left(55^{\prime}\right)-\mathrm{C}\left(56^{\prime}\right)-\mathrm{H}(56 \mathrm{D})$ & 109.5 \\
\hline $\mathrm{C}(51)-\mathrm{C}(54)-\mathrm{H}(54 \mathrm{~B})$ & 109.5 & $\mathrm{C}\left(55^{\prime}\right)-\mathrm{C}\left(56^{\prime}\right)-\mathrm{H}(56 \mathrm{E})$ & 109.5 \\
\hline
\end{tabular}




\begin{tabular}{|c|c|c|c|}
\hline $\mathrm{H}(56 \mathrm{D})-\mathrm{C}\left(56^{\prime}\right)-\mathrm{H}(56 \mathrm{E})$ & 109.5 & $\mathrm{C}(62)-\mathrm{C}(61)-\mathrm{H}(61 \mathrm{~B})$ & 109.2 \\
\hline $\mathrm{C}\left(55^{\prime}\right)-\mathrm{C}\left(56^{\prime}\right)-\mathrm{H}(56 \mathrm{~F})$ & 109.5 & $\mathrm{H}(61 \mathrm{~A})-\mathrm{C}(61)-\mathrm{H}(61 \mathrm{~B})$ & 107.9 \\
\hline $\mathrm{H}(56 \mathrm{D})-\mathrm{C}\left(56^{\prime}\right)-\mathrm{H}(56 \mathrm{~F})$ & 109.5 & $\mathrm{O}(1)-\mathrm{C}(62)-\mathrm{C}(61)$ & $110.1(3)$ \\
\hline $\mathrm{H}(56 \mathrm{E})-\mathrm{C}\left(56^{\prime}\right)-\mathrm{H}(56 \mathrm{~F})$ & 109.5 & $\mathrm{O}(1)-\mathrm{C}(62)-\mathrm{H}(62 \mathrm{~A})$ & 109.6 \\
\hline $\mathrm{C}\left(55^{\prime}\right)-\mathrm{C}\left(57^{\prime}\right)-\mathrm{H}(57 \mathrm{D})$ & 109.5 & $\mathrm{C}(61)-\mathrm{C}(62)-\mathrm{H}(62 \mathrm{~A})$ & 109.6 \\
\hline $\mathrm{C}\left(55^{\prime}\right)-\mathrm{C}\left(57^{\prime}\right)-\mathrm{H}(57 \mathrm{E})$ & 109.5 & $\mathrm{O}(1)-\mathrm{C}(62)-\mathrm{H}(62 \mathrm{~B})$ & 109.6 \\
\hline $\mathrm{H}(57 \mathrm{D})-\mathrm{C}\left(57^{\prime}\right)-\mathrm{H}(57 \mathrm{E})$ & 109.5 & $\mathrm{C}(61)-\mathrm{C}(62)-\mathrm{H}(62 \mathrm{~B})$ & 109.6 \\
\hline $\mathrm{C}\left(55^{\prime}\right)-\mathrm{C}\left(57^{\prime}\right)-\mathrm{H}(57 \mathrm{~F})$ & 109.5 & $\mathrm{H}(62 \mathrm{~A})-\mathrm{C}(62)-\mathrm{H}(62 \mathrm{~B})$ & 108.2 \\
\hline $\mathrm{H}(57 \mathrm{D})-\mathrm{C}\left(57^{\prime}\right)-\mathrm{H}(57 \mathrm{~F})$ & 109.5 & $\mathrm{C}(63)-\mathrm{O}(3)-\mathrm{C}(66)$ & $108.8(2)$ \\
\hline $\mathrm{H}(57 \mathrm{E})-\mathrm{C}\left(57^{\prime}\right)-\mathrm{H}(57 \mathrm{~F})$ & 109.5 & $\mathrm{O}(3)-\mathrm{C}(63)-\mathrm{C}(64)$ & $110.9(3)$ \\
\hline $\mathrm{C}\left(55^{\prime}\right)-\mathrm{C}\left(58^{\prime}\right)-\mathrm{H}(58 \mathrm{D})$ & 109.5 & $\mathrm{O}(3)-\mathrm{C}(63)-\mathrm{H}(63 \mathrm{~A})$ & 109.5 \\
\hline $\mathrm{C}\left(55^{\prime}\right)-\mathrm{C}\left(58^{\prime}\right)-\mathrm{H}(58 \mathrm{E})$ & 109.5 & $\mathrm{C}(64)-\mathrm{C}(63)-\mathrm{H}(63 \mathrm{~A})$ & 109.5 \\
\hline $\mathrm{H}(58 \mathrm{D})-\mathrm{C}\left(58^{\prime}\right)-\mathrm{H}(58 \mathrm{E})$ & 109.5 & $\mathrm{O}(3)-\mathrm{C}(63)-\mathrm{H}(63 \mathrm{~B})$ & 109.5 \\
\hline $\mathrm{C}\left(55^{\prime}\right)-\mathrm{C}\left(58^{\prime}\right)-\mathrm{H}(58 \mathrm{~F})$ & 109.5 & $\mathrm{C}(64)-\mathrm{C}(63)-\mathrm{H}(63 \mathrm{~B})$ & 109.5 \\
\hline $\mathrm{H}(58 \mathrm{D})-\mathrm{C}\left(58^{\prime}\right)-\mathrm{H}(58 \mathrm{~F})$ & 109.5 & $\mathrm{H}(63 \mathrm{~A})-\mathrm{C}(63)-\mathrm{H}(63 \mathrm{~B})$ & 108.1 \\
\hline $\mathrm{H}(58 \mathrm{E})-\mathrm{C}\left(58^{\prime}\right)-\mathrm{H}(58 \mathrm{~F})$ & 109.5 & $\mathrm{O}(4)-\mathrm{C}(64)-\mathrm{C}(63)$ & $111.2(3)$ \\
\hline $\mathrm{C}(62)-\mathrm{O}(1)-\mathrm{C}(59)$ & $108.9(3)$ & $\mathrm{O}(4)-\mathrm{C}(64)-\mathrm{H}(64 \mathrm{~A})$ & 109.4 \\
\hline $\mathrm{O}(1)-\mathrm{C}(59)-\mathrm{C}(60)$ & $110.3(3)$ & $\mathrm{C}(63)-\mathrm{C}(64)-\mathrm{H}(64 \mathrm{~A})$ & 109.4 \\
\hline $\mathrm{O}(1)-\mathrm{C}(59)-\mathrm{H}(59 \mathrm{~A})$ & 109.6 & $\mathrm{O}(4)-\mathrm{C}(64)-\mathrm{H}(64 \mathrm{~B})$ & 109.4 \\
\hline $\mathrm{C}(60)-\mathrm{C}(59)-\mathrm{H}(59 \mathrm{~A})$ & 109.6 & $\mathrm{C}(63)-\mathrm{C}(64)-\mathrm{H}(64 \mathrm{~B})$ & 109.4 \\
\hline $\mathrm{O}(1)-\mathrm{C}(59)-\mathrm{H}(59 \mathrm{~B})$ & 109.6 & $\mathrm{H}(64 \mathrm{~A})-\mathrm{C}(64)-\mathrm{H}(64 \mathrm{~B})$ & 108.0 \\
\hline $\mathrm{C}(60)-\mathrm{C}(59)-\mathrm{H}(59 \mathrm{~B})$ & 109.6 & $\mathrm{C}(65)-\mathrm{O}(4)-\mathrm{C}(64)$ & $109.3(2)$ \\
\hline H(59A)-C(59)-H(59B) & 108.1 & $\mathrm{O}(4)-\mathrm{C}(65)-\mathrm{C}(66)$ & $110.9(3)$ \\
\hline $\mathrm{O}(2)-\mathrm{C}(60)-\mathrm{C}(59)$ & $111.3(3)$ & $\mathrm{O}(4)-\mathrm{C}(65)-\mathrm{H}(65 \mathrm{~A})$ & 109.5 \\
\hline $\mathrm{O}(2)-\mathrm{C}(60)-\mathrm{H}(60 \mathrm{~A})$ & 109.4 & $\mathrm{C}(66)-\mathrm{C}(65)-\mathrm{H}(65 \mathrm{~A})$ & 109.5 \\
\hline$C(59)-C(60)-H(60 A)$ & 109.4 & $\mathrm{O}(4)-\mathrm{C}(65)-\mathrm{H}(65 \mathrm{~B})$ & 109.5 \\
\hline $\mathrm{O}(2)-\mathrm{C}(60)-\mathrm{H}(60 \mathrm{~B})$ & 109.4 & $\mathrm{C}(66)-\mathrm{C}(65)-\mathrm{H}(65 \mathrm{~B})$ & 109.5 \\
\hline $\mathrm{C}(59)-\mathrm{C}(60)-\mathrm{H}(60 \mathrm{~B})$ & 109.4 & $\mathrm{H}(65 \mathrm{~A})-\mathrm{C}(65)-\mathrm{H}(65 \mathrm{~B})$ & 108.0 \\
\hline $\mathrm{H}(60 \mathrm{~A})-\mathrm{C}(60)-\mathrm{H}(60 \mathrm{~B})$ & 108.0 & $\mathrm{O}(3)-\mathrm{C}(66)-\mathrm{C}(65)$ & $110.5(3)$ \\
\hline $\mathrm{C}(61)-\mathrm{O}(2)-\mathrm{C}(60)$ & $108.9(3)$ & $\mathrm{O}(3)-\mathrm{C}(66)-\mathrm{H}(66 \mathrm{~A})$ & 109.5 \\
\hline $\mathrm{O}(2)-\mathrm{C}(61)-\mathrm{C}(62)$ & $111.9(3)$ & $\mathrm{C}(65)-\mathrm{C}(66)-\mathrm{H}(66 \mathrm{~A})$ & 109.5 \\
\hline $\mathrm{O}(2)-\mathrm{C}(61)-\mathrm{H}(61 \mathrm{~A})$ & 109.2 & $\mathrm{O}(3)-\mathrm{C}(66)-\mathrm{H}(66 \mathrm{~B})$ & 109.5 \\
\hline$C(62)-C(61)-H(61 A)$ & 109.2 & $\mathrm{C}(65)-\mathrm{C}(66)-\mathrm{H}(66 \mathrm{~B})$ & 109.5 \\
\hline $\mathrm{O}(2)-\mathrm{C}(61)-\mathrm{H}(61 \mathrm{~B})$ & 109.2 & $\mathrm{H}(66 \mathrm{~A})-\mathrm{C}(66)-\mathrm{H}(66 \mathrm{~B})$ & 108.1 \\
\hline
\end{tabular}


Table S10. Anisotropic displacement parameters $\left(\AA^{2} \times 10^{3}\right)$ for neijk04. The anisotropic displacement factor exponent takes the form: $-2 \pi^{2}\left[h^{2} a^{* 2} U_{11}+\ldots+2 h k a^{*} b^{*} U_{12}\right]$

\begin{tabular}{|c|c|c|c|c|c|c|}
\hline & $\mathrm{U}_{11}$ & $\mathrm{U}_{22}$ & $\mathrm{U}_{33}$ & $\mathrm{U}_{23}$ & $\mathrm{U}_{13}$ & $\mathrm{U}_{12}$ \\
\hline $\mathrm{Fe} 1$ & $23(1)$ & 21(1) & $19(1)$ & $6(1)$ & $10(1)$ & $5(1)$ \\
\hline $\mathrm{Cl1}$ & $24(1)$ & $39(1)$ & $40(1)$ & 15(1) & $14(1)$ & $6(1)$ \\
\hline $\mathrm{Cl} 2$ & $48(1)$ & $35(1)$ & $17(1)$ & $0(1)$ & $7(1)$ & $10(1)$ \\
\hline $\mathrm{P} 1$ & $18(1)$ & $14(1)$ & $17(1)$ & $1(1)$ & $1(1)$ & $0(1)$ \\
\hline $\mathrm{P} 2$ & $21(1)$ & $14(1)$ & $14(1)$ & $2(1)$ & $7(1)$ & 1(1) \\
\hline $\mathrm{C} 1$ & $25(1)$ & 19(1) & $16(1)$ & $-1(1)$ & $9(1)$ & $-1(1)$ \\
\hline $\mathrm{C} 2$ & $22(1)$ & 19(1) & $15(1)$ & $1(1)$ & $3(1)$ & $0(1)$ \\
\hline $\mathrm{C} 3$ & $21(1)$ & 19(1) & $17(1)$ & $-1(1)$ & $6(1)$ & $-3(1)$ \\
\hline $\mathrm{C} 4$ & $20(1)$ & 21(1) & $17(1)$ & $1(1)$ & $4(1)$ & 1(1) \\
\hline $\mathrm{C} 5$ & $21(1)$ & $18(1)$ & $18(1)$ & $1(1)$ & $5(1)$ & $2(1)$ \\
\hline C6 & $23(1)$ & 21(1) & $18(1)$ & $-2(1)$ & $7(1)$ & $-4(1)$ \\
\hline C7 & $19(1)$ & 23(1) & $18(1)$ & $2(1)$ & $4(1)$ & $0(1)$ \\
\hline $\mathrm{C} 8$ & $16(1)$ & 19(1) & $17(1)$ & $3(1)$ & $0(1)$ & $2(1)$ \\
\hline C9 & $17(1)$ & $15(1)$ & $18(1)$ & $0(1)$ & $0(1)$ & $-2(1)$ \\
\hline $\mathrm{C} 10$ & $22(1)$ & 19(1) & $18(1)$ & $1(1)$ & $6(1)$ & 1(1) \\
\hline $\mathrm{C} 11$ & $25(1)$ & 19(1) & $14(1)$ & $-1(1)$ & $1(1)$ & $0(1)$ \\
\hline $\mathrm{C} 12$ & $17(1)$ & $23(1)$ & $21(2)$ & $-2(1)$ & $0(1)$ & $2(1)$ \\
\hline $\mathrm{C} 13$ & $20(1)$ & 19(1) & $19(1)$ & $0(1)$ & $4(1)$ & $-2(1)$ \\
\hline $\mathrm{C} 14$ & $20(1)$ & $17(1)$ & $14(1)$ & $2(1)$ & $3(1)$ & $-4(1)$ \\
\hline $\mathrm{C} 15$ & $18(1)$ & $16(1)$ & $16(1)$ & $1(1)$ & $4(1)$ & $-3(1)$ \\
\hline C16 & $26(1)$ & 21(1) & $15(1)$ & $5(1)$ & $6(1)$ & 1(1) \\
\hline $\mathrm{C} 17$ & $23(1)$ & $28(2)$ & $21(2)$ & $3(1)$ & $9(1)$ & $3(1)$ \\
\hline C18 & $21(1)$ & 21(1) & $19(1)$ & $3(1)$ & $3(1)$ & $0(1)$ \\
\hline C19 & $23(1)$ & $17(1)$ & $16(1)$ & $0(1)$ & $4(1)$ & $-4(1)$ \\
\hline $\mathrm{C} 20$ & $22(1)$ & $15(1)$ & $17(1)$ & $-2(1)$ & $5(1)$ & $-3(1)$ \\
\hline $\mathrm{C} 21$ & $22(1)$ & $18(1)$ & $12(1)$ & $-1(1)$ & $7(1)$ & $2(1)$ \\
\hline $\mathrm{C} 22$ & $21(1)$ & $18(1)$ & $14(1)$ & $2(1)$ & $7(1)$ & $5(1)$ \\
\hline $\mathrm{C} 23$ & $27(1)$ & 19(1) & $14(1)$ & $-1(1)$ & $10(1)$ & $0(1)$ \\
\hline $\mathrm{C} 24$ & $22(1)$ & $25(1)$ & $17(1)$ & $-4(1)$ & $7(1)$ & $-2(1)$ \\
\hline $\mathrm{C} 25$ & $20(1)$ & $28(2)$ & 11(1) & $-5(1)$ & $5(1)$ & $4(1)$ \\
\hline C26 & $27(1)$ & $20(1)$ & 11(1) & $1(1)$ & $6(1)$ & $6(1)$ \\
\hline
\end{tabular}




\begin{tabular}{|c|c|c|c|c|c|c|}
\hline $\mathrm{C} 27$ & $18(1)$ & $21(1)$ & $25(2)$ & $5(1)$ & $-1(1)$ & $-3(1)$ \\
\hline $\mathrm{C} 28$ & $34(2)$ & $37(2)$ & $111(4)$ & $23(2)$ & $-26(2)$ & $-14(2)$ \\
\hline $\mathrm{C} 29$ & $29(2)$ & $73(3)$ & $24(2)$ & $-6(2)$ & $-1(1)$ & $3(2)$ \\
\hline $\mathrm{C} 30$ & $19(1)$ & $59(2)$ & $31(2)$ & $-2(2)$ & $1(1)$ & $5(1)$ \\
\hline C31 & $22(1)$ & $19(1)$ & $26(2)$ & $0(1)$ & $3(1)$ & $2(1)$ \\
\hline C32 & $34(2)$ & $20(2)$ & $42(2)$ & $6(1)$ & $-4(2)$ & $0(1)$ \\
\hline C33 & $45(2)$ & $24(2)$ & $22(2)$ & $3(1)$ & $-4(1)$ & $3(1)$ \\
\hline $\mathrm{C} 34$ & $38(2)$ & $39(2)$ & $51(2)$ & $13(2)$ & $20(2)$ & $18(2)$ \\
\hline C35 & $31(2)$ & $30(2)$ & $15(1)$ & $1(1)$ & $-1(1)$ & $6(1)$ \\
\hline C36 & $53(2)$ & $49(2)$ & $20(2)$ & $-5(2)$ & $-6(2)$ & $25(2)$ \\
\hline C37 & $60(2)$ & $52(2)$ & $20(2)$ & $11(2)$ & $4(2)$ & $-9(2)$ \\
\hline C38 & $50(2)$ & $31(2)$ & $20(2)$ & $-2(1)$ & $-3(1)$ & $10(2)$ \\
\hline C39 & $22(1)$ & $26(2)$ & $24(2)$ & $-1(1)$ & $9(1)$ & $0(1)$ \\
\hline $\mathrm{C} 40$ & $35(2)$ & $48(2)$ & $26(2)$ & $-2(2)$ & $14(1)$ & $8(2)$ \\
\hline C41 & $34(2)$ & $30(2)$ & $34(2)$ & $-2(1)$ & $16(1)$ & $3(1)$ \\
\hline C42 & $30(2)$ & $33(2)$ & $46(2)$ & $-5(2)$ & $19(2)$ & $-2(1)$ \\
\hline C43 & $20(1)$ & $34(2)$ & $18(1)$ & $-1(1)$ & $2(1)$ & $3(1)$ \\
\hline C44 & $31(2)$ & $40(2)$ & $50(2)$ & $-17(2)$ & $-7(2)$ & $15(1)$ \\
\hline $\mathrm{C} 45$ & $24(2)$ & $46(2)$ & $43(2)$ & $-14(2)$ & $-1(1)$ & $0(1)$ \\
\hline C46 & $36(2)$ & $69(3)$ & $29(2)$ & $12(2)$ & $-4(2)$ & $2(2)$ \\
\hline C47 & $28(1)$ & $16(1)$ & $30(2)$ & $2(1)$ & $9(1)$ & $-2(1)$ \\
\hline C48 & $79(3)$ & $19(2)$ & $48(2)$ & $-4(2)$ & $36(2)$ & $-5(2)$ \\
\hline C49 & $39(2)$ & $26(2)$ & $30(2)$ & $4(1)$ & $14(1)$ & $-4(1)$ \\
\hline C50 & $37(2)$ & $29(2)$ & $71(3)$ & $26(2)$ & $10(2)$ & $1(1)$ \\
\hline C51 & $31(2)$ & $22(1)$ & $15(1)$ & $3(1)$ & $3(1)$ & $2(1)$ \\
\hline C52 & $38(2)$ & $31(2)$ & $17(2)$ & $1(1)$ & $-6(1)$ & $1(1)$ \\
\hline C53 & $42(2)$ & $30(2)$ & $16(2)$ & $3(1)$ & $9(1)$ & $5(1)$ \\
\hline C54 & $39(2)$ & $25(2)$ & $24(2)$ & $4(1)$ & $12(1)$ & $5(1)$ \\
\hline C55 & $29(2)$ & $45(2)$ & $22(2)$ & $11(2)$ & $12(2)$ & $17(2)$ \\
\hline C56 & $50(3)$ & $64(3)$ & $65(5)$ & $44(3)$ & $43(3)$ & $32(3)$ \\
\hline C57 & $49(4)$ & $93(4)$ & $22(2)$ & $-16(2)$ & $3(2)$ & $37(3)$ \\
\hline C58 & $32(2)$ & $40(2)$ & $25(3)$ & $6(2)$ & $11(2)$ & $13(2)$ \\
\hline C55' & $29(2)$ & $45(2)$ & $22(2)$ & $11(2)$ & $12(2)$ & $17(2)$ \\
\hline C56' & $50(3)$ & $64(3)$ & $65(5)$ & $44(3)$ & $43(3)$ & $32(3)$ \\
\hline C57' & $49(4)$ & $93(4)$ & $22(2)$ & $-16(2)$ & $3(2)$ & $37(3)$ \\
\hline C58' & $32(2)$ & $40(2)$ & $25(3)$ & $6(2)$ & $11(2)$ & $13(2)$ \\
\hline
\end{tabular}




\begin{tabular}{lcccccc} 
O1 & $56(2)$ & $57(2)$ & $44(2)$ & $-4(1)$ & $18(1)$ & $-12(1)$ \\
$\mathrm{C} 59$ & $65(3)$ & $50(2)$ & $36(2)$ & $15(2)$ & $22(2)$ & $8(2)$ \\
$\mathrm{C} 60$ & $50(2)$ & $76(3)$ & $42(2)$ & $-13(2)$ & $22(2)$ & $-7(2)$ \\
$\mathrm{O} 2$ & $42(2)$ & $116(3)$ & $44(2)$ & $3(2)$ & $12(1)$ & $6(2)$ \\
$\mathrm{C} 61$ & $39(2)$ & $86(3)$ & $32(2)$ & $14(2)$ & $11(2)$ & $11(2)$ \\
$\mathrm{C} 62$ & $74(3)$ & $65(3)$ & $34(2)$ & $-9(2)$ & $24(2)$ & $9(2)$ \\
O3 & $28(1)$ & $36(1)$ & $33(1)$ & $7(1)$ & $-2(1)$ & $5(1)$ \\
$\mathrm{C} 63$ & $23(2)$ & $47(2)$ & $30(2)$ & $10(2)$ & $0(1)$ & $-3(1)$ \\
$\mathrm{C} 64$ & $34(2)$ & $53(2)$ & $31(2)$ & $3(2)$ & $2(2)$ & $-6(2)$ \\
O4 & $28(1)$ & $50(2)$ & $43(2)$ & $9(1)$ & $-5(1)$ & $6(1)$ \\
C65 & $34(2)$ & $56(2)$ & $43(2)$ & $3(2)$ & $4(2)$ & $10(2)$ \\
C66 & $36(2)$ & $44(2)$ & $42(2)$ & $-6(2)$ & $-1(2)$ & $8(2)$ \\
& & & & & & \\
\hline
\end{tabular}


Table S11. Hydrogen coordinates $\left(\times 10^{4}\right)$ and isotropic displacement parameters $\left(\AA^{2} \times 10^{3}\right)$ for neijk04.

\begin{tabular}{|c|c|c|c|c|}
\hline & $\mathrm{x}$ & $\mathrm{y}$ & z & $\mathrm{U}(\mathrm{eq})$ \\
\hline H1A & 6239 & 2314 & 7140 & 23 \\
\hline H1B & 7143 & 2176 & 7850 & 23 \\
\hline $\mathrm{H} 2 \mathrm{~A}$ & 8044 & 2803 & 7559 & 23 \\
\hline H2B & 7003 & 3005 & 7613 & 23 \\
\hline H4 & 6583 & 3955 & 5719 & 23 \\
\hline H6 & 8896 & 4773 & 6609 & 24 \\
\hline H8 & 8995 & 3397 & 7144 & 21 \\
\hline $\mathrm{H} 10$ & 6096 & 3091 & 4708 & 23 \\
\hline H12 & 3391 & 3439 & 4873 & 25 \\
\hline H14 & 5427 & 3209 & 6915 & 21 \\
\hline H16 & 8994 & 1514 & 6130 & 24 \\
\hline H18 & 10525 & 855 & 8099 & 25 \\
\hline $\mathrm{H} 20$ & 8177 & 1610 & 8278 & 22 \\
\hline $\mathrm{H} 22$ & 7209 & 942 & 7006 & 21 \\
\hline $\mathrm{H} 24$ & 4417 & 646 & 5983 & 25 \\
\hline $\mathrm{H} 26$ & 5489 & 1920 & 5691 & 23 \\
\hline $\mathrm{H} 28 \mathrm{~A}$ & 10121 & 4860 & 7652 & 101 \\
\hline H28B & 10585 & 4693 & 6914 & 101 \\
\hline $\mathrm{H} 28 \mathrm{C}$ & 11186 & 4639 & 7829 & 101 \\
\hline $\mathrm{H} 29 \mathrm{~A}$ & 9608 & 4264 & 8561 & 65 \\
\hline H29B & 10701 & 4078 & 8742 & 65 \\
\hline $\mathrm{H} 29 \mathrm{C}$ & 9822 & 3726 & 8429 & 65 \\
\hline H30A & 11446 & 3834 & 7596 & 55 \\
\hline Н30B & 10812 & 3846 & 6688 & 55 \\
\hline H30C & 10564 & 3478 & 7327 & 55 \\
\hline $\mathrm{H} 32 \mathrm{~A}$ & 8194 & 5236 & 5439 & 51 \\
\hline H32B & 7921 & 5366 & 6285 & 51 \\
\hline $\mathrm{H} 32 \mathrm{C}$ & 7249 & 5541 & 5449 & 51 \\
\hline H33A & 7213 & 4665 & 4513 & 48 \\
\hline H33B & 6310 & 5003 & 4503 & 48 \\
\hline
\end{tabular}




\begin{tabular}{|c|c|c|c|c|}
\hline $\mathrm{H} 33 \mathrm{C}$ & 6239 & 4464 & 4721 & 48 \\
\hline $\mathrm{H} 34 \mathrm{~A}$ & 6455 & 5020 & 6645 & 62 \\
\hline H34B & 5807 & 4658 & 6037 & 62 \\
\hline $\mathrm{H} 34 \mathrm{C}$ & 5822 & 5197 & 5795 & 62 \\
\hline H36A & 2937 & 3381 & 3600 & 64 \\
\hline H36B & 3287 & 3551 & 2806 & 64 \\
\hline $\mathrm{H} 36 \mathrm{C}$ & 3477 & 3871 & 3601 & 64 \\
\hline $\mathrm{H} 37 \mathrm{~A}$ & 5818 & 3424 & 3546 & 68 \\
\hline H37B & 5252 & 3898 & 3629 & 68 \\
\hline $\mathrm{H} 37 \mathrm{C}$ & 5005 & 3608 & 2799 & 68 \\
\hline H38A & 3840 & 2647 & 3526 & 53 \\
\hline H38B & 4981 & 2657 & 3565 & 53 \\
\hline H38C & 4240 & 2834 & 2767 & 53 \\
\hline $\mathrm{H} 40 \mathrm{~A}$ & 4409 & 3668 & 7520 & 53 \\
\hline $\mathrm{H} 40 \mathrm{~B}$ & 4216 & 3119 & 7522 & 53 \\
\hline $\mathrm{H} 40 \mathrm{C}$ & 3394 & 3475 & 7655 & 53 \\
\hline H41A & 3618 & 4155 & 6351 & 48 \\
\hline H41B & 2613 & 3995 & 6557 & 48 \\
\hline $\mathrm{H} 41 \mathrm{C}$ & 2787 & 3934 & 5656 & 48 \\
\hline $\mathrm{H} 42 \mathrm{~A}$ & 2958 & 2763 & 6379 & 53 \\
\hline H42B & 2405 & 3091 & 5660 & 53 \\
\hline $\mathrm{H} 42 \mathrm{C}$ & 2197 & 3141 & 6552 & 53 \\
\hline $\mathrm{H} 44 \mathrm{~A}$ & 3938 & 2111 & 5820 & 64 \\
\hline H44B & 3306 & 1747 & 6205 & 64 \\
\hline $\mathrm{H} 44 \mathrm{C}$ & 2854 & 1993 & 5356 & 64 \\
\hline $\mathrm{H} 45 \mathrm{~A}$ & 3293 & 823 & 4815 & 58 \\
\hline H45B & 2450 & 1199 & 4781 & 58 \\
\hline $\mathrm{H} 45 \mathrm{C}$ & 2957 & 966 & 5628 & 58 \\
\hline $\mathrm{H} 46 \mathrm{~A}$ & 4296 & 1378 & 4191 & 70 \\
\hline H46B & 4360 & 1909 & 4486 & 70 \\
\hline $\mathrm{H} 46 \mathrm{C}$ & 3334 & 1688 & 4069 & 70 \\
\hline $\mathrm{H} 48 \mathrm{~A}$ & 5143 & -82 & 5833 & 68 \\
\hline H48B & 5854 & -447 & 6382 & 68 \\
\hline $\mathrm{H} 48 \mathrm{C}$ & 6278 & -74 & 5845 & 68 \\
\hline H49A & 4558 & 173 & 7074 & 46 \\
\hline H49B & 5330 & 333 & 7868 & 46 \\
\hline
\end{tabular}




\begin{tabular}{|c|c|c|c|c|}
\hline $\mathrm{H} 49 \mathrm{C}$ & 5274 & -201 & 7592 & 46 \\
\hline $\mathrm{H} 50 \mathrm{~A}$ & 7456 & 197 & 7098 & 69 \\
\hline H50B & 7018 & -188 & 7602 & 69 \\
\hline $\mathrm{H} 50 \mathrm{C}$ & 7062 & 345 & 7883 & 69 \\
\hline $\mathrm{H} 52 \mathrm{~A}$ & 10678 & 1163 & 10241 & 46 \\
\hline H52B & 10558 & 1600 & 9639 & 46 \\
\hline $\mathrm{H} 52 \mathrm{C}$ & 11050 & 1131 & 9411 & 46 \\
\hline $\mathrm{H} 53 \mathrm{~A}$ & 8150 & 1154 & 9389 & 43 \\
\hline H53B & 8778 & 1620 & 9594 & 43 \\
\hline $\mathrm{H} 53 \mathrm{C}$ & 8941 & 1195 & 10222 & 43 \\
\hline H54A & 8889 & 430 & 9022 & 43 \\
\hline H54B & 9642 & 457 & 9879 & 43 \\
\hline $\mathrm{H} 54 \mathrm{C}$ & 10028 & 408 & 9058 & 43 \\
\hline H56A & 10470 & 1610 & 5833 & 83 \\
\hline H56B & 11453 & 1330 & 5848 & 83 \\
\hline $\mathrm{H} 56 \mathrm{C}$ & 11320 & 1615 & 6632 & 83 \\
\hline H57A & 9473 & 890 & 5457 & 84 \\
\hline H57B & 9701 & 429 & 5997 & 84 \\
\hline $\mathrm{H} 57 \mathrm{C}$ & 10430 & 594 & 5447 & 84 \\
\hline $\mathrm{H} 58 \mathrm{~A}$ & 11745 & 870 & 7422 & 47 \\
\hline H58B & 11818 & 610 & 6599 & 47 \\
\hline $\mathrm{H} 58 \mathrm{C}$ & 11125 & 409 & 7152 & 47 \\
\hline $\mathrm{H} 56 \mathrm{D}$ & 10876 & 1768 & 6406 & 83 \\
\hline $\mathrm{H} 56 \mathrm{E}$ & 11709 & 1463 & 6151 & 83 \\
\hline $\mathrm{H} 56 \mathrm{~F}$ & 11785 & 1598 & 7083 & 83 \\
\hline H57D & 9992 & 1073 & 5521 & 84 \\
\hline H57E & 9946 & 582 & 5958 & 84 \\
\hline $\mathrm{H} 57 \mathrm{~F}$ & 10897 & 725 & 5645 & 84 \\
\hline $\mathrm{H} 58 \mathrm{D}$ & 11707 & 841 & 7759 & 47 \\
\hline $\mathrm{H} 58 \mathrm{E}$ & 12127 & 736 & 6969 & 47 \\
\hline $\mathrm{H} 58 \mathrm{~F}$ & 11280 & 418 & 7171 & 47 \\
\hline H59A & 6859 & 3806 & 9614 & 59 \\
\hline H59B & 6068 & 4147 & 9854 & 59 \\
\hline $\mathrm{H} 60 \mathrm{~A}$ & 6925 & 4793 & 9562 & 65 \\
\hline H60B & 7606 & 4475 & 10233 & 65 \\
\hline H61A & 7896 & 4419 & 7981 & 62 \\
\hline
\end{tabular}




$\begin{array}{lrlll}\text { H61B } & 7101 & 4760 & 8214 & 62 \\ \text { H62A } & 6354 & 4096 & 7582 & 67 \\ \text { H62B } & 7024 & 3773 & 8250 & 67 \\ \text { H63A } & 9735 & 2315 & 8923 & 41 \\ \text { H63B } & 9468 & 2848 & 9052 & 41 \\ \text { H64A } & 11109 & 3055 & 9319 & 48 \\ \text { H64B } & 10956 & 2647 & 9927 & 48 \\ \text { H65A } & 12043 & 2334 & 8035 & 54 \\ \text { H65B } & 11747 & 2862 & 8168 & 54 \\ \text { H66A } & 10552 & 2502 & 7167 & 51 \\ \text { H66B } & 10404 & 2110 & 7805 & 51 \\ \end{array}$


Table S12. Torsion angles $\left[^{\circ}\right]$ for neijk04.

\begin{tabular}{|c|c|c|c|}
\hline C15-P2-C1-C2 & $95.7(2)$ & C9-C10-C11-C35 & $179.4(2)$ \\
\hline $\mathrm{C} 21-\mathrm{P} 2-\mathrm{C} 1-\mathrm{C} 2$ & $-155.27(19)$ & $\mathrm{C} 10-\mathrm{C} 11-\mathrm{C} 12-\mathrm{C} 13$ & $1.5(4)$ \\
\hline $\mathrm{Fe} 1-\mathrm{P} 2-\mathrm{C} 1-\mathrm{C} 2$ & $-27.0(2)$ & C35-C11-C12-C13 & $179.8(3)$ \\
\hline P2-C1-C2-P1 & $50.1(2)$ & $\mathrm{C} 11-\mathrm{C} 12-\mathrm{C} 13-\mathrm{C} 14$ & $0.1(4)$ \\
\hline C9-P1-C2-C1 & $70.6(2)$ & C11-C12-C13-C39 & $179.2(3)$ \\
\hline $\mathrm{C} 3-\mathrm{P} 1-\mathrm{C} 2-\mathrm{C} 1$ & $-178.80(18)$ & $\mathrm{C} 12-\mathrm{C} 13-\mathrm{C} 14-\mathrm{C} 9$ & $-1.0(4)$ \\
\hline Fe1-P1-C2-C1 & $-48.05(19)$ & C39-C13-C14-C9 & $180.0(2)$ \\
\hline C9-P1-C3-C8 & $165.7(2)$ & C10-C9-C14-C13 & $0.3(4)$ \\
\hline C2-P1-C3-C8 & $51.9(2)$ & P1-C9-C14-C13 & $175.2(2)$ \\
\hline Fe1-P1-C3-C8 & $-66.8(2)$ & $\mathrm{C} 21-\mathrm{P} 2-\mathrm{C} 15-\mathrm{C} 16$ & $95.6(2)$ \\
\hline C9-P1-C3-C4 & $-17.2(3)$ & $\mathrm{C} 1-\mathrm{P} 2-\mathrm{C} 15-\mathrm{C} 16$ & $-157.3(2)$ \\
\hline C2-P1-C3-C4 & $-131.0(2)$ & Fe1-P2-C15-C16 & $-38.3(2)$ \\
\hline Fe1-P1-C3-C4 & $110.3(2)$ & $\mathrm{C} 21-\mathrm{P} 2-\mathrm{C} 15-\mathrm{C} 20$ & $-79.0(2)$ \\
\hline $\mathrm{C} 8-\mathrm{C} 3-\mathrm{C} 4-\mathrm{C} 5$ & $0.9(4)$ & $\mathrm{C} 1-\mathrm{P} 2-\mathrm{C} 15-\mathrm{C} 20$ & 28.1(3) \\
\hline $\mathrm{P} 1-\mathrm{C} 3-\mathrm{C} 4-\mathrm{C} 5$ & $-176.2(2)$ & Fe1-P2-C15-C20 & 147.11(19) \\
\hline C3-C4-C5-C6 & $-0.1(4)$ & $\mathrm{C} 20-\mathrm{C} 15-\mathrm{C} 16-\mathrm{C} 17$ & $0.3(4)$ \\
\hline $\mathrm{C} 3-\mathrm{C} 4-\mathrm{C} 5-\mathrm{C} 31$ & $-179.6(2)$ & $\mathrm{P} 2-\mathrm{C} 15-\mathrm{C} 16-\mathrm{C} 17$ & $-174.5(2)$ \\
\hline $\mathrm{C} 4-\mathrm{C} 5-\mathrm{C} 6-\mathrm{C} 7$ & $-1.2(4)$ & C15-C16-C17-C18 & $0.3(4)$ \\
\hline C31-C5-C6-C7 & $178.3(3)$ & $\mathrm{C} 15-\mathrm{C} 16-\mathrm{C} 17-\mathrm{C} 55$ & $-179.3(4)$ \\
\hline $\mathrm{C} 5-\mathrm{C} 6-\mathrm{C} 7-\mathrm{C} 8$ & $1.7(4)$ & C15-C16-C17-C55' & $-161(2)$ \\
\hline $\mathrm{C} 5-\mathrm{C} 6-\mathrm{C} 7-\mathrm{C} 27$ & $-176.4(3)$ & C16-C17-C18-C19 & $-0.6(4)$ \\
\hline $\mathrm{C} 4-\mathrm{C} 3-\mathrm{C} 8-\mathrm{C} 7$ & $-0.4(4)$ & C55-C17-C18-C19 & $178.9(4)$ \\
\hline $\mathrm{P} 1-\mathrm{C} 3-\mathrm{C} 8-\mathrm{C} 7$ & $176.7(2)$ & C55'-C17-C18-C19 & $163.0(17)$ \\
\hline $\mathrm{C} 6-\mathrm{C} 7-\mathrm{C} 8-\mathrm{C} 3$ & $-0.8(4)$ & C17-C18-C19-C20 & $0.4(4)$ \\
\hline $\mathrm{C} 27-\mathrm{C} 7-\mathrm{C} 8-\mathrm{C} 3$ & $177.3(2)$ & C17-C18-C19-C51 & $179.7(2)$ \\
\hline C3-P1-C9-C10 & $90.2(2)$ & C18-C19-C20-C15 & $0.2(4)$ \\
\hline C2-P1-C9-C10 & $-159.47(19)$ & C51-C19-C20-C15 & $-179.2(2)$ \\
\hline Fe1-P1-C9-C10 & $-44.6(2)$ & C16-C15-C20-C19 & $-0.5(4)$ \\
\hline C3-P1-C9-C14 & $-84.9(2)$ & P2-C15-C20-C19 & $173.9(2)$ \\
\hline C2-P1-C9-C14 & $25.4(3)$ & $\mathrm{C} 15-\mathrm{P} 2-\mathrm{C} 21-\mathrm{C} 26$ & $-176.4(2)$ \\
\hline Fe1-P1-C9-C14 & $140.2(2)$ & $\mathrm{C} 1-\mathrm{P} 2-\mathrm{C} 21-\mathrm{C} 26$ & $70.5(2)$ \\
\hline C14-C9-C10-C11 & $1.4(4)$ & Fe1-P2-C21-C26 & $-47.6(2)$ \\
\hline P1-C9-C10-C11 & $-174.0(2)$ & $\mathrm{C} 15-\mathrm{P} 2-\mathrm{C} 21-\mathrm{C} 22$ & $5.9(2)$ \\
\hline C9-C10-C11-C12 & $-2.2(4)$ & $\mathrm{C} 1-\mathrm{P} 2-\mathrm{C} 21-\mathrm{C} 22$ & $-107.2(2)$ \\
\hline
\end{tabular}




\begin{tabular}{|c|c|c|c|}
\hline $\mathrm{Fe} 1-\mathrm{P} 2-\mathrm{C} 21-\mathrm{C} 22$ & $134.70(19)$ & C12-C13-C39-C42 & $62.8(3)$ \\
\hline $\mathrm{C} 26-\mathrm{C} 21-\mathrm{C} 22-\mathrm{C} 23$ & $-0.5(4)$ & $\mathrm{C} 24-\mathrm{C} 25-\mathrm{C} 43-\mathrm{C} 44$ & $116.8(3)$ \\
\hline $\mathrm{P} 2-\mathrm{C} 21-\mathrm{C} 22-\mathrm{C} 23$ & $177.1(2)$ & $\mathrm{C} 26-\mathrm{C} 25-\mathrm{C} 43-\mathrm{C} 44$ & $-63.2(3)$ \\
\hline C21-C22-C23-C24 & $-0.3(4)$ & $\mathrm{C} 24-\mathrm{C} 25-\mathrm{C} 43-\mathrm{C} 46$ & $-121.2(3)$ \\
\hline $\mathrm{C} 21-\mathrm{C} 22-\mathrm{C} 23-\mathrm{C} 47$ & $-180.0(2)$ & $\mathrm{C} 26-\mathrm{C} 25-\mathrm{C} 43-\mathrm{C} 46$ & $58.8(3)$ \\
\hline $\mathrm{C} 22-\mathrm{C} 23-\mathrm{C} 24-\mathrm{C} 25$ & $0.5(4)$ & $\mathrm{C} 24-\mathrm{C} 25-\mathrm{C} 43-\mathrm{C} 45$ & $-3.3(4)$ \\
\hline C47-C23-C24-C25 & $-179.8(2)$ & $\mathrm{C} 26-\mathrm{C} 25-\mathrm{C} 43-\mathrm{C} 45$ & $176.7(2)$ \\
\hline $\mathrm{C} 23-\mathrm{C} 24-\mathrm{C} 25-\mathrm{C} 26$ & $0.1(4)$ & $\mathrm{C} 22-\mathrm{C} 23-\mathrm{C} 47-\mathrm{C} 50$ & $3.3(4)$ \\
\hline $\mathrm{C} 23-\mathrm{C} 24-\mathrm{C} 25-\mathrm{C} 43$ & $-180.0(2)$ & $\mathrm{C} 24-\mathrm{C} 23-\mathrm{C} 47-\mathrm{C} 50$ & $-176.4(3)$ \\
\hline $\mathrm{C} 22-\mathrm{C} 21-\mathrm{C} 26-\mathrm{C} 25$ & $1.2(4)$ & $\mathrm{C} 22-\mathrm{C} 23-\mathrm{C} 47-\mathrm{C} 48$ & $-117.4(3)$ \\
\hline $\mathrm{P} 2-\mathrm{C} 21-\mathrm{C} 26-\mathrm{C} 25$ & $-176.6(2)$ & $\mathrm{C} 24-\mathrm{C} 23-\mathrm{C} 47-\mathrm{C} 48$ & $62.9(3)$ \\
\hline $\mathrm{C} 24-\mathrm{C} 25-\mathrm{C} 26-\mathrm{C} 21$ & $-1.0(4)$ & $\mathrm{C} 22-\mathrm{C} 23-\mathrm{C} 47-\mathrm{C} 49$ & $122.9(3)$ \\
\hline $\mathrm{C} 43-\mathrm{C} 25-\mathrm{C} 26-\mathrm{C} 21$ & $179.1(2)$ & $\mathrm{C} 24-\mathrm{C} 23-\mathrm{C} 47-\mathrm{C} 49$ & $-56.8(3)$ \\
\hline C6-C7-C27-C28 & $-8.2(4)$ & $\mathrm{C} 20-\mathrm{C} 19-\mathrm{C} 51-\mathrm{C} 53$ & $1.0(4)$ \\
\hline C8-C7-C27-C28 & $173.6(3)$ & C18-C19-C51-C53 & $-178.4(2)$ \\
\hline C6-C7-C27-C29 & $113.2(3)$ & C20-C19-C51-C52 & $-119.5(3)$ \\
\hline C8-C7-C27-C29 & $-65.0(3)$ & C18-C19-C51-C52 & $61.2(3)$ \\
\hline C6-C7-C27-C30 & $-128.1(3)$ & C20-C19-C51-C54 & $120.6(3)$ \\
\hline C8-C7-C27-C30 & $53.8(3)$ & C18-C19-C51-C54 & $-58.8(3)$ \\
\hline C4-C5-C31-C32 & $-172.9(3)$ & $\mathrm{C} 16-\mathrm{C} 17-\mathrm{C} 55-\mathrm{C} 57$ & $-60.4(4)$ \\
\hline C6-C5-C31-C32 & $7.6(4)$ & C18-C17-C55-C57 & 120.1(4) \\
\hline C4-C5-C31-C34 & $66.7(3)$ & C55'-C17-C55-C57 & $176(5)$ \\
\hline C6-C5-C31-C34 & $-112.7(3)$ & C16-C17-C55-C58 & 178.9(3) \\
\hline C4-C5-C31-C33 & $-52.9(3)$ & C18-C17-C55-C58 & $-0.6(5)$ \\
\hline C6-C5-C31-C33 & $127.7(3)$ & C55'-C17-C55-C58 & $55(4)$ \\
\hline C12-C11-C35-C38 & $-106.0(3)$ & C16-C17-C55-C56 & $60.4(4)$ \\
\hline C10-C11-C35-C38 & $72.3(3)$ & C18-C17-C55-C56 & $-119.1(4)$ \\
\hline C12-C11-C35-C37 & $134.5(3)$ & C55'-C17-C55-C56 & $-64(4)$ \\
\hline C10-C11-C35-C37 & $-47.2(4)$ & C16-C17-C55'-C57' & $-61(2)$ \\
\hline C12-C11-C35-C36 & $14.3(4)$ & C18-C17-C55'-C57' & $138(2)$ \\
\hline C10-C11-C35-C36 & $-167.4(3)$ & C55-C17-C55'-C57' & $5(4)$ \\
\hline C14-C13-C39-C40 & $2.7(4)$ & C16-C17-C55'-C58' & $-179.6(19)$ \\
\hline C12-C13-C39-C40 & $-176.3(3)$ & C18-C17-C55'-C58' & $19(3)$ \\
\hline C14-C13-C39-C41 & $122.4(3)$ & C55-C17-C55'-C58' & $-114(5)$ \\
\hline C12-C13-C39-C41 & $-56.5(3)$ & C16-C17-C55'-C56' & $58(3)$ \\
\hline C14-C13-C39-C42 & $-118.2(3)$ & $\mathrm{C} 18-\mathrm{C} 17-\mathrm{C} 55^{\prime}-\mathrm{C} 56^{\prime}$ & $-104(2)$ \\
\hline
\end{tabular}


C55-C17-C55'-C56'

C62-O1-C59-C60

O1-C59-C60-O2

C59-C60-O2-C61

C60-O2-C61-C62

C59-O1-C62-C61

O2-C61-C62-O1
124(6)

58.2(4)

$-59.0(5)$

$57.4(5)$

$-57.4(5)$

$-57.7(4)$

58.6(4)
C66-O3-C63-C64

57.3(4)

O3-C63-C64-O4

$-57.1(4)$

C63-C64-O4-C65

56.5(4)

C64-O4-C65-C66

$-58.0(4)$

C63-O3-C66-C65

$-58.7(4)$

O4-C65-C66-O3

60.3(4) 


\section{3 $\mathrm{FeCl}_{2}(\mathrm{dpbz})$}

REFERENCE NUMBER: neijk31

CRYSTAL STRUCTURE REPORT

$$
\mathrm{C}_{30} \mathrm{H}_{24} \mathrm{Cl}_{2} \mathrm{Fe} \mathrm{P}_{2}
$$

or

$(\mathrm{dpbz}) \mathrm{FeCl}_{2} \cdot \mathrm{xSolvent}$

Report prepared for:

J. Bailey, J. Kneebone, Prof. M. Neidig

September 28, 2014

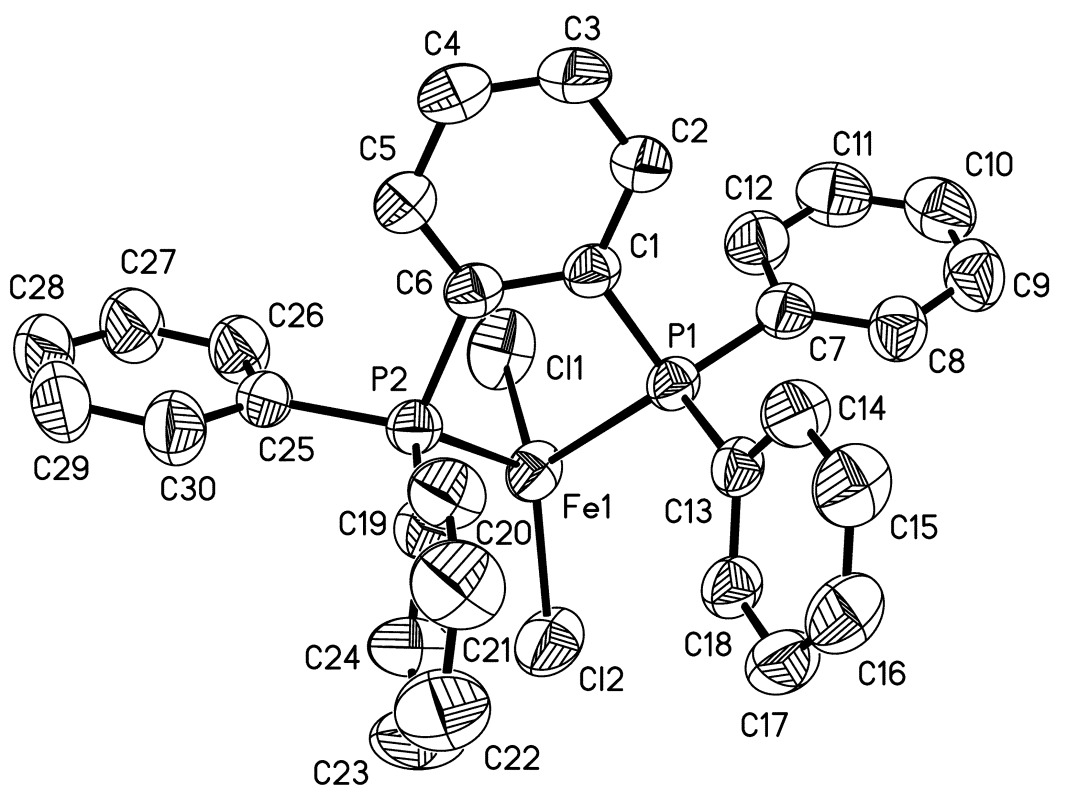

William W. Brennessel

X-ray Crystallographic Facility

Department of Chemistry, University of Rochester

120 Trustee Road

Rochester, NY 14627 


\section{Data collection}

A crystal $\left(0.24 \times 0.20 \times 0.12 \mathrm{~mm}^{3}\right)$ was placed onto the tip of a thin glass optical fiber and mounted on a Bruker SMART APEX II CCD platform diffractometer for a data collection at $173(2) \mathrm{K} .{ }^{1}$ A preliminary set of cell constants and an orientation matrix were calculated from reflections harvested from three orthogonal wedges of reciprocal space. The full data collection was carried out using MoKa radiation (graphite monochromator) with a frame time of 45 seconds and a detector distance of $4.02 \mathrm{~cm}$. A randomly oriented region of reciprocal space was surveyed: six major sections of frames were collected with $0.50^{\circ}$ steps in $\omega$ at six different $\phi$ settings and a detector position of $-38^{\circ}$ in $2 \theta$. The intensity data were corrected for absorption. ${ }^{2}$ Final cell constants were calculated from the xyz centroids of 4091 strong reflections from the actual data collection after integration. ${ }^{3}$ See Table 1 for additional crystal and refinement information.

Structure solution and refinement

The structure was solved using SIR2011 4 and refined using SHELXL-2014/7.5 The space group $P$-1 was determined based on intensity statistics. A direct-methods solution was calculated which provided most nonhydrogen atoms from the E-map. Full-matrix least squares / difference Fourier cycles were performed which located the remaining non-hydrogen atoms. All non-hydrogen atoms were refined with anisotropic displacement parameters. All hydrogen atoms were placed in ideal positions and refined as riding atoms with relative isotropic displacement parameters.

Highly disordered solvent (perhaps toluene) was found in a pocket located at a crystallographic inversion center. Reflection contributions from the disordered solvent were fixed and added to the calculated structure factors using the SQUEEZE routine of program Platon, ${ }^{6}$ which determined there to be 59 electrons in $205 \AA^{3}$ that were accounted for per unit cell. Because the exact identity and amount of disordered solvent is not known, no disordered solvent was included in the atom list and molecular formula. Thus all calculations that derive from the molecular formula (e.g., F(000), density, molecular weight, etc.) are known to be incorrect.

The final full matrix least squares refinement converged to $R 1=0.0405\left(F^{2}, I>2 \sigma(I)\right)$ and $w R 2=0.1096\left(F^{2}\right.$, all data).

\section{Structure description}

The structure is the one suggested. The asymmetric unit contains one iron complex in a general position and onehalf of a solvent molecule (see above) on a crystallographic inversion center.

Unless noted otherwise all structural diagrams containing thermal displacement ellipsoids are drawn at the $50 \%$ probability level. 
Data collection, structure solution, and structure refinement were conducted at the X-ray Crystallographic Facility, B51 Hutchison Hall, Department of Chemistry, University of Rochester. All publications arising from this report MUST either 1) include William W. Brennessel as a coauthor or 2) acknowledge William W. Brennessel and the Xray Crystallographic Facility of the Department of Chemistry at the University of Rochester.

1 APEX3, version 2015.5-2; Bruker AXS: Madison, WI, 2015.

2 Sheldrick, G. M. SADABS, version 2014/5; J. Appl. Cryst. 2015, 48, 3-10.

3 SAINT, version 8.34A; Bruker AXS: Madison, WI, 2013.

4 Burla, M. C.; Caliandro, R.; Camalli, M.; Carrozzini, B.; Cascarano, G. L.; Giacovazzo, C.; Mallamo, M.;

Mazzone, A.; Polidori, G.; Spagna, R. SIR2011, version 1.0; J. Appl. Cryst. 2012, 45, 357-361.

5 Sheldrick, G. M. SHELXL-2014/7; Acta. Cryst. 2015, C71, 3-8.

6 Spek, A. L. PLATON, version 07052015; Acta. Cryst. 2015, C71, 9-18.

Some equations of interest:

$$
\begin{gathered}
R_{\text {int }}=\Sigma\left|F_{\mathrm{o}}^{2}-<F_{\mathrm{o}}^{2}>\right| / \Sigma\left|F_{\mathrm{o}}{ }^{2}\right| \\
R 1=\Sigma|| F_{\mathrm{o}}|-| F_{\mathrm{c}}|| / \Sigma\left|F_{\mathrm{o}}\right| \\
w R 2=\left[\Sigma\left[w\left(F_{\mathrm{o}}{ }^{2}-F_{\mathrm{c}}{ }^{2}\right)^{2}\right] / \Sigma\left[w\left(F_{\mathrm{o}}^{2}\right)^{2}\right]\right]^{1 / 2} \\
\text { where } w=1 /\left[\sigma^{2}\left(F_{\mathrm{o}}{ }^{2}\right)+(a P)^{2}+b P\right] \text { and } \\
P=1 / 3 \max \left(0, F_{\mathrm{o}}^{2}\right)+2 / 3 F_{\mathrm{c}}^{2} \\
\text { GOF }=S=\left[\Sigma\left[w\left(F_{\mathrm{o}}{ }^{2}-F_{\mathrm{c}}{ }^{2}\right)^{2}\right] /(m-n)\right]^{1 / 2}
\end{gathered}
$$

where $m=$ number of reflections and $n=$ number of parameters

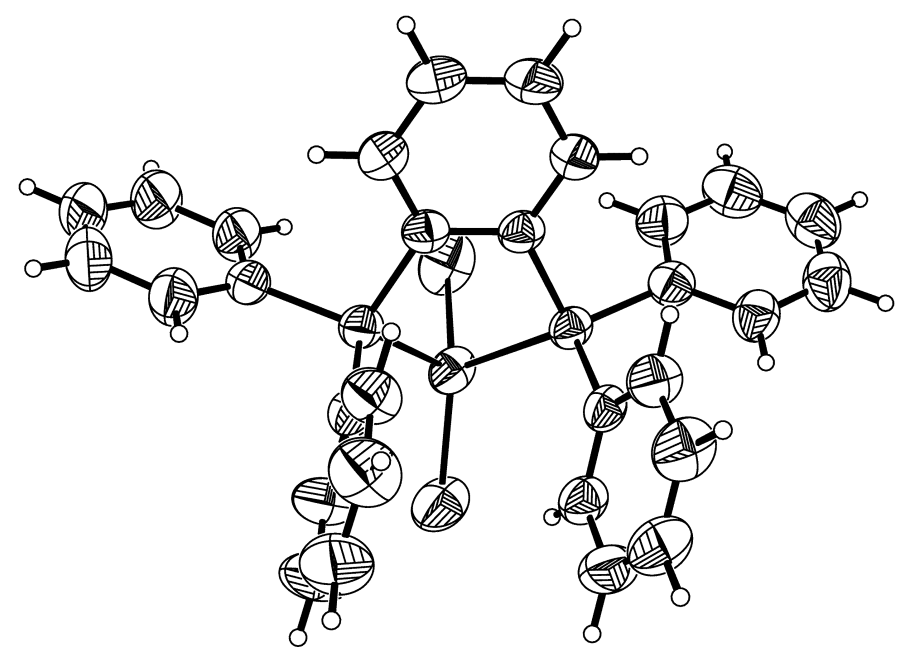


Table S13. Crystal data and structure refinement for neijk31.

\begin{tabular}{|c|c|c|}
\hline Identification code & \multicolumn{2}{|l|}{ neijk31 } \\
\hline Empirical formula & \multicolumn{2}{|c|}{$\mathrm{C} 30 \mathrm{H} 24 \mathrm{Cl} 2 \mathrm{Fe} \mathrm{P} 2$} \\
\hline Formula weight & \multicolumn{2}{|l|}{573.18} \\
\hline Temperature & \multicolumn{2}{|l|}{$173(2) \mathrm{K}$} \\
\hline Wavelength & \multicolumn{2}{|l|}{$0.71073 \AA$} \\
\hline Crystal system & \multicolumn{2}{|l|}{ triclinic } \\
\hline Space group & \multicolumn{2}{|l|}{$P-1$} \\
\hline \multirow[t]{3}{*}{ Unit cell dimensions } & $a=9.381(2) \AA$ & $\alpha=82.096(5)^{\circ}$ \\
\hline & $b=10.138(2) \AA$ & $\beta=81.479(5)^{\circ}$ \\
\hline & $c=17.591(4) \AA$ & $\gamma=68.716(5)^{\circ}$ \\
\hline Volume & \multicolumn{2}{|l|}{$1535.4(6) \AA^{3}$} \\
\hline$Z$ & \multicolumn{2}{|l|}{2} \\
\hline Density (calculated) & \multicolumn{2}{|l|}{$1.240 \mathrm{Mg} / \mathrm{m}^{3}$} \\
\hline Absorption coefficient & \multicolumn{2}{|l|}{$0.785 \mathrm{~mm}^{-1}$} \\
\hline$F(000)$ & \multicolumn{2}{|l|}{588} \\
\hline Crystal color, morphology & \multicolumn{2}{|l|}{ colorless, block } \\
\hline Crystal size & \multicolumn{2}{|c|}{$0.24 \times 0.20 \times 0.12 \mathrm{~mm}^{3}$} \\
\hline Theta range for data collection & \multicolumn{2}{|l|}{2.343 to $28.348^{\circ}$} \\
\hline Index ranges & \multicolumn{2}{|c|}{$-12 \leq h \leq 12,-13 \leq k \leq 13,-23 \leq l \leq 23$} \\
\hline Reflections collected & \multicolumn{2}{|l|}{32606} \\
\hline Independent reflections & \multicolumn{2}{|c|}{$7663[R($ int $)=0.0433]$} \\
\hline Observed reflections & \multicolumn{2}{|l|}{5295} \\
\hline Completeness to theta $=28.283^{\circ}$ & \multicolumn{2}{|l|}{$99.9 \%$} \\
\hline Absorption correction & \multicolumn{2}{|l|}{ Multi-scan } \\
\hline Max. and min. transmission & \multicolumn{2}{|c|}{0.7457 and 0.6560} \\
\hline Refinement method & \multicolumn{2}{|c|}{ Full-matrix least-squares on $F^{2}$} \\
\hline Data / restraints / parameters & \multicolumn{2}{|l|}{$7663 / 0 / 316$} \\
\hline Goodness-of-fit on $F^{2}$ & \multicolumn{2}{|l|}{1.012} \\
\hline Final $R$ indices $[I>2 \operatorname{sigma}(I)]$ & \multicolumn{2}{|c|}{$R 1=0.0405, w R 2=0.0969$} \\
\hline$R$ indices (all data) & \multicolumn{2}{|c|}{$R 1=0.0657, w R 2=0.1096$} \\
\hline Largest diff. peak and hole & \multicolumn{2}{|c|}{0.469 and -0.289 e. $\AA^{-3}$} \\
\hline
\end{tabular}


Table S14. Atomic coordinates $\left(\times 10^{4}\right)$ and equivalent isotropic displacement parameters $\left(\AA^{2} \times 10^{3}\right)$ for neijk31. $U_{e q}$ is defined as one third of the trace of the orthogonalized $U_{i j}$ tensor.

\begin{tabular}{|c|c|c|c|c|}
\hline & $\mathrm{x}$ & $\mathrm{y}$ & $\mathrm{z}$ & $\mathrm{U}_{\mathrm{eq}}$ \\
\hline $\mathrm{Fe} 1$ & $-969(1)$ & $7580(1)$ & $2110(1)$ & $53(1)$ \\
\hline Cl1 & $-2145(1)$ & $9477(1)$ & $1342(1)$ & $82(1)$ \\
\hline $\mathrm{Cl} 2$ & $-2126(1)$ & $6257(1)$ & $2870(1)$ & $90(1)$ \\
\hline P1 & $1417(1)$ & $6412(1)$ & $1320(1)$ & $43(1)$ \\
\hline $\mathrm{P} 2$ & $808(1)$ & $8251(1)$ & $2714(1)$ & $45(1)$ \\
\hline $\mathrm{C} 1$ & 2439(2) & $7655(2)$ & 1271(1) & $44(1)$ \\
\hline $\mathrm{C} 2$ & $3465(3)$ & $7816(2)$ & $643(1)$ & $58(1)$ \\
\hline C3 & $4247(3)$ & 8731(3) & 641(2) & $70(1)$ \\
\hline $\mathrm{C} 4$ & $3999(3)$ & $9524(3)$ & $1255(2)$ & $69(1)$ \\
\hline C5 & 2965(3) & 9395(2) & 1879(1) & $56(1)$ \\
\hline C6 & $2179(2)$ & $8464(2)$ & $1900(1)$ & $44(1)$ \\
\hline C7 & $1395(2)$ & 6181(2) & $317(1)$ & $50(1)$ \\
\hline $\mathrm{C} 8$ & 2099(3) & 4881(3) & $9(1)$ & $58(1)$ \\
\hline C9 & 1977(3) & 4773(3) & $-754(2)$ & $72(1)$ \\
\hline $\mathrm{C} 10$ & 1164(3) & 5919(4) & $-1201(2)$ & $76(1)$ \\
\hline C11 & $445(3)$ & 7204(3) & $-901(2)$ & $80(1)$ \\
\hline $\mathrm{C} 12$ & $557(3)$ & 7344(3) & $-144(2)$ & $68(1)$ \\
\hline C13 & 2732(2) & $4755(2)$ & 1733(1) & $44(1)$ \\
\hline $\mathrm{C} 14$ & $4294(2)$ & $4268(2)$ & $1480(1)$ & $58(1)$ \\
\hline C15 & $5255(3)$ & 3008(3) & $1804(2)$ & $71(1)$ \\
\hline $\mathrm{C} 16$ & $4695(3)$ & 2239(3) & $2387(2)$ & $73(1)$ \\
\hline C17 & $3158(3)$ & 2712(3) & $2653(2)$ & $68(1)$ \\
\hline $\mathrm{C} 18$ & $2176(3)$ & $3967(2)$ & 2321(1) & $54(1)$ \\
\hline C19 & 1939(3) & $6869(2)$ & $3380(1)$ & $50(1)$ \\
\hline $\mathrm{C} 20$ & $3524(3)$ & $6395(3)$ & $3313(1)$ & $64(1)$ \\
\hline $\mathrm{C} 21$ & $4316(4)$ & $5312(3)$ & $3822(2)$ & $86(1)$ \\
\hline $\mathrm{C} 22$ & $3554(4)$ & 4706(3) & $4395(2)$ & $93(1)$ \\
\hline $\mathrm{C} 23$ & $1985(4)$ & $5160(3)$ & $4471(2)$ & $92(1)$ \\
\hline $\mathrm{C} 24$ & $1168(3)$ & $6244(3)$ & $3960(2)$ & $71(1)$ \\
\hline $\mathrm{C} 25$ & $202(3)$ & 9891(2) & $3180(1)$ & $51(1)$ \\
\hline C26 & $-929(3)$ & $11056(3)$ & 2871(1) & $68(1)$ \\
\hline
\end{tabular}




\begin{tabular}{lrlll}
$\mathrm{C} 27$ & $-1419(4)$ & $12339(3)$ & $3191(2)$ & $85(1)$ \\
$\mathrm{C} 28$ & $-798(4)$ & $12448(3)$ & $3824(2)$ & $89(1)$ \\
$\mathrm{C} 29$ & $309(4)$ & $11304(3)$ & $4141(2)$ & $87(1)$ \\
$\mathrm{C} 30$ & $820(3)$ & $10009(3)$ & $3823(1)$ & $70(1)$ \\
\hline
\end{tabular}


Table S15. Bond lengths $[\AA]$ and angles $\left[{ }^{\circ}\right]$ for neijk31.

\begin{tabular}{|c|c|c|c|}
\hline $\mathrm{Fe}(1)-\mathrm{Cl}(2)$ & $2.2132(8)$ & $\mathrm{C}(14)-\mathrm{H}(14)$ & 0.9500 \\
\hline $\mathrm{Fe}(1)-\mathrm{Cl}(1)$ & $2.2190(8)$ & $C(15)-C(16)$ & $1.362(4)$ \\
\hline $\mathrm{Fe}(1)-\mathrm{P}(2)$ & $2.4330(7)$ & $\mathrm{C}(15)-\mathrm{H}(15)$ & 0.9500 \\
\hline $\mathrm{Fe}(1)-\mathrm{P}(1)$ & $2.4389(7)$ & $C(16)-C(17)$ & $1.374(4)$ \\
\hline $\mathrm{P}(1)-\mathrm{C}(13)$ & $1.816(2)$ & $\mathrm{C}(16)-\mathrm{H}(16)$ & 0.9500 \\
\hline $\mathrm{P}(1)-\mathrm{C}(7)$ & $1.816(2)$ & $\mathrm{C}(17)-\mathrm{C}(18)$ & $1.382(3)$ \\
\hline $\mathrm{P}(1)-\mathrm{C}(1)$ & $1.828(2)$ & $\mathrm{C}(17)-\mathrm{H}(17)$ & 0.9500 \\
\hline $\mathrm{P}(2)-\mathrm{C}(19)$ & $1.816(2)$ & $\mathrm{C}(18)-\mathrm{H}(18)$ & 0.9500 \\
\hline $\mathrm{P}(2)-\mathrm{C}(25)$ & $1.819(2)$ & $\mathrm{C}(19)-\mathrm{C}(20)$ & $1.379(3)$ \\
\hline $\mathrm{P}(2)-\mathrm{C}(6)$ & $1.822(2)$ & $\mathrm{C}(19)-\mathrm{C}(24)$ & $1.380(3)$ \\
\hline $\mathrm{C}(1)-\mathrm{C}(2)$ & $1.387(3)$ & $\mathrm{C}(20)-\mathrm{C}(21)$ & $1.377(3)$ \\
\hline$C(1)-C(6)$ & $1.407(3)$ & $\mathrm{C}(20)-\mathrm{H}(20)$ & 0.9500 \\
\hline $\mathrm{C}(2)-\mathrm{C}(3)$ & $1.376(3)$ & $\mathrm{C}(21)-\mathrm{C}(22)$ & $1.355(4)$ \\
\hline $\mathrm{C}(2)-\mathrm{H}(2)$ & 0.9500 & $\mathrm{C}(21)-\mathrm{H}(21)$ & 0.9500 \\
\hline $\mathrm{C}(3)-\mathrm{C}(4)$ & $1.376(4)$ & $\mathrm{C}(22)-\mathrm{C}(23)$ & $1.366(5)$ \\
\hline $\mathrm{C}(3)-\mathrm{H}(3)$ & 0.9500 & $\mathrm{C}(22)-\mathrm{H}(22)$ & 0.9500 \\
\hline$C(4)-C(5)$ & $1.378(3)$ & $\mathrm{C}(23)-\mathrm{C}(24)$ & $1.388(4)$ \\
\hline $\mathrm{C}(4)-\mathrm{H}(4)$ & 0.9500 & $\mathrm{C}(23)-\mathrm{H}(23)$ & 0.9500 \\
\hline$C(5)-C(6)$ & $1.387(3)$ & $\mathrm{C}(24)-\mathrm{H}(24)$ & 0.9500 \\
\hline $\mathrm{C}(5)-\mathrm{H}(5)$ & 0.9500 & $C(25)-C(26)$ & $1.379(3)$ \\
\hline$C(7)-C(12)$ & $1.386(3)$ & $\mathrm{C}(25)-\mathrm{C}(30)$ & $1.382(3)$ \\
\hline$C(7)-C(8)$ & $1.388(3)$ & $C(26)-C(27)$ & $1.383(4)$ \\
\hline $\mathrm{C}(8)-\mathrm{C}(9)$ & $1.385(3)$ & $\mathrm{C}(26)-\mathrm{H}(26)$ & 0.9500 \\
\hline $\mathrm{C}(8)-\mathrm{H}(8)$ & 0.9500 & $\mathrm{C}(27)-\mathrm{C}(28)$ & $1.366(4)$ \\
\hline $\mathrm{C}(9)-\mathrm{C}(10)$ & $1.354(4)$ & $\mathrm{C}(27)-\mathrm{H}(27)$ & 0.9500 \\
\hline $\mathrm{C}(9)-\mathrm{H}(9)$ & 0.9500 & $\mathrm{C}(28)-\mathrm{C}(29)$ & $1.361(4)$ \\
\hline$C(10)-C(11)$ & $1.370(4)$ & $\mathrm{C}(28)-\mathrm{H}(28)$ & 0.9500 \\
\hline $\mathrm{C}(10)-\mathrm{H}(10)$ & 0.9500 & $\mathrm{C}(29)-\mathrm{C}(30)$ & $1.391(4)$ \\
\hline$C(11)-C(12)$ & $1.381(4)$ & $\mathrm{C}(29)-\mathrm{H}(29)$ & 0.9500 \\
\hline $\mathrm{C}(11)-\mathrm{H}(11)$ & 0.9500 & $\mathrm{C}(30)-\mathrm{H}(30)$ & 0.9500 \\
\hline $\mathrm{C}(12)-\mathrm{H}(12)$ & 0.9500 & $\mathrm{Cl}(2)-\mathrm{Fe}(1)-\mathrm{Cl}(1)$ & $124.85(4)$ \\
\hline$C(13)-C(18)$ & $1.377(3)$ & $\mathrm{Cl}(2)-\mathrm{Fe}(1)-\mathrm{P}(2)$ & $115.16(3)$ \\
\hline$C(13)-C(14)$ & $1.390(3)$ & $\mathrm{Cl}(1)-\mathrm{Fe}(1)-\mathrm{P}(2)$ & $105.32(3)$ \\
\hline$C(14)-C(15)$ & $1.373(3)$ & $\mathrm{Cl}(2)-\mathrm{Fe}(1)-\mathrm{P}(1)$ & 119.01(3) \\
\hline
\end{tabular}




\begin{tabular}{|c|c|c|c|}
\hline $\mathrm{Cl}(1)-\mathrm{Fe}(1)-\mathrm{P}(1)$ & $102.85(3)$ & $\mathrm{C}(9)-\mathrm{C}(8)-\mathrm{H}(8)$ & 120.1 \\
\hline $\mathrm{P}(2)-\mathrm{Fe}(1)-\mathrm{P}(1)$ & $80.38(3)$ & $\mathrm{C}(7)-\mathrm{C}(8)-\mathrm{H}(8)$ & 120.1 \\
\hline$C(13)-P(1)-C(7)$ & $106.69(10)$ & $\mathrm{C}(10)-\mathrm{C}(9)-\mathrm{C}(8)$ & $120.8(3)$ \\
\hline$C(13)-P(1)-C(1)$ & $103.67(9)$ & $\mathrm{C}(10)-\mathrm{C}(9)-\mathrm{H}(9)$ & 119.6 \\
\hline $\mathrm{C}(7)-\mathrm{P}(1)-\mathrm{C}(1)$ & $104.07(10)$ & $\mathrm{C}(8)-\mathrm{C}(9)-\mathrm{H}(9)$ & 119.6 \\
\hline $\mathrm{C}(13)-\mathrm{P}(1)-\mathrm{Fe}(1)$ & $117.38(7)$ & $C(9)-C(10)-C(11)$ & $120.1(3)$ \\
\hline $\mathrm{C}(7)-\mathrm{P}(1)-\mathrm{Fe}(1)$ & $120.57(7)$ & $\mathrm{C}(9)-\mathrm{C}(10)-\mathrm{H}(10)$ & 120.0 \\
\hline $\mathrm{C}(1)-\mathrm{P}(1)-\mathrm{Fe}(1)$ & $102.08(7)$ & $\mathrm{C}(11)-\mathrm{C}(10)-\mathrm{H}(10)$ & 120.0 \\
\hline $\mathrm{C}(19)-\mathrm{P}(2)-\mathrm{C}(25)$ & $105.84(10)$ & $\mathrm{C}(10)-\mathrm{C}(11)-\mathrm{C}(12)$ & $120.3(3)$ \\
\hline$C(19)-P(2)-C(6)$ & $105.37(10)$ & $\mathrm{C}(10)-\mathrm{C}(11)-\mathrm{H}(11)$ & 119.8 \\
\hline$C(25)-P(2)-C(6)$ & $104.69(10)$ & $\mathrm{C}(12)-\mathrm{C}(11)-\mathrm{H}(11)$ & 119.8 \\
\hline $\mathrm{C}(19)-\mathrm{P}(2)-\mathrm{Fe}(1)$ & $114.56(8)$ & $C(11)-C(12)-C(7)$ & $120.1(2)$ \\
\hline $\mathrm{C}(25)-\mathrm{P}(2)-\mathrm{Fe}(1)$ & $122.11(8)$ & $\mathrm{C}(11)-\mathrm{C}(12)-\mathrm{H}(12)$ & 120.0 \\
\hline $\mathrm{C}(6)-\mathrm{P}(2)-\mathrm{Fe}(1)$ & $102.56(7)$ & $\mathrm{C}(7)-\mathrm{C}(12)-\mathrm{H}(12)$ & 120.0 \\
\hline$C(2)-C(1)-C(6)$ & $119.3(2)$ & $\mathrm{C}(18)-\mathrm{C}(13)-\mathrm{C}(14)$ & $118.9(2)$ \\
\hline $\mathrm{C}(2)-\mathrm{C}(1)-\mathrm{P}(1)$ & $122.38(17)$ & $\mathrm{C}(18)-\mathrm{C}(13)-\mathrm{P}(1)$ & $119.33(15)$ \\
\hline $\mathrm{C}(6)-\mathrm{C}(1)-\mathrm{P}(1)$ & $118.32(15)$ & $\mathrm{C}(14)-\mathrm{C}(13)-\mathrm{P}(1)$ & $121.78(17)$ \\
\hline$C(3)-C(2)-C(1)$ & $120.4(2)$ & $\mathrm{C}(15)-\mathrm{C}(14)-\mathrm{C}(13)$ & $120.2(2)$ \\
\hline $\mathrm{C}(3)-\mathrm{C}(2)-\mathrm{H}(2)$ & 119.8 & $\mathrm{C}(15)-\mathrm{C}(14)-\mathrm{H}(14)$ & 119.9 \\
\hline $\mathrm{C}(1)-\mathrm{C}(2)-\mathrm{H}(2)$ & 119.8 & $\mathrm{C}(13)-\mathrm{C}(14)-\mathrm{H}(14)$ & 119.9 \\
\hline$C(4)-C(3)-C(2)$ & $120.6(2)$ & $\mathrm{C}(16)-\mathrm{C}(15)-\mathrm{C}(14)$ & $120.4(2)$ \\
\hline $\mathrm{C}(4)-\mathrm{C}(3)-\mathrm{H}(3)$ & 119.7 & $\mathrm{C}(16)-\mathrm{C}(15)-\mathrm{H}(15)$ & 119.8 \\
\hline $\mathrm{C}(2)-\mathrm{C}(3)-\mathrm{H}(3)$ & 119.7 & $\mathrm{C}(14)-\mathrm{C}(15)-\mathrm{H}(15)$ & 119.8 \\
\hline$C(3)-C(4)-C(5)$ & $119.8(2)$ & $\mathrm{C}(15)-\mathrm{C}(16)-\mathrm{C}(17)$ & $120.2(2)$ \\
\hline $\mathrm{C}(3)-\mathrm{C}(4)-\mathrm{H}(4)$ & 120.1 & $\mathrm{C}(15)-\mathrm{C}(16)-\mathrm{H}(16)$ & 119.9 \\
\hline $\mathrm{C}(5)-\mathrm{C}(4)-\mathrm{H}(4)$ & 120.1 & $\mathrm{C}(17)-\mathrm{C}(16)-\mathrm{H}(16)$ & 119.9 \\
\hline$C(4)-C(5)-C(6)$ & $120.8(2)$ & $\mathrm{C}(16)-\mathrm{C}(17)-\mathrm{C}(18)$ & $119.8(2)$ \\
\hline $\mathrm{C}(4)-\mathrm{C}(5)-\mathrm{H}(5)$ & 119.6 & $\mathrm{C}(16)-\mathrm{C}(17)-\mathrm{H}(17)$ & 120.1 \\
\hline $\mathrm{C}(6)-\mathrm{C}(5)-\mathrm{H}(5)$ & 119.6 & $\mathrm{C}(18)-\mathrm{C}(17)-\mathrm{H}(17)$ & 120.1 \\
\hline$C(5)-C(6)-C(1)$ & $119.11(19)$ & $\mathrm{C}(13)-\mathrm{C}(18)-\mathrm{C}(17)$ & $120.4(2)$ \\
\hline $\mathrm{C}(5)-\mathrm{C}(6)-\mathrm{P}(2)$ & $122.39(17)$ & $\mathrm{C}(13)-\mathrm{C}(18)-\mathrm{H}(18)$ & 119.8 \\
\hline $\mathrm{C}(1)-\mathrm{C}(6)-\mathrm{P}(2)$ & $118.49(15)$ & $\mathrm{C}(17)-\mathrm{C}(18)-\mathrm{H}(18)$ & 119.8 \\
\hline$C(12)-C(7)-C(8)$ & $119.0(2)$ & $\mathrm{C}(20)-\mathrm{C}(19)-\mathrm{C}(24)$ & $119.2(2)$ \\
\hline$C(12)-C(7)-P(1)$ & $117.91(17)$ & $\mathrm{C}(20)-\mathrm{C}(19)-\mathrm{P}(2)$ & $122.83(18)$ \\
\hline $\mathrm{C}(8)-\mathrm{C}(7)-\mathrm{P}(1)$ & $122.99(18)$ & $\mathrm{C}(24)-\mathrm{C}(19)-\mathrm{P}(2)$ & $117.99(18)$ \\
\hline $\mathrm{C}(9)-\mathrm{C}(8)-\mathrm{C}(7)$ & $119.8(2)$ & $\mathrm{C}(21)-\mathrm{C}(20)-\mathrm{C}(19)$ & $120.0(3)$ \\
\hline
\end{tabular}




$\begin{array}{llll}\mathrm{C}(21)-\mathrm{C}(20)-\mathrm{H}(20) & 120.0 & \mathrm{C}(30)-\mathrm{C}(25)-\mathrm{P}(2) & 122.97(17) \\ \mathrm{C}(19)-\mathrm{C}(20)-\mathrm{H}(20) & 120.0 & \mathrm{C}(25)-\mathrm{C}(26)-\mathrm{C}(27) & 120.3(3) \\ \mathrm{C}(22)-\mathrm{C}(21)-\mathrm{C}(20) & 120.7(3) & \mathrm{C}(25)-\mathrm{C}(26)-\mathrm{H}(26) & 119.8 \\ \mathrm{C}(22)-\mathrm{C}(21)-\mathrm{H}(21) & 119.6 & \mathrm{C}(27)-\mathrm{C}(26)-\mathrm{H}(26) & 119.8 \\ \mathrm{C}(20)-\mathrm{C}(21)-\mathrm{H}(21) & 119.6 & \mathrm{C}(28)-\mathrm{C}(27)-\mathrm{C}(26) & 119.8(3) \\ \mathrm{C}(21)-\mathrm{C}(22)-\mathrm{C}(23) & 120.3(3) & \mathrm{C}(28)-\mathrm{C}(27)-\mathrm{H}(27) & 120.1 \\ \mathrm{C}(21)-\mathrm{C}(22)-\mathrm{H}(22) & 119.9 & \mathrm{C}(26)-\mathrm{C}(27)-\mathrm{H}(27) & 120.1 \\ \mathrm{C}(23)-\mathrm{C}(22)-\mathrm{H}(22) & 119.9 & \mathrm{C}(29)-\mathrm{C}(28)-\mathrm{C}(27) & 120.6(2) \\ \mathrm{C}(22)-\mathrm{C}(23)-\mathrm{C}(24) & 119.8(3) & \mathrm{C}(29)-\mathrm{C}(28)-\mathrm{H}(28) & 119.7 \\ \mathrm{C}(22)-\mathrm{C}(23)-\mathrm{H}(23) & 120.1 & \mathrm{C}(27)-\mathrm{C}(28)-\mathrm{H}(28) & 119.7 \\ \mathrm{C}(24)-\mathrm{C}(23)-\mathrm{H}(23) & 120.1 & \mathrm{C}(28)-\mathrm{C}(29)-\mathrm{C}(30) & 120.2(3) \\ \mathrm{C}(19)-\mathrm{C}(24)-\mathrm{C}(23) & 120.1(3) & \mathrm{C}(28)-\mathrm{C}(29)-\mathrm{H}(29) & 119.9 \\ \mathrm{C}(19)-\mathrm{C}(24)-\mathrm{H}(24) & 120.0 & \mathrm{C}(30)-\mathrm{C}(29)-\mathrm{H}(29) & 119.9 \\ \mathrm{C}(23)-\mathrm{C}(24)-\mathrm{H}(24) & 120.0 & \mathrm{C}(25)-\mathrm{C}(30)-\mathrm{C}(29) & 119.6(3) \\ \mathrm{C}(26)-\mathrm{C}(25)-\mathrm{C}(30) & 119.4(2) & \mathrm{C}(25)-\mathrm{C}(30)-\mathrm{H}(30) & 120.2 \\ \mathrm{C}(26)-\mathrm{C}(25)-\mathrm{P}(2) & 117.60(18) & \mathrm{C}(29)-\mathrm{C}(30)-\mathrm{H}(30) & 120.2\end{array}$


Table S16. Anisotropic displacement parameters $\left(\AA^{2} \times 10^{3}\right)$ for neijk31. The anisotropic displacement factor exponent takes the form: $-2 \pi^{2}\left[h^{2} a^{* 2} U_{11}+\ldots+2 h k a^{*} b^{*} U_{12}\right]$

\begin{tabular}{|c|c|c|c|c|c|c|}
\hline & $\mathrm{U}_{11}$ & $\mathrm{U}_{22}$ & $\mathrm{U}_{33}$ & $\mathrm{U}_{23}$ & $\mathrm{U}_{13}$ & $\mathrm{U}_{12}$ \\
\hline $\mathrm{Fe} 1$ & $36(1)$ & $45(1)$ & $74(1)$ & $-13(1)$ & $4(1)$ & $-11(1)$ \\
\hline Cl1 & $59(1)$ & $63(1)$ & $109(1)$ & $-12(1)$ & $-28(1)$ & $6(1)$ \\
\hline $\mathrm{Cl} 2$ & $75(1)$ & $74(1)$ & $124(1)$ & $-27(1)$ & $39(1)$ & $-41(1)$ \\
\hline P1 & $37(1)$ & $37(1)$ & 53(1) & $-6(1)$ & $1(1)$ & $-11(1)$ \\
\hline $\mathrm{P} 2$ & $43(1)$ & $39(1)$ & $48(1)$ & $-4(1)$ & $0(1)$ & $-11(1)$ \\
\hline $\mathrm{C} 1$ & $39(1)$ & $37(1)$ & $52(1)$ & $-2(1)$ & $0(1)$ & $-12(1)$ \\
\hline $\mathrm{C} 2$ & $64(1)$ & $54(1)$ & $57(1)$ & $-9(1)$ & 11(1) & $-27(1)$ \\
\hline $\mathrm{C} 3$ & $72(2)$ & $68(2)$ & $72(2)$ & $-5(1)$ & $18(1)$ & $-39(1)$ \\
\hline $\mathrm{C} 4$ & $76(2)$ & $69(2)$ & $76(2)$ & $-5(1)$ & $3(1)$ & $-47(1)$ \\
\hline $\mathrm{C} 5$ & $64(1)$ & $55(1)$ & $58(1)$ & $-7(1)$ & $-3(1)$ & $-30(1)$ \\
\hline C6 & $40(1)$ & $41(1)$ & $49(1)$ & $0(1)$ & $-5(1)$ & $-13(1)$ \\
\hline C7 & $44(1)$ & $52(1)$ & $57(1)$ & $-6(1)$ & $-1(1)$ & $-20(1)$ \\
\hline $\mathrm{C} 8$ & $54(1)$ & $58(1)$ & $64(1)$ & $-13(1)$ & $-6(1)$ & $-21(1)$ \\
\hline C9 & $70(2)$ & $82(2)$ & $73(2)$ & $-28(2)$ & $-1(1)$ & $-34(2)$ \\
\hline $\mathrm{C} 10$ & $80(2)$ & $106(2)$ & $58(2)$ & $-10(2)$ & $-8(1)$ & $-49(2)$ \\
\hline $\mathrm{C} 11$ & $82(2)$ & $91(2)$ & $65(2)$ & $9(2)$ & $-18(1)$ & $-32(2)$ \\
\hline $\mathrm{C} 12$ & $72(2)$ & $61(2)$ & $66(2)$ & $-1(1)$ & $-12(1)$ & $-18(1)$ \\
\hline $\mathrm{C} 13$ & $41(1)$ & $38(1)$ & $53(1)$ & $-10(1)$ & $-1(1)$ & $-11(1)$ \\
\hline $\mathrm{C} 14$ & $44(1)$ & $52(1)$ & $72(2)$ & $-5(1)$ & $2(1)$ & $-11(1)$ \\
\hline $\mathrm{C} 15$ & $42(1)$ & $59(2)$ & $97(2)$ & $-6(1)$ & $-4(1)$ & $-1(1)$ \\
\hline C16 & $65(2)$ & $47(1)$ & $92(2)$ & $2(1)$ & $-17(1)$ & $-2(1)$ \\
\hline $\mathrm{C} 17$ & $73(2)$ & $48(1)$ & $73(2)$ & $6(1)$ & $-5(1)$ & $-16(1)$ \\
\hline C18 & $48(1)$ & $44(1)$ & $64(1)$ & $-7(1)$ & $2(1)$ & $-13(1)$ \\
\hline C19 & $54(1)$ & $42(1)$ & $49(1)$ & $-6(1)$ & $-3(1)$ & $-12(1)$ \\
\hline $\mathrm{C} 20$ & $56(1)$ & $63(2)$ & $62(1)$ & $2(1)$ & $-6(1)$ & $-10(1)$ \\
\hline $\mathrm{C} 21$ & $70(2)$ & $80(2)$ & $85(2)$ & $6(2)$ & $-18(2)$ & $0(2)$ \\
\hline $\mathrm{C} 22$ & $107(3)$ & $70(2)$ & $82(2)$ & $19(2)$ & $-31(2)$ & $-9(2)$ \\
\hline $\mathrm{C} 23$ & $110(3)$ & $80(2)$ & $76(2)$ & $27(2)$ & $-9(2)$ & $-34(2)$ \\
\hline $\mathrm{C} 24$ & $67(2)$ & $69(2)$ & $68(2)$ & $14(1)$ & $0(1)$ & $-25(1)$ \\
\hline $\mathrm{C} 25$ & $58(1)$ & $40(1)$ & $49(1)$ & $-6(1)$ & $3(1)$ & $-13(1)$ \\
\hline C26 & $82(2)$ & $49(1)$ & $61(1)$ & $-6(1)$ & $-9(1)$ & $-6(1)$ \\
\hline
\end{tabular}




\begin{tabular}{lllllll}
$\mathrm{C} 27$ & $112(2)$ & $47(1)$ & $73(2)$ & $-9(1)$ & $-6(2)$ & $0(2)$ \\
$\mathrm{C} 28$ & $136(3)$ & $49(2)$ & $70(2)$ & $-19(1)$ & $10(2)$ & $-19(2)$ \\
$\mathrm{C} 29$ & $128(3)$ & $72(2)$ & $60(2)$ & $-20(1)$ & $-11(2)$ & $-28(2)$ \\
$\mathrm{C} 30$ & $89(2)$ & $56(1)$ & $61(1)$ & $-10(1)$ & $-13(1)$ & $-15(1)$ \\
\hline
\end{tabular}


Table S17. Hydrogen coordinates $\left(\times 10^{4}\right)$ and isotropic displacement parameters $\left(\AA^{2} \times 10^{3}\right)$ for neijk31.

\begin{tabular}{|c|c|c|c|c|}
\hline & $\mathrm{x}$ & $\mathrm{y}$ & z & $\mathrm{U}(\mathrm{eq})$ \\
\hline $\mathrm{H} 2$ & 3629 & 7290 & 211 & 70 \\
\hline H3 & 4965 & 8817 & 213 & 83 \\
\hline $\mathrm{H} 4$ & 4539 & 10158 & 1249 & 83 \\
\hline H5 & 2788 & 9951 & 2299 & 68 \\
\hline H8 & 2662 & 4068 & 319 & 69 \\
\hline H9 & 2470 & 3884 & -965 & 86 \\
\hline H10 & 1091 & 5831 & -1724 & 92 \\
\hline H11 & -133 & 8003 & -1215 & 95 \\
\hline H12 & 60 & 8239 & 60 & 81 \\
\hline H14 & 4698 & 4809 & 1081 & 70 \\
\hline H15 & 6317 & 2670 & 1619 & 85 \\
\hline H16 & 5369 & 1373 & 2611 & 87 \\
\hline H17 & 2771 & 2179 & 3063 & 81 \\
\hline H18 & 1111 & 4288 & 2501 & 64 \\
\hline $\mathrm{H} 20$ & 4069 & 6814 & 2915 & 77 \\
\hline $\mathrm{H} 21$ & 5408 & 4987 & 3772 & 103 \\
\hline $\mathrm{H} 22$ & 4115 & 3963 & 4744 & 112 \\
\hline $\mathrm{H} 23$ & 1454 & 4734 & 4873 & 110 \\
\hline $\mathrm{H} 24$ & 76 & 6557 & 4010 & 85 \\
\hline $\mathrm{H} 26$ & -1373 & 10976 & 2435 & 82 \\
\hline $\mathrm{H} 27$ & -2185 & 13144 & 2970 & 102 \\
\hline $\mathrm{H} 28$ & -1142 & 13329 & 4045 & 107 \\
\hline $\mathrm{H} 29$ & 733 & 11391 & 4582 & 104 \\
\hline H30 & 1590 & 9210 & 4045 & 84 \\
\hline
\end{tabular}


Table S18. Torsion angles $\left[{ }^{\circ}\right]$ for neijk 31 .

\begin{tabular}{|c|c|c|c|}
\hline C13-P1-C1-C2 & $87.52(19)$ & $\mathrm{C} 10-\mathrm{C} 11-\mathrm{C} 12-\mathrm{C} 7$ & $-0.2(4)$ \\
\hline C7-P1-C1-C2 & $-23.9(2)$ & C8-C7-C12-C11 & $-0.7(4)$ \\
\hline Fe1-P1-C1-C2 & $-150.06(17)$ & $\mathrm{P} 1-\mathrm{C} 7-\mathrm{C} 12-\mathrm{C} 11$ & $-176.9(2)$ \\
\hline C13-P1-C1-C6 & $-91.85(16)$ & C7-P1-C13-C18 & $-116.57(18)$ \\
\hline C7-P1-C1-C6 & 156.71(16) & C1-P1-C13-C18 & $133.92(18)$ \\
\hline Fe1-P1-C1-C6 & $30.57(16)$ & Fe1-P1-C13-C18 & $22.3(2)$ \\
\hline $\mathrm{C} 6-\mathrm{C} 1-\mathrm{C} 2-\mathrm{C} 3$ & $1.5(3)$ & C7-P1-C13-C14 & $64.5(2)$ \\
\hline $\mathrm{P} 1-\mathrm{C} 1-\mathrm{C} 2-\mathrm{C} 3$ & $-177.86(19)$ & C1-P1-C13-C14 & $-45.0(2)$ \\
\hline $\mathrm{C} 1-\mathrm{C} 2-\mathrm{C} 3-\mathrm{C} 4$ & $-1.4(4)$ & Fe1-P1-C13-C14 & $-156.64(16)$ \\
\hline $\mathrm{C} 2-\mathrm{C} 3-\mathrm{C} 4-\mathrm{C} 5$ & $0.4(4)$ & C18-C13-C14-C15 & $1.2(4)$ \\
\hline $\mathrm{C} 3-\mathrm{C} 4-\mathrm{C} 5-\mathrm{C} 6$ & $0.6(4)$ & P1-C13-C14-C15 & $-179.83(19)$ \\
\hline $\mathrm{C} 4-\mathrm{C} 5-\mathrm{C} 6-\mathrm{C} 1$ & $-0.6(3)$ & $\mathrm{C} 13-\mathrm{C} 14-\mathrm{C} 15-\mathrm{C} 16$ & $-1.5(4)$ \\
\hline C4-C5-C6-P2 & $179.68(19)$ & C14-C15-C16-C17 & $0.6(4)$ \\
\hline $\mathrm{C} 2-\mathrm{C} 1-\mathrm{C} 6-\mathrm{C} 5$ & $-0.5(3)$ & $\mathrm{C} 15-\mathrm{C} 16-\mathrm{C} 17-\mathrm{C} 18$ & $0.6(4)$ \\
\hline P1-C1-C6-C5 & $178.89(16)$ & C14-C13-C18-C17 & $-0.1(3)$ \\
\hline $\mathrm{C} 2-\mathrm{C} 1-\mathrm{C} 6-\mathrm{P} 2$ & $179.26(16)$ & $\mathrm{P} 1-\mathrm{C} 13-\mathrm{C} 18-\mathrm{C} 17$ & $-179.06(18)$ \\
\hline P1-C1-C6-P2 & $-1.3(2)$ & $\mathrm{C} 16-\mathrm{C} 17-\mathrm{C} 18-\mathrm{C} 13$ & $-0.8(4)$ \\
\hline C19-P2-C6-C5 & $-88.77(19)$ & $\mathrm{C} 25-\mathrm{P} 2-\mathrm{C} 19-\mathrm{C} 20$ & $-97.5(2)$ \\
\hline $\mathrm{C} 25-\mathrm{P} 2-\mathrm{C} 6-\mathrm{C} 5$ & $22.6(2)$ & C6-P2-C19-C20 & $13.0(2)$ \\
\hline Fe1-P2-C6-C5 & $151.04(17)$ & Fe1-P2-C19-C20 & $124.98(19)$ \\
\hline C19-P2-C6-C1 & $91.47(17)$ & C25-P2-C19-C24 & $84.6(2)$ \\
\hline C25-P2-C6-C1 & $-157.14(16)$ & C6-P2-C19-C24 & $-164.79(19)$ \\
\hline Fe1-P2-C6-C1 & $-28.72(16)$ & Fe1-P2-C19-C24 & $-52.9(2)$ \\
\hline C13-P1-C7-C12 & $-175.25(18)$ & C24-C19-C20-C21 & $-0.1(4)$ \\
\hline C1-P1-C7-C12 & $-66.0(2)$ & P2-C19-C20-C21 & $-178.0(2)$ \\
\hline $\mathrm{Fe} 1-\mathrm{P} 1-\mathrm{C} 7-\mathrm{C} 12$ & $47.5(2)$ & C19-C20-C21-C22 & $-0.2(5)$ \\
\hline $\mathrm{C} 13-\mathrm{P} 1-\mathrm{C} 7-\mathrm{C} 8$ & $8.7(2)$ & $\mathrm{C} 20-\mathrm{C} 21-\mathrm{C} 22-\mathrm{C} 23$ & $0.3(5)$ \\
\hline C1-P1-C7-C8 & 117.94(19) & $\mathrm{C} 21-\mathrm{C} 22-\mathrm{C} 23-\mathrm{C} 24$ & $0.1(5)$ \\
\hline Fe1-P1-C7-C8 & $-128.57(17)$ & $\mathrm{C} 20-\mathrm{C} 19-\mathrm{C} 24-\mathrm{C} 23$ & $0.5(4)$ \\
\hline C12-C7-C8-C9 & $1.2(3)$ & $\mathrm{P} 2-\mathrm{C} 19-\mathrm{C} 24-\mathrm{C} 23$ & $178.4(2)$ \\
\hline $\mathrm{P} 1-\mathrm{C} 7-\mathrm{C} 8-\mathrm{C} 9$ & $177.18(18)$ & $\mathrm{C} 22-\mathrm{C} 23-\mathrm{C} 24-\mathrm{C} 19$ & $-0.4(5)$ \\
\hline $\mathrm{C} 7-\mathrm{C} 8-\mathrm{C} 9-\mathrm{C} 10$ & $-0.8(4)$ & $\mathrm{C} 19-\mathrm{P} 2-\mathrm{C} 25-\mathrm{C} 26$ & $-167.13(19)$ \\
\hline C8-C9-C10-C11 & $-0.2(4)$ & $\mathrm{C} 6-\mathrm{P} 2-\mathrm{C} 25-\mathrm{C} 26$ & $81.8(2)$ \\
\hline C9-C10-C11-C12 & $0.7(5)$ & Fe1-P2-C25-C26 & $-33.6(2)$ \\
\hline
\end{tabular}




\begin{tabular}{lclr}
$\mathrm{C} 19-\mathrm{P} 2-\mathrm{C} 25-\mathrm{C} 30$ & $12.8(2)$ & $\mathrm{C} 26-\mathrm{C} 27-\mathrm{C} 28-\mathrm{C} 29$ & $0.5(5)$ \\
$\mathrm{C} 6-\mathrm{P} 2-\mathrm{C} 25-\mathrm{C} 30$ & $-98.3(2)$ & $\mathrm{C} 27-\mathrm{C} 28-\mathrm{C} 29-\mathrm{C} 30$ & $-0.1(5)$ \\
$\mathrm{Fe} 1-\mathrm{P} 2-\mathrm{C} 25-\mathrm{C} 30$ & $146.24(19)$ & $\mathrm{C} 26-\mathrm{C} 25-\mathrm{C} 30-\mathrm{C} 29$ & $-0.9(4)$ \\
$\mathrm{C} 30-\mathrm{C} 25-\mathrm{C} 26-\mathrm{C} 27$ & $1.3(4)$ & $\mathrm{P} 2-\mathrm{C} 25-\mathrm{C} 30-\mathrm{C} 29$ & $179.3(2)$ \\
$\mathrm{P} 2-\mathrm{C} 25-\mathrm{C} 26-\mathrm{C} 27$ & $-178.8(2)$ & $\mathrm{C} 28-\mathrm{C} 29-\mathrm{C} 30-\mathrm{C} 25$ & $0.2(5)$ \\
$\mathrm{C} 25-\mathrm{C} 26-\mathrm{C} 27-\mathrm{C} 28$ & $-1.1(5)$ & & \\
& & & \\
\hline
\end{tabular}




\section{4 $\mathrm{FeCl}_{2}$ (Xantphos)}

REFERENCE NUMBER: neivf02

CRYSTAL STRUCTURE REPORT

$\mathrm{C}_{39} \mathrm{H}_{32} \mathrm{Cl}_{2} \mathrm{Fe} \mathrm{O} \mathrm{P}_{2}$

or

$\mathrm{FeCl}_{2}$ (xantphos)

Report prepared for:

V. Fleischauer, Prof. M. Neidig

March 10, 2014

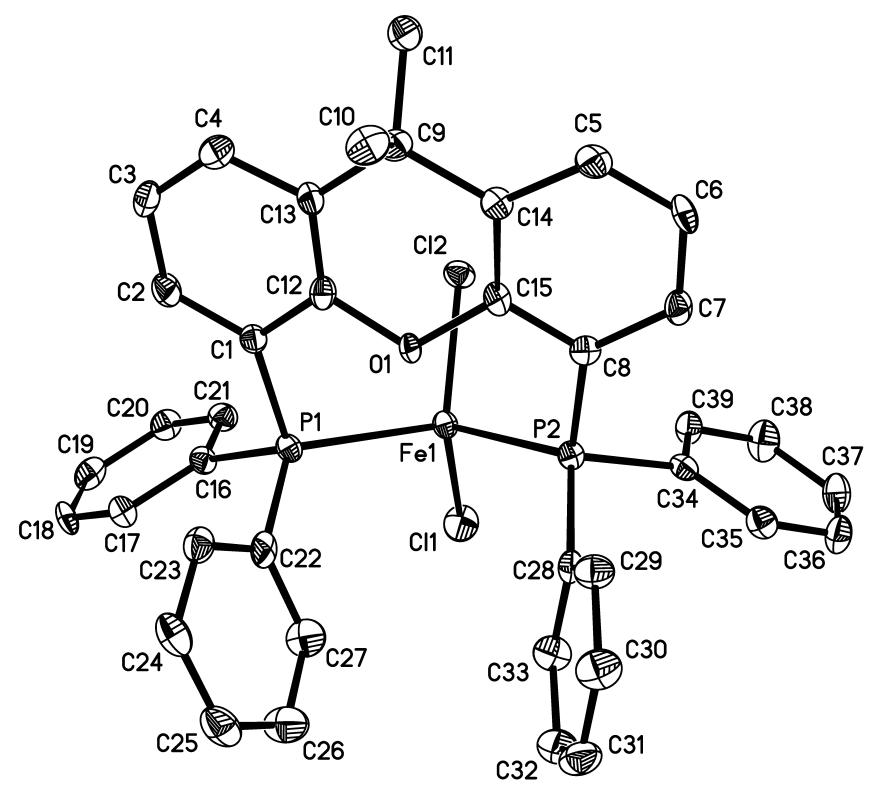

William W. Brennessel

X-ray Crystallographic Facility

Department of Chemistry, University of Rochester

120 Trustee Road

Rochester, NY 14627 


\section{Data collection}

A crystal $\left(0.26 \times 0.12 \times 0.06 \mathrm{~mm}^{3}\right)$ was placed onto the tip of a $0.1 \mathrm{~mm}$ diameter glass capillary tube or fiber and mounted on a Bruker SMART APEX II CCD platform diffractometer for a data collection at 100.0(5) $\mathrm{K}^{1} \mathrm{~A}$ preliminary set of cell constants and an orientation matrix were calculated from reflections harvested from three orthogonal wedges of reciprocal space. The full data collection was carried out using MoK $\alpha$ radiation (graphite monochromator) with a frame time of 90 seconds and a detector distance of $5.03 \mathrm{~cm}$. A randomly oriented region of reciprocal space was surveyed: six major sections of frames were collected with $0.50^{\circ}$ steps in $\omega$ at six different $\phi$ settings and a detector position of $-33^{\circ}$ in $2 \theta$. The intensity data were corrected for absorption. ${ }^{2}$ Final cell constants were calculated from the xyz centroids of 4070 strong reflections from the actual data collection after integration. ${ }^{3}$ See Table 1 for additional crystal and refinement information.

\section{Structure solution and refinement}

The structure was solved using SIR2011 4 and refined using SHELXL-2014. ${ }^{5}$ The space group $P 2{ }_{1} / n$ was determined based on systematic absences. A direct-methods solution was calculated which provided most nonhydrogen atoms from the E-map. Full-matrix least squares / difference Fourier cycles were performed which located the remaining non-hydrogen atoms. All non-hydrogen atoms were refined with anisotropic displacement parameters. All hydrogen atoms were placed in ideal positions and refined as riding atoms with relative isotropic displacement parameters. The final full matrix least squares refinement converged to $R 1=0.0581\left(F^{2}, I>2 \sigma(I)\right)$ and $w R 2=0.1388\left(F^{2}\right.$, all data).

\section{Structure description}

The structure is the one suggested. The asymmetric unit contains two independent molecules in general positions.

Unless noted otherwise all structural diagrams containing thermal displacement ellipsoids are drawn at the $50 \%$ probability level.

Data collection, structure solution, and structure refinement were conducted at the X-ray Crystallographic Facility, B51 Hutchison Hall, Department of Chemistry, University of Rochester. All publications arising from this report MUST either 1) include William W. Brennessel as a coauthor or 2) acknowledge William W. Brennessel and the Xray Crystallographic Facility of the Department of Chemistry at the University of Rochester. 
1 APEX2, version 2013.10-0; Bruker AXS: Madison, WI, 2013.

2 Sheldrick, G. M. SADABS, version 2012/1; University of Göttingen: Göttingen, Germany, 2012.

3 SAINT, version 8.34A; Bruker AXS: Madison, WI, 2013.

4 Burla, M. C.; Caliandro, R.; Camalli, M.; Carrozzini, B.; Cascarano, G. L.; Giacovazzo, C.; Mallamo, M.;

Mazzone, A.; Polidori, G.; Spagna, R. SIR2011: a new package for crystal structure determination and refinement, version 1.0; Istituto di Cristallografia: Bari, Italy, 2012.

5 Sheldrick, G. M. SHELXL-2014/1; University of Göttingen: Göttingen, Germany, 2014.

Some equations of interest:

$$
\begin{gathered}
R_{\mathrm{int}}=\Sigma\left|F_{\mathrm{o}}{ }^{2}-<F_{\mathrm{o}}{ }^{2}>\right| / \Sigma\left|F_{\mathrm{o}}{ }^{2}\right| \\
R 1=\Sigma|| F_{\mathrm{o}}|-| F_{\mathrm{c}}|/ \Sigma| F_{\mathrm{o}} \mid \\
w R 2=\left[\Sigma\left[w\left(F_{\mathrm{o}}{ }^{2}-F_{\mathrm{c}}{ }^{2}\right)^{2}\right] / \Sigma\left[w\left(F_{\mathrm{o}}{ }^{2}\right)^{2}\right]\right]^{1 / 2} \\
\text { where } w=1 /\left[\sigma^{2}\left(F_{\mathrm{o}}{ }^{2}\right)+(a P)^{2}+b P\right] \text { and } \\
P=1 / 3 \max \left(0, F_{\mathrm{o}}{ }^{2}\right)+2 / 3 F_{\mathrm{c}}{ }^{2} \\
\text { GOF }=S=\left[\Sigma\left[w\left(F_{\mathrm{o}}{ }^{2}-F_{\mathrm{c}}{ }^{2}\right)^{2}\right] /(m-n)\right]^{1 / 2}
\end{gathered}
$$

where $m=$ number of reflections and $n=$ number of parameters

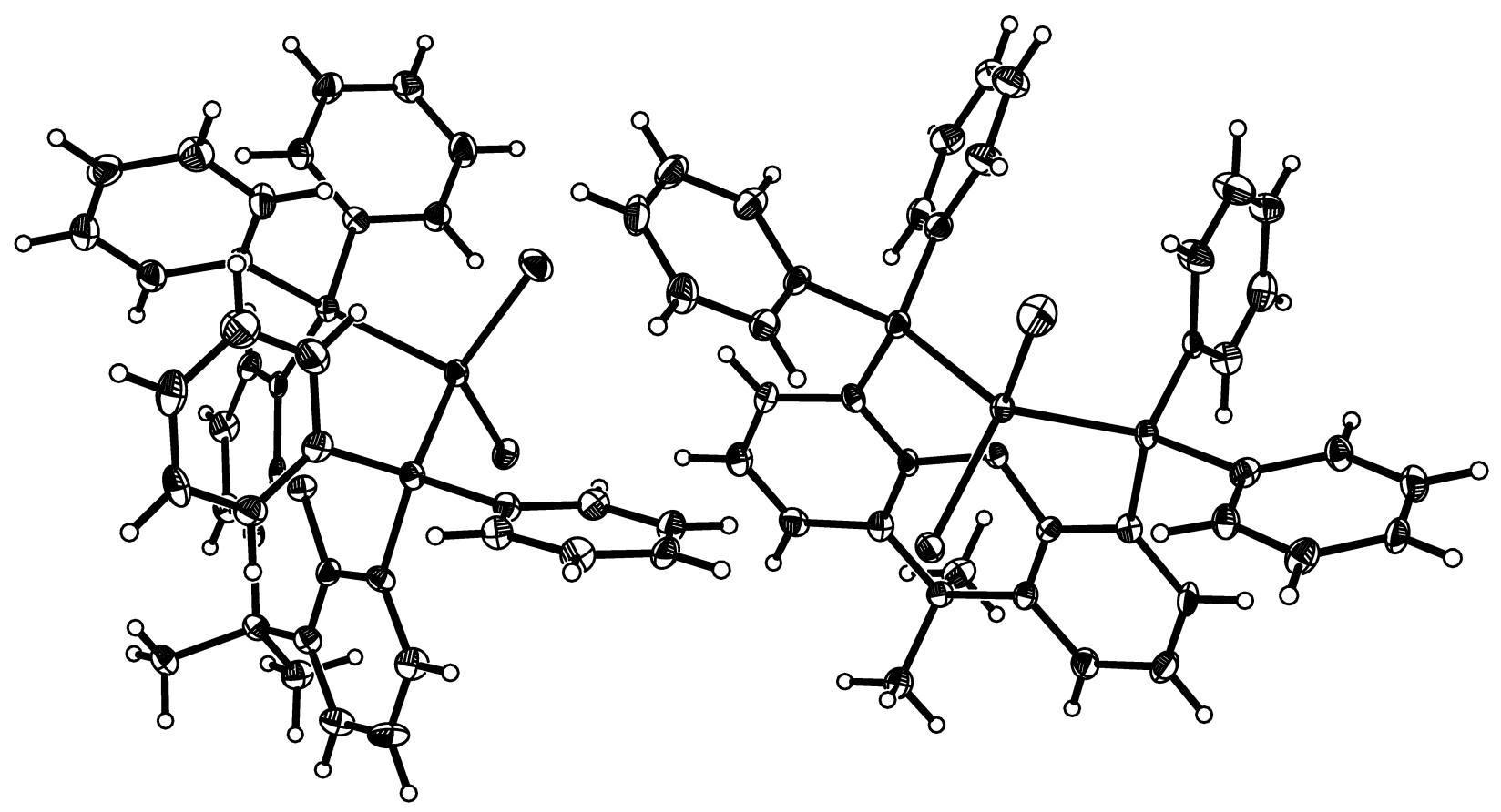



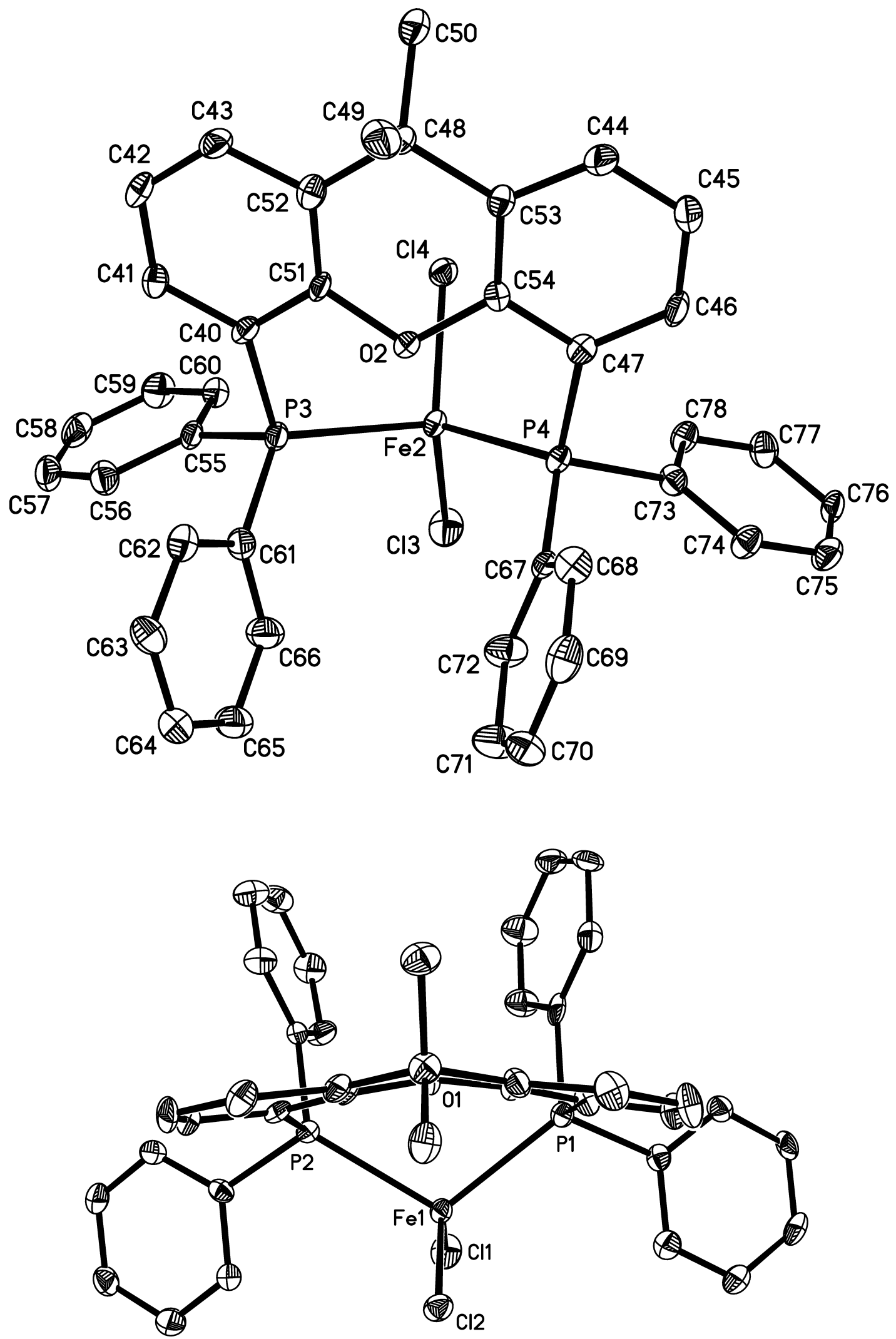
Table S19. Crystal data and structure refinement for neivf02.

\begin{tabular}{|c|c|c|}
\hline Identification code & \multicolumn{2}{|l|}{ neivf02 } \\
\hline Empirical formula & \multicolumn{2}{|c|}{$\mathrm{C} 39 \mathrm{H} 32 \mathrm{Cl} 2 \mathrm{Fe} \mathrm{O} \mathrm{P} 2$} \\
\hline Formula weight & \multicolumn{2}{|l|}{705.33} \\
\hline Temperature & \multicolumn{2}{|l|}{$100.0(5) \mathrm{K}$} \\
\hline Wavelength & \multicolumn{2}{|l|}{$0.71073 \AA$} \\
\hline Crystal system & \multicolumn{2}{|l|}{ monoclinic } \\
\hline Space group & \multicolumn{2}{|l|}{$P 2_{1} / n$} \\
\hline \multirow[t]{3}{*}{ Unit cell dimensions } & $a=16.882(4) \AA$ & $\alpha=90^{\circ}$ \\
\hline & $b=10.534(3) \AA$ & $\beta=93.412(4)^{\circ}$ \\
\hline & $c=37.753(9) \AA$ & $\gamma=90^{\circ}$ \\
\hline Volume & \multicolumn{2}{|l|}{$6702(3) \AA^{3}$} \\
\hline$Z$ & \multicolumn{2}{|l|}{8} \\
\hline Density (calculated) & \multicolumn{2}{|l|}{$1.398 \mathrm{Mg} / \mathrm{m}^{3}$} \\
\hline Absorption coefficient & \multicolumn{2}{|l|}{$0.736 \mathrm{~mm}^{-1}$} \\
\hline$F(000)$ & \multicolumn{2}{|l|}{2912} \\
\hline Crystal color, morphology & \multicolumn{2}{|l|}{ colorless, plate } \\
\hline Crystal size & \multicolumn{2}{|c|}{$0.26 \times 0.12 \times 0.06 \mathrm{~mm}^{3}$} \\
\hline Theta range for data collection & \multicolumn{2}{|l|}{1.963 to $27.519^{\circ}$} \\
\hline Index ranges & \multicolumn{2}{|c|}{$-21 \leq h \leq 21,-13 \leq k \leq 13,-49 \leq l \leq 49$} \\
\hline Reflections collected & \multicolumn{2}{|l|}{121316} \\
\hline Independent reflections & \multicolumn{2}{|c|}{$15389[R(\mathrm{int})=0.1290]$} \\
\hline Observed reflections & \multicolumn{2}{|l|}{8852} \\
\hline Completeness to theta $=27.485^{\circ}$ & \multicolumn{2}{|l|}{$100.0 \%$} \\
\hline Absorption correction & \multicolumn{2}{|l|}{ Multi-scan } \\
\hline Max. and min. transmission & \multicolumn{2}{|l|}{0.7456 and 0.6259} \\
\hline Refinement method & \multicolumn{2}{|c|}{ Full-matrix least-squares on $F^{2}$} \\
\hline Data / restraints / parameters & \multicolumn{2}{|l|}{$15389 / 0 / 815$} \\
\hline Goodness-of-fit on $F^{2}$ & \multicolumn{2}{|l|}{1.027} \\
\hline Final $R$ indices $[I>2 \operatorname{sigma}(I)]$ & \multicolumn{2}{|c|}{$R 1=0.0581, w R 2=0.1198$} \\
\hline$R$ indices (all data) & \multicolumn{2}{|c|}{$R 1=0.1202, w R 2=0.1388$} \\
\hline Largest diff. peak and hole & \multicolumn{2}{|c|}{0.901 and -0.467 e. $\AA^{-3}$} \\
\hline
\end{tabular}


Table S20. Atomic coordinates $\left(\times 10^{4}\right)$ and equivalent isotropic displacement parameters $\left(\AA^{2} \times 10^{3}\right)$ for neivf02. $U_{e q}$ is defined as one third of the trace of the orthogonalized $U_{i j}$ tensor.

\begin{tabular}{|c|c|c|c|c|}
\hline & $\mathrm{x}$ & $\mathrm{y}$ & $\mathrm{z}$ & $\mathrm{U}_{\mathrm{eq}}$ \\
\hline $\mathrm{Fe} 1$ & $3336(1)$ & $3368(1)$ & 1194(1) & $15(1)$ \\
\hline $\mathrm{Cl1}$ & $3986(1)$ & $2666(1)$ & $741(1)$ & $27(1)$ \\
\hline $\mathrm{Cl} 2$ & $3142(1)$ & 2153(1) & $1673(1)$ & $19(1)$ \\
\hline $\mathrm{P} 1$ & $3987(1)$ & $5322(1)$ & $1416(1)$ & $15(1)$ \\
\hline $\mathrm{P} 2$ & $1975(1)$ & $3862(1)$ & $970(1)$ & $14(1)$ \\
\hline $\mathrm{O} 1$ & $2407(1)$ & $5200(2)$ & $1616(1)$ & $15(1)$ \\
\hline $\mathrm{C} 1$ & $3685(2)$ & $5610(4)$ & $1862(1)$ & $16(1)$ \\
\hline $\mathrm{C} 2$ & $4200(2)$ & $5867(4)$ & $2156(1)$ & $22(1)$ \\
\hline $\mathrm{C} 3$ & $3910(3)$ & 5984(4) & $2490(1)$ & $26(1)$ \\
\hline $\mathrm{C} 4$ & $3108(3)$ & $5820(4)$ & $2536(1)$ & $24(1)$ \\
\hline $\mathrm{C} 5$ & $534(2)$ & 4189(4) & 1962(1) & $21(1)$ \\
\hline C6 & $174(2)$ & $3634(4)$ & $1659(1)$ & $21(1)$ \\
\hline $\mathrm{C} 7$ & $575(2)$ & $3567(4)$ & 1352(1) & $18(1)$ \\
\hline $\mathrm{C} 8$ & $1344(2)$ & 4064(4) & $1339(1)$ & $14(1)$ \\
\hline C9 & $1684(2)$ & $5321(4)$ & $2302(1)$ & $18(1)$ \\
\hline $\mathrm{C} 10$ & $1288(3)$ & $6607(4)$ & $2364(1)$ & $24(1)$ \\
\hline $\mathrm{C} 11$ & 1591(2) & $4456(4)$ & $2625(1)$ & $23(1)$ \\
\hline $\mathrm{C} 12$ & $2876(2)$ & 5463(4) & 1922(1) & $16(1)$ \\
\hline $\mathrm{C} 13$ & $2567(2)$ & $5530(4)$ & 2251(1) & $17(1)$ \\
\hline $\mathrm{C} 14$ & $1295(2)$ & $4700(4)$ & 1967(1) & $16(1)$ \\
\hline $\mathrm{C} 15$ & $1672(2)$ & $4656(4)$ & $1650(1)$ & $14(1)$ \\
\hline $\mathrm{C} 16$ & $5065(2)$ & $5177(4)$ & $1469(1)$ & $16(1)$ \\
\hline $\mathrm{C} 17$ & $5581(2)$ & $6188(4)$ & $1436(1)$ & $19(1)$ \\
\hline $\mathrm{C} 18$ & $6400(2)$ & 5997(4) & $1470(1)$ & $20(1)$ \\
\hline C19 & $6700(2)$ & $4808(4)$ & $1546(1)$ & $22(1)$ \\
\hline $\mathrm{C} 20$ & $6188(2)$ & $3789(4)$ & $1582(1)$ & $22(1)$ \\
\hline $\mathrm{C} 21$ & $5375(2)$ & $3966(4)$ & $1542(1)$ & $20(1)$ \\
\hline $\mathrm{C} 22$ & $3827(2)$ & 6811(4) & $1176(1)$ & $19(1)$ \\
\hline $\mathrm{C} 23$ & $3737(2)$ & $7968(4)$ & $1345(1)$ & $21(1)$ \\
\hline $\mathrm{C} 24$ & $3661(2)$ & 9093(4) & $1148(1)$ & $23(1)$ \\
\hline $\mathrm{C} 25$ & $3655(3)$ & $9036(4)$ & $782(1)$ & $28(1)$ \\
\hline
\end{tabular}




\begin{tabular}{|c|c|c|c|c|}
\hline $\mathrm{C} 26$ & $3737(3)$ & 7891(4) & $615(1)$ & $32(1)$ \\
\hline $\mathrm{C} 27$ & $3823(3)$ & 6783(4) & $809(1)$ & $27(1)$ \\
\hline $\mathrm{C} 28$ & $1782(2)$ & 5198(4) & $676(1)$ & $16(1)$ \\
\hline $\mathrm{C} 29$ & $1167(3)$ & 6053(4) & $713(1)$ & $23(1)$ \\
\hline $\mathrm{C} 30$ & $1027(3)$ & $7005(4)$ & $465(1)$ & $28(1)$ \\
\hline $\mathrm{C} 31$ & $1484(3)$ & $7106(4)$ & $176(1)$ & $28(1)$ \\
\hline $\mathrm{C} 32$ & 2095(3) & $6254(4)$ & $135(1)$ & $27(1)$ \\
\hline $\mathrm{C} 33$ & $2246(2)$ & $5319(4)$ & $385(1)$ & $23(1)$ \\
\hline $\mathrm{C} 34$ & $1542(2)$ & $2558(4)$ & $704(1)$ & $16(1)$ \\
\hline $\mathrm{C} 35$ & $950(2)$ & 2741(4) & $433(1)$ & $20(1)$ \\
\hline $\mathrm{C} 36$ & $697(2)$ & $1736(4)$ & $220(1)$ & $24(1)$ \\
\hline C 37 & $1020(2)$ & $548(4)$ & $273(1)$ & $25(1)$ \\
\hline C38 & $1602(3)$ & $339(4)$ & $542(1)$ & $28(1)$ \\
\hline C39 & $1859(2)$ & $1350(4)$ & $757(1)$ & $20(1)$ \\
\hline $\mathrm{Fe} 2$ & $8354(1)$ & $821(1)$ & $1174(1)$ & $14(1)$ \\
\hline $\mathrm{Cl} 3$ & 8999(1) & $1446(1)$ & $708(1)$ & $29(1)$ \\
\hline $\mathrm{Cl} 4$ & $8165(1)$ & $2042(1)$ & $1653(1)$ & $19(1)$ \\
\hline P3 & 6981(1) & $334(1)$ & $969(1)$ & $15(1)$ \\
\hline P4 & $9000(1)$ & $-1135(1)$ & $1400(1)$ & $14(1)$ \\
\hline $\mathrm{O} 2$ & $7423(1)$ & $-1032(2)$ & $1608(1)$ & $14(1)$ \\
\hline $\mathrm{C} 40$ & $6357(2)$ & $129(4)$ & $1345(1)$ & $15(1)$ \\
\hline $\mathrm{C} 41$ & $5596(2)$ & $642(4)$ & $1358(1)$ & $18(1)$ \\
\hline $\mathrm{C} 42$ & $5202(2)$ & $555(4)$ & $1669(1)$ & $22(1)$ \\
\hline $\mathrm{C} 43$ & $5562(2)$ & $7(4)$ & 1970(1) & $20(1)$ \\
\hline $\mathrm{C} 44$ & $8148(2)$ & $-1670(4)$ & $2525(1)$ & $24(1)$ \\
\hline $\mathrm{C} 45$ & $8949(2)$ & $-1833(4)$ & $2472(1)$ & $26(1)$ \\
\hline $\mathrm{C} 46$ & $9229(2)$ & $-1718(4)$ & $2135(1)$ & $20(1)$ \\
\hline $\mathrm{C} 47$ & $8706(2)$ & $-1438(4)$ & $1851(1)$ & $15(1)$ \\
\hline $\mathrm{C} 48$ & $6718(2)$ & $-1150(4)$ & $2301(1)$ & $16(1)$ \\
\hline C49 & $6316(2)$ & $-2437(4)$ & $2366(1)$ & $24(1)$ \\
\hline $\mathrm{C} 50$ & $6645(2)$ & $-285(4)$ & $2628(1)$ & $23(1)$ \\
\hline C51 & $6686(2)$ & $-473(4)$ & $1651(1)$ & $14(1)$ \\
\hline C52 & $6324(2)$ & $-531(4)$ & 1970(1) & $17(1)$ \\
\hline C53 & $7600(2)$ & $-1375(4)$ & $2244(1)$ & $16(1)$ \\
\hline C54 & $7902(2)$ & $-1281(4)$ & 1913(1) & $14(1)$ \\
\hline C55 & $6528(2)$ & 1651(4) & $717(1)$ & $19(1)$ \\
\hline
\end{tabular}




\begin{tabular}{|c|c|c|c|c|}
\hline C56 & $5913(2)$ & $1465(4)$ & $456(1)$ & $23(1)$ \\
\hline C57 & $5586(2)$ & $2508(5)$ & $272(1)$ & $28(1)$ \\
\hline $\mathrm{C} 58$ & $5865(3)$ & $3706(4)$ & $344(1)$ & $26(1)$ \\
\hline C59 & $6473(3)$ & $3905(4)$ & $601(1)$ & $27(1)$ \\
\hline C60 & $6803(2)$ & 2865(4) & $784(1)$ & $20(1)$ \\
\hline C61 & $6782(2)$ & $-1006(4)$ & $677(1)$ & $18(1)$ \\
\hline C62 & $6164(2)$ & $-1860(4)$ & $720(1)$ & $21(1)$ \\
\hline C63 & $6004(2)$ & $-2826(4)$ & $476(1)$ & $23(1)$ \\
\hline C64 & $6461(2)$ & $-2929(4)$ & $186(1)$ & $26(1)$ \\
\hline C65 & $7073(3)$ & $-2087(4)$ & $134(1)$ & $28(1)$ \\
\hline C66 & $7235(3)$ & $-1135(4)$ & $381(1)$ & $27(1)$ \\
\hline C67 & $8840(2)$ & $-2625(4)$ & $1158(1)$ & $14(1)$ \\
\hline C68 & $8724(2)$ & $-3786(4)$ & 1323(1) & $21(1)$ \\
\hline C69 & $8646(2)$ & $-4883(4)$ & $1124(1)$ & $26(1)$ \\
\hline $\mathrm{C} 70$ & $8681(2)$ & $-4842(4)$ & $761(1)$ & $26(1)$ \\
\hline C71 & 8791(3) & $-3701(5)$ & $592(1)$ & $34(1)$ \\
\hline C72 & $8863(3)$ & $-2583(4)$ & $789(1)$ & $26(1)$ \\
\hline $\mathrm{C} 73$ & $10076(2)$ & $-985(4)$ & $1456(1)$ & $18(1)$ \\
\hline C74 & $10598(2)$ & $-2012(4)$ & $1424(1)$ & $20(1)$ \\
\hline $\mathrm{C} 75$ & $11408(2)$ & $-1802(4)$ & $1462(1)$ & $23(1)$ \\
\hline C76 & $11704(2)$ & $-592(4)$ & 1531(1) & $21(1)$ \\
\hline C77 & $11202(2)$ & $415(4)$ & $1564(1)$ & $23(1)$ \\
\hline C78 & $10385(2)$ & $224(4)$ & $1518(1)$ & $18(1)$ \\
\hline
\end{tabular}


Table S21. Bond lengths $[\AA]$ and angles $\left[{ }^{\circ}\right]$ for neivf02.

\begin{tabular}{|c|c|c|c|}
\hline $\mathrm{Fe}(1)-\mathrm{Cl}(1)$ & $2.2137(12)$ & $\mathrm{C}(10)-\mathrm{H}(10 \mathrm{C})$ & 0.9800 \\
\hline $\mathrm{Fe}(1)-\mathrm{Cl}(2)$ & $2.2548(12)$ & $\mathrm{C}(11)-\mathrm{H}(11 \mathrm{~A})$ & 0.9800 \\
\hline $\mathrm{Fe}(1)-\mathrm{P}(1)$ & $2.4564(13)$ & $\mathrm{C}(11)-\mathrm{H}(11 \mathrm{~B})$ & 0.9800 \\
\hline $\mathrm{Fe}(1)-\mathrm{P}(2)$ & $2.4572(13)$ & $\mathrm{C}(11)-\mathrm{H}(11 \mathrm{C})$ & 0.9800 \\
\hline $\mathrm{P}(1)-\mathrm{C}(1)$ & $1.814(4)$ & $C(12)-C(13)$ & $1.377(5)$ \\
\hline $\mathrm{P}(1)-\mathrm{C}(22)$ & $1.823(4)$ & $C(14)-C(15)$ & $1.389(5)$ \\
\hline $\mathrm{P}(1)-\mathrm{C}(16)$ & $1.825(4)$ & $C(16)-C(17)$ & $1.385(5)$ \\
\hline $\mathrm{P}(2)-\mathrm{C}(28)$ & $1.811(4)$ & $C(16)-C(21)$ & $1.400(5)$ \\
\hline $\mathrm{P}(2)-\mathrm{C}(8)$ & $1.817(4)$ & $C(17)-C(18)$ & $1.396(5)$ \\
\hline $\mathrm{P}(2)-\mathrm{C}(34)$ & $1.827(4)$ & $\mathrm{C}(17)-\mathrm{H}(17)$ & 0.9500 \\
\hline $\mathrm{O}(1)-\mathrm{C}(15)$ & $1.381(4)$ & $C(18)-C(19)$ & $1.375(6)$ \\
\hline $\mathrm{O}(1)-\mathrm{C}(12)$ & $1.391(4)$ & $\mathrm{C}(18)-\mathrm{H}(18)$ & 0.9500 \\
\hline $\mathrm{C}(1)-\mathrm{C}(2)$ & $1.395(5)$ & $C(19)-C(20)$ & $1.390(6)$ \\
\hline$C(1)-C(12)$ & $1.406(5)$ & $\mathrm{C}(19)-\mathrm{H}(19)$ & 0.9500 \\
\hline $\mathrm{C}(2)-\mathrm{C}(3)$ & $1.384(5)$ & $C(20)-C(21)$ & $1.384(5)$ \\
\hline $\mathrm{C}(2)-\mathrm{H}(2)$ & 0.9500 & $\mathrm{C}(20)-\mathrm{H}(20)$ & 0.9500 \\
\hline$C(3)-C(4)$ & $1.386(6)$ & $\mathrm{C}(21)-\mathrm{H}(21)$ & 0.9500 \\
\hline $\mathrm{C}(3)-\mathrm{H}(3)$ & 0.9500 & $C(22)-C(27)$ & $1.386(6)$ \\
\hline$C(4)-C(13)$ & $1.404(5)$ & $C(22)-C(23)$ & $1.387(6)$ \\
\hline $\mathrm{C}(4)-\mathrm{H}(4)$ & 0.9500 & $C(23)-C(24)$ & $1.401(6)$ \\
\hline$C(5)-C(6)$ & $1.391(6)$ & $\mathrm{C}(23)-\mathrm{H}(23)$ & 0.9500 \\
\hline$C(5)-C(14)$ & $1.392(5)$ & $C(24)-C(25)$ & $1.384(6)$ \\
\hline $\mathrm{C}(5)-\mathrm{H}(5)$ & 0.9500 & $\mathrm{C}(24)-\mathrm{H}(24)$ & 0.9500 \\
\hline$C(6)-C(7)$ & $1.381(5)$ & $C(25)-C(26)$ & $1.372(6)$ \\
\hline $\mathrm{C}(6)-\mathrm{H}(6)$ & 0.9500 & $\mathrm{C}(25)-\mathrm{H}(25)$ & 0.9500 \\
\hline$C(7)-C(8)$ & $1.403(5)$ & $C(26)-C(27)$ & $1.382(6)$ \\
\hline $\mathrm{C}(7)-\mathrm{H}(7)$ & 0.9500 & $\mathrm{C}(26)-\mathrm{H}(26)$ & 0.9500 \\
\hline$C(8)-C(15)$ & $1.413(5)$ & $\mathrm{C}(27)-\mathrm{H}(27)$ & 0.9500 \\
\hline$C(9)-C(13)$ & $1.530(5)$ & $C(28)-C(29)$ & $1.388(5)$ \\
\hline$C(9)-C(10)$ & $1.535(5)$ & $C(28)-C(33)$ & $1.392(5)$ \\
\hline$C(9)-C(11)$ & $1.536(5)$ & $C(29)-C(30)$ & $1.382(6)$ \\
\hline$C(9)-C(14)$ & $1.536(5)$ & $\mathrm{C}(29)-\mathrm{H}(29)$ & 0.9500 \\
\hline $\mathrm{C}(10)-\mathrm{H}(10 \mathrm{~A})$ & 0.9800 & $C(30)-C(31)$ & $1.379(6)$ \\
\hline $\mathrm{C}(10)-\mathrm{H}(10 \mathrm{~B})$ & 0.9800 & $\mathrm{C}(30)-\mathrm{H}(30)$ & 0.9500 \\
\hline
\end{tabular}




\begin{tabular}{|c|c|c|c|}
\hline$C(31)-C(32)$ & $1.383(6)$ & $\mathrm{C}(44)-\mathrm{C}(45)$ & $1.391(6)$ \\
\hline $\mathrm{C}(31)-\mathrm{H}(31)$ & 0.9500 & $\mathrm{C}(44)-\mathrm{C}(53)$ & $1.401(5)$ \\
\hline$C(32)-C(33)$ & $1.378(6)$ & $\mathrm{C}(44)-\mathrm{H}(44)$ & 0.9500 \\
\hline $\mathrm{C}(32)-\mathrm{H}(32)$ & 0.9500 & $C(45)-C(46)$ & $1.392(5)$ \\
\hline $\mathrm{C}(33)-\mathrm{H}(33)$ & 0.9500 & $\mathrm{C}(45)-\mathrm{H}(45)$ & 0.9500 \\
\hline$C(34)-C(39)$ & $1.390(5)$ & $C(46)-C(47)$ & $1.379(5)$ \\
\hline$C(34)-C(35)$ & $1.401(5)$ & $\mathrm{C}(46)-\mathrm{H}(46)$ & 0.9500 \\
\hline$C(35)-C(36)$ & $1.383(6)$ & $\mathrm{C}(47)-\mathrm{C}(54)$ & $1.401(5)$ \\
\hline $\mathrm{C}(35)-\mathrm{H}(35)$ & 0.9500 & $\mathrm{C}(48)-\mathrm{C}(52)$ & $1.529(5)$ \\
\hline$C(36)-C(37)$ & $1.376(6)$ & $\mathrm{C}(48)-\mathrm{C}(53)$ & $1.536(5)$ \\
\hline $\mathrm{C}(36)-\mathrm{H}(36)$ & 0.9500 & $\mathrm{C}(48)-\mathrm{C}(49)$ & $1.542(5)$ \\
\hline$C(37)-C(38)$ & $1.388(6)$ & $\mathrm{C}(48)-\mathrm{C}(50)$ & $1.543(5)$ \\
\hline $\mathrm{C}(37)-\mathrm{H}(37)$ & 0.9500 & $\mathrm{C}(49)-\mathrm{H}(49 \mathrm{~A})$ & 0.9800 \\
\hline$C(38)-C(39)$ & $1.392(6)$ & $\mathrm{C}(49)-\mathrm{H}(49 \mathrm{~B})$ & 0.9800 \\
\hline $\mathrm{C}(38)-\mathrm{H}(38)$ & 0.9500 & $\mathrm{C}(49)-\mathrm{H}(49 \mathrm{C})$ & 0.9800 \\
\hline $\mathrm{C}(39)-\mathrm{H}(39)$ & 0.9500 & $\mathrm{C}(50)-\mathrm{H}(50 \mathrm{~A})$ & 0.9800 \\
\hline $\mathrm{Fe}(2)-\mathrm{Cl}(3)$ & $2.2247(12)$ & $\mathrm{C}(50)-\mathrm{H}(50 \mathrm{~B})$ & 0.9800 \\
\hline $\mathrm{Fe}(2)-\mathrm{Cl}(4)$ & $2.2565(12)$ & $\mathrm{C}(50)-\mathrm{H}(50 \mathrm{C})$ & 0.9800 \\
\hline $\mathrm{Fe}(2)-\mathrm{P}(3)$ & $2.4543(13)$ & $C(51)-C(52)$ & $1.383(5)$ \\
\hline $\mathrm{Fe}(2)-\mathrm{P}(4)$ & $2.4595(13)$ & $\mathrm{C}(53)-\mathrm{C}(54)$ & $1.382(5)$ \\
\hline $\mathrm{P}(3)-\mathrm{C}(61)$ & $1.811(4)$ & $C(55)-C(60)$ & $1.380(6)$ \\
\hline $\mathrm{P}(3)-\mathrm{C}(55)$ & $1.824(4)$ & $\mathrm{C}(55)-\mathrm{C}(56)$ & $1.401(5)$ \\
\hline $\mathrm{P}(3)-\mathrm{C}(40)$ & $1.831(4)$ & $C(56)-C(57)$ & $1.396(6)$ \\
\hline $\mathrm{P}(4)-\mathrm{C}(73)$ & $1.822(4)$ & $\mathrm{C}(56)-\mathrm{H}(56)$ & 0.9500 \\
\hline $\mathrm{P}(4)-\mathrm{C}(67)$ & $1.827(4)$ & $\mathrm{C}(57)-\mathrm{C}(58)$ & $1.368(6)$ \\
\hline $\mathrm{P}(4)-\mathrm{C}(47)$ & $1.831(4)$ & $\mathrm{C}(57)-\mathrm{H}(57)$ & 0.9500 \\
\hline $\mathrm{O}(2)-\mathrm{C}(54)$ & $1.390(4)$ & $\mathrm{C}(58)-\mathrm{C}(59)$ & $1.384(6)$ \\
\hline $\mathrm{O}(2)-\mathrm{C}(51)$ & $1.395(4)$ & $\mathrm{C}(58)-\mathrm{H}(58)$ & 0.9500 \\
\hline$C(40)-C(41)$ & $1.397(5)$ & $\mathrm{C}(59)-\mathrm{C}(60)$ & $1.395(6)$ \\
\hline$C(40)-C(51)$ & $1.401(5)$ & $\mathrm{C}(59)-\mathrm{H}(59)$ & 0.9500 \\
\hline$C(41)-C(42)$ & $1.388(5)$ & $\mathrm{C}(60)-\mathrm{H}(60)$ & 0.9500 \\
\hline $\mathrm{C}(41)-\mathrm{H}(41)$ & 0.9500 & $\mathrm{C}(61)-\mathrm{C}(62)$ & $1.395(5)$ \\
\hline$C(42)-C(43)$ & $1.380(6)$ & $\mathrm{C}(61)-\mathrm{C}(66)$ & $1.398(5)$ \\
\hline $\mathrm{C}(42)-\mathrm{H}(42)$ & 0.9500 & $\mathrm{C}(62)-\mathrm{C}(63)$ & $1.389(6)$ \\
\hline$C(43)-C(52)$ & $1.406(5)$ & $\mathrm{C}(62)-\mathrm{H}(62)$ & 0.9500 \\
\hline $\mathrm{C}(43)-\mathrm{H}(43)$ & 0.9500 & $\mathrm{C}(63)-\mathrm{C}(64)$ & $1.383(6)$ \\
\hline
\end{tabular}




\begin{tabular}{|c|c|c|c|}
\hline $\mathrm{C}(63)-\mathrm{H}(63)$ & 0.9500 & $\mathrm{C}(22)-\mathrm{P}(1)-\mathrm{C}(16)$ & $104.12(18)$ \\
\hline$C(64)-C(65)$ & $1.384(6)$ & $\mathrm{C}(1)-\mathrm{P}(1)-\mathrm{Fe}(1)$ & $108.13(13)$ \\
\hline $\mathrm{C}(64)-\mathrm{H}(64)$ & 0.9500 & $\mathrm{C}(22)-\mathrm{P}(1)-\mathrm{Fe}(1)$ & $120.12(14)$ \\
\hline$C(65)-C(66)$ & $1.385(6)$ & $\mathrm{C}(16)-\mathrm{P}(1)-\mathrm{Fe}(1)$ & $112.97(13)$ \\
\hline $\mathrm{C}(65)-\mathrm{H}(65)$ & 0.9500 & $\mathrm{C}(28)-\mathrm{P}(2)-\mathrm{C}(8)$ & $106.72(18)$ \\
\hline $\mathrm{C}(66)-\mathrm{H}(66)$ & 0.9500 & $\mathrm{C}(28)-\mathrm{P}(2)-\mathrm{C}(34)$ & $101.32(18)$ \\
\hline $\mathrm{C}(67)-\mathrm{C}(68)$ & $1.390(5)$ & $\mathrm{C}(8)-\mathrm{P}(2)-\mathrm{C}(34)$ & $106.02(18)$ \\
\hline$C(67)-C(72)$ & $1.397(5)$ & $\mathrm{C}(28)-\mathrm{P}(2)-\mathrm{Fe}(1)$ & $120.42(13)$ \\
\hline$C(68)-C(69)$ & $1.381(6)$ & $\mathrm{C}(8)-\mathrm{P}(2)-\mathrm{Fe}(1)$ & $109.82(13)$ \\
\hline $\mathrm{C}(68)-\mathrm{H}(68)$ & 0.9500 & $\mathrm{C}(34)-\mathrm{P}(2)-\mathrm{Fe}(1)$ & $111.42(13)$ \\
\hline $\mathrm{C}(69)-\mathrm{C}(70)$ & $1.374(6)$ & $\mathrm{C}(15)-\mathrm{O}(1)-\mathrm{C}(12)$ & $118.3(3)$ \\
\hline $\mathrm{C}(69)-\mathrm{H}(69)$ & 0.9500 & $C(2)-C(1)-C(12)$ & $117.2(4)$ \\
\hline $\mathrm{C}(70)-\mathrm{C}(71)$ & $1.378(6)$ & $\mathrm{C}(2)-\mathrm{C}(1)-\mathrm{P}(1)$ & $125.0(3)$ \\
\hline $\mathrm{C}(70)-\mathrm{H}(70)$ & 0.9500 & $\mathrm{C}(12)-\mathrm{C}(1)-\mathrm{P}(1)$ & $117.5(3)$ \\
\hline $\mathrm{C}(71)-\mathrm{C}(72)$ & $1.394(6)$ & $C(3)-C(2)-C(1)$ & $120.3(4)$ \\
\hline $\mathrm{C}(71)-\mathrm{H}(71)$ & 0.9500 & $\mathrm{C}(3)-\mathrm{C}(2)-\mathrm{H}(2)$ & 119.9 \\
\hline $\mathrm{C}(72)-\mathrm{H}(72)$ & 0.9500 & $\mathrm{C}(1)-\mathrm{C}(2)-\mathrm{H}(2)$ & 119.9 \\
\hline $\mathrm{C}(73)-\mathrm{C}(78)$ & $1.392(5)$ & $\mathrm{C}(2)-\mathrm{C}(3)-\mathrm{C}(4)$ & $120.4(4)$ \\
\hline$C(73)-C(74)$ & $1.405(5)$ & $\mathrm{C}(2)-\mathrm{C}(3)-\mathrm{H}(3)$ & 119.8 \\
\hline $\mathrm{C}(74)-\mathrm{C}(75)$ & $1.385(5)$ & $\mathrm{C}(4)-\mathrm{C}(3)-\mathrm{H}(3)$ & 119.8 \\
\hline $\mathrm{C}(74)-\mathrm{H}(74)$ & 0.9500 & $C(3)-C(4)-C(13)$ & $121.7(4)$ \\
\hline$C(75)-C(76)$ & $1.388(6)$ & $\mathrm{C}(3)-\mathrm{C}(4)-\mathrm{H}(4)$ & 119.2 \\
\hline $\mathrm{C}(75)-\mathrm{H}(75)$ & 0.9500 & $\mathrm{C}(13)-\mathrm{C}(4)-\mathrm{H}(4)$ & 119.2 \\
\hline $\mathrm{C}(76)-\mathrm{C}(77)$ & $1.369(5)$ & $C(6)-C(5)-C(14)$ & $122.1(4)$ \\
\hline $\mathrm{C}(76)-\mathrm{H}(76)$ & 0.9500 & $\mathrm{C}(6)-\mathrm{C}(5)-\mathrm{H}(5)$ & 118.9 \\
\hline $\mathrm{C}(77)-\mathrm{C}(78)$ & $1.393(5)$ & $\mathrm{C}(14)-\mathrm{C}(5)-\mathrm{H}(5)$ & 118.9 \\
\hline $\mathrm{C}(77)-\mathrm{H}(77)$ & 0.9500 & $C(7)-C(6)-C(5)$ & $120.0(4)$ \\
\hline $\mathrm{C}(78)-\mathrm{H}(78)$ & 0.9500 & $\mathrm{C}(7)-\mathrm{C}(6)-\mathrm{H}(6)$ & 120.0 \\
\hline $\mathrm{Cl}(1)-\mathrm{Fe}(1)-\mathrm{Cl}(2)$ & $122.12(5)$ & $\mathrm{C}(5)-\mathrm{C}(6)-\mathrm{H}(6)$ & 120.0 \\
\hline $\mathrm{Cl}(1)-\mathrm{Fe}(1)-\mathrm{P}(1)$ & $108.13(4)$ & $C(6)-C(7)-C(8)$ & $120.7(4)$ \\
\hline $\mathrm{Cl}(2)-\mathrm{Fe}(1)-\mathrm{P}(1)$ & $106.62(4)$ & $\mathrm{C}(6)-\mathrm{C}(7)-\mathrm{H}(7)$ & 119.6 \\
\hline $\mathrm{Cl}(1)-\mathrm{Fe}(1)-\mathrm{P}(2)$ & $107.50(5)$ & $\mathrm{C}(8)-\mathrm{C}(7)-\mathrm{H}(7)$ & 119.6 \\
\hline $\mathrm{Cl}(2)-\mathrm{Fe}(1)-\mathrm{P}(2)$ & $102.66(4)$ & $C(7)-C(8)-C(15)$ & $117.0(3)$ \\
\hline $\mathrm{P}(1)-\mathrm{Fe}(1)-\mathrm{P}(2)$ & $109.30(4)$ & $\mathrm{C}(7)-\mathrm{C}(8)-\mathrm{P}(2)$ & $124.6(3)$ \\
\hline $\mathrm{C}(1)-\mathrm{P}(1)-\mathrm{C}(22)$ & 105.96(19) & $\mathrm{C}(15)-\mathrm{C}(8)-\mathrm{P}(2)$ & $118.1(3)$ \\
\hline $\mathrm{C}(1)-\mathrm{P}(1)-\mathrm{C}(16)$ & $104.26(18)$ & C(13)-C(9)-C(10) & $109.2(3)$ \\
\hline
\end{tabular}




\begin{tabular}{|c|c|c|c|}
\hline $\mathrm{C}(13)-\mathrm{C}(9)-\mathrm{C}(11)$ & $109.4(3)$ & $\mathrm{C}(19)-\mathrm{C}(18)-\mathrm{H}(18)$ & 120.0 \\
\hline$C(10)-C(9)-C(11)$ & 109.7(3) & $\mathrm{C}(17)-\mathrm{C}(18)-\mathrm{H}(18)$ & 120.0 \\
\hline$C(13)-C(9)-C(14)$ & $109.2(3)$ & $C(18)-C(19)-C(20)$ & $120.0(4)$ \\
\hline$C(10)-C(9)-C(14)$ & $109.4(3)$ & $\mathrm{C}(18)-\mathrm{C}(19)-\mathrm{H}(19)$ & 120.0 \\
\hline$C(11)-C(9)-C(14)$ & $109.9(3)$ & $\mathrm{C}(20)-\mathrm{C}(19)-\mathrm{H}(19)$ & 120.0 \\
\hline $\mathrm{C}(9)-\mathrm{C}(10)-\mathrm{H}(10 \mathrm{~A})$ & 109.5 & $\mathrm{C}(21)-\mathrm{C}(20)-\mathrm{C}(19)$ & $120.2(4)$ \\
\hline $\mathrm{C}(9)-\mathrm{C}(10)-\mathrm{H}(10 \mathrm{~B})$ & 109.5 & $\mathrm{C}(21)-\mathrm{C}(20)-\mathrm{H}(20)$ & 119.9 \\
\hline $\mathrm{H}(10 \mathrm{~A})-\mathrm{C}(10)-\mathrm{H}(10 \mathrm{~B})$ & 109.5 & $\mathrm{C}(19)-\mathrm{C}(20)-\mathrm{H}(20)$ & 119.9 \\
\hline $\mathrm{C}(9)-\mathrm{C}(10)-\mathrm{H}(10 \mathrm{C})$ & 109.5 & $\mathrm{C}(20)-\mathrm{C}(21)-\mathrm{C}(16)$ & $120.1(4)$ \\
\hline $\mathrm{H}(10 \mathrm{~A})-\mathrm{C}(10)-\mathrm{H}(10 \mathrm{C})$ & 109.5 & $\mathrm{C}(20)-\mathrm{C}(21)-\mathrm{H}(21)$ & 120.0 \\
\hline $\mathrm{H}(10 \mathrm{~B})-\mathrm{C}(10)-\mathrm{H}(10 \mathrm{C})$ & 109.5 & $\mathrm{C}(16)-\mathrm{C}(21)-\mathrm{H}(21)$ & 120.0 \\
\hline $\mathrm{C}(9)-\mathrm{C}(11)-\mathrm{H}(11 \mathrm{~A})$ & 109.5 & $\mathrm{C}(27)-\mathrm{C}(22)-\mathrm{C}(23)$ & $118.9(4)$ \\
\hline $\mathrm{C}(9)-\mathrm{C}(11)-\mathrm{H}(11 \mathrm{~B})$ & 109.5 & $\mathrm{C}(27)-\mathrm{C}(22)-\mathrm{P}(1)$ & $118.0(3)$ \\
\hline $\mathrm{H}(11 \mathrm{~A})-\mathrm{C}(11)-\mathrm{H}(11 \mathrm{~B})$ & 109.5 & $\mathrm{C}(23)-\mathrm{C}(22)-\mathrm{P}(1)$ & $123.1(3)$ \\
\hline $\mathrm{C}(9)-\mathrm{C}(11)-\mathrm{H}(11 \mathrm{C})$ & 109.5 & $\mathrm{C}(22)-\mathrm{C}(23)-\mathrm{C}(24)$ & $120.6(4)$ \\
\hline $\mathrm{H}(11 \mathrm{~A})-\mathrm{C}(11)-\mathrm{H}(11 \mathrm{C})$ & 109.5 & $\mathrm{C}(22)-\mathrm{C}(23)-\mathrm{H}(23)$ & 119.7 \\
\hline $\mathrm{H}(11 \mathrm{~B})-\mathrm{C}(11)-\mathrm{H}(11 \mathrm{C})$ & 109.5 & $\mathrm{C}(24)-\mathrm{C}(23)-\mathrm{H}(23)$ & 119.7 \\
\hline $\mathrm{C}(13)-\mathrm{C}(12)-\mathrm{O}(1)$ & $122.2(3)$ & $\mathrm{C}(25)-\mathrm{C}(24)-\mathrm{C}(23)$ & $119.2(4)$ \\
\hline$C(13)-C(12)-C(1)$ & $124.4(4)$ & $\mathrm{C}(25)-\mathrm{C}(24)-\mathrm{H}(24)$ & 120.4 \\
\hline $\mathrm{O}(1)-\mathrm{C}(12)-\mathrm{C}(1)$ & $113.4(3)$ & $\mathrm{C}(23)-\mathrm{C}(24)-\mathrm{H}(24)$ & 120.4 \\
\hline$C(12)-C(13)-C(4)$ & $116.0(4)$ & $\mathrm{C}(26)-\mathrm{C}(25)-\mathrm{C}(24)$ & $120.2(4)$ \\
\hline$C(12)-C(13)-C(9)$ & $122.0(3)$ & $\mathrm{C}(26)-\mathrm{C}(25)-\mathrm{H}(25)$ & 119.9 \\
\hline$C(4)-C(13)-C(9)$ & $121.9(4)$ & $\mathrm{C}(24)-\mathrm{C}(25)-\mathrm{H}(25)$ & 119.9 \\
\hline$C(15)-C(14)-C(5)$ & $116.4(4)$ & $C(25)-C(26)-C(27)$ & $120.6(5)$ \\
\hline $\mathrm{C}(15)-\mathrm{C}(14)-\mathrm{C}(9)$ & $121.9(3)$ & $\mathrm{C}(25)-\mathrm{C}(26)-\mathrm{H}(26)$ & 119.7 \\
\hline$C(5)-C(14)-C(9)$ & $121.7(3)$ & $\mathrm{C}(27)-\mathrm{C}(26)-\mathrm{H}(26)$ & 119.7 \\
\hline $\mathrm{O}(1)-\mathrm{C}(15)-\mathrm{C}(14)$ & $121.9(3)$ & $\mathrm{C}(26)-\mathrm{C}(27)-\mathrm{C}(22)$ & $120.5(4)$ \\
\hline $\mathrm{O}(1)-\mathrm{C}(15)-\mathrm{C}(8)$ & $114.5(3)$ & $\mathrm{C}(26)-\mathrm{C}(27)-\mathrm{H}(27)$ & 119.8 \\
\hline $\mathrm{C}(14)-\mathrm{C}(15)-\mathrm{C}(8)$ & $123.7(3)$ & $\mathrm{C}(22)-\mathrm{C}(27)-\mathrm{H}(27)$ & 119.8 \\
\hline$C(17)-C(16)-C(21)$ & $119.3(4)$ & $\mathrm{C}(29)-\mathrm{C}(28)-\mathrm{C}(33)$ & $118.8(4)$ \\
\hline $\mathrm{C}(17)-\mathrm{C}(16)-\mathrm{P}(1)$ & $123.6(3)$ & $\mathrm{C}(29)-\mathrm{C}(28)-\mathrm{P}(2)$ & $123.3(3)$ \\
\hline $\mathrm{C}(21)-\mathrm{C}(16)-\mathrm{P}(1)$ & $117.2(3)$ & $\mathrm{C}(33)-\mathrm{C}(28)-\mathrm{P}(2)$ & $117.7(3)$ \\
\hline$C(16)-C(17)-C(18)$ & $120.3(4)$ & $\mathrm{C}(30)-\mathrm{C}(29)-\mathrm{C}(28)$ & $120.0(4)$ \\
\hline $\mathrm{C}(16)-\mathrm{C}(17)-\mathrm{H}(17)$ & 119.8 & $\mathrm{C}(30)-\mathrm{C}(29)-\mathrm{H}(29)$ & 120.0 \\
\hline $\mathrm{C}(18)-\mathrm{C}(17)-\mathrm{H}(17)$ & 119.8 & $\mathrm{C}(28)-\mathrm{C}(29)-\mathrm{H}(29)$ & 120.0 \\
\hline$C(19)-C(18)-C(17)$ & $120.1(4)$ & $\mathrm{C}(31)-\mathrm{C}(30)-\mathrm{C}(29)$ & $120.8(4)$ \\
\hline
\end{tabular}




\begin{tabular}{|c|c|c|c|}
\hline $\mathrm{C}(31)-\mathrm{C}(30)-\mathrm{H}(30)$ & 119.6 & $\mathrm{C}(61)-\mathrm{P}(3)-\mathrm{C}(40)$ & $106.63(18)$ \\
\hline $\mathrm{C}(29)-\mathrm{C}(30)-\mathrm{H}(30)$ & 119.6 & $C(55)-P(3)-C(40)$ & $104.58(18)$ \\
\hline $\mathrm{C}(30)-\mathrm{C}(31)-\mathrm{C}(32)$ & $119.7(4)$ & $\mathrm{C}(61)-\mathrm{P}(3)-\mathrm{Fe}(2)$ & $119.50(13)$ \\
\hline $\mathrm{C}(30)-\mathrm{C}(31)-\mathrm{H}(31)$ & 120.2 & $\mathrm{C}(55)-\mathrm{P}(3)-\mathrm{Fe}(2)$ & $111.40(14)$ \\
\hline $\mathrm{C}(32)-\mathrm{C}(31)-\mathrm{H}(31)$ & 120.2 & $\mathrm{C}(40)-\mathrm{P}(3)-\mathrm{Fe}(2)$ & $110.86(13)$ \\
\hline $\mathrm{C}(33)-\mathrm{C}(32)-\mathrm{C}(31)$ & 119.7(4) & $\mathrm{C}(73)-\mathrm{P}(4)-\mathrm{C}(67)$ & $104.47(18)$ \\
\hline C(33)-C(32)-H(32) & 120.1 & $\mathrm{C}(73)-\mathrm{P}(4)-\mathrm{C}(47)$ & $103.33(18)$ \\
\hline $\mathrm{C}(31)-\mathrm{C}(32)-\mathrm{H}(32)$ & 120.1 & $\mathrm{C}(67)-\mathrm{P}(4)-\mathrm{C}(47)$ & $105.86(18)$ \\
\hline $\mathrm{C}(32)-\mathrm{C}(33)-\mathrm{C}(28)$ & $121.0(4)$ & $\mathrm{C}(73)-\mathrm{P}(4)-\mathrm{Fe}(2)$ & $112.72(14)$ \\
\hline C(32)-C(33)-H(33) & 119.5 & $\mathrm{C}(67)-\mathrm{P}(4)-\mathrm{Fe}(2)$ & $119.85(13)$ \\
\hline $\mathrm{C}(28)-\mathrm{C}(33)-\mathrm{H}(33)$ & 119.5 & $\mathrm{C}(47)-\mathrm{P}(4)-\mathrm{Fe}(2)$ & $109.19(13)$ \\
\hline $\mathrm{C}(39)-\mathrm{C}(34)-\mathrm{C}(35)$ & $118.8(4)$ & $\mathrm{C}(54)-\mathrm{O}(2)-\mathrm{C}(51)$ & $117.7(3)$ \\
\hline $\mathrm{C}(39)-\mathrm{C}(34)-\mathrm{P}(2)$ & $118.2(3)$ & $\mathrm{C}(41)-\mathrm{C}(40)-\mathrm{C}(51)$ & $117.9(3)$ \\
\hline $\mathrm{C}(35)-\mathrm{C}(34)-\mathrm{P}(2)$ & $122.8(3)$ & $\mathrm{C}(41)-\mathrm{C}(40)-\mathrm{P}(3)$ & $123.6(3)$ \\
\hline $\mathrm{C}(36)-\mathrm{C}(35)-\mathrm{C}(34)$ & $120.2(4)$ & $\mathrm{C}(51)-\mathrm{C}(40)-\mathrm{P}(3)$ & $118.3(3)$ \\
\hline $\mathrm{C}(36)-\mathrm{C}(35)-\mathrm{H}(35)$ & 119.9 & $\mathrm{C}(42)-\mathrm{C}(41)-\mathrm{C}(40)$ & $119.4(4)$ \\
\hline $\mathrm{C}(34)-\mathrm{C}(35)-\mathrm{H}(35)$ & 119.9 & $\mathrm{C}(42)-\mathrm{C}(41)-\mathrm{H}(41)$ & 120.3 \\
\hline $\mathrm{C}(37)-\mathrm{C}(36)-\mathrm{C}(35)$ & $120.3(4)$ & $\mathrm{C}(40)-\mathrm{C}(41)-\mathrm{H}(41)$ & 120.3 \\
\hline $\mathrm{C}(37)-\mathrm{C}(36)-\mathrm{H}(36)$ & 119.8 & $C(43)-C(42)-C(41)$ & $120.9(4)$ \\
\hline $\mathrm{C}(35)-\mathrm{C}(36)-\mathrm{H}(36)$ & 119.8 & $\mathrm{C}(43)-\mathrm{C}(42)-\mathrm{H}(42)$ & 119.5 \\
\hline $\mathrm{C}(36)-\mathrm{C}(37)-\mathrm{C}(38)$ & $120.6(4)$ & $\mathrm{C}(41)-\mathrm{C}(42)-\mathrm{H}(42)$ & 119.5 \\
\hline $\mathrm{C}(36)-\mathrm{C}(37)-\mathrm{H}(37)$ & 119.7 & $C(42)-C(43)-C(52)$ & $121.8(4)$ \\
\hline C(38)-C(37)-H(37) & 119.7 & $\mathrm{C}(42)-\mathrm{C}(43)-\mathrm{H}(43)$ & 119.1 \\
\hline C(37)-C(38)-C(39) & $119.2(4)$ & $\mathrm{C}(52)-\mathrm{C}(43)-\mathrm{H}(43)$ & 119.1 \\
\hline $\mathrm{C}(37)-\mathrm{C}(38)-\mathrm{H}(38)$ & 120.4 & $C(45)-C(44)-C(53)$ & $121.5(4)$ \\
\hline $\mathrm{C}(39)-\mathrm{C}(38)-\mathrm{H}(38)$ & 120.4 & $\mathrm{C}(45)-\mathrm{C}(44)-\mathrm{H}(44)$ & 119.3 \\
\hline $\mathrm{C}(34)-\mathrm{C}(39)-\mathrm{C}(38)$ & $120.9(4)$ & $\mathrm{C}(53)-\mathrm{C}(44)-\mathrm{H}(44)$ & 119.3 \\
\hline $\mathrm{C}(34)-\mathrm{C}(39)-\mathrm{H}(39)$ & 119.6 & $C(44)-C(45)-C(46)$ & $120.5(4)$ \\
\hline C(38)-C(39)-H(39) & 119.6 & $\mathrm{C}(44)-\mathrm{C}(45)-\mathrm{H}(45)$ & 119.8 \\
\hline $\mathrm{Cl}(3)-\mathrm{Fe}(2)-\mathrm{Cl}(4)$ & $124.40(5)$ & $\mathrm{C}(46)-\mathrm{C}(45)-\mathrm{H}(45)$ & 119.8 \\
\hline $\mathrm{Cl}(3)-\mathrm{Fe}(2)-\mathrm{P}(3)$ & $108.03(5)$ & $C(47)-C(46)-C(45)$ & $119.6(4)$ \\
\hline $\mathrm{Cl}(4)-\mathrm{Fe}(2)-\mathrm{P}(3)$ & $101.29(4)$ & $\mathrm{C}(47)-\mathrm{C}(46)-\mathrm{H}(46)$ & 120.2 \\
\hline $\mathrm{Cl}(3)-\mathrm{Fe}(2)-\mathrm{P}(4)$ & $107.09(4)$ & $\mathrm{C}(45)-\mathrm{C}(46)-\mathrm{H}(46)$ & 120.2 \\
\hline $\mathrm{Cl}(4)-\mathrm{Fe}(2)-\mathrm{P}(4)$ & $106.34(4)$ & $C(46)-C(47)-C(54)$ & $118.6(4)$ \\
\hline $\mathrm{P}(3)-\mathrm{Fe}(2)-\mathrm{P}(4)$ & $109.00(4)$ & $\mathrm{C}(46)-\mathrm{C}(47)-\mathrm{P}(4)$ & $124.3(3)$ \\
\hline $\mathrm{C}(61)-\mathrm{P}(3)-\mathrm{C}(55)$ & $102.60(19)$ & $\mathrm{C}(54)-\mathrm{C}(47)-\mathrm{P}(4)$ & $117.0(3)$ \\
\hline
\end{tabular}




\begin{tabular}{|c|c|}
\hline$C(52)-C(48)-C(53)$ & $109.2(3)$ \\
\hline$C(52)-C(48)-C(49)$ & $109.4(3)$ \\
\hline$C(53)-C(48)-C(49)$ & $109.1(3)$ \\
\hline $\mathrm{C}(52)-\mathrm{C}(48)-\mathrm{C}(50)$ & $110.5(3)$ \\
\hline$C(53)-C(48)-C(50)$ & 109.1(3) \\
\hline $\mathrm{C}(49)-\mathrm{C}(48)-\mathrm{C}(50)$ & $109.6(3)$ \\
\hline $\mathrm{C}(48)-\mathrm{C}(49)-\mathrm{H}(49 \mathrm{~A})$ & 109.5 \\
\hline $\mathrm{C}(48)-\mathrm{C}(49)-\mathrm{H}(49 \mathrm{~B})$ & 109.5 \\
\hline $\mathrm{H}(49 \mathrm{~A})-\mathrm{C}(49)-\mathrm{H}(49 \mathrm{~B})$ & 109.5 \\
\hline $\mathrm{C}(48)-\mathrm{C}(49)-\mathrm{H}(49 \mathrm{C})$ & 109.5 \\
\hline $\mathrm{H}(49 \mathrm{~A})-\mathrm{C}(49)-\mathrm{H}(49 \mathrm{C})$ & 109.5 \\
\hline H(49B)-C(49)-H(49C) & 109.5 \\
\hline $\mathrm{C}(48)-\mathrm{C}(50)-\mathrm{H}(50 \mathrm{~A})$ & 109.5 \\
\hline $\mathrm{C}(48)-\mathrm{C}(50)-\mathrm{H}(50 \mathrm{~B})$ & 109.5 \\
\hline $\mathrm{H}(50 \mathrm{~A})-\mathrm{C}(50)-\mathrm{H}(50 \mathrm{~B})$ & 109.5 \\
\hline $\mathrm{C}(48)-\mathrm{C}(50)-\mathrm{H}(50 \mathrm{C})$ & 109.5 \\
\hline $\mathrm{H}(50 \mathrm{~A})-\mathrm{C}(50)-\mathrm{H}(50 \mathrm{C})$ & 109.5 \\
\hline $\mathrm{H}(50 \mathrm{~B})-\mathrm{C}(50)-\mathrm{H}(50 \mathrm{C})$ & 109.5 \\
\hline $\mathrm{C}(52)-\mathrm{C}(51)-\mathrm{O}(2)$ & $121.6(3)$ \\
\hline$C(52)-C(51)-C(40)$ & $124.2(3)$ \\
\hline $\mathrm{O}(2)-\mathrm{C}(51)-\mathrm{C}(40)$ & $114.2(3)$ \\
\hline $\mathrm{C}(51)-\mathrm{C}(52)-\mathrm{C}(43)$ & $115.7(4)$ \\
\hline $\mathrm{C}(51)-\mathrm{C}(52)-\mathrm{C}(48)$ & $122.6(3)$ \\
\hline$C(43)-C(52)-C(48)$ & $121.7(4)$ \\
\hline $\mathrm{C}(54)-\mathrm{C}(53)-\mathrm{C}(44)$ & $116.1(3)$ \\
\hline$C(54)-C(53)-C(48)$ & $122.0(3)$ \\
\hline $\mathrm{C}(44)-\mathrm{C}(53)-\mathrm{C}(48)$ & $121.9(3)$ \\
\hline $\mathrm{C}(53)-\mathrm{C}(54)-\mathrm{O}(2)$ & $122.3(3)$ \\
\hline$C(53)-C(54)-C(47)$ & $123.7(4)$ \\
\hline $\mathrm{O}(2)-\mathrm{C}(54)-\mathrm{C}(47)$ & $114.0(3)$ \\
\hline$C(60)-C(55)-C(56)$ & $119.1(4)$ \\
\hline $\mathrm{C}(60)-\mathrm{C}(55)-\mathrm{P}(3)$ & $118.8(3)$ \\
\hline $\mathrm{C}(56)-\mathrm{C}(55)-\mathrm{P}(3)$ & $122.1(3)$ \\
\hline $\mathrm{C}(57)-\mathrm{C}(56)-\mathrm{C}(55)$ & $119.6(4)$ \\
\hline $\mathrm{C}(57)-\mathrm{C}(56)-\mathrm{H}(56)$ & 120.2 \\
\hline $\mathrm{C}(55)-\mathrm{C}(56)-\mathrm{H}(56)$ & 120.2 \\
\hline
\end{tabular}

\begin{tabular}{|c|c|}
\hline $\mathrm{C}(58)-\mathrm{C}(57)-\mathrm{C}(56)$ & $120.4(4)$ \\
\hline $\mathrm{C}(58)-\mathrm{C}(57)-\mathrm{H}(57)$ & 119.8 \\
\hline $\mathrm{C}(56)-\mathrm{C}(57)-\mathrm{H}(57)$ & 119.8 \\
\hline$C(57)-C(58)-C(59)$ & $120.7(4)$ \\
\hline $\mathrm{C}(57)-\mathrm{C}(58)-\mathrm{H}(58)$ & 119.6 \\
\hline $\mathrm{C}(59)-\mathrm{C}(58)-\mathrm{H}(58)$ & 119.6 \\
\hline $\mathrm{C}(58)-\mathrm{C}(59)-\mathrm{C}(60)$ & 119.1(4) \\
\hline $\mathrm{C}(58)-\mathrm{C}(59)-\mathrm{H}(59)$ & 120.5 \\
\hline $\mathrm{C}(60)-\mathrm{C}(59)-\mathrm{H}(59)$ & 120.5 \\
\hline $\mathrm{C}(55)-\mathrm{C}(60)-\mathrm{C}(59)$ & 121.1(4) \\
\hline $\mathrm{C}(55)-\mathrm{C}(60)-\mathrm{H}(60)$ & 119.5 \\
\hline $\mathrm{C}(59)-\mathrm{C}(60)-\mathrm{H}(60)$ & 119.5 \\
\hline$C(62)-C(61)-C(66)$ & $118.7(4)$ \\
\hline $\mathrm{C}(62)-\mathrm{C}(61)-\mathrm{P}(3)$ & $123.0(3)$ \\
\hline $\mathrm{C}(66)-\mathrm{C}(61)-\mathrm{P}(3)$ & $118.2(3)$ \\
\hline $\mathrm{C}(63)-\mathrm{C}(62)-\mathrm{C}(61)$ & $120.8(4)$ \\
\hline $\mathrm{C}(63)-\mathrm{C}(62)-\mathrm{H}(62)$ & 119.6 \\
\hline $\mathrm{C}(61)-\mathrm{C}(62)-\mathrm{H}(62)$ & 119.6 \\
\hline $\mathrm{C}(64)-\mathrm{C}(63)-\mathrm{C}(62)$ & $119.3(4)$ \\
\hline $\mathrm{C}(64)-\mathrm{C}(63)-\mathrm{H}(63)$ & 120.4 \\
\hline $\mathrm{C}(62)-\mathrm{C}(63)-\mathrm{H}(63)$ & 120.4 \\
\hline$C(63)-C(64)-C(65)$ & $121.1(4)$ \\
\hline $\mathrm{C}(63)-\mathrm{C}(64)-\mathrm{H}(64)$ & 119.4 \\
\hline $\mathrm{C}(65)-\mathrm{C}(64)-\mathrm{H}(64)$ & 119.4 \\
\hline$C(64)-C(65)-C(66)$ & 119.3(4) \\
\hline $\mathrm{C}(64)-\mathrm{C}(65)-\mathrm{H}(65)$ & 120.3 \\
\hline $\mathrm{C}(66)-\mathrm{C}(65)-\mathrm{H}(65)$ & 120.3 \\
\hline $\mathrm{C}(65)-\mathrm{C}(66)-\mathrm{C}(61)$ & $120.8(4)$ \\
\hline $\mathrm{C}(65)-\mathrm{C}(66)-\mathrm{H}(66)$ & 119.6 \\
\hline $\mathrm{C}(61)-\mathrm{C}(66)-\mathrm{H}(66)$ & 119.6 \\
\hline $\mathrm{C}(68)-\mathrm{C}(67)-\mathrm{C}(72)$ & 119.2(4) \\
\hline $\mathrm{C}(68)-\mathrm{C}(67)-\mathrm{P}(4)$ & $123.6(3)$ \\
\hline $\mathrm{C}(72)-\mathrm{C}(67)-\mathrm{P}(4)$ & 117.2(3) \\
\hline $\mathrm{C}(69)-\mathrm{C}(68)-\mathrm{C}(67)$ & $120.2(4)$ \\
\hline $\mathrm{C}(69)-\mathrm{C}(68)-\mathrm{H}(68)$ & 119.9 \\
\hline $\mathrm{C}(67)-\mathrm{C}(68)-\mathrm{H}(68)$ & 119.9 \\
\hline
\end{tabular}




$\begin{array}{llll}\mathrm{C}(70)-\mathrm{C}(69)-\mathrm{C}(68) & 120.5(4) & \mathrm{C}(75)-\mathrm{C}(74)-\mathrm{C}(73) & 119.3(4) \\ \mathrm{C}(70)-\mathrm{C}(69)-\mathrm{H}(69) & 119.7 & \mathrm{C}(75)-\mathrm{C}(74)-\mathrm{H}(74) & 120.4 \\ \mathrm{C}(68)-\mathrm{C}(69)-\mathrm{H}(69) & 119.7 & \mathrm{C}(73)-\mathrm{C}(74)-\mathrm{H}(74) & 120.4 \\ \mathrm{C}(69)-\mathrm{C}(70)-\mathrm{C}(71) & 120.2(4) & \mathrm{C}(74)-\mathrm{C}(75)-\mathrm{C}(76) & 120.6(4) \\ \mathrm{C}(69)-\mathrm{C}(70)-\mathrm{H}(70) & 119.9 & \mathrm{C}(74)-\mathrm{C}(75)-\mathrm{H}(75) & 119.7 \\ \mathrm{C}(71)-\mathrm{C}(70)-\mathrm{H}(70) & 119.9 & \mathrm{C}(76)-\mathrm{C}(75)-\mathrm{H}(75) & 119.7 \\ \mathrm{C}(70)-\mathrm{C}(71)-\mathrm{C}(72) & 120.1(4) & \mathrm{C}(77)-\mathrm{C}(76)-\mathrm{C}(75) & 120.7(4) \\ \mathrm{C}(70)-\mathrm{C}(71)-\mathrm{H}(71) & 120.0 & \mathrm{C}(77)-\mathrm{C}(76)-\mathrm{H}(76) & 119.7 \\ \mathrm{C}(72)-\mathrm{C}(71)-\mathrm{H}(71) & 120.0 & \mathrm{C}(75)-\mathrm{C}(76)-\mathrm{H}(76) & 119.7 \\ \mathrm{C}(71)-\mathrm{C}(72)-\mathrm{C}(67) & 119.8(4) & \mathrm{C}(76)-\mathrm{C}(77)-\mathrm{C}(78) & 119.4(4) \\ \mathrm{C}(71)-\mathrm{C}(72)-\mathrm{H}(72) & 120.1 & \mathrm{C}(76)-\mathrm{C}(77)-\mathrm{H}(77) & 120.3 \\ \mathrm{C}(67)-\mathrm{C}(72)-\mathrm{H}(72) & 120.1 & \mathrm{C}(78)-\mathrm{C}(77)-\mathrm{H}(77) & 120.3 \\ \mathrm{C}(78)-\mathrm{C}(73)-\mathrm{C}(74) & 119.2(4) & \mathrm{C}(73)-\mathrm{C}(78)-\mathrm{C}(77) & 120.8(4) \\ \mathrm{C}(78)-\mathrm{C}(73)-\mathrm{P}(4) & 117.4(3) & \mathrm{C}(73)-\mathrm{C}(78)-\mathrm{H}(78) & 119.6 \\ \mathrm{C}(74)-\mathrm{C}(73)-\mathrm{P}(4) & 123.3(3) & \mathrm{C}(77)-\mathrm{C}(78)-\mathrm{H}(78) & 119.6\end{array}$


Table S22. Anisotropic displacement parameters $\left(\AA^{2} \times 10^{3}\right)$ for neivf02. The anisotropic displacement factor exponent takes the form: $-2 \pi^{2}\left[h^{2} a^{* 2} U_{11}+\ldots+2 h k a^{*} b^{*} U_{12}\right]$

\begin{tabular}{|c|c|c|c|c|c|c|}
\hline & $\mathrm{U}_{11}$ & $\mathrm{U}_{22}$ & $\mathrm{U}_{33}$ & $\mathrm{U}_{23}$ & $\mathrm{U}_{13}$ & $\mathrm{U}_{12}$ \\
\hline $\mathrm{Fe} 1$ & $11(1)$ & $16(1)$ & $17(1)$ & $0(1)$ & $1(1)$ & $0(1)$ \\
\hline $\mathrm{Cl1}$ & $22(1)$ & $35(1)$ & $26(1)$ & $-8(1)$ & $7(1)$ & $3(1)$ \\
\hline $\mathrm{Cl} 2$ & $18(1)$ & $20(1)$ & $21(1)$ & $5(1)$ & $2(1)$ & $1(1)$ \\
\hline $\mathrm{P} 1$ & $11(1)$ & $16(1)$ & $18(1)$ & $0(1)$ & $3(1)$ & $0(1)$ \\
\hline P2 & $12(1)$ & $13(1)$ & $17(1)$ & $0(1)$ & $1(1)$ & $1(1)$ \\
\hline $\mathrm{O} 1$ & $9(1)$ & $20(2)$ & $16(1)$ & $-1(1)$ & $0(1)$ & $-5(1)$ \\
\hline $\mathrm{C} 1$ & $15(2)$ & $17(2)$ & $15(2)$ & $-3(2)$ & $4(2)$ & $-2(2)$ \\
\hline $\mathrm{C} 2$ & $14(2)$ & $27(2)$ & $24(2)$ & $-2(2)$ & $3(2)$ & $-5(2)$ \\
\hline $\mathrm{C} 3$ & $24(2)$ & $34(3)$ & $19(2)$ & $-8(2)$ & $-1(2)$ & $-11(2)$ \\
\hline $\mathrm{C} 4$ & $27(2)$ & $27(2)$ & $19(2)$ & $-4(2)$ & $4(2)$ & $-3(2)$ \\
\hline C5 & $14(2)$ & $29(2)$ & $21(2)$ & $0(2)$ & $6(2)$ & $3(2)$ \\
\hline C6 & $9(2)$ & $25(2)$ & $29(2)$ & $-1(2)$ & $2(2)$ & $-5(2)$ \\
\hline C7 & $12(2)$ & $19(2)$ & $23(2)$ & $2(2)$ & $-3(2)$ & $1(2)$ \\
\hline $\mathrm{C} 8$ & $12(2)$ & $15(2)$ & $17(2)$ & $2(2)$ & $1(2)$ & $6(2)$ \\
\hline C9 & $18(2)$ & $20(2)$ & $17(2)$ & $-2(2)$ & $3(2)$ & $2(2)$ \\
\hline $\mathrm{C} 10$ & $26(2)$ & $23(2)$ & $22(2)$ & $-4(2)$ & $4(2)$ & $7(2)$ \\
\hline $\mathrm{C} 11$ & $18(2)$ & $30(3)$ & $22(2)$ & $-1(2)$ & $2(2)$ & $-2(2)$ \\
\hline $\mathrm{C} 12$ & $12(2)$ & $16(2)$ & $20(2)$ & $-1(2)$ & $-2(2)$ & $1(2)$ \\
\hline $\mathrm{C} 13$ & $15(2)$ & $15(2)$ & $20(2)$ & $-2(2)$ & $1(2)$ & $-3(2)$ \\
\hline $\mathrm{C} 14$ & $11(2)$ & $19(2)$ & $18(2)$ & $-1(2)$ & $3(2)$ & $4(2)$ \\
\hline $\mathrm{C} 15$ & $5(2)$ & $15(2)$ & $22(2)$ & $1(2)$ & $0(2)$ & $4(2)$ \\
\hline $\mathrm{C} 16$ & $12(2)$ & $19(2)$ & $17(2)$ & $-1(2)$ & $4(2)$ & $-1(2)$ \\
\hline $\mathrm{C} 17$ & $18(2)$ & $17(2)$ & $23(2)$ & $0(2)$ & $2(2)$ & $-2(2)$ \\
\hline $\mathrm{C} 18$ & $13(2)$ & $19(2)$ & $28(2)$ & $-1(2)$ & $3(2)$ & $-8(2)$ \\
\hline C19 & $12(2)$ & $34(3)$ & $21(2)$ & $-2(2)$ & $-2(2)$ & $6(2)$ \\
\hline $\mathrm{C} 20$ & $17(2)$ & $25(2)$ & $23(2)$ & $1(2)$ & $0(2)$ & $4(2)$ \\
\hline $\mathrm{C} 21$ & $19(2)$ & $23(2)$ & $20(2)$ & $1(2)$ & $2(2)$ & $-1(2)$ \\
\hline $\mathrm{C} 22$ & $6(2)$ & $29(2)$ & $23(2)$ & $-3(2)$ & $-1(2)$ & $-1(2)$ \\
\hline $\mathrm{C} 23$ & $17(2)$ & $25(2)$ & $21(2)$ & $-3(2)$ & $0(2)$ & $-1(2)$ \\
\hline $\mathrm{C} 24$ & $22(2)$ & $11(2)$ & $37(3)$ & $-2(2)$ & $5(2)$ & $2(2)$ \\
\hline $\mathrm{C} 25$ & $24(2)$ & $18(2)$ & $41(3)$ & $8(2)$ & $1(2)$ & $1(2)$ \\
\hline
\end{tabular}




\begin{tabular}{|c|c|c|c|c|c|c|}
\hline $\mathrm{C} 26$ & $41(3)$ & $29(3)$ & $27(3)$ & $4(2)$ & $4(2)$ & $2(2)$ \\
\hline $\mathrm{C} 27$ & $32(3)$ & $23(2)$ & 27(3) & $-3(2)$ & $3(2)$ & $4(2)$ \\
\hline $\mathrm{C} 28$ & $14(2)$ & $13(2)$ & $20(2)$ & $-2(2)$ & $-4(2)$ & $-1(2)$ \\
\hline C29 & $27(2)$ & $20(2)$ & $22(2)$ & $1(2)$ & $3(2)$ & $3(2)$ \\
\hline C30 & $33(3)$ & $22(2)$ & 29(3) & $2(2)$ & $1(2)$ & $7(2)$ \\
\hline C31 & $42(3)$ & $20(2)$ & $21(2)$ & $4(2)$ & $-4(2)$ & $0(2)$ \\
\hline C32 & $29(2)$ & $27(3)$ & $25(2)$ & $2(2)$ & $6(2)$ & $0(2)$ \\
\hline $\mathrm{C} 33$ & $20(2)$ & $22(2)$ & $27(2)$ & $3(2)$ & $3(2)$ & $6(2)$ \\
\hline C34 & $17(2)$ & $13(2)$ & $17(2)$ & $0(2)$ & $4(2)$ & $-2(2)$ \\
\hline C35 & $19(2)$ & $16(2)$ & $24(2)$ & $1(2)$ & $2(2)$ & $1(2)$ \\
\hline C36 & $18(2)$ & $27(2)$ & $27(2)$ & $-2(2)$ & $-7(2)$ & $-1(2)$ \\
\hline C37 & $22(2)$ & $20(2)$ & $31(3)$ & $-8(2)$ & $-2(2)$ & $-3(2)$ \\
\hline C38 & $27(2)$ & $19(2)$ & $38(3)$ & $-5(2)$ & $-4(2)$ & $3(2)$ \\
\hline C39 & $18(2)$ & $18(2)$ & $24(2)$ & $0(2)$ & $-3(2)$ & $-1(2)$ \\
\hline $\mathrm{Fe} 2$ & $10(1)$ & $15(1)$ & $17(1)$ & $1(1)$ & $2(1)$ & $1(1)$ \\
\hline $\mathrm{Cl} 3$ & $23(1)$ & $39(1)$ & $26(1)$ & 11(1) & $8(1)$ & $-2(1)$ \\
\hline $\mathrm{Cl} 4$ & $17(1)$ & $18(1)$ & 21(1) & $-4(1)$ & $1(1)$ & $1(1)$ \\
\hline P3 & $10(1)$ & $19(1)$ & $16(1)$ & $1(1)$ & $1(1)$ & $1(1)$ \\
\hline P4 & $10(1)$ & $15(1)$ & $18(1)$ & $0(1)$ & $2(1)$ & $1(1)$ \\
\hline $\mathrm{O} 2$ & $12(1)$ & $18(2)$ & $14(1)$ & $-1(1)$ & $2(1)$ & $2(1)$ \\
\hline C40 & $11(2)$ & $16(2)$ & $19(2)$ & $-2(2)$ & $4(2)$ & $1(2)$ \\
\hline C41 & $15(2)$ & $18(2)$ & $21(2)$ & $3(2)$ & $-2(2)$ & $4(2)$ \\
\hline $\mathrm{C} 42$ & $10(2)$ & $28(2)$ & $27(2)$ & $0(2)$ & $4(2)$ & $2(2)$ \\
\hline $\mathrm{C} 43$ & $15(2)$ & $26(2)$ & $20(2)$ & $-3(2)$ & $6(2)$ & $-2(2)$ \\
\hline C44 & $22(2)$ & $34(3)$ & $17(2)$ & $3(2)$ & $5(2)$ & $9(2)$ \\
\hline $\mathrm{C} 45$ & $19(2)$ & $40(3)$ & $20(2)$ & $3(2)$ & $-2(2)$ & $8(2)$ \\
\hline C46 & $11(2)$ & $24(2)$ & $23(2)$ & $4(2)$ & $2(2)$ & $7(2)$ \\
\hline C47 & $15(2)$ & $14(2)$ & $17(2)$ & $2(2)$ & $3(2)$ & $-1(2)$ \\
\hline C48 & $11(2)$ & $22(2)$ & $15(2)$ & $-1(2)$ & $3(2)$ & $-2(2)$ \\
\hline C49 & $23(2)$ & $30(3)$ & $18(2)$ & $6(2)$ & $2(2)$ & $-4(2)$ \\
\hline C50 & $13(2)$ & $30(3)$ & $26(2)$ & $-3(2)$ & $1(2)$ & $0(2)$ \\
\hline C51 & $6(2)$ & $16(2)$ & $20(2)$ & $-3(2)$ & $1(2)$ & $1(2)$ \\
\hline C52 & $13(2)$ & $15(2)$ & $23(2)$ & $1(2)$ & $1(2)$ & $-3(2)$ \\
\hline C53 & $12(2)$ & $17(2)$ & $18(2)$ & $1(2)$ & $1(2)$ & $-1(2)$ \\
\hline C54 & $13(2)$ & $15(2)$ & $15(2)$ & $1(2)$ & $-1(2)$ & $0(2)$ \\
\hline C55 & $11(2)$ & $33(3)$ & $14(2)$ & $3(2)$ & $2(2)$ & $5(2)$ \\
\hline
\end{tabular}




\begin{tabular}{lllllll} 
C56 & $19(2)$ & $30(3)$ & $20(2)$ & $4(2)$ & $-1(2)$ & $0(2)$ \\
C57 & $18(2)$ & $42(3)$ & $22(2)$ & $4(2)$ & $-3(2)$ & $6(2)$ \\
C58 & $23(2)$ & $26(3)$ & $30(3)$ & $7(2)$ & $3(2)$ & $12(2)$ \\
C59 & $30(2)$ & $19(2)$ & $32(3)$ & $3(2)$ & $1(2)$ & $5(2)$ \\
C60 & $16(2)$ & $23(2)$ & $22(2)$ & $-2(2)$ & $0(2)$ & $0(2)$ \\
C61 & $16(2)$ & $21(2)$ & $18(2)$ & $1(2)$ & $-1(2)$ & $1(2)$ \\
C62 & $17(2)$ & $22(2)$ & $24(2)$ & $3(2)$ & $1(2)$ & $0(2)$ \\
C63 & $24(2)$ & $21(2)$ & $24(2)$ & $-2(2)$ & $-3(2)$ & $-6(2)$ \\
C64 & $23(2)$ & $28(3)$ & $25(2)$ & $-6(2)$ & $-2(2)$ & $1(2)$ \\
C65 & $25(2)$ & $35(3)$ & $24(2)$ & $-8(2)$ & $5(2)$ & $-2(2)$ \\
C66 & $24(2)$ & $34(3)$ & $24(2)$ & $-8(2)$ & $5(2)$ & $-5(2)$ \\
C67 & $10(2)$ & $13(2)$ & $19(2)$ & $-3(2)$ & $1(2)$ & $2(2)$ \\
C68 & $21(2)$ & $17(2)$ & $24(2)$ & $2(2)$ & $3(2)$ & $-3(2)$ \\
C69 & $18(2)$ & $23(2)$ & $37(3)$ & $6(2)$ & $3(2)$ & $-1(2)$ \\
C70 & $27(2)$ & $26(3)$ & $25(2)$ & $-9(2)$ & $-3(2)$ & $-2(2)$ \\
C71 & $46(3)$ & $31(3)$ & $24(3)$ & $-6(2)$ & $6(2)$ & $2(2)$ \\
C72 & $38(3)$ & $15(2)$ & $25(2)$ & $-1(2)$ & $4(2)$ & $2(2)$ \\
C73 & $13(2)$ & $23(2)$ & $17(2)$ & $2(2)$ & $1(2)$ & $-3(2)$ \\
C74 & $14(2)$ & $20(2)$ & $27(2)$ & $-2(2)$ & $4(2)$ & $-1(2)$ \\
C75 & $15(2)$ & $28(3)$ & $28(3)$ & $-3(2)$ & $4(2)$ & $3(2)$ \\
C76 & $10(2)$ & $29(2)$ & $23(2)$ & $1(2)$ & $1(2)$ & $4(2)$ \\
C77 & $19(2)$ & $20(2)$ & $28(2)$ & $2(2)$ & $-3(2)$ & $-3(2)$ \\
C78 & $17(2)$ & $13(2)$ & $24(2)$ & $2(2)$ & $2(2)$ & $2(2)$ \\
& & & & & & \\
\hline
\end{tabular}


Table S23. Hydrogen coordinates $\left(\times 10^{4}\right)$ and isotropic displacement parameters $\left(\AA^{2} \times 10^{3}\right)$ for neivf02.

\begin{tabular}{|c|c|c|c|c|}
\hline & $\mathrm{x}$ & $\mathrm{y}$ & $\mathrm{z}$ & $\mathrm{U}(\mathrm{eq})$ \\
\hline $\mathrm{H} 2$ & 4752 & 5961 & 2127 & 26 \\
\hline H3 & 4263 & 6178 & 2688 & 31 \\
\hline $\mathrm{H} 4$ & 2920 & 5906 & 2767 & 29 \\
\hline H5 & 251 & 4221 & 2172 & 25 \\
\hline H6 & -348 & 3300 & 1664 & 25 \\
\hline $\mathrm{H} 7$ & 328 & 3181 & 1146 & 22 \\
\hline H10A & 1335 & 7148 & 2155 & 36 \\
\hline H10B & 1550 & 7020 & 2572 & 36 \\
\hline $\mathrm{H} 10 \mathrm{C}$ & 726 & 6475 & 2405 & 36 \\
\hline H11A & 1878 & 3660 & 2592 & 35 \\
\hline H11B & 1028 & 4274 & 2648 & 35 \\
\hline $\mathrm{H} 11 \mathrm{C}$ & 1809 & 4883 & 2840 & 35 \\
\hline H17 & 5375 & 7015 & 1389 & 23 \\
\hline H18 & 6751 & 6688 & 1439 & 24 \\
\hline H19 & 7258 & 4684 & 1576 & 27 \\
\hline $\mathrm{H} 20$ & 6397 & 2967 & 1633 & 26 \\
\hline H21 & 5027 & 3266 & 1565 & 24 \\
\hline $\mathrm{H} 23$ & 3726 & 7997 & 1596 & 25 \\
\hline $\mathrm{H} 24$ & 3614 & 9886 & 1265 & 28 \\
\hline $\mathrm{H} 25$ & 3595 & 9792 & 645 & 33 \\
\hline $\mathrm{H} 26$ & 3733 & 7859 & 363 & 39 \\
\hline $\mathrm{H} 27$ & 3881 & 5996 & 690 & 32 \\
\hline H29 & 842 & 5984 & 910 & 27 \\
\hline H30 & 612 & 7597 & 495 & 34 \\
\hline H31 & 1379 & 7757 & 5 & 34 \\
\hline H32 & 2409 & 6314 & -65 & 32 \\
\hline H33 & 2674 & 4747 & 358 & 28 \\
\hline H35 & 722 & 3558 & 396 & 24 \\
\hline H36 & 297 & 1867 & 35 & 29 \\
\hline H37 & 843 & -136 & 124 & 30 \\
\hline
\end{tabular}




\begin{tabular}{|c|c|c|c|c|}
\hline H38 & 1821 & -484 & 579 & 34 \\
\hline H39 & 2256 & 1212 & 942 & 24 \\
\hline H41 & 5350 & 1047 & 1155 & 22 \\
\hline $\mathrm{H} 42$ & 4678 & 878 & 1676 & 26 \\
\hline H43 & 5287 & -8 & 2182 & 24 \\
\hline H44 & 7967 & -1761 & 2758 & 29 \\
\hline $\mathrm{H} 45$ & 9309 & -2024 & 2669 & 32 \\
\hline $\mathrm{H} 46$ & 9777 & -1832 & 2100 & 23 \\
\hline H49A & 6360 & -2984 & 2158 & 36 \\
\hline H49B & 5754 & -2299 & 2407 & 36 \\
\hline $\mathrm{H} 49 \mathrm{C}$ & 6577 & -2846 & 2575 & 36 \\
\hline $\mathrm{H} 50 \mathrm{~A}$ & 6887 & -706 & 2839 & 34 \\
\hline H50B & 6084 & -118 & 2661 & 34 \\
\hline $\mathrm{H} 50 \mathrm{C}$ & 6920 & 519 & 2590 & 34 \\
\hline H56 & 5720 & 634 & 404 & 28 \\
\hline H57 & 5168 & 2385 & 96 & 33 \\
\hline H58 & 5638 & 4408 & 217 & 32 \\
\hline H59 & 6663 & 4739 & 651 & 32 \\
\hline H60 & 7224 & 2995 & 959 & 24 \\
\hline H62 & 5849 & -1781 & 920 & 25 \\
\hline H63 & 5585 & -3408 & 508 & 28 \\
\hline H64 & 6353 & -3589 & 18 & 31 \\
\hline H65 & 7379 & -2162 & -68 & 34 \\
\hline H66 & 7660 & -563 & 349 & 33 \\
\hline H68 & 8698 & -3824 & 1573 & 25 \\
\hline H69 & 8567 & -5672 & 1238 & 31 \\
\hline H70 & 8629 & -5603 & 626 & 31 \\
\hline H71 & 8817 & -3677 & 342 & 40 \\
\hline H72 & 8929 & -1794 & 673 & 31 \\
\hline H74 & 10397 & -2842 & 1378 & 24 \\
\hline H75 & 11764 & -2491 & 1440 & 28 \\
\hline H76 & 12262 & -462 & 1556 & 25 \\
\hline H77 & 11407 & 1237 & 1618 & 27 \\
\hline H78 & 10036 & 928 & 1529 & 22 \\
\hline
\end{tabular}


Table S24. Torsion angles $\left[{ }^{\circ}\right]$ for neivf02.

\begin{tabular}{|c|c|c|c|}
\hline C22-P1-C1-C2 & $-100.1(4)$ & C14-C9-C13-C12 & $15.2(5)$ \\
\hline C16-P1-C1-C2 & $9.5(4)$ & $\mathrm{C} 10-\mathrm{C} 9-\mathrm{C} 13-\mathrm{C} 4$ & $74.6(5)$ \\
\hline Fe1-P1-C1-C2 & $129.9(3)$ & C11-C9-C13-C4 & $-45.5(5)$ \\
\hline C22-P1-C1-C12 & $85.4(3)$ & C14-C9-C13-C4 & $-165.8(4)$ \\
\hline C16-P1-C1-C12 & $-165.1(3)$ & C6-C5-C14-C15 & $1.6(6)$ \\
\hline Fe1-P1-C1-C12 & $-44.6(3)$ & $\mathrm{C} 6-\mathrm{C} 5-\mathrm{C} 14-\mathrm{C} 9$ & $179.5(4)$ \\
\hline $\mathrm{C} 12-\mathrm{C} 1-\mathrm{C} 2-\mathrm{C} 3$ & $-0.9(6)$ & C13-C9-C14-C15 & $-14.2(5)$ \\
\hline P1-C1-C2-C3 & $-175.4(3)$ & C10-C9-C14-C15 & $105.3(4)$ \\
\hline $\mathrm{C} 1-\mathrm{C} 2-\mathrm{C} 3-\mathrm{C} 4$ & $1.4(7)$ & C11-C9-C14-C15 & $-134.2(4)$ \\
\hline $\mathrm{C} 2-\mathrm{C} 3-\mathrm{C} 4-\mathrm{C} 13$ & $0.1(7)$ & C13-C9-C14-C5 & 168.1(4) \\
\hline $\mathrm{C} 14-\mathrm{C} 5-\mathrm{C} 6-\mathrm{C} 7$ & $0.5(6)$ & $\mathrm{C} 10-\mathrm{C} 9-\mathrm{C} 14-\mathrm{C} 5$ & $-72.4(5)$ \\
\hline $\mathrm{C} 5-\mathrm{C} 6-\mathrm{C} 7-\mathrm{C} 8$ & $-0.5(6)$ & $\mathrm{C} 11-\mathrm{C} 9-\mathrm{C} 14-\mathrm{C} 5$ & $48.1(5)$ \\
\hline $\mathrm{C} 6-\mathrm{C} 7-\mathrm{C} 8-\mathrm{C} 15$ & $-1.6(6)$ & C12-O1-C15-C14 & $19.0(5)$ \\
\hline C6-C7-C8-P2 & $172.1(3)$ & $\mathrm{C} 12-\mathrm{O} 1-\mathrm{C} 15-\mathrm{C} 8$ & $-161.2(3)$ \\
\hline $\mathrm{C} 28-\mathrm{P} 2-\mathrm{C} 8-\mathrm{C} 7$ & $91.0(4)$ & $\mathrm{C} 5-\mathrm{C} 14-\mathrm{C} 15-\mathrm{O} 1$ & $175.9(3)$ \\
\hline C34-P2-C8-C7 & $-16.4(4)$ & C9-C14-C15-O1 & $-2.0(6)$ \\
\hline Fe1-P2-C8-C7 & $-136.9(3)$ & $\mathrm{C} 5-\mathrm{C} 14-\mathrm{C} 15-\mathrm{C} 8$ & $-3.9(6)$ \\
\hline C28-P2-C8-C15 & $-95.4(3)$ & C9-C14-C15-C8 & $178.2(4)$ \\
\hline C34-P2-C8-C15 & $157.2(3)$ & $\mathrm{C} 7-\mathrm{C} 8-\mathrm{C} 15-\mathrm{O} 1$ & $-175.9(3)$ \\
\hline Fe1-P2-C8-C15 & $36.7(3)$ & $\mathrm{P} 2-\mathrm{C} 8-\mathrm{C} 15-\mathrm{O} 1$ & $10.0(4)$ \\
\hline $\mathrm{C} 15-\mathrm{O} 1-\mathrm{C} 12-\mathrm{C} 13$ & $-18.1(5)$ & C7-C8-C15-C14 & $3.9(6)$ \\
\hline $\mathrm{C} 15-\mathrm{O} 1-\mathrm{C} 12-\mathrm{C} 1$ & 160.1(3) & $\mathrm{P} 2-\mathrm{C} 8-\mathrm{C} 15-\mathrm{C} 14$ & $-170.2(3)$ \\
\hline $\mathrm{C} 2-\mathrm{C} 1-\mathrm{C} 12-\mathrm{C} 13$ & $-1.4(6)$ & C1-P1-C16-C17 & $-92.3(4)$ \\
\hline $\mathrm{P} 1-\mathrm{C} 1-\mathrm{C} 12-\mathrm{C} 13$ & $173.6(3)$ & C22-P1-C16-C17 & $18.6(4)$ \\
\hline $\mathrm{C} 2-\mathrm{C} 1-\mathrm{C} 12-\mathrm{O} 1$ & $-179.5(3)$ & Fe1-P1-C16-C17 & $150.5(3)$ \\
\hline $\mathrm{P} 1-\mathrm{C} 1-\mathrm{C} 12-\mathrm{O} 1$ & $-4.5(5)$ & $\mathrm{C} 1-\mathrm{P} 1-\mathrm{C} 16-\mathrm{C} 21$ & $88.6(3)$ \\
\hline $\mathrm{O} 1-\mathrm{C} 12-\mathrm{C} 13-\mathrm{C} 4$ & $-179.2(4)$ & C22-P1-C16-C21 & $-160.5(3)$ \\
\hline $\mathrm{C} 1-\mathrm{C} 12-\mathrm{C} 13-\mathrm{C} 4$ & $2.9(6)$ & Fe1-P1-C16-C21 & $-28.6(3)$ \\
\hline $\mathrm{O} 1-\mathrm{C} 12-\mathrm{C} 13-\mathrm{C} 9$ & $-0.1(6)$ & C21-C16-C17-C18 & $1.0(6)$ \\
\hline C1-C12-C13-C9 & $-178.1(4)$ & P1-C16-C17-C18 & $-178.1(3)$ \\
\hline C3-C4-C13-C12 & $-2.2(6)$ & C16-C17-C18-C19 & $-1.9(6)$ \\
\hline C3-C4-C13-C9 & 178.7(4) & C17-C18-C19-C20 & $1.6(6)$ \\
\hline C10-C9-C13-C12 & $-104.5(4)$ & C18-C19-C20-C21 & $-0.4(6)$ \\
\hline C11-C9-C13-C12 & $135.5(4)$ & C19-C20-C21-C16 & $-0.5(6)$ \\
\hline
\end{tabular}




\begin{tabular}{|c|c|c|c|}
\hline C17-C16-C21-C20 & $0.2(6)$ & C39-C34-C35-C36 & $-0.9(6)$ \\
\hline P1-C16-C21-C20 & 179.3(3) & P2-C34-C35-C36 & 174.1(3) \\
\hline $\mathrm{C} 1-\mathrm{P} 1-\mathrm{C} 22-\mathrm{C} 27$ & $-164.5(3)$ & C34-C35-C36-C37 & $0.4(6)$ \\
\hline C16-P1-C22-C27 & $85.9(3)$ & C35-C36-C37-C38 & $0.2(7)$ \\
\hline $\mathrm{Fe} 1-\mathrm{P} 1-\mathrm{C} 22-\mathrm{C} 27$ & $-41.8(4)$ & C36-C37-C38-C39 & $-0.4(7)$ \\
\hline C1-P1-C22-C23 & $17.8(4)$ & C35-C34-C39-C38 & $0.8(6)$ \\
\hline C16-P1-C22-C23 & $-91.8(3)$ & P2-C34-C39-C38 & $-174.5(3)$ \\
\hline $\mathrm{Fe} 1-\mathrm{P} 1-\mathrm{C} 22-\mathrm{C} 23$ & $140.5(3)$ & C37-C38-C39-C34 & $-0.1(7)$ \\
\hline $\mathrm{C} 27-\mathrm{C} 22-\mathrm{C} 23-\mathrm{C} 24$ & $-1.4(6)$ & C61-P3-C40-C41 & $-91.9(4)$ \\
\hline P1-C22-C23-C24 & $176.3(3)$ & C55-P3-C40-C41 & $16.3(4)$ \\
\hline $\mathrm{C} 22-\mathrm{C} 23-\mathrm{C} 24-\mathrm{C} 25$ & $1.8(6)$ & Fe2-P3-C40-C41 & $136.5(3)$ \\
\hline $\mathrm{C} 23-\mathrm{C} 24-\mathrm{C} 25-\mathrm{C} 26$ & $-1.1(6)$ & C61-P3-C40-C51 & $94.2(3)$ \\
\hline C24-C25-C26-C27 & $0.1(7)$ & C55-P3-C40-C51 & $-157.5(3)$ \\
\hline $\mathrm{C} 25-\mathrm{C} 26-\mathrm{C} 27-\mathrm{C} 22$ & $0.2(7)$ & Fe2-P3-C40-C51 & $-37.3(3)$ \\
\hline $\mathrm{C} 23-\mathrm{C} 22-\mathrm{C} 27-\mathrm{C} 26$ & $0.4(6)$ & $\mathrm{C} 51-\mathrm{C} 40-\mathrm{C} 41-\mathrm{C} 42$ & $0.9(6)$ \\
\hline P1-C22-C27-C26 & $-177.4(3)$ & P3-C40-C41-C42 & $-173.0(3)$ \\
\hline C8-P2-C28-C29 & $-9.5(4)$ & $\mathrm{C} 40-\mathrm{C} 41-\mathrm{C} 42-\mathrm{C} 43$ & $2.1(6)$ \\
\hline C34-P2-C28-C29 & 101.2(4) & $\mathrm{C} 41-\mathrm{C} 42-\mathrm{C} 43-\mathrm{C} 52$ & $-2.6(6)$ \\
\hline $\mathrm{Fe} 1-\mathrm{P} 2-\mathrm{C} 28-\mathrm{C} 29$ & $-135.5(3)$ & $\mathrm{C} 53-\mathrm{C} 44-\mathrm{C} 45-\mathrm{C} 46$ & $-0.7(7)$ \\
\hline C8-P2-C28-C33 & $174.7(3)$ & $\mathrm{C} 44-\mathrm{C} 45-\mathrm{C} 46-\mathrm{C} 47$ & $0.1(7)$ \\
\hline C34-P2-C28-C33 & $-74.6(3)$ & C45-C46-C47-C54 & $-0.2(6)$ \\
\hline Fe1-P2-C28-C33 & $48.7(4)$ & C45-C46-C47-P4 & $174.9(3)$ \\
\hline C33-C28-C29-C30 & $-0.3(6)$ & C73-P4-C47-C46 & $-10.9(4)$ \\
\hline P2-C28-C29-C30 & $-176.0(3)$ & C67-P4-C47-C46 & $98.6(4)$ \\
\hline C28-C29-C30-C31 & $1.2(7)$ & Fe2-P4-C47-C46 & $-131.1(3)$ \\
\hline C29-C30-C31-C32 & $-0.8(7)$ & C73-P4-C47-C54 & $164.2(3)$ \\
\hline C30-C31-C32-C33 & $-0.5(7)$ & C67-P4-C47-C54 & $-86.3(3)$ \\
\hline C31-C32-C33-C28 & $1.4(7)$ & Fe2-P4-C47-C54 & $44.0(3)$ \\
\hline C29-C28-C33-C32 & $-1.0(6)$ & $\mathrm{C} 54-\mathrm{O} 2-\mathrm{C} 51-\mathrm{C} 52$ & $-20.7(5)$ \\
\hline P2-C28-C33-C32 & $175.0(3)$ & $\mathrm{C} 54-\mathrm{O} 2-\mathrm{C} 51-\mathrm{C} 40$ & 159.7(3) \\
\hline C28-P2-C34-C39 & $151.6(3)$ & C41-C40-C51-C52 & $-3.7(6)$ \\
\hline C8-P2-C34-C39 & $-97.1(3)$ & P3-C40-C51-C52 & $170.5(3)$ \\
\hline Fe1-P2-C34-C39 & $22.3(3)$ & $\mathrm{C} 41-\mathrm{C} 40-\mathrm{C} 51-\mathrm{O} 2$ & $175.9(3)$ \\
\hline C28-P2-C34-C35 & $-23.4(4)$ & $\mathrm{P} 3-\mathrm{C} 40-\mathrm{C} 51-\mathrm{O} 2$ & $-9.9(5)$ \\
\hline C8-P2-C34-C35 & $87.8(3)$ & $\mathrm{O} 2-\mathrm{C} 51-\mathrm{C} 52-\mathrm{C} 43$ & $-176.4(3)$ \\
\hline Fe1-P2-C34-C35 & $-152.7(3)$ & C40-C51-C52-C43 & $3.2(6)$ \\
\hline
\end{tabular}




\begin{tabular}{|c|c|c|c|}
\hline $\mathrm{O} 2-\mathrm{C} 51-\mathrm{C} 52-\mathrm{C} 48$ & $3.0(6)$ & C55-C56-C57-C58 & $0.3(6)$ \\
\hline C40-C51-C52-C48 & $-177.4(4)$ & C56-C57-C58-C59 & $-0.1(7)$ \\
\hline C42-C43-C52-C51 & $0.0(6)$ & C57-C58-C59-C60 & $0.3(7)$ \\
\hline $\mathrm{C} 42-\mathrm{C} 43-\mathrm{C} 52-\mathrm{C} 48$ & $-179.3(4)$ & C56-C55-C60-C59 & $0.8(6)$ \\
\hline C53-C48-C52-C51 & $13.2(5)$ & P3-C55-C60-C59 & $-179.5(3)$ \\
\hline C49-C48-C52-C51 & $-106.1(4)$ & C58-C59-C60-C55 & $-0.6(6)$ \\
\hline C50-C48-C52-C51 & $133.2(4)$ & C55-P3-C61-C62 & $-99.8(4)$ \\
\hline $\mathrm{C} 53-\mathrm{C} 48-\mathrm{C} 52-\mathrm{C} 43$ & $-167.5(4)$ & C40-P3-C61-C62 & $9.8(4)$ \\
\hline $\mathrm{C} 49-\mathrm{C} 48-\mathrm{C} 52-\mathrm{C} 43$ & $73.2(5)$ & Fe2-P3-C61-C62 & $136.4(3)$ \\
\hline $\mathrm{C} 50-\mathrm{C} 48-\mathrm{C} 52-\mathrm{C} 43$ & $-47.5(5)$ & C55-P3-C61-C66 & $75.6(4)$ \\
\hline $\mathrm{C} 45-\mathrm{C} 44-\mathrm{C} 53-\mathrm{C} 54$ & $1.2(6)$ & C40-P3-C61-C66 & $-174.7(3)$ \\
\hline $\mathrm{C} 45-\mathrm{C} 44-\mathrm{C} 53-\mathrm{C} 48$ & $-178.0(4)$ & Fe2-P3-C61-C66 & $-48.2(4)$ \\
\hline C52-C48-C53-C54 & $-13.3(5)$ & C66-C61-C62-C63 & $0.4(6)$ \\
\hline C49-C48-C53-C54 & $106.2(4)$ & P3-C61-C62-C63 & $175.8(3)$ \\
\hline C50-C48-C53-C54 & $-134.1(4)$ & C61-C62-C63-C64 & $-0.5(6)$ \\
\hline C52-C48-C53-C44 & $165.9(4)$ & C62-C63-C64-C65 & $-0.1(7)$ \\
\hline $\mathrm{C} 49-\mathrm{C} 48-\mathrm{C} 53-\mathrm{C} 44$ & $-74.7(5)$ & C63-C64-C65-C66 & $0.8(7)$ \\
\hline $\mathrm{C} 50-\mathrm{C} 48-\mathrm{C} 53-\mathrm{C} 44$ & $45.0(5)$ & C64-C65-C66-C61 & $-0.9(7)$ \\
\hline $\mathrm{C} 44-\mathrm{C} 53-\mathrm{C} 54-\mathrm{O} 2$ & $178.0(4)$ & C62-C61-C66-C65 & $0.3(7)$ \\
\hline C48-C53-C54-O2 & $-2.8(6)$ & P3-C61-C66-C65 & $-175.3(4)$ \\
\hline C44-C53-C54-C47 & $-1.3(6)$ & C73-P4-C67-C68 & $93.7(3)$ \\
\hline C48-C53-C54-C47 & $177.9(4)$ & C47-P4-C67-C68 & $-15.0(4)$ \\
\hline C51-O2-C54-C53 & $20.7(5)$ & Fe2-P4-C67-C68 & $-138.9(3)$ \\
\hline $\mathrm{C} 51-\mathrm{O} 2-\mathrm{C} 54-\mathrm{C} 47$ & $-160.0(3)$ & C73-P4-C67-C72 & $-84.1(3)$ \\
\hline $\mathrm{C} 46-\mathrm{C} 47-\mathrm{C} 54-\mathrm{C} 53$ & $0.8(6)$ & C47-P4-C67-C72 & $167.2(3)$ \\
\hline P4-C47-C54-C53 & $-174.6(3)$ & Fe2-P4-C67-C72 & $43.3(3)$ \\
\hline $\mathrm{C} 46-\mathrm{C} 47-\mathrm{C} 54-\mathrm{O} 2$ & $-178.5(3)$ & C72-C67-C68-C69 & $1.0(6)$ \\
\hline $\mathrm{P} 4-\mathrm{C} 47-\mathrm{C} 54-\mathrm{O} 2$ & $6.0(5)$ & P4-C67-C68-C69 & $-176.7(3)$ \\
\hline C61-P3-C55-C60 & $-155.6(3)$ & C67-C68-C69-C70 & $-0.1(6)$ \\
\hline C40-P3-C55-C60 & $93.2(3)$ & C68-C69-C70-C71 & $-0.3(7)$ \\
\hline Fe2-P3-C55-C60 & $-26.6(4)$ & $\mathrm{C} 69-\mathrm{C} 70-\mathrm{C} 71-\mathrm{C} 72$ & $-0.2(7)$ \\
\hline C61-P3-C55-C56 & $24.0(4)$ & C70-C71-C72-C67 & $1.2(7)$ \\
\hline C40-P3-C55-C56 & $-87.2(4)$ & C68-C67-C72-C71 & $-1.6(6)$ \\
\hline Fe2-P3-C55-C56 & $153.0(3)$ & P4-C67-C72-C71 & $176.3(3)$ \\
\hline C60-C55-C56-C57 & $-0.6(6)$ & C67-P4-C73-C78 & $158.3(3)$ \\
\hline P3-C55-C56-C57 & $179.8(3)$ & C47-P4-C73-C78 & $-91.1(3)$ \\
\hline
\end{tabular}




$\begin{array}{lclc}\text { Fe2-P4-C73-C78 } & 26.6(4) & \text { C73-C74-C75-C76 } & 0.2(6) \\ \text { C67-P4-C73-C74 } & -18.9(4) & \text { C74-C75-C76-C77 } & 0.1(7) \\ \text { C47-P4-C73-C74 } & 91.7(4) & \text { C75-C76-C77-C78 } & -1.7(6) \\ \text { Fe2-P4-C73-C74 } & -150.6(3) & \text { C74-C73-C78-C77 } & -2.8(6) \\ \text { C78-C73-C74-C75 } & 1.1(6) & \text { P4-C73-C78-C77 } & 179.9(3) \\ \text { P4-C73-C74-C75 } & 178.2(3) & \text { C76-C77-C78-C73 } & 3.1(6)\end{array}$




\section{$3.5[\mathrm{FeBr}(\mathrm{SciOPP})]_{2}$}

REFERENCE NUMBER: neijk39

CRYSTAL STRUCTURE REPORT

$$
\mathrm{C}_{128} \mathrm{H}_{186} \mathrm{Br}_{2} \mathrm{Fe}_{2} \mathrm{O} \mathrm{P} \mathrm{P}_{4}
$$

or

$[(\mathrm{SciOPP}) \mathrm{Fe}(\mu-\mathrm{Br})]_{2} \cdot \mathrm{Et}_{2} \mathrm{O}$

Report prepared for:

A. Shaps, J. Kneebone, Prof. M. Neidig

February 11, 2015

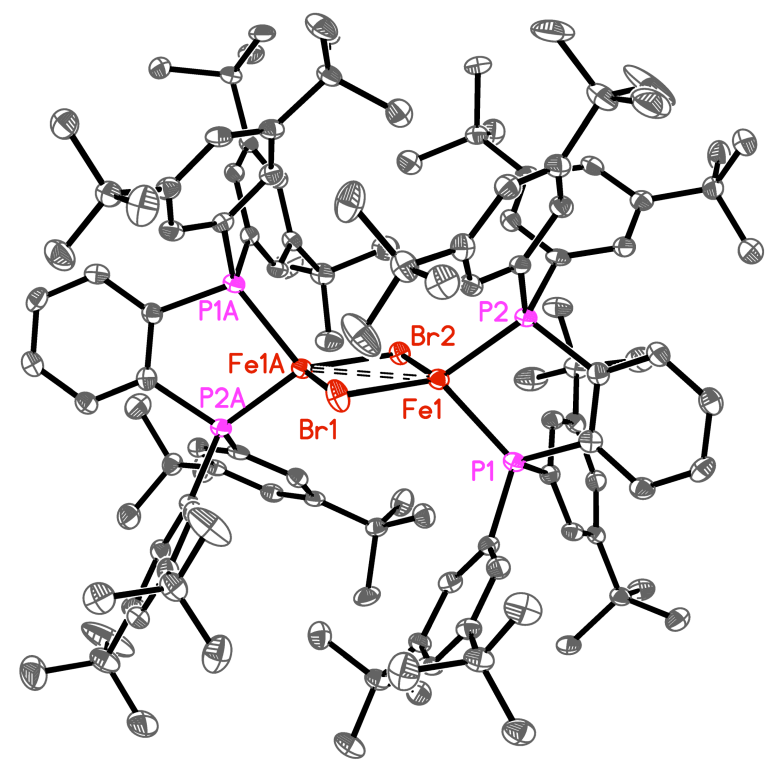

William W. Brennessel

X-ray Crystallographic Facility

Department of Chemistry, University of Rochester

120 Trustee Road

Rochester, NY 14627 


\section{Data collection}

A crystal $\left(0.20 \times 0.18 \times 0.14 \mathrm{~mm}^{3}\right)$ was placed onto the tip of a thin glass optical fiber and mounted on a Bruker SMART APEX II CCD platform diffractometer for a data collection at $100.0(5) \mathrm{K}^{1}$ A preliminary set of cell constants and an orientation matrix were calculated from reflections harvested from three orthogonal wedges of reciprocal space. The full data collection was carried out using MoKa radiation (graphite monochromator) with a frame time of 60 seconds and a detector distance of $4.02 \mathrm{~cm}$. A randomly oriented region of reciprocal space was surveyed: five major sections of frames were collected with $0.50^{\circ}$ steps in $\omega$ at five different $\phi$ settings and a detector position of $-38^{\circ}$ in $2 \theta$. The intensity data were corrected for absorption. ${ }^{2}$ Final cell constants were calculated from the xyz centroids of 4010 strong reflections from the actual data collection after integration. ${ }^{3}$ See Table 1 for additional crystal and refinement information.

\section{Structure solution and refinement}

The structure was solved using SIR20114 and refined using SHELXL-2014/7.5 The space group $P 2 / n$ was determined based on systematic absences and intensity statistics. A direct-methods solution was calculated which provided most non-hydrogen atoms from the E-map. Full-matrix least squares / difference Fourier cycles were performed which located the remaining non-hydrogen atoms. All non-hydrogen atoms were refined with anisotropic displacement parameters. All hydrogen atoms were placed in ideal positions and refined as riding atoms with relative isotropic displacement parameters. The final full matrix least squares refinement converged to $R 1=0.0527$ $\left(F^{2}, I>2 \sigma(I)\right)$ and $w R 2=0.1096\left(F^{2}\right.$, all data $)$.

\section{Structure description}

The structure is the one suggested. The asymmetric unit contains the metal center and one SciOPP ligand in general positions, the two bridging bromido ligands on a crystallographic two-fold axis, and one co-crystallized diethyl ether molecule on a crystallographic inversion center. One tert-butyl group (and its symmetry equivalent) is modeled as disordered over two positions (0.60:0.40) and the diethyl ether molecule is modeled as disordered over the crystallographic inversion center (0.50:0.50).

Unless noted otherwise all structural diagrams containing thermal displacement ellipsoids are drawn at the $50 \%$ probability level.

Data collection, structure solution, and structure refinement were conducted at the X-ray Crystallographic Facility, B51 Hutchison Hall, Department of Chemistry, University of Rochester. All publications arising from this report MUST either 1) include William W. Brennessel as a coauthor or 2) acknowledge William W. Brennessel and the Xray Crystallographic Facility of the Department of Chemistry at the University of Rochester. 
1 APEX3, version 2015.5-2; Bruker AXS: Madison, WI, 2015.

2 Sheldrick, G. M. SADABS, version 2014/5; J. Appl. Cryst. 2015, 48, 3-10.

3 SAINT, version 8.34A; Bruker AXS: Madison, WI, 2013.

4 Burla, M. C.; Caliandro, R.; Camalli, M.; Carrozzini, B.; Cascarano, G. L.; Giacovazzo, C.; Mallamo, M.; Mazzone, A.; Polidori, G.; Spagna, R. SIR2011, version 1.0; J. Appl. Cryst. 2012, 45, 357-361.

5 Sheldrick, G. M. SHELXL-2014/7; Acta. Cryst. 2015, C71, 3-8.

Some equations of interest:

$$
\begin{gathered}
R_{\mathrm{int}}=\Sigma\left|F_{\mathrm{o}}{ }^{2}-<F_{\mathrm{o}}{ }^{2}>\right| / \Sigma\left|F_{\mathrm{o}}{ }^{2}\right| \\
R 1=\Sigma|| F_{\mathrm{o}}|-| F_{\mathrm{c}}|/ \Sigma| F_{\mathrm{o}} \mid \\
w R 2=\left[\Sigma\left[w\left(F_{\mathrm{o}}{ }^{2}-F_{\mathrm{c}}{ }^{2}\right)^{2}\right] / \Sigma\left[w\left(F_{\mathrm{o}}{ }^{2}\right)^{2}\right]\right]^{1 / 2} \\
\text { where } w=1 /\left[\sigma^{2}\left(F_{\mathrm{o}}{ }^{2}\right)+(a P)^{2}+b P\right] \text { and } \\
P=1 / 3 \max \left(0, F_{\mathrm{o}}{ }^{2}\right)+2 / 3 F_{\mathrm{c}}{ }^{2} \\
\text { GOF }=S=\left[\Sigma\left[w\left(F_{\mathrm{o}}{ }^{2}-F_{\mathrm{c}}{ }^{2}\right)^{2}\right] /(m-n)\right]^{1 / 2}
\end{gathered}
$$

where $m=$ number of reflections and $n=$ number of parameters

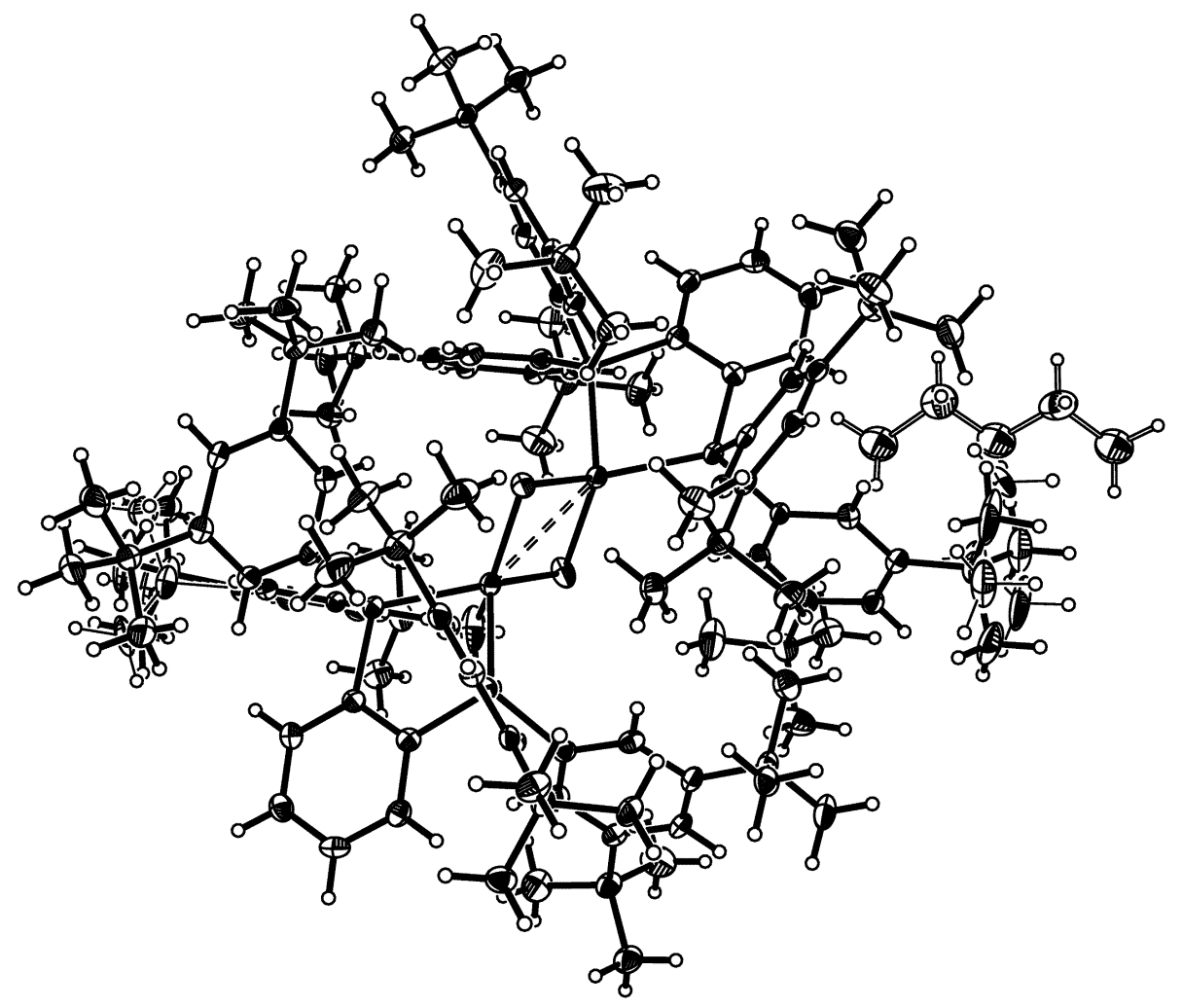



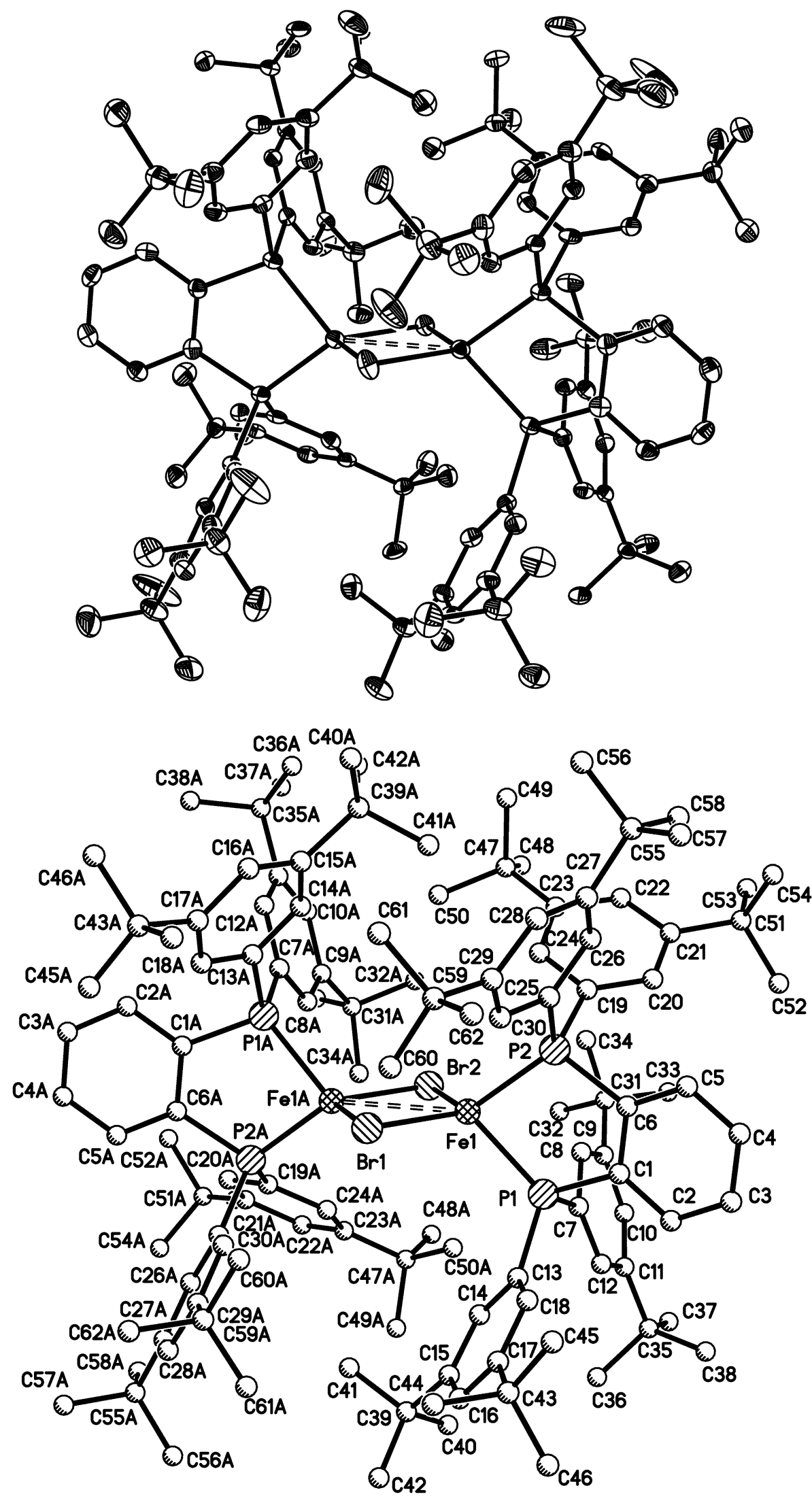

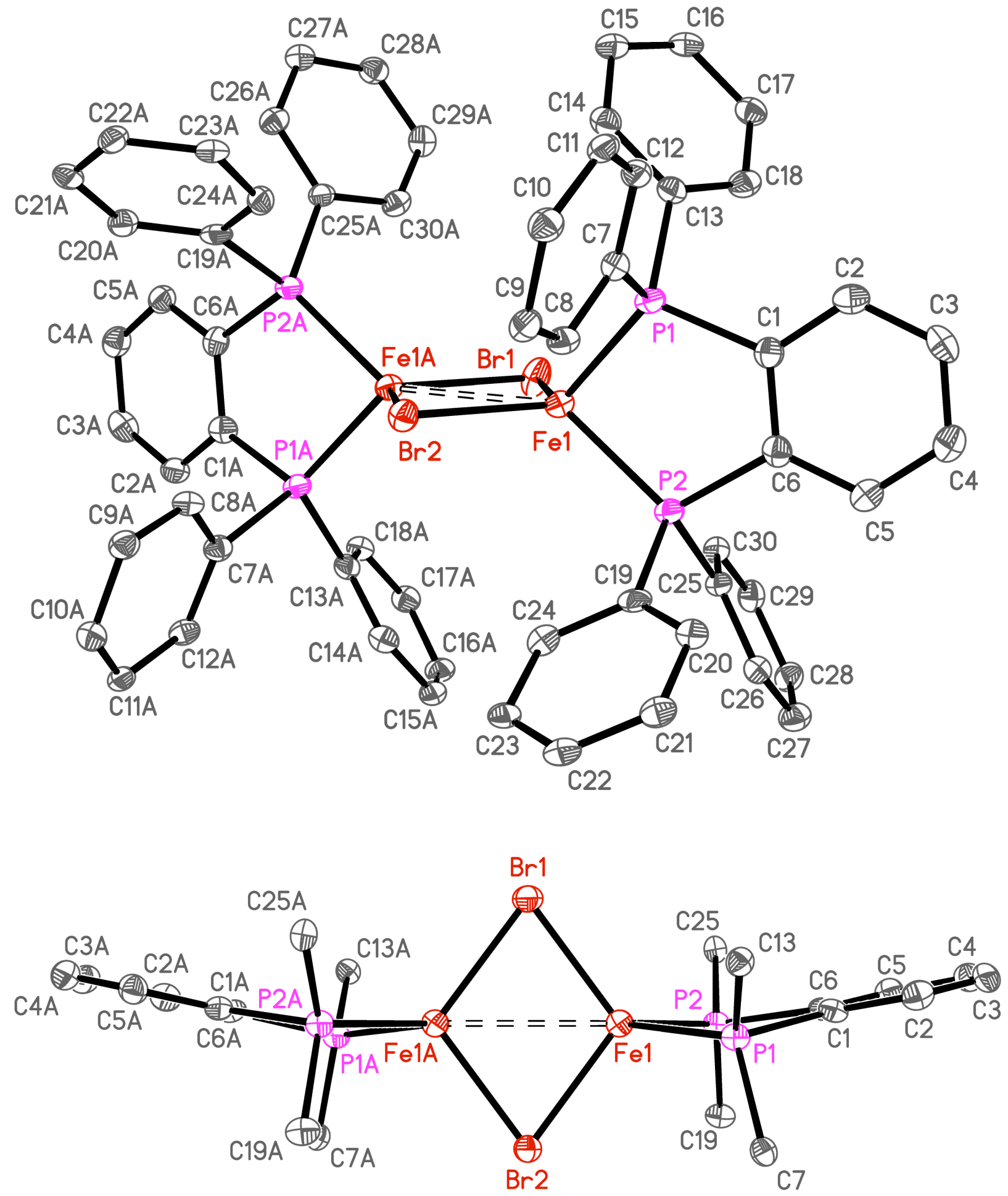
Table S25. Crystal data and structure refinement for neijk39.

\begin{tabular}{|c|c|c|}
\hline Identification code & \multicolumn{2}{|l|}{ neijk39 } \\
\hline Empirical formula & \multicolumn{2}{|c|}{$\mathrm{C} 128 \mathrm{H} 186 \mathrm{Br} 2 \mathrm{Fe} 2 \mathrm{O}$ P4 } \\
\hline Formula weight & \multicolumn{2}{|l|}{2136.16} \\
\hline Temperature & \multicolumn{2}{|l|}{$100.0(5) \mathrm{K}$} \\
\hline Wavelength & \multicolumn{2}{|l|}{$0.71073 \AA$} \\
\hline Crystal system & \multicolumn{2}{|l|}{ monoclinic } \\
\hline Space group & \multicolumn{2}{|l|}{$P 2 / n$} \\
\hline \multirow[t]{3}{*}{ Unit cell dimensions } & $a=14.5951(11) \AA$ & $\alpha=90^{\circ}$ \\
\hline & $b=14.2424(11) \AA$ & $\beta=103.2648(14)^{\circ}$ \\
\hline & $c=30.373(2) \AA$ & $\gamma=90^{\circ}$ \\
\hline Volume & \multicolumn{2}{|l|}{$6145.1(8) \AA^{3}$} \\
\hline$Z$ & \multicolumn{2}{|l|}{2} \\
\hline Density (calculated) & \multicolumn{2}{|l|}{$1.154 \mathrm{Mg} / \mathrm{m}^{3}$} \\
\hline Absorption coefficient & \multicolumn{2}{|l|}{$0.984 \mathrm{~mm}^{-1}$} \\
\hline$F(000)$ & \multicolumn{2}{|l|}{2288} \\
\hline Crystal color, morphology & \multicolumn{2}{|l|}{ dark orange, plate } \\
\hline Crystal size & \multicolumn{2}{|c|}{$0.20 \times 0.18 \times 0.14 \mathrm{~mm}^{3}$} \\
\hline Theta range for data collection & \multicolumn{2}{|l|}{1.986 to $26.409^{\circ}$} \\
\hline Index ranges & \multicolumn{2}{|c|}{$-18 \leq h \leq 18,-17 \leq k \leq 17,-37 \leq l \leq 37$} \\
\hline Reflections collected & \multicolumn{2}{|l|}{74990} \\
\hline Independent reflections & \multicolumn{2}{|c|}{$12610[R($ int $)=0.1312]$} \\
\hline Observed reflections & \multicolumn{2}{|l|}{7743} \\
\hline Completeness to theta $=26.373^{\circ}$ & \multicolumn{2}{|l|}{$100.0 \%$} \\
\hline Absorption correction & \multicolumn{2}{|l|}{ Multi-scan } \\
\hline Max. and min. transmission & \multicolumn{2}{|l|}{0.7454 and 0.6548} \\
\hline Refinement method & \multicolumn{2}{|c|}{ Full-matrix least-squares on $F^{2}$} \\
\hline Data / restraints / parameters & \multicolumn{2}{|l|}{$12610 / 46 / 677$} \\
\hline Goodness-of-fit on $F^{2}$ & \multicolumn{2}{|l|}{1.021} \\
\hline Final $R$ indices $[I>2 \operatorname{sigma}(I)]$ & \multicolumn{2}{|c|}{$R 1=0.0527, w R 2=0.0932$} \\
\hline$R$ indices (all data) & \multicolumn{2}{|c|}{$R 1=0.1085, w R 2=0.1096$} \\
\hline Largest diff. peak and hole & \multicolumn{2}{|c|}{0.448 and -0.421 e. $\AA^{-3}$} \\
\hline
\end{tabular}


Table S26. Atomic coordinates $\left(\times 10^{4}\right)$ and equivalent isotropic displacement parameters $\left(\AA^{2} \times 10^{3}\right)$ for neijk39. $U_{e q}$ is defined as one third of the trace of the orthogonalized $U_{i j}$ tensor.

\begin{tabular}{|c|c|c|c|c|}
\hline & $\mathrm{x}$ & $\mathrm{y}$ & $\mathrm{z}$ & $\mathrm{U}_{\mathrm{eq}}$ \\
\hline $\mathrm{Fe} 1$ & $3248(1)$ & 1841(1) & $2254(1)$ & $17(1)$ \\
\hline $\mathrm{Br} 1$ & 2500 & $3216(1)$ & 2500 & $27(1)$ \\
\hline $\mathrm{Br} 2$ & 2500 & $457(1)$ & 2500 & $20(1)$ \\
\hline P1 & $4835(1)$ & 1698(1) & $2476(1)$ & $17(1)$ \\
\hline $\mathrm{P} 2$ & $3496(1)$ & $1836(1)$ & $1540(1)$ & $16(1)$ \\
\hline $\mathrm{C} 1$ & $5366(2)$ & 1978(2) & $2000(1)$ & $18(1)$ \\
\hline $\mathrm{C} 2$ & $6328(2)$ & $2139(2)$ & $2046(1)$ & $21(1)$ \\
\hline C3 & $6689(3)$ & 2335(2) & 1672(1) & $24(1)$ \\
\hline $\mathrm{C} 4$ & 6093(3) & $2360(2)$ & $1246(1)$ & $24(1)$ \\
\hline C5 & $5141(3)$ & 2201(2) & 1195(1) & $21(1)$ \\
\hline C6 & $4765(2)$ & 2003(2) & $1568(1)$ & $18(1)$ \\
\hline C7 & $5317(2)$ & $511(2)$ & $2625(1)$ & $17(1)$ \\
\hline $\mathrm{C} 8$ & $4766(2)$ & $-224(2)$ & 2401(1) & $18(1)$ \\
\hline C9 & $5028(2)$ & $-1155(2)$ & 2484(1) & 19(1) \\
\hline $\mathrm{C} 10$ & $5881(2)$ & $-1327(2)$ & $2802(1)$ & $20(1)$ \\
\hline C11 & $6459(2)$ & $-610(2)$ & $3022(1)$ & $17(1)$ \\
\hline $\mathrm{C} 12$ & $6159(2)$ & $314(2)$ & $2927(1)$ & $17(1)$ \\
\hline C13 & $5436(2)$ & 2463(2) & 2941(1) & $17(1)$ \\
\hline $\mathrm{C} 14$ & $5445(2)$ & 2212(2) & $3379(1)$ & $19(1)$ \\
\hline C15 & $5760(2)$ & 2838(2) & 3743(1) & 19(1) \\
\hline $\mathrm{C} 16$ & $6071(2)$ & 3711(2) & $3642(1)$ & $21(1)$ \\
\hline C17 & $6057(2)$ & $4000(2)$ & 3201(1) & $20(1)$ \\
\hline $\mathrm{C} 18$ & $5737(2)$ & $3365(2)$ & $2852(1)$ & $21(1)$ \\
\hline C19 & $3161(2)$ & $742(2)$ & 1223(1) & $17(1)$ \\
\hline $\mathrm{C} 20$ & $3697(2)$ & $265(2)$ & $972(1)$ & $20(1)$ \\
\hline $\mathrm{C} 21$ & $3367(2)$ & $-556(2)$ & $734(1)$ & $19(1)$ \\
\hline $\mathrm{C} 22$ & $2473(2)$ & $-872(2)$ & $755(1)$ & $20(1)$ \\
\hline $\mathrm{C} 23$ & 1914(2) & $-423(2)$ & $1007(1)$ & $18(1)$ \\
\hline $\mathrm{C} 24$ & $2281(2)$ & $382(2)$ & 1241(1) & $18(1)$ \\
\hline $\mathrm{C} 25$ & $2976(2)$ & 2729(2) & $1120(1)$ & $17(1)$ \\
\hline C26 & $2803(2)$ & $2580(2)$ & $658(1)$ & 19(1) \\
\hline
\end{tabular}




\begin{tabular}{|c|c|c|c|c|}
\hline $\mathrm{C} 27$ & $2440(2)$ & $3293(3)$ & $350(1)$ & $22(1)$ \\
\hline $\mathrm{C} 28$ & $2234(2)$ & $4146(2)$ & $525(1)$ & $21(1)$ \\
\hline $\mathrm{C} 29$ & $2383(2)$ & 4323(2) & $988(1)$ & $21(1)$ \\
\hline $\mathrm{C} 30$ & $2758(2)$ & $3599(2)$ & 1281(1) & $19(1)$ \\
\hline $\mathrm{C} 31$ & $4423(3)$ & $-1974(2)$ & $2255(1)$ & $23(1)$ \\
\hline $\mathrm{C} 32$ & $4046(3)$ & $-2510(3)$ & $2618(1)$ & $37(1)$ \\
\hline $\mathrm{C} 33$ & $5011(3)$ & $-2630(3)$ & $2035(2)$ & $38(1)$ \\
\hline $\mathrm{C} 34$ & $3573(3)$ & $-1643(2)$ & $1895(1)$ & $29(1)$ \\
\hline $\mathrm{C} 35$ & $7403(2)$ & $-810(2)$ & $3354(1)$ & 19(1) \\
\hline $\mathrm{C} 36$ & 7391(3) & $-363(3)$ & $3814(1)$ & $29(1)$ \\
\hline $\mathrm{C} 37$ & 7601(3) & $-1861(3)$ & $3424(1)$ & $28(1)$ \\
\hline $\mathrm{C} 38$ & $8200(2)$ & $-371(3)$ & $3172(1)$ & $25(1)$ \\
\hline C39 & $5727(3)$ & $2536(2)$ & $4227(1)$ & $22(1)$ \\
\hline $\mathrm{C} 40$ & $6305(3)$ & 1633(2) & $4345(1)$ & $25(1)$ \\
\hline C41 & $4698(3)$ & 2341(3) & $4244(1)$ & $28(1)$ \\
\hline $\mathrm{C} 42$ & $6124(3)$ & 3293(3) & $4577(1)$ & $32(1)$ \\
\hline $\mathrm{C} 43$ & $6412(3)$ & 4983(2) & $3122(1)$ & $24(1)$ \\
\hline C44 & $5835(3)$ & 5715(3) & $3315(2)$ & $38(1)$ \\
\hline $\mathrm{C} 45$ & $6313(3)$ & 5182(3) & 2621(1) & $36(1)$ \\
\hline C46 & $7460(3)$ & $5065(3)$ & $3365(1)$ & $31(1)$ \\
\hline C47 & $936(2)$ & $-807(2)$ & 1014(1) & $20(1)$ \\
\hline $\mathrm{C} 48$ & $964(3)$ & $-1877(3)$ & 1081(1) & $30(1)$ \\
\hline C49 & $268(3)$ & $-571(3)$ & $555(1)$ & $29(1)$ \\
\hline $\mathrm{C} 50$ & $548(3)$ & $-370(3)$ & $1396(1)$ & $26(1)$ \\
\hline $\mathrm{C} 51$ & $3945(3)$ & $-1059(2)$ & $444(1)$ & $22(1)$ \\
\hline C52 & 4987(3) & $-1071(3)$ & $680(1)$ & $33(1)$ \\
\hline C53 & $3632(3)$ & $-2085(3)$ & $342(1)$ & $33(1)$ \\
\hline C54 & $3807(3)$ & $-532(3)$ & $-9(1)$ & $34(1)$ \\
\hline C55 & $2213(5)$ & $3140(5)$ & $-162(2)$ & $30(1)$ \\
\hline C56 & $1158(4)$ & $3285(6)$ & $-365(2)$ & $50(2)$ \\
\hline C57 & $2770(6)$ & $3854(6)$ & $-371(2)$ & $54(2)$ \\
\hline C58 & $2466(8)$ & $2142(5)$ & $-277(2)$ & $77(3)$ \\
\hline C55' & $2357(6)$ & $3125(7)$ & $-156(2)$ & $30(1)$ \\
\hline C56' & $3290(6)$ & 2796(9) & $-254(3)$ & $46(3)$ \\
\hline C57' & $1610(8)$ & 2363(9) & $-314(4)$ & $54(2)$ \\
\hline C58' & $2040(11)$ & 4009(8) & $-439(4)$ & $77(3)$ \\
\hline
\end{tabular}




\begin{tabular}{lllrl} 
C59 & $2106(3)$ & $5276(3)$ & $1149(1)$ & $27(1)$ \\
C60 & $2326(4)$ & $5350(3)$ & $1667(1)$ & $49(1)$ \\
C61 & $1046(3)$ & $5434(3)$ & $967(2)$ & $39(1)$ \\
C62 & $2648(3)$ & $6055(3)$ & $972(1)$ & $32(1)$ \\
C63 & $5014(16)$ & $5391(10)$ & $-731(5)$ & $53(3)$ \\
C64 & $5354(7)$ & $4663(6)$ & $-347(3)$ & $50(3)$ \\
O1 & $5040(20)$ & $5030(30)$ & $44(5)$ & $53(3)$ \\
C65 & $5388(7)$ & $4392(6)$ & $430(3)$ & $48(2)$ \\
C66 & $5002(16)$ & $4844(11)$ & $820(5)$ & $53(3)$ \\
\hline
\end{tabular}


Table S27. Bond lengths $[\AA]$ and angles $\left[{ }^{\circ}\right]$ for neijk39.

\begin{tabular}{|c|c|c|c|}
\hline $\mathrm{Fe}(1)-\mathrm{P}(1)$ & $2.2681(10)$ & $C(13)-C(14)$ & $1.377(5)$ \\
\hline $\mathrm{Fe}(1)-\mathrm{P}(2)$ & $2.2791(9)$ & $C(13)-C(18)$ & $1.404(5)$ \\
\hline $\mathrm{Fe}(1)-\operatorname{Br}(1)$ & $2.4411(7)$ & $C(14)-C(15)$ & $1.412(5)$ \\
\hline $\mathrm{Fe}(1)-\operatorname{Br}(2)$ & $2.4518(7)$ & $\mathrm{C}(14)-\mathrm{H}(14 \mathrm{~A})$ & 0.9500 \\
\hline $\mathrm{Fe}(1)-\mathrm{Fe}(1) \# 1$ & $2.9160(9)$ & $C(15)-C(16)$ & $1.382(5)$ \\
\hline $\operatorname{Br}(1)-\mathrm{Fe}(1) \# 1$ & $2.4410(7)$ & $C(15)-C(39)$ & $1.543(5)$ \\
\hline $\operatorname{Br}(2)-\mathrm{Fe}(1) \# 1$ & $2.4517(7)$ & $C(16)-C(17)$ & $1.398(5)$ \\
\hline $\mathrm{P}(1)-\mathrm{C}(1)$ & $1.834(3)$ & $\mathrm{C}(16)-\mathrm{H}(16 \mathrm{~A})$ & 0.9500 \\
\hline $\mathrm{P}(1)-\mathrm{C}(13)$ & $1.839(3)$ & $C(17)-C(18)$ & $1.389(5)$ \\
\hline $\mathrm{P}(1)-\mathrm{C}(7)$ & $1.846(3)$ & $\mathrm{C}(17)-\mathrm{C}(43)$ & $1.530(5)$ \\
\hline $\mathrm{P}(2)-\mathrm{C}(25)$ & $1.836(3)$ & $\mathrm{C}(18)-\mathrm{H}(18 \mathrm{~A})$ & 0.9500 \\
\hline $\mathrm{P}(2)-\mathrm{C}(19)$ & $1.838(3)$ & $C(19)-C(20)$ & $1.389(5)$ \\
\hline $\mathrm{P}(2)-\mathrm{C}(6)$ & $1.850(3)$ & $\mathrm{C}(19)-\mathrm{C}(24)$ & $1.396(5)$ \\
\hline $\mathrm{C}(1)-\mathrm{C}(2)$ & $1.398(5)$ & $\mathrm{C}(20)-\mathrm{C}(21)$ & $1.401(5)$ \\
\hline$C(1)-C(6)$ & $1.401(4)$ & $\mathrm{C}(20)-\mathrm{H}(20 \mathrm{~A})$ & 0.9500 \\
\hline $\mathrm{C}(2)-\mathrm{C}(3)$ & $1.388(5)$ & $C(21)-C(22)$ & $1.395(5)$ \\
\hline $\mathrm{C}(2)-\mathrm{H}(2 \mathrm{~A})$ & 0.9500 & $\mathrm{C}(21)-\mathrm{C}(51)$ & $1.530(5)$ \\
\hline$C(3)-C(4)$ & $1.384(5)$ & $C(22)-C(23)$ & $1.397(5)$ \\
\hline $\mathrm{C}(3)-\mathrm{H}(3 \mathrm{~A})$ & 0.9500 & $\mathrm{C}(22)-\mathrm{H}(22 \mathrm{~A})$ & 0.9500 \\
\hline$C(4)-C(5)$ & $1.382(5)$ & $C(23)-C(24)$ & $1.389(5)$ \\
\hline $\mathrm{C}(4)-\mathrm{H}(4 \mathrm{~A})$ & 0.9500 & $\mathrm{C}(23)-\mathrm{C}(47)$ & $1.534(5)$ \\
\hline$C(5)-C(6)$ & $1.394(5)$ & $\mathrm{C}(24)-\mathrm{H}(24 \mathrm{~A})$ & 0.9500 \\
\hline $\mathrm{C}(5)-\mathrm{H}(5 \mathrm{~A})$ & 0.9500 & $C(25)-C(26)$ & $1.386(4)$ \\
\hline$C(7)-C(12)$ & $1.384(4)$ & $\mathrm{C}(25)-\mathrm{C}(30)$ & $1.395(5)$ \\
\hline$C(7)-C(8)$ & $1.399(5)$ & $C(26)-C(27)$ & $1.400(5)$ \\
\hline $\mathrm{C}(8)-\mathrm{C}(9)$ & $1.387(5)$ & $\mathrm{C}(26)-\mathrm{H}(26 \mathrm{~A})$ & 0.9500 \\
\hline $\mathrm{C}(8)-\mathrm{H}(8 \mathrm{~A})$ & 0.9500 & $\mathrm{C}(27)-\mathrm{C}(28)$ & $1.386(5)$ \\
\hline$C(9)-C(10)$ & $1.410(5)$ & $C(27)-C(55)$ & $1.530(6)$ \\
\hline $\mathrm{C}(9)-\mathrm{C}(31)$ & $1.531(5)$ & $\mathrm{C}(27)-\mathrm{C}\left(55^{\prime}\right)$ & $1.533(7)$ \\
\hline$C(10)-C(11)$ & $1.394(5)$ & $C(28)-C(29)$ & $1.395(5)$ \\
\hline $\mathrm{C}(10)-\mathrm{H}(10 \mathrm{~A})$ & 0.9500 & $\mathrm{C}(28)-\mathrm{H}(28 \mathrm{~A})$ & 0.9500 \\
\hline$C(11)-C(12)$ & $1.397(5)$ & $\mathrm{C}(29)-\mathrm{C}(30)$ & $1.390(5)$ \\
\hline$C(11)-C(35)$ & $1.536(4)$ & $C(29)-C(59)$ & $1.528(5)$ \\
\hline $\mathrm{C}(12)-\mathrm{H}(12 \mathrm{~A})$ & 0.9500 & $\mathrm{C}(30)-\mathrm{H}(30 \mathrm{~A})$ & 0.9500 \\
\hline
\end{tabular}




\begin{tabular}{|c|c|c|c|}
\hline$C(31)-C(33)$ & $1.522(5)$ & $\mathrm{C}(43)-\mathrm{C}(45)$ & $1.523(5)$ \\
\hline$C(31)-C(34)$ & $1.528(5)$ & $\mathrm{C}(43)-\mathrm{C}(44)$ & $1.539(5)$ \\
\hline$C(31)-C(32)$ & $1.542(5)$ & $C(43)-C(46)$ & $1.543(5)$ \\
\hline $\mathrm{C}(32)-\mathrm{H}(32 \mathrm{~A})$ & 0.9800 & $\mathrm{C}(44)-\mathrm{H}(44 \mathrm{~A})$ & 0.9800 \\
\hline $\mathrm{C}(32)-\mathrm{H}(32 \mathrm{~B})$ & 0.9800 & $\mathrm{C}(44)-\mathrm{H}(44 \mathrm{~B})$ & 0.9800 \\
\hline $\mathrm{C}(32)-\mathrm{H}(32 \mathrm{C})$ & 0.9800 & $\mathrm{C}(44)-\mathrm{H}(44 \mathrm{C})$ & 0.9800 \\
\hline $\mathrm{C}(33)-\mathrm{H}(33 \mathrm{~A})$ & 0.9800 & $\mathrm{C}(45)-\mathrm{H}(45 \mathrm{~A})$ & 0.9800 \\
\hline $\mathrm{C}(33)-\mathrm{H}(33 \mathrm{~B})$ & 0.9800 & $\mathrm{C}(45)-\mathrm{H}(45 \mathrm{~B})$ & 0.9800 \\
\hline $\mathrm{C}(33)-\mathrm{H}(33 \mathrm{C})$ & 0.9800 & $\mathrm{C}(45)-\mathrm{H}(45 \mathrm{C})$ & 0.9800 \\
\hline $\mathrm{C}(34)-\mathrm{H}(34 \mathrm{~A})$ & 0.9800 & $\mathrm{C}(46)-\mathrm{H}(46 \mathrm{~A})$ & 0.9800 \\
\hline $\mathrm{C}(34)-\mathrm{H}(34 \mathrm{~B})$ & 0.9800 & $\mathrm{C}(46)-\mathrm{H}(46 \mathrm{~B})$ & 0.9800 \\
\hline $\mathrm{C}(34)-\mathrm{H}(34 \mathrm{C})$ & 0.9800 & $\mathrm{C}(46)-\mathrm{H}(46 \mathrm{C})$ & 0.9800 \\
\hline$C(35)-C(37)$ & $1.529(5)$ & $C(47)-C(50)$ & $1.532(5)$ \\
\hline$C(35)-C(38)$ & $1.533(5)$ & $\mathrm{C}(47)-\mathrm{C}(48)$ & $1.536(5)$ \\
\hline$C(35)-C(36)$ & $1.538(5)$ & $\mathrm{C}(47)-\mathrm{C}(49)$ & $1.544(5)$ \\
\hline $\mathrm{C}(36)-\mathrm{H}(36 \mathrm{~A})$ & 0.9800 & $\mathrm{C}(48)-\mathrm{H}(48 \mathrm{~A})$ & 0.9800 \\
\hline $\mathrm{C}(36)-\mathrm{H}(36 \mathrm{~B})$ & 0.9800 & $\mathrm{C}(48)-\mathrm{H}(48 \mathrm{~B})$ & 0.9800 \\
\hline $\mathrm{C}(36)-\mathrm{H}(36 \mathrm{C})$ & 0.9800 & $\mathrm{C}(48)-\mathrm{H}(48 \mathrm{C})$ & 0.9800 \\
\hline $\mathrm{C}(37)-\mathrm{H}(37 \mathrm{~A})$ & 0.9800 & $\mathrm{C}(49)-\mathrm{H}(49 \mathrm{~A})$ & 0.9800 \\
\hline C(37)-H(37B) & 0.9800 & $\mathrm{C}(49)-\mathrm{H}(49 \mathrm{~B})$ & 0.9800 \\
\hline $\mathrm{C}(37)-\mathrm{H}(37 \mathrm{C})$ & 0.9800 & $\mathrm{C}(49)-\mathrm{H}(49 \mathrm{C})$ & 0.9800 \\
\hline $\mathrm{C}(38)-\mathrm{H}(38 \mathrm{~A})$ & 0.9800 & $\mathrm{C}(50)-\mathrm{H}(50 \mathrm{~A})$ & 0.9800 \\
\hline $\mathrm{C}(38)-\mathrm{H}(38 \mathrm{~B})$ & 0.9800 & $\mathrm{C}(50)-\mathrm{H}(50 \mathrm{~B})$ & 0.9800 \\
\hline $\mathrm{C}(38)-\mathrm{H}(38 \mathrm{C})$ & 0.9800 & $\mathrm{C}(50)-\mathrm{H}(50 \mathrm{C})$ & 0.9800 \\
\hline$C(39)-C(42)$ & $1.532(5)$ & $\mathrm{C}(51)-\mathrm{C}(52)$ & $1.525(5)$ \\
\hline $\mathrm{C}(39)-\mathrm{C}(40)$ & $1.535(5)$ & $\mathrm{C}(51)-\mathrm{C}(54)$ & $1.540(5)$ \\
\hline $\mathrm{C}(39)-\mathrm{C}(41)$ & $1.540(5)$ & $C(51)-C(53)$ & $1.541(5)$ \\
\hline $\mathrm{C}(40)-\mathrm{H}(40 \mathrm{~A})$ & 0.9800 & $\mathrm{C}(52)-\mathrm{H}(52 \mathrm{~A})$ & 0.9800 \\
\hline $\mathrm{C}(40)-\mathrm{H}(40 \mathrm{~B})$ & 0.9800 & $\mathrm{C}(52)-\mathrm{H}(52 \mathrm{~B})$ & 0.9800 \\
\hline $\mathrm{C}(40)-\mathrm{H}(40 \mathrm{C})$ & 0.9800 & $\mathrm{C}(52)-\mathrm{H}(52 \mathrm{C})$ & 0.9800 \\
\hline $\mathrm{C}(41)-\mathrm{H}(41 \mathrm{~A})$ & 0.9800 & $\mathrm{C}(53)-\mathrm{H}(53 \mathrm{~A})$ & 0.9800 \\
\hline $\mathrm{C}(41)-\mathrm{H}(41 \mathrm{~B})$ & 0.9800 & $\mathrm{C}(53)-\mathrm{H}(53 \mathrm{~B})$ & 0.9800 \\
\hline $\mathrm{C}(41)-\mathrm{H}(41 \mathrm{C})$ & 0.9800 & $\mathrm{C}(53)-\mathrm{H}(53 \mathrm{C})$ & 0.9800 \\
\hline $\mathrm{C}(42)-\mathrm{H}(42 \mathrm{~A})$ & 0.9800 & $\mathrm{C}(54)-\mathrm{H}(54 \mathrm{~A})$ & 0.9800 \\
\hline $\mathrm{C}(42)-\mathrm{H}(42 \mathrm{~B})$ & 0.9800 & $\mathrm{C}(54)-\mathrm{H}(54 \mathrm{~B})$ & 0.9800 \\
\hline $\mathrm{C}(42)-\mathrm{H}(42 \mathrm{C})$ & 0.9800 & $\mathrm{C}(54)-\mathrm{H}(54 \mathrm{C})$ & 0.9800 \\
\hline
\end{tabular}




\begin{tabular}{|c|c|c|c|}
\hline$C(55)-C(57)$ & $1.529(6)$ & $C(63)-C(64)$ & $1.556(13)$ \\
\hline$C(55)-C(58)$ & $1.530(6)$ & $\mathrm{C}(63)-\mathrm{H}(63 \mathrm{~A})$ & 0.9800 \\
\hline$C(55)-C(56)$ & $1.535(6)$ & $\mathrm{C}(63)-\mathrm{H}(63 \mathrm{~B})$ & 0.9800 \\
\hline $\mathrm{C}(56)-\mathrm{H}(56 \mathrm{~A})$ & 0.9800 & $\mathrm{C}(63)-\mathrm{H}(63 \mathrm{C})$ & 0.9800 \\
\hline $\mathrm{C}(56)-\mathrm{H}(56 \mathrm{~B})$ & 0.9800 & $\mathrm{C}(64)-\mathrm{O}(1)$ & $1.46(3)$ \\
\hline $\mathrm{C}(56)-\mathrm{H}(56 \mathrm{C})$ & 0.9800 & $\mathrm{C}(64)-\mathrm{H}(64 \mathrm{~A})$ & 0.9900 \\
\hline C(57)-H(57A) & 0.9800 & $\mathrm{C}(64)-\mathrm{H}(64 \mathrm{~B})$ & 0.9900 \\
\hline $\mathrm{C}(57)-\mathrm{H}(57 \mathrm{~B})$ & 0.9800 & $\mathrm{O}(1)-\mathrm{C}(65)$ & $1.47(2)$ \\
\hline $\mathrm{C}(57)-\mathrm{H}(57 \mathrm{C})$ & 0.9800 & $C(65)-C(66)$ & $1.562(13)$ \\
\hline $\mathrm{C}(58)-\mathrm{H}(58 \mathrm{~A})$ & 0.9800 & $\mathrm{C}(65)-\mathrm{H}(65 \mathrm{~A})$ & 0.9900 \\
\hline $\mathrm{C}(58)-\mathrm{H}(58 \mathrm{~B})$ & 0.9800 & $\mathrm{C}(65)-\mathrm{H}(65 \mathrm{~B})$ & 0.9900 \\
\hline $\mathrm{C}(58)-\mathrm{H}(58 \mathrm{C})$ & 0.9800 & $\mathrm{C}(66)-\mathrm{H}(66 \mathrm{~A})$ & 0.9800 \\
\hline $\mathrm{C}\left(55^{\prime}\right)-\mathrm{C}\left(56^{\prime}\right)$ & $1.531(7)$ & $\mathrm{C}(66)-\mathrm{H}(66 \mathrm{~B})$ & 0.9800 \\
\hline$C\left(55^{\prime}\right)-C\left(58^{\prime}\right)$ & $1.534(7)$ & $\mathrm{C}(66)-\mathrm{H}(66 \mathrm{C})$ & 0.9800 \\
\hline $\mathrm{C}\left(55^{\prime}\right)-\mathrm{C}\left(57^{\prime}\right)$ & $1.535(7)$ & $\mathrm{P}(1)-\mathrm{Fe}(1)-\mathrm{P}(2)$ & $84.74(3)$ \\
\hline $\mathrm{C}\left(56^{\prime}\right)-\mathrm{H}(56 \mathrm{D})$ & 0.9800 & $\mathrm{P}(1)-\mathrm{Fe}(1)-\mathrm{Br}(1)$ & $118.65(3)$ \\
\hline $\mathrm{C}\left(56^{\prime}\right)-\mathrm{H}(56 \mathrm{E})$ & 0.9800 & $\mathrm{P}(2)-\mathrm{Fe}(1)-\mathrm{Br}(1)$ & $118.27(3)$ \\
\hline $\mathrm{C}\left(56^{\prime}\right)-\mathrm{H}(56 \mathrm{~F})$ & 0.9800 & $\mathrm{P}(1)-\mathrm{Fe}(1)-\mathrm{Br}(2)$ & $109.45(3)$ \\
\hline $\mathrm{C}\left(57^{\prime}\right)-\mathrm{H}(57 \mathrm{D})$ & 0.9800 & $\mathrm{P}(2)-\mathrm{Fe}(1)-\mathrm{Br}(2)$ & $117.81(3)$ \\
\hline $\mathrm{C}\left(57^{\prime}\right)-\mathrm{H}(57 \mathrm{E})$ & 0.9800 & $\operatorname{Br}(1)-\mathrm{Fe}(1)-\mathrm{Br}(2)$ & $106.83(2)$ \\
\hline $\mathrm{C}\left(57^{\prime}\right)-\mathrm{H}(57 \mathrm{~F})$ & 0.9800 & $\mathrm{P}(1)-\mathrm{Fe}(1)-\mathrm{Fe}(1) \# 1$ & $132.98(3)$ \\
\hline $\mathrm{C}\left(58^{\prime}\right)-\mathrm{H}(58 \mathrm{D})$ & 0.9800 & $\mathrm{P}(2)-\mathrm{Fe}(1)-\mathrm{Fe}(1) \# 1$ & $142.07(4)$ \\
\hline $\mathrm{C}\left(58^{\prime}\right)-\mathrm{H}(58 \mathrm{E})$ & 0.9800 & $\operatorname{Br}(1)-\mathrm{Fe}(1)-\mathrm{Fe}(1) \# 1$ & $53.323(14)$ \\
\hline $\mathrm{C}\left(58^{\prime}\right)-\mathrm{H}(58 \mathrm{~F})$ & 0.9800 & $\operatorname{Br}(2)-\mathrm{Fe}(1)-\mathrm{Fe}(1) \# 1$ & $53.510(14)$ \\
\hline $\mathrm{C}(59)-\mathrm{C}(62)$ & $1.528(5)$ & $\mathrm{Fe}(1) \# 1-\mathrm{Br}(1)-\mathrm{Fe}(1)$ & $73.35(3)$ \\
\hline$C(59)-C(60)$ & $1.535(5)$ & $\mathrm{Fe}(1) \# 1-\mathrm{Br}(2)-\mathrm{Fe}(1)$ & $72.98(3)$ \\
\hline$C(59)-C(61)$ & $1.535(5)$ & $C(1)-P(1)-C(13)$ & $105.24(15)$ \\
\hline $\mathrm{C}(60)-\mathrm{H}(60 \mathrm{~A})$ & 0.9800 & $\mathrm{C}(1)-\mathrm{P}(1)-\mathrm{C}(7)$ & $100.81(15)$ \\
\hline $\mathrm{C}(60)-\mathrm{H}(60 \mathrm{~B})$ & 0.9800 & $\mathrm{C}(13)-\mathrm{P}(1)-\mathrm{C}(7)$ & $105.44(15)$ \\
\hline $\mathrm{C}(60)-\mathrm{H}(60 \mathrm{C})$ & 0.9800 & $\mathrm{C}(1)-\mathrm{P}(1)-\mathrm{Fe}(1)$ & $109.46(11)$ \\
\hline $\mathrm{C}(61)-\mathrm{H}(61 \mathrm{~A})$ & 0.9800 & $\mathrm{C}(13)-\mathrm{P}(1)-\mathrm{Fe}(1)$ & $116.70(11)$ \\
\hline $\mathrm{C}(61)-\mathrm{H}(61 \mathrm{~B})$ & 0.9800 & $\mathrm{C}(7)-\mathrm{P}(1)-\mathrm{Fe}(1)$ & $117.43(11)$ \\
\hline $\mathrm{C}(61)-\mathrm{H}(61 \mathrm{C})$ & 0.9800 & $C(25)-P(2)-C(19)$ & $101.86(15)$ \\
\hline $\mathrm{C}(62)-\mathrm{H}(62 \mathrm{~A})$ & 0.9800 & $\mathrm{C}(25)-\mathrm{P}(2)-\mathrm{C}(6)$ & $101.13(15)$ \\
\hline $\mathrm{C}(62)-\mathrm{H}(62 \mathrm{~B})$ & 0.9800 & $C(19)-P(2)-C(6)$ & $106.00(15)$ \\
\hline $\mathrm{C}(62)-\mathrm{H}(62 \mathrm{C})$ & 0.9800 & $\mathrm{C}(25)-\mathrm{P}(2)-\mathrm{Fe}(1)$ & $121.84(11)$ \\
\hline
\end{tabular}




\begin{tabular}{|c|c|c|c|}
\hline $\mathrm{C}(19)-\mathrm{P}(2)-\mathrm{Fe}(1)$ & $114.85(11)$ & $\mathrm{C}(7)-\mathrm{C}(12)-\mathrm{H}(12 \mathrm{~A})$ & 119.4 \\
\hline $\mathrm{C}(6)-\mathrm{P}(2)-\mathrm{Fe}(1)$ & $109.38(11)$ & $\mathrm{C}(11)-\mathrm{C}(12)-\mathrm{H}(12 \mathrm{~A})$ & 119.4 \\
\hline $\mathrm{C}(2)-\mathrm{C}(1)-\mathrm{C}(6)$ & 119.3(3) & $\mathrm{C}(14)-\mathrm{C}(13)-\mathrm{C}(18)$ & $119.3(3)$ \\
\hline $\mathrm{C}(2)-\mathrm{C}(1)-\mathrm{P}(1)$ & $123.5(3)$ & $\mathrm{C}(14)-\mathrm{C}(13)-\mathrm{P}(1)$ & $119.1(3)$ \\
\hline $\mathrm{C}(6)-\mathrm{C}(1)-\mathrm{P}(1)$ & $117.2(2)$ & $\mathrm{C}(18)-\mathrm{C}(13)-\mathrm{P}(1)$ & $120.7(3)$ \\
\hline $\mathrm{C}(3)-\mathrm{C}(2)-\mathrm{C}(1)$ & $120.7(3)$ & $\mathrm{C}(13)-\mathrm{C}(14)-\mathrm{C}(15)$ & $121.4(3)$ \\
\hline $\mathrm{C}(3)-\mathrm{C}(2)-\mathrm{H}(2 \mathrm{~A})$ & 119.6 & $\mathrm{C}(13)-\mathrm{C}(14)-\mathrm{H}(14 \mathrm{~A})$ & 119.3 \\
\hline $\mathrm{C}(1)-\mathrm{C}(2)-\mathrm{H}(2 \mathrm{~A})$ & 119.6 & $\mathrm{C}(15)-\mathrm{C}(14)-\mathrm{H}(14 \mathrm{~A})$ & 119.3 \\
\hline $\mathrm{C}(4)-\mathrm{C}(3)-\mathrm{C}(2)$ & $119.8(3)$ & $\mathrm{C}(16)-\mathrm{C}(15)-\mathrm{C}(14)$ & $117.4(3)$ \\
\hline $\mathrm{C}(4)-\mathrm{C}(3)-\mathrm{H}(3 \mathrm{~A})$ & 120.1 & $\mathrm{C}(16)-\mathrm{C}(15)-\mathrm{C}(39)$ & $123.1(3)$ \\
\hline $\mathrm{C}(2)-\mathrm{C}(3)-\mathrm{H}(3 \mathrm{~A})$ & 120.1 & $\mathrm{C}(14)-\mathrm{C}(15)-\mathrm{C}(39)$ & $119.5(3)$ \\
\hline$C(5)-C(4)-C(3)$ & $120.0(3)$ & $\mathrm{C}(15)-\mathrm{C}(16)-\mathrm{C}(17)$ & $123.0(3)$ \\
\hline $\mathrm{C}(5)-\mathrm{C}(4)-\mathrm{H}(4 \mathrm{~A})$ & 120.0 & $\mathrm{C}(15)-\mathrm{C}(16)-\mathrm{H}(16 \mathrm{~A})$ & 118.5 \\
\hline $\mathrm{C}(3)-\mathrm{C}(4)-\mathrm{H}(4 \mathrm{~A})$ & 120.0 & $\mathrm{C}(17)-\mathrm{C}(16)-\mathrm{H}(16 \mathrm{~A})$ & 118.5 \\
\hline$C(4)-C(5)-C(6)$ & $121.1(3)$ & $\mathrm{C}(18)-\mathrm{C}(17)-\mathrm{C}(16)$ & $117.8(3)$ \\
\hline $\mathrm{C}(4)-\mathrm{C}(5)-\mathrm{H}(5 \mathrm{~A})$ & 119.5 & $C(18)-C(17)-C(43)$ & $122.9(3)$ \\
\hline $\mathrm{C}(6)-\mathrm{C}(5)-\mathrm{H}(5 \mathrm{~A})$ & 119.5 & $C(16)-C(17)-C(43)$ & $119.3(3)$ \\
\hline$C(5)-C(6)-C(1)$ & $119.1(3)$ & $\mathrm{C}(17)-\mathrm{C}(18)-\mathrm{C}(13)$ & $121.0(3)$ \\
\hline$C(5)-C(6)-P(2)$ & $124.4(3)$ & $\mathrm{C}(17)-\mathrm{C}(18)-\mathrm{H}(18 \mathrm{~A})$ & 119.5 \\
\hline$C(1)-C(6)-P(2)$ & $116.4(2)$ & $\mathrm{C}(13)-\mathrm{C}(18)-\mathrm{H}(18 \mathrm{~A})$ & 119.5 \\
\hline $\mathrm{C}(12)-\mathrm{C}(7)-\mathrm{C}(8)$ & $119.7(3)$ & $C(20)-C(19)-C(24)$ & $118.8(3)$ \\
\hline$C(12)-C(7)-P(1)$ & $125.4(3)$ & $\mathrm{C}(20)-\mathrm{C}(19)-\mathrm{P}(2)$ & $125.9(3)$ \\
\hline $\mathrm{C}(8)-\mathrm{C}(7)-\mathrm{P}(1)$ & $115.0(2)$ & $\mathrm{C}(24)-\mathrm{C}(19)-\mathrm{P}(2)$ & $115.3(3)$ \\
\hline$C(9)-C(8)-C(7)$ & $121.5(3)$ & $C(19)-C(20)-C(21)$ & $121.4(3)$ \\
\hline $\mathrm{C}(9)-\mathrm{C}(8)-\mathrm{H}(8 \mathrm{~A})$ & 119.2 & $\mathrm{C}(19)-\mathrm{C}(20)-\mathrm{H}(20 \mathrm{~A})$ & 119.3 \\
\hline $\mathrm{C}(7)-\mathrm{C}(8)-\mathrm{H}(8 \mathrm{~A})$ & 119.2 & $\mathrm{C}(21)-\mathrm{C}(20)-\mathrm{H}(20 \mathrm{~A})$ & 119.3 \\
\hline $\mathrm{C}(8)-\mathrm{C}(9)-\mathrm{C}(10)$ & $117.1(3)$ & $C(22)-C(21)-C(20)$ & $117.4(3)$ \\
\hline $\mathrm{C}(8)-\mathrm{C}(9)-\mathrm{C}(31)$ & $122.6(3)$ & $\mathrm{C}(22)-\mathrm{C}(21)-\mathrm{C}(51)$ & $121.3(3)$ \\
\hline $\mathrm{C}(10)-\mathrm{C}(9)-\mathrm{C}(31)$ & $120.3(3)$ & $\mathrm{C}(20)-\mathrm{C}(21)-\mathrm{C}(51)$ & $121.2(3)$ \\
\hline$C(11)-C(10)-C(9)$ & $122.9(3)$ & $C(21)-C(22)-C(23)$ & $123.2(3)$ \\
\hline $\mathrm{C}(11)-\mathrm{C}(10)-\mathrm{H}(10 \mathrm{~A})$ & 118.6 & $\mathrm{C}(21)-\mathrm{C}(22)-\mathrm{H}(22 \mathrm{~A})$ & 118.4 \\
\hline $\mathrm{C}(9)-\mathrm{C}(10)-\mathrm{H}(10 \mathrm{~A})$ & 118.6 & $\mathrm{C}(23)-\mathrm{C}(22)-\mathrm{H}(22 \mathrm{~A})$ & 118.4 \\
\hline $\mathrm{C}(10)-\mathrm{C}(11)-\mathrm{C}(12)$ & $117.7(3)$ & $\mathrm{C}(24)-\mathrm{C}(23)-\mathrm{C}(22)$ & $116.9(3)$ \\
\hline$C(10)-C(11)-C(35)$ & $122.2(3)$ & $C(24)-C(23)-C(47)$ & $122.6(3)$ \\
\hline$C(12)-C(11)-C(35)$ & $120.1(3)$ & $C(22)-C(23)-C(47)$ & $120.5(3)$ \\
\hline $\mathrm{C}(7)-\mathrm{C}(12)-\mathrm{C}(11)$ & $121.1(3)$ & $C(23)-C(24)-C(19)$ & $122.3(3)$ \\
\hline
\end{tabular}




\begin{tabular}{|c|c|}
\hline $\mathrm{C}(23)-\mathrm{C}(24)-\mathrm{H}(24 \mathrm{~A})$ & 118.9 \\
\hline $\mathrm{C}(19)-\mathrm{C}(24)-\mathrm{H}(24 \mathrm{~A})$ & 118.9 \\
\hline$C(26)-C(25)-C(30)$ & $119.2(3)$ \\
\hline $\mathrm{C}(26)-\mathrm{C}(25)-\mathrm{P}(2)$ & $123.2(3)$ \\
\hline $\mathrm{C}(30)-\mathrm{C}(25)-\mathrm{P}(2)$ & $117.6(2)$ \\
\hline$C(25)-C(26)-C(27)$ & $121.2(3)$ \\
\hline $\mathrm{C}(25)-\mathrm{C}(26)-\mathrm{H}(26 \mathrm{~A})$ & 119.4 \\
\hline $\mathrm{C}(27)-\mathrm{C}(26)-\mathrm{H}(26 \mathrm{~A})$ & 119.4 \\
\hline $\mathrm{C}(28)-\mathrm{C}(27)-\mathrm{C}(26)$ & $117.5(3)$ \\
\hline $\mathrm{C}(28)-\mathrm{C}(27)-\mathrm{C}(55)$ & $120.0(4)$ \\
\hline$C(26)-C(27)-C(55)$ & $122.4(4)$ \\
\hline $\mathrm{C}(28)-\mathrm{C}(27)-\mathrm{C}\left(55^{\prime}\right)$ & $123.2(5)$ \\
\hline$C(26)-C(27)-C\left(55^{\prime}\right)$ & $119.1(5)$ \\
\hline $\mathrm{C}(27)-\mathrm{C}(28)-\mathrm{C}(29)$ & $123.2(3)$ \\
\hline $\mathrm{C}(27)-\mathrm{C}(28)-\mathrm{H}(28 \mathrm{~A})$ & 118.4 \\
\hline $\mathrm{C}(29)-\mathrm{C}(28)-\mathrm{H}(28 \mathrm{~A})$ & 118.4 \\
\hline $\mathrm{C}(30)-\mathrm{C}(29)-\mathrm{C}(28)$ & $117.3(3)$ \\
\hline $\mathrm{C}(30)-\mathrm{C}(29)-\mathrm{C}(59)$ & $123.3(3)$ \\
\hline $\mathrm{C}(28)-\mathrm{C}(29)-\mathrm{C}(59)$ & $119.4(3)$ \\
\hline$C(29)-C(30)-C(25)$ & $121.5(3)$ \\
\hline $\mathrm{C}(29)-\mathrm{C}(30)-\mathrm{H}(30 \mathrm{~A})$ & 119.2 \\
\hline $\mathrm{C}(25)-\mathrm{C}(30)-\mathrm{H}(30 \mathrm{~A})$ & 119.2 \\
\hline$C(33)-C(31)-C(34)$ & $108.8(3)$ \\
\hline $\mathrm{C}(33)-\mathrm{C}(31)-\mathrm{C}(9)$ & $110.0(3)$ \\
\hline$C(34)-C(31)-C(9)$ & $112.3(3)$ \\
\hline$C(33)-C(31)-C(32)$ & $109.9(3)$ \\
\hline $\mathrm{C}(34)-\mathrm{C}(31)-\mathrm{C}(32)$ & $107.5(3)$ \\
\hline $\mathrm{C}(9)-\mathrm{C}(31)-\mathrm{C}(32)$ & $108.5(3)$ \\
\hline $\mathrm{C}(31)-\mathrm{C}(32)-\mathrm{H}(32 \mathrm{~A})$ & 109.5 \\
\hline $\mathrm{C}(31)-\mathrm{C}(32)-\mathrm{H}(32 \mathrm{~B})$ & 109.5 \\
\hline $\mathrm{H}(32 \mathrm{~A})-\mathrm{C}(32)-\mathrm{H}(32 \mathrm{~B})$ & 109.5 \\
\hline $\mathrm{C}(31)-\mathrm{C}(32)-\mathrm{H}(32 \mathrm{C})$ & 109.5 \\
\hline $\mathrm{H}(32 \mathrm{~A})-\mathrm{C}(32)-\mathrm{H}(32 \mathrm{C})$ & 109.5 \\
\hline $\mathrm{H}(32 \mathrm{~B})-\mathrm{C}(32)-\mathrm{H}(32 \mathrm{C})$ & 109.5 \\
\hline $\mathrm{C}(31)-\mathrm{C}(33)-\mathrm{H}(33 \mathrm{~A})$ & 109.5 \\
\hline $\mathrm{C}(31)-\mathrm{C}(33)-\mathrm{H}(33 \mathrm{~B})$ & 109.5 \\
\hline
\end{tabular}

\begin{tabular}{|c|c|}
\hline $\mathrm{H}(33 \mathrm{~A})-\mathrm{C}(33)-\mathrm{H}(33 \mathrm{~B})$ & 109.5 \\
\hline $\mathrm{C}(31)-\mathrm{C}(33)-\mathrm{H}(33 \mathrm{C})$ & 109.5 \\
\hline$H(33 A)-C(33)-H(33 C)$ & 109.5 \\
\hline $\mathrm{H}(33 \mathrm{~B})-\mathrm{C}(33)-\mathrm{H}(33 \mathrm{C})$ & 109.5 \\
\hline $\mathrm{C}(31)-\mathrm{C}(34)-\mathrm{H}(34 \mathrm{~A})$ & 109.5 \\
\hline $\mathrm{C}(31)-\mathrm{C}(34)-\mathrm{H}(34 \mathrm{~B})$ & 109.5 \\
\hline $\mathrm{H}(34 \mathrm{~A})-\mathrm{C}(34)-\mathrm{H}(34 \mathrm{~B})$ & 109.5 \\
\hline $\mathrm{C}(31)-\mathrm{C}(34)-\mathrm{H}(34 \mathrm{C})$ & 109.5 \\
\hline $\mathrm{H}(34 \mathrm{~A})-\mathrm{C}(34)-\mathrm{H}(34 \mathrm{C})$ & 109.5 \\
\hline $\mathrm{H}(34 \mathrm{~B})-\mathrm{C}(34)-\mathrm{H}(34 \mathrm{C})$ & 109.5 \\
\hline $\mathrm{C}(37)-\mathrm{C}(35)-\mathrm{C}(38)$ & $108.3(3)$ \\
\hline $\mathrm{C}(37)-\mathrm{C}(35)-\mathrm{C}(11)$ & $112.6(3)$ \\
\hline $\mathrm{C}(38)-\mathrm{C}(35)-\mathrm{C}(11)$ & 109.1(3) \\
\hline $\mathrm{C}(37)-\mathrm{C}(35)-\mathrm{C}(36)$ & $108.7(3)$ \\
\hline $\mathrm{C}(38)-\mathrm{C}(35)-\mathrm{C}(36)$ & 109.1(3) \\
\hline $\mathrm{C}(11)-\mathrm{C}(35)-\mathrm{C}(36)$ & $109.0(3)$ \\
\hline $\mathrm{C}(35)-\mathrm{C}(36)-\mathrm{H}(36 \mathrm{~A})$ & 109.5 \\
\hline $\mathrm{C}(35)-\mathrm{C}(36)-\mathrm{H}(36 \mathrm{~B})$ & 109.5 \\
\hline $\mathrm{H}(36 \mathrm{~A})-\mathrm{C}(36)-\mathrm{H}(36 \mathrm{~B})$ & 109.5 \\
\hline $\mathrm{C}(35)-\mathrm{C}(36)-\mathrm{H}(36 \mathrm{C})$ & 109.5 \\
\hline $\mathrm{H}(36 \mathrm{~A})-\mathrm{C}(36)-\mathrm{H}(36 \mathrm{C})$ & 109.5 \\
\hline $\mathrm{H}(36 \mathrm{~B})-\mathrm{C}(36)-\mathrm{H}(36 \mathrm{C})$ & 109.5 \\
\hline $\mathrm{C}(35)-\mathrm{C}(37)-\mathrm{H}(37 \mathrm{~A})$ & 109.5 \\
\hline $\mathrm{C}(35)-\mathrm{C}(37)-\mathrm{H}(37 \mathrm{~B})$ & 109.5 \\
\hline $\mathrm{H}(37 \mathrm{~A})-\mathrm{C}(37)-\mathrm{H}(37 \mathrm{~B})$ & 109.5 \\
\hline $\mathrm{C}(35)-\mathrm{C}(37)-\mathrm{H}(37 \mathrm{C})$ & 109.5 \\
\hline $\mathrm{H}(37 \mathrm{~A})-\mathrm{C}(37)-\mathrm{H}(37 \mathrm{C})$ & 109.5 \\
\hline $\mathrm{H}(37 \mathrm{~B})-\mathrm{C}(37)-\mathrm{H}(37 \mathrm{C})$ & 109.5 \\
\hline $\mathrm{C}(35)-\mathrm{C}(38)-\mathrm{H}(38 \mathrm{~A})$ & 109.5 \\
\hline $\mathrm{C}(35)-\mathrm{C}(38)-\mathrm{H}(38 \mathrm{~B})$ & 109.5 \\
\hline $\mathrm{H}(38 \mathrm{~A})-\mathrm{C}(38)-\mathrm{H}(38 \mathrm{~B})$ & 109.5 \\
\hline $\mathrm{C}(35)-\mathrm{C}(38)-\mathrm{H}(38 \mathrm{C})$ & 109.5 \\
\hline $\mathrm{H}(38 \mathrm{~A})-\mathrm{C}(38)-\mathrm{H}(38 \mathrm{C})$ & 109.5 \\
\hline $\mathrm{H}(38 \mathrm{~B})-\mathrm{C}(38)-\mathrm{H}(38 \mathrm{C})$ & 109.5 \\
\hline $\mathrm{C}(42)-\mathrm{C}(39)-\mathrm{C}(40)$ & 109.1(3) \\
\hline $\mathrm{C}(42)-\mathrm{C}(39)-\mathrm{C}(41)$ & $108.6(3)$ \\
\hline
\end{tabular}




\begin{tabular}{|c|c|}
\hline$C(40)-C(39)-C(41)$ & $109.2(3)$ \\
\hline$C(42)-C(39)-C(15)$ & $112.1(3)$ \\
\hline$C(40)-C(39)-C(15)$ & $108.8(3)$ \\
\hline$C(41)-C(39)-C(15)$ & $109.0(3)$ \\
\hline $\mathrm{C}(39)-\mathrm{C}(40)-\mathrm{H}(40 \mathrm{~A})$ & 109.5 \\
\hline $\mathrm{C}(39)-\mathrm{C}(40)-\mathrm{H}(40 \mathrm{~B})$ & 109.5 \\
\hline $\mathrm{H}(40 \mathrm{~A})-\mathrm{C}(40)-\mathrm{H}(40 \mathrm{~B})$ & 109.5 \\
\hline $\mathrm{C}(39)-\mathrm{C}(40)-\mathrm{H}(40 \mathrm{C})$ & 109.5 \\
\hline $\mathrm{H}(40 \mathrm{~A})-\mathrm{C}(40)-\mathrm{H}(40 \mathrm{C})$ & 109.5 \\
\hline $\mathrm{H}(40 \mathrm{~B})-\mathrm{C}(40)-\mathrm{H}(40 \mathrm{C})$ & 109.5 \\
\hline $\mathrm{C}(39)-\mathrm{C}(41)-\mathrm{H}(41 \mathrm{~A})$ & 109.5 \\
\hline $\mathrm{C}(39)-\mathrm{C}(41)-\mathrm{H}(41 \mathrm{~B})$ & 109.5 \\
\hline $\mathrm{H}(41 \mathrm{~A})-\mathrm{C}(41)-\mathrm{H}(41 \mathrm{~B})$ & 109.5 \\
\hline C(39)-C(41)-H(41C) & 109.5 \\
\hline $\mathrm{H}(41 \mathrm{~A})-\mathrm{C}(41)-\mathrm{H}(41 \mathrm{C})$ & 109.5 \\
\hline $\mathrm{H}(41 \mathrm{~B})-\mathrm{C}(41)-\mathrm{H}(41 \mathrm{C})$ & 109.5 \\
\hline $\mathrm{C}(39)-\mathrm{C}(42)-\mathrm{H}(42 \mathrm{~A})$ & 109.5 \\
\hline $\mathrm{C}(39)-\mathrm{C}(42)-\mathrm{H}(42 \mathrm{~B})$ & 109.5 \\
\hline $\mathrm{H}(42 \mathrm{~A})-\mathrm{C}(42)-\mathrm{H}(42 \mathrm{~B})$ & 109.5 \\
\hline $\mathrm{C}(39)-\mathrm{C}(42)-\mathrm{H}(42 \mathrm{C})$ & 109.5 \\
\hline $\mathrm{H}(42 \mathrm{~A})-\mathrm{C}(42)-\mathrm{H}(42 \mathrm{C})$ & 109.5 \\
\hline $\mathrm{H}(42 \mathrm{~B})-\mathrm{C}(42)-\mathrm{H}(42 \mathrm{C})$ & 109.5 \\
\hline$C(45)-C(43)-C(17)$ & $111.6(3)$ \\
\hline$C(45)-C(43)-C(44)$ & $108.7(3)$ \\
\hline$C(17)-C(43)-C(44)$ & $109.0(3)$ \\
\hline$C(45)-C(43)-C(46)$ & $108.8(3)$ \\
\hline$C(17)-C(43)-C(46)$ & $109.1(3)$ \\
\hline $\mathrm{C}(44)-\mathrm{C}(43)-\mathrm{C}(46)$ & $109.6(3)$ \\
\hline $\mathrm{C}(43)-\mathrm{C}(44)-\mathrm{H}(44 \mathrm{~A})$ & 109.5 \\
\hline $\mathrm{C}(43)-\mathrm{C}(44)-\mathrm{H}(44 \mathrm{~B})$ & 109.5 \\
\hline $\mathrm{H}(44 \mathrm{~A})-\mathrm{C}(44)-\mathrm{H}(44 \mathrm{~B})$ & 109.5 \\
\hline $\mathrm{C}(43)-\mathrm{C}(44)-\mathrm{H}(44 \mathrm{C})$ & 109.5 \\
\hline $\mathrm{H}(44 \mathrm{~A})-\mathrm{C}(44)-\mathrm{H}(44 \mathrm{C})$ & 109.5 \\
\hline $\mathrm{H}(44 \mathrm{~B})-\mathrm{C}(44)-\mathrm{H}(44 \mathrm{C})$ & 109.5 \\
\hline $\mathrm{C}(43)-\mathrm{C}(45)-\mathrm{H}(45 \mathrm{~A})$ & 109.5 \\
\hline $\mathrm{C}(43)-\mathrm{C}(45)-\mathrm{H}(45 \mathrm{~B})$ & 109.5 \\
\hline
\end{tabular}

\begin{tabular}{|c|c|}
\hline $\mathrm{H}(45 \mathrm{~A})-\mathrm{C}(45)-\mathrm{H}(45 \mathrm{~B})$ & 109.5 \\
\hline $\mathrm{C}(43)-\mathrm{C}(45)-\mathrm{H}(45 \mathrm{C})$ & 109.5 \\
\hline $\mathrm{H}(45 \mathrm{~A})-\mathrm{C}(45)-\mathrm{H}(45 \mathrm{C})$ & 109.5 \\
\hline $\mathrm{H}(45 \mathrm{~B})-\mathrm{C}(45)-\mathrm{H}(45 \mathrm{C})$ & 109.5 \\
\hline $\mathrm{C}(43)-\mathrm{C}(46)-\mathrm{H}(46 \mathrm{~A})$ & 109.5 \\
\hline $\mathrm{C}(43)-\mathrm{C}(46)-\mathrm{H}(46 \mathrm{~B})$ & 109.5 \\
\hline $\mathrm{H}(46 \mathrm{~A})-\mathrm{C}(46)-\mathrm{H}(46 \mathrm{~B})$ & 109.5 \\
\hline $\mathrm{C}(43)-\mathrm{C}(46)-\mathrm{H}(46 \mathrm{C})$ & 109.5 \\
\hline $\mathrm{H}(46 \mathrm{~A})-\mathrm{C}(46)-\mathrm{H}(46 \mathrm{C})$ & 109.5 \\
\hline $\mathrm{H}(46 \mathrm{~B})-\mathrm{C}(46)-\mathrm{H}(46 \mathrm{C})$ & 109.5 \\
\hline $\mathrm{C}(50)-\mathrm{C}(47)-\mathrm{C}(23)$ & $111.8(3)$ \\
\hline $\mathrm{C}(50)-\mathrm{C}(47)-\mathrm{C}(48)$ & $107.9(3)$ \\
\hline $\mathrm{C}(23)-\mathrm{C}(47)-\mathrm{C}(48)$ & $111.0(3)$ \\
\hline $\mathrm{C}(50)-\mathrm{C}(47)-\mathrm{C}(49)$ & $109.2(3)$ \\
\hline $\mathrm{C}(23)-\mathrm{C}(47)-\mathrm{C}(49)$ & $107.8(3)$ \\
\hline $\mathrm{C}(48)-\mathrm{C}(47)-\mathrm{C}(49)$ & 109.1(3) \\
\hline $\mathrm{C}(47)-\mathrm{C}(48)-\mathrm{H}(48 \mathrm{~A})$ & 109.5 \\
\hline $\mathrm{C}(47)-\mathrm{C}(48)-\mathrm{H}(48 \mathrm{~B})$ & 109.5 \\
\hline $\mathrm{H}(48 \mathrm{~A})-\mathrm{C}(48)-\mathrm{H}(48 \mathrm{~B})$ & 109.5 \\
\hline $\mathrm{C}(47)-\mathrm{C}(48)-\mathrm{H}(48 \mathrm{C})$ & 109.5 \\
\hline $\mathrm{H}(48 \mathrm{~A})-\mathrm{C}(48)-\mathrm{H}(48 \mathrm{C})$ & 109.5 \\
\hline $\mathrm{H}(48 \mathrm{~B})-\mathrm{C}(48)-\mathrm{H}(48 \mathrm{C})$ & 109.5 \\
\hline $\mathrm{C}(47)-\mathrm{C}(49)-\mathrm{H}(49 \mathrm{~A})$ & 109.5 \\
\hline $\mathrm{C}(47)-\mathrm{C}(49)-\mathrm{H}(49 \mathrm{~B})$ & 109.5 \\
\hline $\mathrm{H}(49 \mathrm{~A})-\mathrm{C}(49)-\mathrm{H}(49 \mathrm{~B})$ & 109.5 \\
\hline $\mathrm{C}(47)-\mathrm{C}(49)-\mathrm{H}(49 \mathrm{C})$ & 109.5 \\
\hline $\mathrm{H}(49 \mathrm{~A})-\mathrm{C}(49)-\mathrm{H}(49 \mathrm{C})$ & 109.5 \\
\hline $\mathrm{H}(49 \mathrm{~B})-\mathrm{C}(49)-\mathrm{H}(49 \mathrm{C})$ & 109.5 \\
\hline $\mathrm{C}(47)-\mathrm{C}(50)-\mathrm{H}(50 \mathrm{~A})$ & 109.5 \\
\hline $\mathrm{C}(47)-\mathrm{C}(50)-\mathrm{H}(50 \mathrm{~B})$ & 109.5 \\
\hline $\mathrm{H}(50 \mathrm{~A})-\mathrm{C}(50)-\mathrm{H}(50 \mathrm{~B})$ & 109.5 \\
\hline $\mathrm{C}(47)-\mathrm{C}(50)-\mathrm{H}(50 \mathrm{C})$ & 109.5 \\
\hline $\mathrm{H}(50 \mathrm{~A})-\mathrm{C}(50)-\mathrm{H}(50 \mathrm{C})$ & 109.5 \\
\hline $\mathrm{H}(50 \mathrm{~B})-\mathrm{C}(50)-\mathrm{H}(50 \mathrm{C})$ & 109.5 \\
\hline $\mathrm{C}(52)-\mathrm{C}(51)-\mathrm{C}(21)$ & $111.0(3)$ \\
\hline $\mathrm{C}(52)-\mathrm{C}(51)-\mathrm{C}(54)$ & $109.6(3)$ \\
\hline
\end{tabular}




\begin{tabular}{|c|c|c|c|}
\hline$C(21)-C(51)-C(54)$ & $108.0(3)$ & $\mathrm{H}(57 \mathrm{~A})-\mathrm{C}(57)-\mathrm{H}(57 \mathrm{~B})$ & 109.5 \\
\hline$C(52)-C(51)-C(53)$ & $107.5(3)$ & $\mathrm{C}(55)-\mathrm{C}(57)-\mathrm{H}(57 \mathrm{C})$ & 109.5 \\
\hline$C(21)-C(51)-C(53)$ & $112.6(3)$ & $\mathrm{H}(57 \mathrm{~A})-\mathrm{C}(57)-\mathrm{H}(57 \mathrm{C})$ & 109.5 \\
\hline$C(54)-C(51)-C(53)$ & $108.1(3)$ & $\mathrm{H}(57 \mathrm{~B})-\mathrm{C}(57)-\mathrm{H}(57 \mathrm{C})$ & 109.5 \\
\hline $\mathrm{C}(51)-\mathrm{C}(52)-\mathrm{H}(52 \mathrm{~A})$ & 109.5 & $\mathrm{C}(55)-\mathrm{C}(58)-\mathrm{H}(58 \mathrm{~A})$ & 109.5 \\
\hline $\mathrm{C}(51)-\mathrm{C}(52)-\mathrm{H}(52 \mathrm{~B})$ & 109.5 & $\mathrm{C}(55)-\mathrm{C}(58)-\mathrm{H}(58 \mathrm{~B})$ & 109.5 \\
\hline $\mathrm{H}(52 \mathrm{~A})-\mathrm{C}(52)-\mathrm{H}(52 \mathrm{~B})$ & 109.5 & $\mathrm{H}(58 \mathrm{~A})-\mathrm{C}(58)-\mathrm{H}(58 \mathrm{~B})$ & 109.5 \\
\hline $\mathrm{C}(51)-\mathrm{C}(52)-\mathrm{H}(52 \mathrm{C})$ & 109.5 & $\mathrm{C}(55)-\mathrm{C}(58)-\mathrm{H}(58 \mathrm{C})$ & 109.5 \\
\hline $\mathrm{H}(52 \mathrm{~A})-\mathrm{C}(52)-\mathrm{H}(52 \mathrm{C})$ & 109.5 & $\mathrm{H}(58 \mathrm{~A})-\mathrm{C}(58)-\mathrm{H}(58 \mathrm{C})$ & 109.5 \\
\hline $\mathrm{H}(52 \mathrm{~B})-\mathrm{C}(52)-\mathrm{H}(52 \mathrm{C})$ & 109.5 & $\mathrm{H}(58 \mathrm{~B})-\mathrm{C}(58)-\mathrm{H}(58 \mathrm{C})$ & 109.5 \\
\hline $\mathrm{C}(51)-\mathrm{C}(53)-\mathrm{H}(53 \mathrm{~A})$ & 109.5 & $\mathrm{C}\left(56^{\prime}\right)-\mathrm{C}\left(55^{\prime}\right)-\mathrm{C}(27)$ & $111.9(6)$ \\
\hline $\mathrm{C}(51)-\mathrm{C}(53)-\mathrm{H}(53 \mathrm{~B})$ & 109.5 & $\mathrm{C}\left(56^{\prime}\right)-\mathrm{C}\left(55^{\prime}\right)-\mathrm{C}\left(58^{\prime}\right)$ & $108.0(7)$ \\
\hline $\mathrm{H}(53 \mathrm{~A})-\mathrm{C}(53)-\mathrm{H}(53 \mathrm{~B})$ & 109.5 & $\mathrm{C}(27)-\mathrm{C}\left(55^{\prime}\right)-\mathrm{C}\left(58^{\prime}\right)$ & $112.1(7)$ \\
\hline $\mathrm{C}(51)-\mathrm{C}(53)-\mathrm{H}(53 \mathrm{C})$ & 109.5 & $\mathrm{C}\left(56^{\prime}\right)-\mathrm{C}\left(55^{\prime}\right)-\mathrm{C}\left(57^{\prime}\right)$ & $108.8(7)$ \\
\hline $\mathrm{H}(53 \mathrm{~A})-\mathrm{C}(53)-\mathrm{H}(53 \mathrm{C})$ & 109.5 & $\mathrm{C}(27)-\mathrm{C}\left(55^{\prime}\right)-\mathrm{C}\left(57^{\prime}\right)$ & $108.1(7)$ \\
\hline $\mathrm{H}(53 \mathrm{~B})-\mathrm{C}(53)-\mathrm{H}(53 \mathrm{C})$ & 109.5 & $\mathrm{C}\left(58^{\prime}\right)-\mathrm{C}\left(55^{\prime}\right)-\mathrm{C}\left(57^{\prime}\right)$ & $107.7(7)$ \\
\hline $\mathrm{C}(51)-\mathrm{C}(54)-\mathrm{H}(54 \mathrm{~A})$ & 109.5 & $\mathrm{C}\left(55^{\prime}\right)-\mathrm{C}\left(56^{\prime}\right)-\mathrm{H}(56 \mathrm{D})$ & 109.5 \\
\hline $\mathrm{C}(51)-\mathrm{C}(54)-\mathrm{H}(54 \mathrm{~B})$ & 109.5 & $\mathrm{C}\left(55^{\prime}\right)-\mathrm{C}\left(56^{\prime}\right)-\mathrm{H}(56 \mathrm{E})$ & 109.5 \\
\hline $\mathrm{H}(54 \mathrm{~A})-\mathrm{C}(54)-\mathrm{H}(54 \mathrm{~B})$ & 109.5 & $\mathrm{H}(56 \mathrm{D})-\mathrm{C}\left(56^{\prime}\right)-\mathrm{H}(56 \mathrm{E})$ & 109.5 \\
\hline $\mathrm{C}(51)-\mathrm{C}(54)-\mathrm{H}(54 \mathrm{C})$ & 109.5 & $\mathrm{C}\left(55^{\prime}\right)-\mathrm{C}\left(56^{\prime}\right)-\mathrm{H}(56 \mathrm{~F})$ & 109.5 \\
\hline $\mathrm{H}(54 \mathrm{~A})-\mathrm{C}(54)-\mathrm{H}(54 \mathrm{C})$ & 109.5 & $\mathrm{H}(56 \mathrm{D})-\mathrm{C}\left(56^{\prime}\right)-\mathrm{H}(56 \mathrm{~F})$ & 109.5 \\
\hline $\mathrm{H}(54 \mathrm{~B})-\mathrm{C}(54)-\mathrm{H}(54 \mathrm{C})$ & 109.5 & $\mathrm{H}(56 \mathrm{E})-\mathrm{C}\left(56^{\prime}\right)-\mathrm{H}(56 \mathrm{~F})$ & 109.5 \\
\hline $\mathrm{C}(57)-\mathrm{C}(55)-\mathrm{C}(58)$ & $110.1(6)$ & $\mathrm{C}\left(55^{\prime}\right)-\mathrm{C}\left(57^{\prime}\right)-\mathrm{H}(57 \mathrm{D})$ & 109.5 \\
\hline$C(57)-C(55)-C(27)$ & $108.4(5)$ & $\mathrm{C}\left(55^{\prime}\right)-\mathrm{C}\left(57^{\prime}\right)-\mathrm{H}(57 \mathrm{E})$ & 109.5 \\
\hline $\mathrm{C}(58)-\mathrm{C}(55)-\mathrm{C}(27)$ & $110.9(5)$ & $\mathrm{H}(57 \mathrm{D})-\mathrm{C}\left(57^{\prime}\right)-\mathrm{H}(57 \mathrm{E})$ & 109.5 \\
\hline$C(57)-C(55)-C(56)$ & $108.9(5)$ & $\mathrm{C}\left(55^{\prime}\right)-\mathrm{C}\left(57^{\prime}\right)-\mathrm{H}(57 \mathrm{~F})$ & 109.5 \\
\hline$C(58)-C(55)-C(56)$ & $107.9(6)$ & $\mathrm{H}(57 \mathrm{D})-\mathrm{C}\left(57^{\prime}\right)-\mathrm{H}(57 \mathrm{~F})$ & 109.5 \\
\hline$C(27)-C(55)-C(56)$ & $110.6(5)$ & $\mathrm{H}(57 \mathrm{E})-\mathrm{C}\left(57^{\prime}\right)-\mathrm{H}(57 \mathrm{~F})$ & 109.5 \\
\hline $\mathrm{C}(55)-\mathrm{C}(56)-\mathrm{H}(56 \mathrm{~A})$ & 109.5 & $\mathrm{C}\left(55^{\prime}\right)-\mathrm{C}\left(58^{\prime}\right)-\mathrm{H}(58 \mathrm{D})$ & 109.5 \\
\hline $\mathrm{C}(55)-\mathrm{C}(56)-\mathrm{H}(56 \mathrm{~B})$ & 109.5 & $\mathrm{C}\left(55^{\prime}\right)-\mathrm{C}\left(58^{\prime}\right)-\mathrm{H}(58 \mathrm{E})$ & 109.5 \\
\hline $\mathrm{H}(56 \mathrm{~A})-\mathrm{C}(56)-\mathrm{H}(56 \mathrm{~B})$ & 109.5 & $\mathrm{H}(58 \mathrm{D})-\mathrm{C}\left(58^{\prime}\right)-\mathrm{H}(58 \mathrm{E})$ & 109.5 \\
\hline $\mathrm{C}(55)-\mathrm{C}(56)-\mathrm{H}(56 \mathrm{C})$ & 109.5 & $\mathrm{C}\left(55^{\prime}\right)-\mathrm{C}\left(58^{\prime}\right)-\mathrm{H}(58 \mathrm{~F})$ & 109.5 \\
\hline $\mathrm{H}(56 \mathrm{~A})-\mathrm{C}(56)-\mathrm{H}(56 \mathrm{C})$ & 109.5 & $\mathrm{H}(58 \mathrm{D})-\mathrm{C}\left(58^{\prime}\right)-\mathrm{H}(58 \mathrm{~F})$ & 109.5 \\
\hline $\mathrm{H}(56 \mathrm{~B})-\mathrm{C}(56)-\mathrm{H}(56 \mathrm{C})$ & 109.5 & $\mathrm{H}(58 \mathrm{E})-\mathrm{C}\left(58^{\prime}\right)-\mathrm{H}(58 \mathrm{~F})$ & 109.5 \\
\hline $\mathrm{C}(55)-\mathrm{C}(57)-\mathrm{H}(57 \mathrm{~A})$ & 109.5 & $\mathrm{C}(29)-\mathrm{C}(59)-\mathrm{C}(62)$ & $109.7(3)$ \\
\hline $\mathrm{C}(55)-\mathrm{C}(57)-\mathrm{H}(57 \mathrm{~B})$ & 109.5 & $\mathrm{C}(29)-\mathrm{C}(59)-\mathrm{C}(60)$ & $112.3(3)$ \\
\hline
\end{tabular}




\begin{tabular}{|c|c|c|c|}
\hline$C(62)-C(59)-C(60)$ & $107.9(3)$ & $\mathrm{H}(63 \mathrm{~A})-\mathrm{C}(63)-\mathrm{H}(63 \mathrm{~B})$ & 109.5 \\
\hline$C(29)-C(59)-C(61)$ & $109.5(3)$ & $\mathrm{C}(64)-\mathrm{C}(63)-\mathrm{H}(63 \mathrm{C})$ & 109.5 \\
\hline$C(62)-C(59)-C(61)$ & 109.1(3) & $H(63 A)-C(63)-H(63 C)$ & 109.5 \\
\hline $\mathrm{C}(60)-\mathrm{C}(59)-\mathrm{C}(61)$ & 108.3(3) & H(63B)-C(63)-H(63C) & 109.5 \\
\hline $\mathrm{C}(59)-\mathrm{C}(60)-\mathrm{H}(60 \mathrm{~A})$ & 109.5 & $\mathrm{O}(1)-\mathrm{C}(64)-\mathrm{C}(63)$ & $105.6(16)$ \\
\hline $\mathrm{C}(59)-\mathrm{C}(60)-\mathrm{H}(60 \mathrm{~B})$ & 109.5 & $\mathrm{O}(1)-\mathrm{C}(64)-\mathrm{H}(64 \mathrm{~A})$ & 110.6 \\
\hline $\mathrm{H}(60 \mathrm{~A})-\mathrm{C}(60)-\mathrm{H}(60 \mathrm{~B})$ & 109.5 & $\mathrm{C}(63)-\mathrm{C}(64)-\mathrm{H}(64 \mathrm{~A})$ & 110.6 \\
\hline $\mathrm{C}(59)-\mathrm{C}(60)-\mathrm{H}(60 \mathrm{C})$ & 109.5 & $\mathrm{O}(1)-\mathrm{C}(64)-\mathrm{H}(64 \mathrm{~B})$ & 110.6 \\
\hline$H(60 A)-C(60)-H(60 C)$ & 109.5 & $\mathrm{C}(63)-\mathrm{C}(64)-\mathrm{H}(64 \mathrm{~B})$ & 110.6 \\
\hline$H(60 B)-C(60)-H(60 C)$ & 109.5 & $H(64 A)-C(64)-H(64 B)$ & 108.8 \\
\hline $\mathrm{C}(59)-\mathrm{C}(61)-\mathrm{H}(61 \mathrm{~A})$ & 109.5 & $\mathrm{C}(64)-\mathrm{O}(1)-\mathrm{C}(65)$ & $108(2)$ \\
\hline $\mathrm{C}(59)-\mathrm{C}(61)-\mathrm{H}(61 \mathrm{~B})$ & 109.5 & $\mathrm{O}(1)-\mathrm{C}(65)-\mathrm{C}(66)$ & $103.5(14)$ \\
\hline $\mathrm{H}(61 \mathrm{~A})-\mathrm{C}(61)-\mathrm{H}(61 \mathrm{~B})$ & 109.5 & $\mathrm{O}(1)-\mathrm{C}(65)-\mathrm{H}(65 \mathrm{~A})$ & 111.1 \\
\hline $\mathrm{C}(59)-\mathrm{C}(61)-\mathrm{H}(61 \mathrm{C})$ & 109.5 & $\mathrm{C}(66)-\mathrm{C}(65)-\mathrm{H}(65 \mathrm{~A})$ & 111.1 \\
\hline$H(61 A)-C(61)-H(61 C)$ & 109.5 & $\mathrm{O}(1)-\mathrm{C}(65)-\mathrm{H}(65 \mathrm{~B})$ & 111.1 \\
\hline $\mathrm{H}(61 \mathrm{~B})-\mathrm{C}(61)-\mathrm{H}(61 \mathrm{C})$ & 109.5 & $\mathrm{C}(66)-\mathrm{C}(65)-\mathrm{H}(65 \mathrm{~B})$ & 111.1 \\
\hline $\mathrm{C}(59)-\mathrm{C}(62)-\mathrm{H}(62 \mathrm{~A})$ & 109.5 & $H(65 A)-C(65)-H(65 B)$ & 109.0 \\
\hline $\mathrm{C}(59)-\mathrm{C}(62)-\mathrm{H}(62 \mathrm{~B})$ & 109.5 & $\mathrm{C}(65)-\mathrm{C}(66)-\mathrm{H}(66 \mathrm{~A})$ & 109.5 \\
\hline $\mathrm{H}(62 \mathrm{~A})-\mathrm{C}(62)-\mathrm{H}(62 \mathrm{~B})$ & 109.5 & $\mathrm{C}(65)-\mathrm{C}(66)-\mathrm{H}(66 \mathrm{~B})$ & 109.5 \\
\hline $\mathrm{C}(59)-\mathrm{C}(62)-\mathrm{H}(62 \mathrm{C})$ & 109.5 & $\mathrm{H}(66 \mathrm{~A})-\mathrm{C}(66)-\mathrm{H}(66 \mathrm{~B})$ & 109.5 \\
\hline $\mathrm{H}(62 \mathrm{~A})-\mathrm{C}(62)-\mathrm{H}(62 \mathrm{C})$ & 109.5 & $\mathrm{C}(65)-\mathrm{C}(66)-\mathrm{H}(66 \mathrm{C})$ & 109.5 \\
\hline $\mathrm{H}(62 \mathrm{~B})-\mathrm{C}(62)-\mathrm{H}(62 \mathrm{C})$ & 109.5 & $\mathrm{H}(66 \mathrm{~A})-\mathrm{C}(66)-\mathrm{H}(66 \mathrm{C})$ & 109.5 \\
\hline $\mathrm{C}(64)-\mathrm{C}(63)-\mathrm{H}(63 \mathrm{~A})$ & 109.5 & $\mathrm{H}(66 \mathrm{~B})-\mathrm{C}(66)-\mathrm{H}(66 \mathrm{C})$ & 109.5 \\
\hline $\mathrm{C}(64)-\mathrm{C}(63)-\mathrm{H}(63 \mathrm{~B})$ & 109.5 & & \\
\hline
\end{tabular}

Symmetry transformations used to generate equivalent atoms:

$\# 1-\mathrm{x}+1 / 2, \mathrm{y},-\mathrm{z}+1 / 2$ 
Table S28. Anisotropic displacement parameters $\left(\AA^{2} \times 10^{3}\right)$ for neijk39. The anisotropic displacement factor exponent takes the form: $-2 \pi^{2}\left[h^{2} a^{* 2} U_{11}+\ldots+2 h k a^{*} b^{*} U_{12}\right]$

\begin{tabular}{|c|c|c|c|c|c|c|}
\hline & $\mathrm{U}_{11}$ & $\mathrm{U}_{22}$ & $\mathrm{U}_{33}$ & $\mathrm{U}_{23}$ & $\mathrm{U}_{13}$ & $\mathrm{U}_{12}$ \\
\hline $\mathrm{Fe} 1$ & $18(1)$ & $18(1)$ & $15(1)$ & $1(1)$ & $3(1)$ & 1(1) \\
\hline $\mathrm{Br} 1$ & $40(1)$ & $18(1)$ & $28(1)$ & 0 & $18(1)$ & 0 \\
\hline $\mathrm{Br} 2$ & $22(1)$ & $18(1)$ & $20(1)$ & 0 & $7(1)$ & 0 \\
\hline P1 & $19(1)$ & $17(1)$ & $14(1)$ & $0(1)$ & $2(1)$ & $0(1)$ \\
\hline $\mathrm{P} 2$ & $17(1)$ & $17(1)$ & $14(1)$ & $0(1)$ & $1(1)$ & $0(1)$ \\
\hline $\mathrm{C} 1$ & $21(2)$ & $12(2)$ & $19(2)$ & $2(1)$ & $4(2)$ & $-2(2)$ \\
\hline $\mathrm{C} 2$ & $22(2)$ & $20(2)$ & $19(2)$ & $1(1)$ & $1(2)$ & $2(2)$ \\
\hline $\mathrm{C} 3$ & $17(2)$ & $20(2)$ & $32(2)$ & $1(2)$ & $4(2)$ & $-2(2)$ \\
\hline $\mathrm{C} 4$ & $28(2)$ & $21(2)$ & $23(2)$ & $1(2)$ & $9(2)$ & $-1(2)$ \\
\hline $\mathrm{C} 5$ & $24(2)$ & $21(2)$ & $18(2)$ & $1(1)$ & $6(2)$ & $2(2)$ \\
\hline C6 & $21(2)$ & $13(2)$ & $21(2)$ & $-2(1)$ & $6(2)$ & $-2(1)$ \\
\hline C7 & $17(2)$ & $20(2)$ & $15(2)$ & $1(1)$ & $4(2)$ & $-1(2)$ \\
\hline $\mathrm{C} 8$ & $18(2)$ & $21(2)$ & $14(2)$ & $2(1)$ & $0(2)$ & $0(2)$ \\
\hline C9 & $22(2)$ & $19(2)$ & $16(2)$ & $2(1)$ & $5(2)$ & $0(2)$ \\
\hline $\mathrm{C} 10$ & $21(2)$ & $19(2)$ & $19(2)$ & $2(1)$ & $5(2)$ & $2(2)$ \\
\hline $\mathrm{C} 11$ & $18(2)$ & $22(2)$ & $12(2)$ & $1(1)$ & $3(1)$ & $1(2)$ \\
\hline $\mathrm{C} 12$ & $19(2)$ & $17(2)$ & $14(2)$ & $-1(1)$ & $2(2)$ & $-5(2)$ \\
\hline $\mathrm{C} 13$ & $15(2)$ & $18(2)$ & $18(2)$ & $-1(1)$ & $1(2)$ & $1(2)$ \\
\hline C14 & $19(2)$ & $16(2)$ & $21(2)$ & $1(1)$ & $1(2)$ & $-1(2)$ \\
\hline C15 & $20(2)$ & $21(2)$ & $16(2)$ & $-3(1)$ & $1(2)$ & $2(2)$ \\
\hline C16 & $21(2)$ & $23(2)$ & $16(2)$ & $-4(1)$ & $-1(2)$ & $-1(2)$ \\
\hline $\mathrm{C} 17$ & $19(2)$ & $18(2)$ & $21(2)$ & $-2(1)$ & $1(2)$ & $-2(2)$ \\
\hline C18 & $21(2)$ & $23(2)$ & $17(2)$ & $3(1)$ & $2(2)$ & $5(2)$ \\
\hline $\mathrm{C} 19$ & $21(2)$ & $17(2)$ & $11(2)$ & $3(1)$ & $-2(2)$ & $1(2)$ \\
\hline $\mathrm{C} 20$ & $19(2)$ & $23(2)$ & $16(2)$ & $0(1)$ & $2(2)$ & $-1(2)$ \\
\hline $\mathrm{C} 21$ & $21(2)$ & $18(2)$ & $16(2)$ & $4(1)$ & $0(2)$ & $5(2)$ \\
\hline $\mathrm{C} 22$ & $24(2)$ & $18(2)$ & $14(2)$ & $-2(1)$ & $1(2)$ & $1(2)$ \\
\hline $\mathrm{C} 23$ & $18(2)$ & $22(2)$ & $13(2)$ & $2(1)$ & $-2(1)$ & $0(2)$ \\
\hline $\mathrm{C} 24$ & $22(2)$ & $16(2)$ & $16(2)$ & $1(1)$ & $4(2)$ & $5(2)$ \\
\hline $\mathrm{C} 25$ & $15(2)$ & $22(2)$ & $12(2)$ & $0(1)$ & $2(1)$ & $-1(2)$ \\
\hline $\mathrm{C} 26$ & $19(2)$ & $20(2)$ & $19(2)$ & $-1(1)$ & $4(2)$ & $-4(2)$ \\
\hline
\end{tabular}




\begin{tabular}{|c|c|c|c|c|c|c|}
\hline $\mathrm{C} 27$ & $23(2)$ & $25(2)$ & $18(2)$ & 1(2) & $3(2)$ & $1(2)$ \\
\hline $\mathrm{C} 28$ & $23(2)$ & $23(2)$ & $19(2)$ & $4(2)$ & $4(2)$ & $1(2)$ \\
\hline $\mathrm{C} 29$ & $22(2)$ & $19(2)$ & $24(2)$ & $2(2)$ & $8(2)$ & $-1(2)$ \\
\hline $\mathrm{C} 30$ & $20(2)$ & $22(2)$ & $16(2)$ & $-1(1)$ & $5(2)$ & $-4(2)$ \\
\hline $\mathrm{C} 31$ & $28(2)$ & $16(2)$ & $24(2)$ & $0(2)$ & $2(2)$ & $-1(2)$ \\
\hline $\mathrm{C} 32$ & $38(2)$ & $31(2)$ & $36(2)$ & $6(2)$ & $-2(2)$ & $-13(2)$ \\
\hline $\mathrm{C} 33$ & $33(2)$ & $27(2)$ & $50(3)$ & $-17(2)$ & $-1(2)$ & $3(2)$ \\
\hline $\mathrm{C} 34$ & $33(2)$ & $22(2)$ & $26(2)$ & $-2(2)$ & $-3(2)$ & $-3(2)$ \\
\hline $\mathrm{C} 35$ & $20(2)$ & $21(2)$ & $13(2)$ & $2(1)$ & $0(2)$ & $1(2)$ \\
\hline $\mathrm{C} 36$ & $21(2)$ & $44(3)$ & $17(2)$ & $-1(2)$ & $-3(2)$ & $3(2)$ \\
\hline $\mathrm{C} 37$ & $26(2)$ & $24(2)$ & $28(2)$ & $6(2)$ & $-3(2)$ & $1(2)$ \\
\hline C38 & $20(2)$ & $29(2)$ & $26(2)$ & $5(2)$ & $2(2)$ & $-1(2)$ \\
\hline C39 & $27(2)$ & $24(2)$ & $14(2)$ & $1(1)$ & $2(2)$ & $-1(2)$ \\
\hline $\mathrm{C} 40$ & $27(2)$ & $31(2)$ & $18(2)$ & $5(2)$ & $4(2)$ & $2(2)$ \\
\hline $\mathrm{C} 41$ & $30(2)$ & $27(2)$ & $28(2)$ & $2(2)$ & $10(2)$ & $6(2)$ \\
\hline $\mathrm{C} 42$ & $45(3)$ & $35(2)$ & $16(2)$ & $-2(2)$ & $4(2)$ & $-3(2)$ \\
\hline $\mathrm{C} 43$ & $30(2)$ & $19(2)$ & $23(2)$ & $0(2)$ & $3(2)$ & $-5(2)$ \\
\hline $\mathrm{C} 44$ & $44(3)$ & $16(2)$ & $58(3)$ & $-4(2)$ & $20(2)$ & $-2(2)$ \\
\hline $\mathrm{C} 45$ & $48(3)$ & $27(2)$ & $31(2)$ & $8(2)$ & $6(2)$ & $-8(2)$ \\
\hline $\mathrm{C} 46$ & $36(2)$ & $28(2)$ & $29(2)$ & $-3(2)$ & $6(2)$ & $-11(2)$ \\
\hline $\mathrm{C} 47$ & $18(2)$ & $19(2)$ & $19(2)$ & $-2(1)$ & $1(2)$ & $-1(2)$ \\
\hline C48 & $27(2)$ & $25(2)$ & $38(2)$ & $-5(2)$ & $9(2)$ & $-7(2)$ \\
\hline $\mathrm{C} 49$ & $19(2)$ & $40(3)$ & $24(2)$ & $-4(2)$ & $-4(2)$ & $-1(2)$ \\
\hline C50 & $28(2)$ & $26(2)$ & $24(2)$ & $-1(2)$ & $7(2)$ & $-4(2)$ \\
\hline C51 & $21(2)$ & $23(2)$ & $23(2)$ & $-4(2)$ & $7(2)$ & $-2(2)$ \\
\hline C52 & $29(2)$ & $41(3)$ & $30(2)$ & $-9(2)$ & $7(2)$ & $1(2)$ \\
\hline C53 & $33(2)$ & $31(2)$ & $40(2)$ & $-9(2)$ & $17(2)$ & $0(2)$ \\
\hline C54 & $32(2)$ & $48(3)$ & $26(2)$ & $-4(2)$ & $11(2)$ & $-2(2)$ \\
\hline C55 & $42(3)$ & $28(2)$ & $18(2)$ & $-2(2)$ & $4(2)$ & $4(2)$ \\
\hline C56 & $43(5)$ & $78(7)$ & $20(4)$ & $8(4)$ & $-8(3)$ & $-21(5)$ \\
\hline C57 & $51(5)$ & $89(6)$ & $23(4)$ & $-2(4)$ & $9(3)$ & $-26(4)$ \\
\hline C58 & $149(9)$ & $65(5)$ & 11(3) & $-2(3)$ & $6(5)$ & $32(6)$ \\
\hline C55' & $42(3)$ & $28(2)$ & $18(2)$ & $-2(2)$ & $4(2)$ & $4(2)$ \\
\hline C56' & $42(7)$ & $80(9)$ & $21(6)$ & $-7(5)$ & $19(5)$ & $17(6)$ \\
\hline C57' & $51(5)$ & $89(6)$ & $23(4)$ & $-2(4)$ & $9(3)$ & $-26(4)$ \\
\hline C58' & $149(9)$ & $65(5)$ & 11(3) & $-2(3)$ & $6(5)$ & $32(6)$ \\
\hline
\end{tabular}




\begin{tabular}{lllllll} 
C59 & $34(2)$ & $21(2)$ & $26(2)$ & $1(2)$ & $9(2)$ & $3(2)$ \\
C60 & $94(4)$ & $26(2)$ & $29(2)$ & $1(2)$ & $20(3)$ & $16(2)$ \\
C61 & $34(2)$ & $27(2)$ & $61(3)$ & $0(2)$ & $21(2)$ & $1(2)$ \\
C62 & $37(2)$ & $18(2)$ & $40(2)$ & $-2(2)$ & $7(2)$ & $1(2)$ \\
C63 & $57(4)$ & $41(5)$ & $61(5)$ & $-4(6)$ & $17(4)$ & $4(4)$ \\
C64 & $43(6)$ & $43(6)$ & $65(7)$ & $15(5)$ & $11(5)$ & $8(5)$ \\
O1 & $57(4)$ & $41(5)$ & $61(5)$ & $-4(6)$ & $17(4)$ & $4(4)$ \\
C65 & $45(6)$ & $42(6)$ & $60(6)$ & $16(5)$ & $18(5)$ & $-3(5)$ \\
C66 & $57(4)$ & $41(5)$ & $61(5)$ & $-4(6)$ & $17(4)$ & $4(4)$ \\
& & & & & & \\
\hline
\end{tabular}


Table S29. Hydrogen coordinates $\left(\times 10^{4}\right)$ and isotropic displacement parameters $\left(\AA^{2} \times 10^{3}\right)$ for neijk39.

\begin{tabular}{|c|c|c|c|c|}
\hline & $\mathrm{x}$ & $\mathrm{y}$ & z & $\mathrm{U}(\mathrm{eq})$ \\
\hline $\mathrm{H} 2 \mathrm{~A}$ & 6740 & 2114 & 2338 & 25 \\
\hline $\mathrm{H} 3 \mathrm{~A}$ & 7343 & 2451 & 1707 & 28 \\
\hline $\mathrm{H} 4 \mathrm{~A}$ & 6339 & 2487 & 988 & 28 \\
\hline $\mathrm{H} 5 \mathrm{~A}$ & 4735 & 2227 & 902 & 25 \\
\hline H8A & 4199 & -83 & 2186 & 22 \\
\hline H10A & 6070 & -1960 & 2868 & 23 \\
\hline $\mathrm{H} 12 \mathrm{~A}$ & 6540 & 816 & 3072 & 20 \\
\hline H14A & 5234 & 1604 & 3439 & 23 \\
\hline H16A & 6306 & 4134 & 3883 & 25 \\
\hline H18A & 5722 & 3544 & 2549 & 25 \\
\hline $\mathrm{H} 20 \mathrm{~A}$ & 4301 & 501 & 961 & 23 \\
\hline $\mathrm{H} 22 \mathrm{~A}$ & 2233 & -1419 & 589 & 23 \\
\hline $\mathrm{H} 24 \mathrm{~A}$ & 1920 & 699 & 1418 & 22 \\
\hline $\mathrm{H} 26 \mathrm{~A}$ & 2934 & 1982 & 547 & 23 \\
\hline $\mathrm{H} 28 \mathrm{~A}$ & 1979 & 4633 & 320 & 26 \\
\hline $\mathrm{H} 30 \mathrm{~A}$ & 2869 & 3699 & 1598 & 23 \\
\hline $\mathrm{H} 32 \mathrm{~A}$ & 4575 & -2721 & 2858 & 55 \\
\hline H32B & 3681 & -3056 & 2480 & 55 \\
\hline $\mathrm{H} 32 \mathrm{C}$ & 3641 & -2094 & 2747 & 55 \\
\hline H33A & 5263 & -2281 & 1811 & 58 \\
\hline H33B & 4615 & -3146 & 1885 & 58 \\
\hline $\mathrm{H} 33 \mathrm{C}$ & 5532 & -2884 & 2267 & 58 \\
\hline H34A & 3790 & -1289 & 1661 & 43 \\
\hline H34B & 3177 & -1238 & 2035 & 43 \\
\hline $\mathrm{H} 34 \mathrm{C}$ & 3208 & -2188 & 1757 & 43 \\
\hline H36A & 6861 & -617 & 3925 & 43 \\
\hline H36B & 7321 & 319 & 3779 & 43 \\
\hline $\mathrm{H} 36 \mathrm{C}$ & 7982 & -507 & 4031 & 43 \\
\hline H37A & 7639 & -2149 & 3136 & 41 \\
\hline H37B & 7091 & -2156 & 3536 & 41 \\
\hline
\end{tabular}




\begin{tabular}{|c|c|c|c|c|}
\hline $\mathrm{H} 37 \mathrm{C}$ & 8198 & -1951 & 3646 & 41 \\
\hline H38A & 8231 & -675 & 2886 & 38 \\
\hline H38B & 8800 & -460 & 3392 & 38 \\
\hline H38C & 8080 & 301 & 3121 & 38 \\
\hline $\mathrm{H} 40 \mathrm{~A}$ & 6963 & 1756 & 4338 & 38 \\
\hline H40B & 6050 & 1143 & 4124 & 38 \\
\hline $\mathrm{H} 40 \mathrm{C}$ & 6273 & 1423 & 4648 & 38 \\
\hline H41A & 4675 & 2112 & 4545 & 41 \\
\hline H41B & 4431 & 1866 & 4017 & 41 \\
\hline $\mathrm{H} 41 \mathrm{C}$ & 4331 & 2922 & 4180 & 41 \\
\hline $\mathrm{H} 42 \mathrm{~A}$ & 6775 & 3434 & 4565 & 48 \\
\hline H42B & 6109 & 3066 & 4880 & 48 \\
\hline $\mathrm{H} 42 \mathrm{C}$ & 5742 & 3863 & 4511 & 48 \\
\hline $\mathrm{H} 44 \mathrm{~A}$ & 5168 & 5656 & 3163 & 57 \\
\hline H44B & 6057 & 6347 & 3265 & 57 \\
\hline $\mathrm{H} 44 \mathrm{C}$ & 5911 & 5608 & 3640 & 57 \\
\hline $\mathrm{H} 45 \mathrm{~A}$ & 5645 & 5194 & 2467 & 54 \\
\hline H45B & 6632 & 4688 & 2488 & 54 \\
\hline $\mathrm{H} 45 \mathrm{C}$ & 6599 & 5791 & 2584 & 54 \\
\hline $\mathrm{H} 46 \mathrm{~A}$ & 7530 & 4950 & 3690 & 47 \\
\hline H46B & 7688 & 5697 & 3320 & 47 \\
\hline $\mathrm{H} 46 \mathrm{C}$ & 7827 & 4600 & 3241 & 47 \\
\hline $\mathrm{H} 48 \mathrm{~A}$ & 1445 & -2036 & 1352 & 45 \\
\hline H48B & 1115 & -2180 & 817 & 45 \\
\hline $\mathrm{H} 48 \mathrm{C}$ & 348 & -2096 & 1116 & 45 \\
\hline H49A & 279 & 107 & 502 & 44 \\
\hline H49B & -373 & -767 & 559 & 44 \\
\hline $\mathrm{H} 49 \mathrm{C}$ & 477 & -903 & 313 & 44 \\
\hline $\mathrm{H} 50 \mathrm{~A}$ & 986 & -490 & 1687 & 38 \\
\hline H50B & -65 & -650 & 1397 & 38 \\
\hline $\mathrm{H} 50 \mathrm{C}$ & 475 & 308 & 1347 & 38 \\
\hline $\mathrm{H} 52 \mathrm{~A}$ & 5218 & -425 & 729 & 50 \\
\hline H52B & 5341 & -1403 & 490 & 50 \\
\hline $\mathrm{H} 52 \mathrm{C}$ & 5071 & -1393 & 972 & 50 \\
\hline H53A & 2986 & -2097 & 158 & 50 \\
\hline H53B & 3660 & -2420 & 627 & 50 \\
\hline
\end{tabular}




\begin{tabular}{|c|c|c|c|c|}
\hline $\mathrm{H} 53 \mathrm{C}$ & 4052 & -2391 & 176 & 50 \\
\hline H54A & 4017 & 119 & 48 & 52 \\
\hline H54B & 3140 & -542 & -164 & 52 \\
\hline $\mathrm{H} 54 \mathrm{C}$ & 4178 & -839 & -199 & 52 \\
\hline H56A & 1022 & 3162 & -691 & 74 \\
\hline H56B & 985 & 3934 & -312 & 74 \\
\hline H56C & 794 & 2853 & -221 & 74 \\
\hline H57A & 2638 & 3760 & -700 & 81 \\
\hline H57B & 3444 & 3768 & -243 & 81 \\
\hline H57C & 2584 & 4491 & -306 & 81 \\
\hline H58A & 2180 & 1690 & -105 & 115 \\
\hline H58B & 3152 & 2066 & -198 & 115 \\
\hline H58C & 2230 & 2029 & -601 & 115 \\
\hline H56D & 3760 & 3295 & -173 & 69 \\
\hline H56E & 3196 & 2649 & -576 & 69 \\
\hline H56F & 3509 & 2234 & -75 & 69 \\
\hline H57D & 1535 & 2255 & -639 & 81 \\
\hline H57E & 1009 & 2568 & -254 & 81 \\
\hline H57F & 1810 & 1779 & -149 & 81 \\
\hline H58D & 2532 & 4489 & -364 & 115 \\
\hline H58E & 1458 & 4248 & -371 & 115 \\
\hline H58F & 1929 & 3853 & -761 & 115 \\
\hline H60A & 3005 & 5281 & 1788 & 73 \\
\hline H60B & 1992 & 4852 & 1789 & 73 \\
\hline H60C & 2120 & 5963 & 1754 & 73 \\
\hline H61A & 695 & 4930 & 1075 & 59 \\
\hline H61B & 899 & 5433 & 636 & 59 \\
\hline H61C & 867 & 6040 & 1075 & 59 \\
\hline H62A & 3325 & 5963 & 1091 & 48 \\
\hline H62B & 2461 & 6666 & 1072 & 48 \\
\hline $\mathrm{H} 62 \mathrm{C}$ & 2504 & 6036 & 641 & 48 \\
\hline H63A & 5206 & 5185 & -1005 & 79 \\
\hline H63B & 5297 & 6004 & -637 & 79 \\
\hline $\mathrm{H} 63 \mathrm{C}$ & 4327 & 5442 & -795 & 79 \\
\hline H64A & 5073 & 4039 & -436 & 61 \\
\hline H64B & 6048 & 4604 & -276 & 61 \\
\hline
\end{tabular}




\begin{tabular}{lrrrr} 
H65A & 6084 & 4369 & 508 & 58 \\
H65B & 5139 & 3748 & 363 & 58 \\
H66A & 5262 & 4511 & 1103 & 79 \\
H66B & 4314 & 4798 & 748 & 79 \\
H66C & 5189 & 5506 & 853 & 79 \\
\hline
\end{tabular}


Table S30. Torsion angles $\left[{ }^{\circ}\right]$ for neijk39.

\begin{tabular}{|c|c|c|c|}
\hline C13-P1-C1-C2 & $40.6(3)$ & C31-C9-C10-C11 & $-179.7(3)$ \\
\hline C7-P1-C1-C2 & $-68.9(3)$ & C9-C10-C11-C12 & $1.5(5)$ \\
\hline Fe1-P1-C1-C2 & $166.7(3)$ & C9-C10-C11-C35 & $-177.8(3)$ \\
\hline C13-P1-C1-C6 & $-141.3(3)$ & C8-C7-C12-C11 & $-1.8(5)$ \\
\hline C7-P1-C1-C6 & 109.2(3) & $\mathrm{P} 1-\mathrm{C} 7-\mathrm{C} 12-\mathrm{C} 11$ & 179.3(3) \\
\hline Fe1-P1-C1-C6 & $-15.1(3)$ & $\mathrm{C} 10-\mathrm{C} 11-\mathrm{C} 12-\mathrm{C} 7$ & $0.1(5)$ \\
\hline C6-C1-C2-C3 & $0.9(5)$ & C35-C11-C12-C7 & $179.3(3)$ \\
\hline $\mathrm{P} 1-\mathrm{C} 1-\mathrm{C} 2-\mathrm{C} 3$ & $179.0(3)$ & C1-P1-C13-C14 & $-157.7(3)$ \\
\hline $\mathrm{C} 1-\mathrm{C} 2-\mathrm{C} 3-\mathrm{C} 4$ & $-0.8(5)$ & C7-P1-C13-C14 & $-51.6(3)$ \\
\hline $\mathrm{C} 2-\mathrm{C} 3-\mathrm{C} 4-\mathrm{C} 5$ & $0.7(5)$ & Fe1-P1-C13-C14 & $80.7(3)$ \\
\hline $\mathrm{C} 3-\mathrm{C} 4-\mathrm{C} 5-\mathrm{C} 6$ & $-0.8(5)$ & C1-P1-C13-C18 & $33.5(3)$ \\
\hline $\mathrm{C} 4-\mathrm{C} 5-\mathrm{C} 6-\mathrm{C} 1$ & $0.9(5)$ & C7-P1-C13-C18 & $139.6(3)$ \\
\hline $\mathrm{C} 4-\mathrm{C} 5-\mathrm{C} 6-\mathrm{P} 2$ & $176.9(3)$ & Fe1-P1-C13-C18 & $-88.1(3)$ \\
\hline $\mathrm{C} 2-\mathrm{C} 1-\mathrm{C} 6-\mathrm{C} 5$ & $-0.9(5)$ & $\mathrm{C} 18-\mathrm{C} 13-\mathrm{C} 14-\mathrm{C} 15$ & $-0.8(5)$ \\
\hline P1-C1-C6-C5 & $-179.2(2)$ & $\mathrm{P} 1-\mathrm{C} 13-\mathrm{C} 14-\mathrm{C} 15$ & $-169.7(3)$ \\
\hline C2-C1-C6-P2 & $-177.3(2)$ & $\mathrm{C} 13-\mathrm{C} 14-\mathrm{C} 15-\mathrm{C} 16$ & $-0.8(5)$ \\
\hline P1-C1-C6-P2 & $4.5(3)$ & C13-C14-C15-C39 & 178.1(3) \\
\hline C25-P2-C6-C5 & $-38.3(3)$ & $\mathrm{C} 14-\mathrm{C} 15-\mathrm{C} 16-\mathrm{C} 17$ & $2.1(5)$ \\
\hline C19-P2-C6-C5 & $67.6(3)$ & C39-C15-C16-C17 & $-176.8(3)$ \\
\hline Fe1-P2-C6-C5 & $-168.1(3)$ & $\mathrm{C} 15-\mathrm{C} 16-\mathrm{C} 17-\mathrm{C} 18$ & $-1.7(5)$ \\
\hline C25-P2-C6-C1 & $137.8(3)$ & $\mathrm{C} 15-\mathrm{C} 16-\mathrm{C} 17-\mathrm{C} 43$ & $179.7(3)$ \\
\hline C19-P2-C6-C1 & $-116.3(3)$ & $\mathrm{C} 16-\mathrm{C} 17-\mathrm{C} 18-\mathrm{C} 13$ & $0.1(5)$ \\
\hline Fe1-P2-C6-C1 & $8.1(3)$ & $\mathrm{C} 43-\mathrm{C} 17-\mathrm{C} 18-\mathrm{C} 13$ & $178.6(3)$ \\
\hline C1-P1-C7-C12 & $89.0(3)$ & C14-C13-C18-C17 & $1.1(5)$ \\
\hline C13-P1-C7-C12 & $-20.3(3)$ & $\mathrm{P} 1-\mathrm{C} 13-\mathrm{C} 18-\mathrm{C} 17$ & $169.9(3)$ \\
\hline Fe1-P1-C7-C12 & $-152.2(3)$ & $\mathrm{C} 25-\mathrm{P} 2-\mathrm{C} 19-\mathrm{C} 20$ & $92.3(3)$ \\
\hline C1-P1-C7-C8 & $-89.9(3)$ & C6-P2-C19-C20 & $-13.1(3)$ \\
\hline C13-P1-C7-C8 & $160.8(3)$ & Fe1-P2-C19-C20 & $-133.9(3)$ \\
\hline $\mathrm{Fe} 1-\mathrm{P} 1-\mathrm{C} 7-\mathrm{C} 8$ & $28.9(3)$ & $\mathrm{C} 25-\mathrm{P} 2-\mathrm{C} 19-\mathrm{C} 24$ & $-87.1(3)$ \\
\hline C12-C7-C8-C9 & $2.2(5)$ & C6-P2-C19-C24 & $167.5(2)$ \\
\hline P1-C7-C8-C9 & $-178.8(3)$ & Fe1-P2-C19-C24 & $46.6(3)$ \\
\hline C7-C8-C9-C10 & $-0.7(5)$ & C24-C19-C20-C21 & $0.8(5)$ \\
\hline C7-C8-C9-C31 & $177.8(3)$ & $\mathrm{P} 2-\mathrm{C} 19-\mathrm{C} 20-\mathrm{C} 21$ & $-178.6(2)$ \\
\hline C8-C9-C10-C11 & $-1.1(5)$ & C19-C20-C21-C22 & $0.6(5)$ \\
\hline
\end{tabular}




\begin{tabular}{|c|c|c|c|}
\hline C19-C20-C21-C51 & $177.9(3)$ & $\mathrm{C} 12-\mathrm{C} 11-\mathrm{C} 35-\mathrm{C} 37$ & $178.6(3)$ \\
\hline $\mathrm{C} 20-\mathrm{C} 21-\mathrm{C} 22-\mathrm{C} 23$ & $-1.5(5)$ & $\mathrm{C} 10-\mathrm{C} 11-\mathrm{C} 35-\mathrm{C} 38$ & $118.1(3)$ \\
\hline C51-C21-C22-C23 & $-178.8(3)$ & C12-C11-C35-C38 & $-61.1(4)$ \\
\hline $\mathrm{C} 21-\mathrm{C} 22-\mathrm{C} 23-\mathrm{C} 24$ & $0.9(5)$ & C10-C11-C35-C36 & $-122.8(3)$ \\
\hline $\mathrm{C} 21-\mathrm{C} 22-\mathrm{C} 23-\mathrm{C} 47$ & $179.8(3)$ & C12-C11-C35-C36 & $57.9(4)$ \\
\hline C22-C23-C24-C19 & $0.7(5)$ & C16-C15-C39-C42 & $-2.9(5)$ \\
\hline C47-C23-C24-C19 & $-178.2(3)$ & C14-C15-C39-C42 & $178.2(3)$ \\
\hline $\mathrm{C} 20-\mathrm{C} 19-\mathrm{C} 24-\mathrm{C} 23$ & $-1.5(5)$ & $\mathrm{C} 16-\mathrm{C} 15-\mathrm{C} 39-\mathrm{C} 40$ & $-123.6(4)$ \\
\hline $\mathrm{P} 2-\mathrm{C} 19-\mathrm{C} 24-\mathrm{C} 23$ & $178.0(3)$ & $\mathrm{C} 14-\mathrm{C} 15-\mathrm{C} 39-\mathrm{C} 40$ & $57.6(4)$ \\
\hline C19-P2-C25-C26 & $-25.6(3)$ & C16-C15-C39-C41 & $117.4(4)$ \\
\hline C6-P2-C25-C26 & $83.5(3)$ & C14-C15-C39-C41 & $-61.5(4)$ \\
\hline $\mathrm{Fe} 1-\mathrm{P} 2-\mathrm{C} 25-\mathrm{C} 26$ & $-155.1(2)$ & $\mathrm{C} 18-\mathrm{C} 17-\mathrm{C} 43-\mathrm{C} 45$ & $3.3(5)$ \\
\hline C19-P2-C25-C30 & 155.1(3) & $\mathrm{C} 16-\mathrm{C} 17-\mathrm{C} 43-\mathrm{C} 45$ & $-178.2(3)$ \\
\hline C6-P2-C25-C30 & $-95.7(3)$ & C18-C17-C43-C44 & $123.4(4)$ \\
\hline $\mathrm{Fe} 1-\mathrm{P} 2-\mathrm{C} 25-\mathrm{C} 30$ & $25.6(3)$ & C16-C17-C43-C44 & $-58.1(4)$ \\
\hline $\mathrm{C} 30-\mathrm{C} 25-\mathrm{C} 26-\mathrm{C} 27$ & $1.8(5)$ & $\mathrm{C} 18-\mathrm{C} 17-\mathrm{C} 43-\mathrm{C} 46$ & $-117.0(4)$ \\
\hline $\mathrm{P} 2-\mathrm{C} 25-\mathrm{C} 26-\mathrm{C} 27$ & $-177.5(3)$ & $\mathrm{C} 16-\mathrm{C} 17-\mathrm{C} 43-\mathrm{C} 46$ & $61.6(4)$ \\
\hline $\mathrm{C} 25-\mathrm{C} 26-\mathrm{C} 27-\mathrm{C} 28$ & $-1.8(5)$ & $\mathrm{C} 24-\mathrm{C} 23-\mathrm{C} 47-\mathrm{C} 50$ & $-15.2(4)$ \\
\hline $\mathrm{C} 25-\mathrm{C} 26-\mathrm{C} 27-\mathrm{C} 55$ & $-178.0(4)$ & $\mathrm{C} 22-\mathrm{C} 23-\mathrm{C} 47-\mathrm{C} 50$ & $165.9(3)$ \\
\hline $\mathrm{C} 25-\mathrm{C} 26-\mathrm{C} 27-\mathrm{C} 55^{\prime}$ & $173.7(5)$ & $\mathrm{C} 24-\mathrm{C} 23-\mathrm{C} 47-\mathrm{C} 48$ & $-135.7(3)$ \\
\hline $\mathrm{C} 26-\mathrm{C} 27-\mathrm{C} 28-\mathrm{C} 29$ & $0.9(5)$ & $\mathrm{C} 22-\mathrm{C} 23-\mathrm{C} 47-\mathrm{C} 48$ & $45.4(4)$ \\
\hline C55-C27-C28-C29 & $177.2(4)$ & C24-C23-C47-C49 & $104.8(4)$ \\
\hline $\mathrm{C} 55^{\prime}-\mathrm{C} 27-\mathrm{C} 28-\mathrm{C} 29$ & $-174.5(5)$ & $\mathrm{C} 22-\mathrm{C} 23-\mathrm{C} 47-\mathrm{C} 49$ & $-74.0(4)$ \\
\hline $\mathrm{C} 27-\mathrm{C} 28-\mathrm{C} 29-\mathrm{C} 30$ & $0.1(5)$ & C22-C21-C51-C52 & $-142.3(3)$ \\
\hline C27-C28-C29-C59 & $-178.1(3)$ & C20-C21-C51-C52 & $40.6(4)$ \\
\hline C28-C29-C30-C25 & $-0.1(5)$ & C22-C21-C51-C54 & $97.5(4)$ \\
\hline C59-C29-C30-C25 & $178.0(3)$ & C20-C21-C51-C54 & $-79.6(4)$ \\
\hline C26-C25-C30-C29 & $-0.8(5)$ & C22-C21-C51-C53 & $-21.7(5)$ \\
\hline P2-C25-C30-C29 & $178.5(3)$ & C20-C21-C51-C53 & $161.2(3)$ \\
\hline C8-C9-C31-C33 & $127.5(4)$ & C28-C27-C55-C57 & $61.0(7)$ \\
\hline C10-C9-C31-C33 & $-54.0(4)$ & C26-C27-C55-C57 & $-122.9(5)$ \\
\hline C8-C9-C31-C34 & $6.2(5)$ & C28-C27-C55-C58 & $-178.0(6)$ \\
\hline C10-C9-C31-C34 & $-175.2(3)$ & C26-C27-C55-C58 & $-1.9(8)$ \\
\hline C8-C9-C31-C32 & $-112.4(4)$ & C28-C27-C55-C56 & $-58.3(7)$ \\
\hline $\mathrm{C} 10-\mathrm{C} 9-\mathrm{C} 31-\mathrm{C} 32$ & $66.2(4)$ & C26-C27-C55-C56 & $117.8(5)$ \\
\hline C10-C11-C35-C37 & $-2.2(5)$ & C28-C27-C55'-C56' & $122.6(7)$ \\
\hline
\end{tabular}




$\begin{array}{lclc}\text { C26-C27-C55'-C56' } & -52.7(9) & \text { C30-C29-C59-C60 } & 2.3(5) \\ \text { C28-C27-C55'-C58' } & 1.0(9) & \text { C28-C29-C59-C60 } & -179.7(4) \\ \text { C26-C27-C55'-C58' } & -174.3(7) & \text { C30-C29-C59-C61 } & -118.1(4) \\ \text { C28-C27-C55'-C57' } & -117.6(7) & \text { C28-C29-C59-C61 } & 60.0(4) \\ \text { C26-C27-C55'-C57' } & 67.2(8) & \text { C63-C64-O1-C65 } & -176.8(14) \\ \text { C30-C29-C59-C62 } & 122.1(4) & \text { C64-O1-C65-C66 } & -179.5(13) \\ \text { C28-C29-C59-C62 } & -59.8(4) & & \end{array}$

Symmetry transformations used to generate equivalent atoms:

$\# 1-\mathrm{x}+1 / 2, \mathrm{y},-\mathrm{z}+1 / 2$ 